\title{
Productivity, Metabolism and Physiology of Free-Living Chemoautotrophic Epsilonproteobacteria
}

\author{
by \\ Jesse Christopher McNichol \\ B.Sc., First Class Honours with Distinction, Mount Allison University (2008) \\ Submitted to the Joint Program in Oceanography/Applied Ocean Science \& \\ Engineering \\ in partial fulfillment of the requirements for the degree of \\ Doctor of Philosophy \\ at the \\ MASSACHUSETTS INSTITUTE OF TECHNOLOGY \\ and the \\ WOODS HOLE OCEANOGRAPHIC INSTITUTION
}

September 2016

(C)2016 Jesse C. McNichol.

All rights reserved.

The author hereby grants to MIT and WHOI permission to reproduce and to distribute publicly paper and electronic copies of this thesis document in whole or in part in any medium now known or hereafter created.

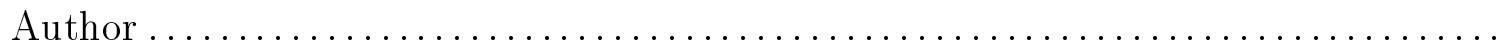

Joint Program in Oceanography/Applied Ocean Science \& Engineering Massachusetts Institute of Technology \& Woods Hole Oceanographic Institution August 15th, 2016

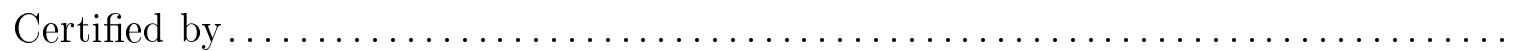

Dr. Stefan Sievert Associate Scientist with Tenure, Department of Biology Woods Hole Oceanographic Institution Thesis Supervisor

Accepted by .

Dr. Ann M. Tarrant

Chair, Joint Committee for Biological Oceanography Massachusetts Institute of Technology Woods Hole Oceanographic Institution 


\title{
Productivity, Metabolism and Physiology of Free-Living Chemoautotrophic Epsilonproteobacteria
}

\author{
by \\ Jesse Christopher McNichol
}

\author{
Submitted to the Joint Program in Oceanography/Applied Ocean Science \& Engineering \\ Massachusetts Institute of Technology \\ \& Woods Hole Oceanographic Institution \\ on August 15th, 2016, in partial fulfillment of the \\ requirements for the degree of \\ Doctor of Philosophy
}

\begin{abstract}
Chemoautotrophic ecosystems at deep-sea hydrothermal vents were discovered in 1977, but not until 1995 were free-living autotrophic Epsilonproteobacteria identified as important microbial community members. Because the deep-sea is food-starved, the autotrophic metabolism of hydrothermal vent Epsilonproteobacteria may be very important for deep-sea consumers. However, quantifying their metabolic activities in situ has remained difficult, and biochemical mechanisms underlying their autotrophic physiology are poorly described. To gain insight into environmental processes, an approach was developed for incubations of microbes at in situ pressure and temperature (25 $\mathrm{MPa}$, $24^{\circ} \mathrm{C}$ ) with various combinations of electron donors/acceptors $\left(\mathrm{H}_{2}, \mathrm{O}_{2}\right.$ and $\mathrm{NO}_{3}{ }^{-}$and $\left.{ }^{13} \mathrm{HCO}_{3}{ }^{-}\right)$ as a tracer to track carbon fixation. During short (18-24 h) incubations of low-temperature vent fluids from Crab Spa $\left(9^{\circ} \mathrm{N}\right.$ East Pacific Rise), the concentration of electron donors/acceptors and cell numbers were monitored to quantify microbial processes. Measured rates were generally higher than previous studies, and the stoichiometry of microbially-catalyzed redox reactions revealed new insights into sulfur and nitrogen cycling. Single-cell, taxonomically-resolved tracer incorporation showed Epsilonproteobacteria dominated carbon fixation, and their growth efficiency was calculated based on electron acceptor consumption. Using these data, in situ primary productivity, microbial standing stock, and average biomass residence time of the deep-sea vent subseafloor biosphere were estimated. Finally, the population structures of the most abundant genera Sulfurimonas and Thioreductor were shown to be strongly influenced by $\mathrm{pO}_{2}$ and temperature respectively, providing a mechanism for niche differentiation in situ. To gain insights into the core biochemical reactions underlying autotrophy in Epsilonprotebacteria, a theoretical metabolic model of Sulfurimonas denitrificans was developed. Validated iteratively by comparing in silico yields with data from chemostat experiments, the model generated hypotheses explaining critical, yet so far unresolved reactions supporting chemoautotrophy in Epsilonproteobacteria. For example, it provides insight into how energy is conserved during sulfur oxidation coupled to denitrification, how reverse electron transport produces ferredoxin for carbon fixation, and why aerobic growth yields are only slightly higher compared to denitrification. As a whole, this thesis provides important contributions towards understanding core mechanisms of chemoautrophy, as well as the in situ productivity, physiology and ecology of autotrophic Epsilonproteobacteria.
\end{abstract}

Thesis Supervisor: Dr. Stefan Sievert

Title: Associate Scientist with Tenure, Department of Biology

Woods Hole Oceanographic Institution 


\section{Acknowledgments}

I owe a great debt of gratitude to the kind and supportive presence of my advisor, Stefan Sievert. When I entered graduate school, I only had a vague understanding of the complex project I was to be entrusted with. It is a testament to Stefan's belief in me that he was able to give me the freedom and encouragement to make this project my own. I have also come to realize how important the conceptual groundwork Stefan laid was for my success. Stefan's understanding of the important and unresolved questions about hydrothermal vent ecosystems meant that my thesis project was both challenging and meaningful. Stefan has always given thoughtful feedback on all of my scholarly writing, and has encouraged me to take pride in my accomplishments. I feel very grateful for the responsibility, resources, and guidance I was given to make my thesis successful and am excited to continue working with Stefan in the future. Many thanks also to Stefan's family - Christel, Lukas and Helen - for always making me feel welcome at their home and in Cape Cod.

Another person key to my success at WHOI was Jeff Seewald. With Jeff's patience and can-do attitude, the IGT incubations became successful far beyond my expectations. When I expressed a desire to make oxygen measurements in hydrothermal fluids, Jeff found a way to make it happen. When there were challenges at sea, he faced them with a calm, optimistic and down-to-earth attitude. On land, we had many valuable discussions about writing papers and research where he brought a mature 'big picture' understanding of the hydrothermal vent environment to bear. I have learned much from Jeff that I will carry with me beyond WHOI and will always be grateful for his kindness towards me during my time in the joint program.

I also owe many thanks to Craig Taylor, whose assistance and encouragement with designing equipment for the IGT incubations was greatly appreciated. Receiving practical help, kind words and encouragement from an eminent "veteran" of deep-sea research like Craig has meant an enormous amount to me. I'll also never forget all the laughs on cruises from Craig's many hilarious stories and anecdotes.

While I was in Leipzig, Germany to analyze field samples, Niculina Musat was kind enough to take me under her wing and teach me the fundamentals of CARD-FISH. Under her careful tutelage, I was finally able to see the microbes that I was studying, which was an incredibly rewarding experience. She and others at UFZ Leipzig - Hryhoriy, Sabrina, Florin 
and Matthias, were all kind and helpful, and made me feel very welcome while in a foreign country.

Thanks are also due to members of my thesis committee: Costa Vetriani, Colleen Hansel, Roger Summons and Tanja Bosak. All of these experienced scientists took time from their busy schedules to give me constructive and encouraging feedback throughout the last three years of my degree. Thanks also to Roger and Mike Neubert for serving on my academic advisory committee and many thanks to Amy Apprill for her professionalism during both my proposal and thesis defense.

Work in the lab and in the field would not have been nearly as productive or fun without the guidance and encouragement from and the sense of humour of many friends and colleagues. Big thanks to François Thomas for being both a mentor and friend in the lab and at sea. François never complained once despite the insane amount of work and odd hours we kept to monitor the "little boys" in the IGTs. Whether in the field or lab, François is a calm and reassuring presence and has continued to give thoughtful advice to me even after leaving WHOI. Many thanks also go to Sean Sylva, a kind and encouraging gentleman who always lent a helping hand at sea or on shore no matter how demanding the request was from a troublesome "biologist" such as myself. Ying Zhang also deserves thanks for her guidance and introduction to genome-scale metabolic modeling, which has formed an important component of my thesis work. I am also grateful to Marc Mussmann and Stefan Dyksma for a productive collaboration and for the opportunity to learn from their expertise in CARD-FISH and marine microbiology in general. Thanks also to Fengping Wang and Xiang Xiao for hosting me in Shanghai during my academic exchange.

Other members of the Sievert lab, both past and present, have also made my time at WHOI a fantastic experience. Lara Gulmann has always offered me a helping hand with sequence analysis and lab work, and I am also grateful for the kindness her family has shown me. Special thanks go to Ben and Eric for not only attending my thesis defense presentation but also kindly offering me their heartfelt congratulations afterwards! Big ups also to Emelia Deforce for being a high-energy, nurturing presence throughout much of my time at WHOI. Camila Signori has always been a great friend and colleague during my time at WHOI, and has shared with me many of the joys and frustrations of research in the lab and at sea. Florian Götz has likewise been a great friend and colleague, who always shares what he has with others (including many delicious fish he caught himself) and has never hesitated to help 
me out in times of need. Thanks also to Sayaka Mino, who I came to respect as a researcher and friend during her short time at WHOI. Finally, thanks are due to Dali Smolsky for her help with isolation and cultivation during her time in the Sievert lab.

Life in the joint program was made so much richer by the friends I met while here. Many laughs and fun times were had with Peter Sullivan while living in Boston chez "quark quant". I couldn't have asked for a better rooommate in Alec Bogdanoff while living in Woods Hole, who went out of his way to make my time more comfortable and enjoyable. Thanks to Izi, Harriet and Julie for being great friends, running buddies and fellow board game fanatics. And to all my other friends, colleagues and teachers at WHOI and MIT, thank you for your kindness, friendship and support throughout these past 5 years.

And last but not least, I owe a different but no less important debt of gratitude to my family. I often think that my Nana \& Grandad as well as my Grandma \& Grandpa, who encouraged a love of nature in me at a young age, would have been proud to see me pursuing my curiosity. Even bigger and heartfelt thanks are due to my parents, who have always encouraged me to follow my interests and given me the support and love I needed to succeed. To my brothers, who always lent a sympathetic ear and made me laugh when I was stressed out during the past five years. To Karen, my partner in life - even though you weren't always here with me in person, your unwavering support and love was always felt and has given me the confidence and inspiration I needed to get to this point. I am very glad to have been welcomed into your family, and am grateful for their kind support and encouragement over the years. I couldn't be more excited to begin a new, lifelong adventure with you.

This research was funded by a grant of the Dimensions of Biodiversity program of the National Science Foundation to Stefan M. Sievert, Jeffrey S. Seewald, and Craig D. Taylor (NSF-OCE-1136727). Funding for Jesse McNichol was provided by doctoral fellowships from the National Sciences and Engineering Research Council of Canada (NSERC-PGSD3; PGSM-405117-2011, PGSD-439487-2013) and the National Aeronautics and Space Administration Earth Systems Science Fellowship (NESSF; PLANET14F-0075), an award from the Canadian Meteorological and Oceanographic Society, as well as funding from WHOI academic programs and the aforementioned National Science Foundation grant. 


\section{Contents}

1 Background and Rationale: $\quad 17$

1.1 Chemoautotrophy and Deep-Sea Hydrothermal Vents: . . . . . . . . . . . 17

1.2 The Discovery of Chemoautotrophic

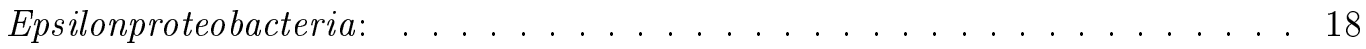

1.3 The Ecological Milieu of Vent Epsilonproteobacteria : . . . . . . . . . . 20

1.4 The Physiology of Autotrophic Epsilonproteobacteria . . . . . . . . . . . 22

1.5 Open Questions: . . . . . . . . . . . . . . . . . . . 27

1.6 Addressing These Questions Experimentally: . . . . . . . . . . . . . . 28

1.7 Linking Microbial Metabolism and Taxonomy in Mixed Communities: . . . . 29

1.8 Guiding Questions: . . . . . . . . . . . . . . . . . 30

1.9 Chapters 2 and 3 - Field Research on Natural Hydrothermal Vent Microbial

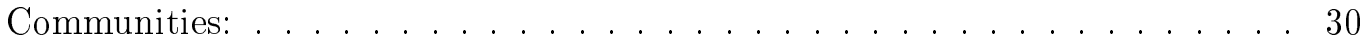

1.10 Chapter 4 - Theoretical Investigations into Chemoautotrophy in Sulfurimonas

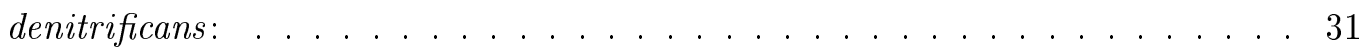

2 Assessing Microbial Processes in Deep-Sea Hydrothermal Systems by Incubation at In Situ Temperature and Pressure 33

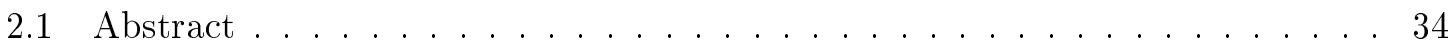

2.2 Introduction . . . . . . . . . . . . . . . . . . 35

2.3 Materials and Methods . . . . . . . . . . . . . . . . 37

$2.3 .1 \quad$ Field Site . . . . . . . . . . . . . . . . 37

2.3.2 Experimental Design and Methods for Incubations . . . . . . . . . . . 37

2.3.3 Analytical Methods: . . . . . . . . . . . . . . . . . . 42

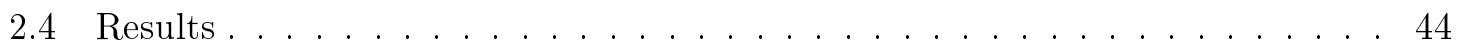


2.4.1 Crab Spa Microbial Community Composition and Fluid Chemistry: . . 44

2.4 .2 Incubation results: . . . . . . . . . . . . . . . . . . . 46

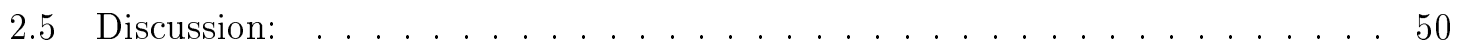

2.5.1 Chemosynthesis at $\mathrm{Crab} S \mathrm{Spa} \ldots \ldots \ldots \ldots$................... 50

2.5.2 Biogeochemistry of Sulfur and Nitrogen: . . . . . . . . . . . . 55

2.5.3 Rates of Chemosynthetic Processes at in situ Pressure and Temperature: 58

2.6 Summary: . . . . . . . . . . . . . . . . . . . . . 62

2.7 Acknowledgements: . . . . . . . . . . . . . . . . . 62

3 Physiologically Diverse Epsilonproteobacteria Dominate Subseafloor Autotrophy at a Deep-Sea Hydrothermal Vent 65

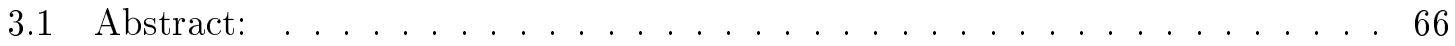

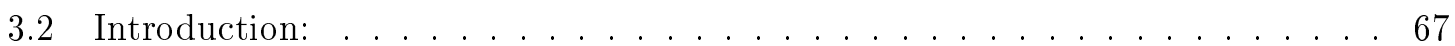

3.3 Materials and Methods: . . . . . . . . . . . . . . 67

3.3 .1 DNA Analyses: . . . . . . . . . . . . . . . . . . 68

3.3.2 CARD-FISH $/{ }^{13} \mathrm{C}$ incorporation: . . . . . . . . . . . . . . . 69

3.3.3 ${ }^{13} \mathrm{C}$ isotope incorporation into bulk biomass: . . . . . . . . . . . . 70

3.3.4 Chemosynthetic growth efficiency (CGE) determinations: . . . . . . 71

3.3.5 Extrapolations to natural environment: . . . . . . . . . . . 71

3.4 Results and Discussion: . . . . . . . . . . . . . . . . . . 73

3.5 Acknowledgements: . . . . . . . . . . . . . . . . 81

4 A Physiologically-Reconciled Model of an Epsilonproteobacterial Chemolithoau$\begin{array}{ll}\text { totroph } & 83\end{array}$

4.1 Abstract: . . . . . . . . . . . . . . . . . 84

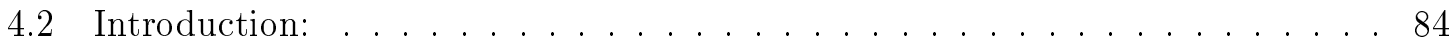

4.3 Materials and Methods: . . . . . . . . . . . . . . . . . . 86

4.3.1 Genome-scale metabolic modeling: . . . . . . . . . . . . 86

4.3.2 Chemostat cultivation and growth yield measurements: . . . . . . . . . 89

4.4 Results: . . . . . . . . . . . . . . . . . . . . 90

4.4.1 Chemostat cultivation: . . . . . . . . . . . . . . 90

4.4.2 Curation of ModelSEED-predicted reactions: . . . . . . . . . . . . 90

4.4 .3 Core chemoautotrophic enzymes: . . . . . . . . . . . . . . . . . 92 
4.4.4 Energy Conservation Efficiency of Enzymatic Complexes: . . . . . . . 96

4.5 Discussion: . . . . . . . . . . . . . . . . . . . . . . . . 99

4.5.1 Thiosulfate- and Hydrogen-Dependent Denitrification: . . . . . . . . . 100

4.5.2 Aerobic Respiration vs. Denitrification: . . . . . . . . . . . . . . 104

4.5.3 Reverse Electron Transport: . . . . . . . . . . . . . . . . . . . . 105

4.6 Conclusions: . . . . . . . . . . . . . . . . . . . . . . 108

4.7 Acknowledgements: . . . . . . . . . . . . . . . . . . . . 109

5 Conclusions and Future Work: 111

5.1 Hydrothermal Fluid Incubations: . . . . . . . . . . . . . . . . . . 111

5.2 Genome Scale Metabolic Modeling: . . . . . . . . . . . . . . . . . . . 115

5.3 Ongoing Research: . . . . . . . . . . . . . . . . . . 117

A Supplemental Material for Chapter 3 119

B Supplemental Material for Chapter 4 127 


\section{List of Figures}

1-1 Chemoautotrophy in deep-sea hydrothermal vent Epsilonproteobacteria . . . . 18

1-2 Conceptual diagram of fluid mixing at Crab Spa. . . . . . . . . . . . . 21

2-1 Isobaric gas-tight (IGT) sampler configurations used to amend Crab Spa hydrothermal fluids with inorganic substrates, monitor chemical consumption, and track microbial growth. . . . . . . . . . . . . . . . 39

2-2 Incubation results for 6 different treatment types. . . . . . . . . . . . . . . 49

2-3 Community redox balance for IGT incubations represented as a ratio. $\quad . \quad$. . 56

3-1 Bacterial community composition during incubations. . . . . . . . . . . . . 74

3-2 Relative estimations of primary productivity in incubations of hydrothermal vent fluids at in situ temperature and pressure determined by halogen in-situ hybridization secondary ion mass spectrometry (HISH-SIMS) . . . . . . . . 76

3-3 Fraction of electrons from electron donors used for carbon fixation (chemosynthetic growth efficiency). . . . . . . . . . . . . . . . 77

4-1 Hypothetical mechanisms of electron transport and energy conservation during denitrification in Sulfurimonas denitrificans with thiosulfate (A) or hydrogen (B) as an electron donor. . . . . . . . . . . . . . . . . 94

4-2 Hypothetical mechanism of reverse electron transport in Sulfurimonas deni-

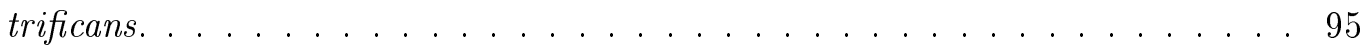

4-3 Hypothetical model of electron transport and energy conservation during aerobic respiration coupled to sulfur oxidation in Sulfurimonas denitrificans. . . . 100

A-1 Correlation between the proportion of cells hybridized to the Nautiliales-specific probe (NAUT921) and ammonium production. . . . . . . . . . . 120 
A-2 Thioreductor 97\% OTU composition across different treatment conditions. . . 121

A-3 Absolute carbon fixation rates across different treatment conditions derived by combustion of unfixed bulk microbial biomass. . . . . . . . . . . . . . . . . 122 


\section{List of Tables}

2.1 Summary of conditions during high pressure incubations using IGT fluid sam-

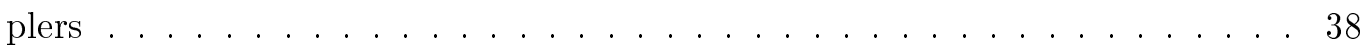

2.2 Measured and predicted concentrations of selected aqueous species at the

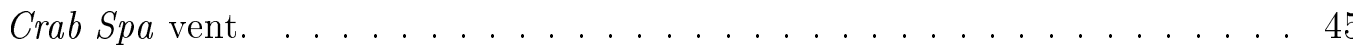

2.3 Percentage of DAPI-stained cells hybridizing to specific CARD-FISH probes in Crab Spa vent fluids and experimental amendments of Crab Spa fluids after 16-24 h of incubation. . . . . . . . . . . . . . . . 46

2.4 Summary of rates of change in chemical concentrations and cell densities. . . 51

2.5 Important predicted redox reactions in incubations of Crab Spa fluids that are most likely microbially catalyzed. . . . . . . . . . . . . . . . 54

4.1 Model summary statistics. . . . . . . . . . . . . . . . . . . . . . 87

4.2 Growth yields predicted from in silico metabolic modeling compared with growth yields during chemostat cultivation . . . . . . . . . . . . . 97

4.3 Hypothetical biochemical mechanisms and experimental tests. . . . . . . . . 103

A.1 Proportion of cells affiliated to different taxonomic groups under different incubation conditions as determined by hybridization of specific oligonucleotide probes and signal amplification with catalyzed reporter deposition fluorescence in-situ hybridization (CARD-FISH) . . . . . . . . . . . . . . 123

A.2 Probe sequences used in this study and predicted coverage based on testprobe (http://www.arb-silva.de/search/testprobe/). . . . . . . . . . . . . . 124

A.3 Correlations between initial chemical parameters and final Sulfurimonas OTU composition after incubation for $\approx 24 \mathrm{~h}$ at in situ temperature and pressure. . 125 
A.4 Correlations between abundance and $\mathrm{pO}_{2}$ for the 24 most abundant Sulfurimonas OTUs ( $\geq 2 \%$ of total) after incubation for $\approx 24 \mathrm{~h}$ at in situ temperature and pressure. . . . . . . . . . . . . . . . . . 126 


\section{Chapter 1}

\section{Background and Rationale:}

\subsection{Chemoautotrophy and Deep-Sea Hydrothermal Vents:}

By converting inorganic chemicals into living biomass, autotrophic organisms produce most of the organic matter on Earth. In addition to autotrophy fueled by solar energy (i.e. photosynthesis), organic matter can also be produced when chemosynthetic autotrophs use energy derived from exergonic redox reactions between inorganic chemicals to support net carbon fixation. Otherwise known as chemoautotrophs, their unique metabolism was first recognized by Sergei Winogradsky in the late $19^{\text {th }}$ century (Dworkin, 2012). In 1977, they unexpectedly returned to the spotlight when deep-sea hydrothermal vents were discovered (hereafter referred to as deep-sea vents) (Ballard, 1977; Corliss et al., 1979). At these seafloor hot springs, animal communities with densities unprecedented for the deep sea were found clustered around actively venting fluids. Since very little photosynthetically-derived organic carbon reaches these depths, it became clear that an alternative source of nutrition was needed to sustain the high concentrations of biomass. Immediately after their discovery, it was hypothesized that reduced inorganic chemical species contained in fluids in combination with oxidized species in seawater supported deep-sea vent chemosynthetic microorganisms and in turn the abundant animal communities (Corliss et al., 1979).

Organic matter produced by chemoautotrophs at deep-sea vents can feed animal communities in one of two ways. Firstly, symbiotic microbes can provide direct nutrition to host animals (e.g Felbeck, 1981). Alternatively, free-living microbial communities can be fed upon by deep-sea animals (Jannasch, 1983). High concentrations of microbial biomass in warm hydrothermal fluids (Karl et al., 1980) and growth of microbes incubated at mod- 


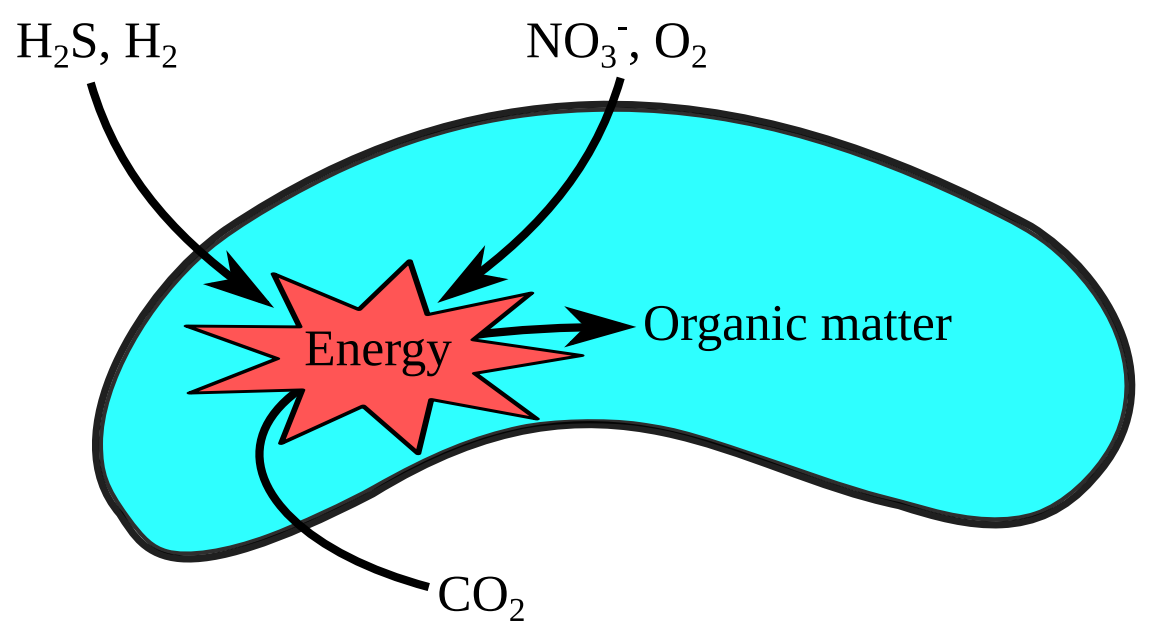

Figure 1-1: Chemoautotrophy in deep-sea hydrothermal vent Epsilonproteobacteria

erate temperatures suggest that a warm, subseafloor biosphere hosts substantial primary productivity and might be significant for carbon budgets in the deep sea (Tuttle et al., 1983; Jannasch and Mottl, 1985; Wirsen et al., 1986).

While activity was observed in the subseafloor biosphere and bacterial isolates from such environments had been characterized (Jannasch et al., 1985), the identity of microbes found in this habitat was initially unknown. However, biotechnological advances soon allowed SSU rRNA-based approaches and the associated phylogenetic framework of Woese and Fox (1977) to be applied to environmental samples (Pace, 1997). Equipped with new methods and this phylogenetic framework, scientists could finally identify the microbial inhabitants of hydrothermal vents without biases associated with cultivation.

\subsection{The Discovery of Chemoautotrophic Epsilonproteobacteria:}

Representatives of the metabolically diverse Proteobacteria (Stackebrandt et al., 1988) were quickly recognized as inhabitants of deep-sea vents. Gammaproteobacteria were first 
identified as symbionts of bivalves and siboglinid tube worms (Distel et al., 1988); this was consistent with the isolation of free-living chemosynthetic sulfur-oxidizing Gammaproteobacteria from deep-sea vents (Jannasch et al., 1985). However, another proteobacterial group - the Epsilonproteobacteria was later identified in both free-living and animal-associated microbial communities at deep-sea vents (Haddad et al., 1995; Polz and Cavanaugh, 1995). Although epsilonproteobacterial pathogens were known and an autotrophic representative had unknowingly been isolated (Timmer-Ten Hoor, 1975), they were not recognized as such until the advent of modern molecular methods (Muyzer et al., 1995).

In 2001, the successful isolation of deep-sea vent Epsilonproteobacteria was first reported (Campbell et al., 2001). A rough phylogenetic framework to delineate uncultivated groups was published the same year (Corre et al., 2001) and guided further isolation campaigns (Takai et al., 2003). These studies resulted in the description of the novel genera Sulfurimonas and Sulfurovum (Inagaki et al., 2003, 2004), now commonly identified in genomic surveys (e.g. Engel et al., 2004; Moussard et al., 2006; Huber et al., 2007) and known to actively fix $\mathrm{CO}_{2}$ under natural conditions (e.g. Grote et al., 2008; Ponsard et al., 2013). This work was soon followed by many other pure cultures (Nakagawa and Takai, 2008; Sievert and Vetriani, 2012, and references therein), and represented a major breakthrough as it permitted the genetic and physiological characterization of these organisms. More recently, meta-'omics' studies have shown that the same chemosynthetic pathways described in pure cultures are active in situ (Urich et al., 2014; Fortunato and Huber, 2016).

Epsilonproteobacterial autotrophs have now been found in sulfidic environments worldwide (Campbell et al., 2006). The deepest-branching (and presumably most ancient) lineages are all moderate thermophiles isolated from deep-sea vents (e.g. Zhang and Sievert, 2014), suggesting that Epsilonproteobacteria evolved in this habitat and only colonized other environments later (Campbell et al., 2006). Supporting this, even epsilonproteobacterial genera that associate with terrestrial animals, such as Campylobacter, Helicobacter and Wolinella, retain physiological characteristics reflective of a hydrothermal environment where they most likely evolved. These include a need for high $\mathrm{CO}_{2}$ (Al-Haideri et al., 2016), intolerance to high levels of $\mathrm{O}_{2}$ (Kendall et al., 2014), and the ability to use hydrogen as an electron donor (Wolin et al., 1961). Below, the underlying geochemistry of deep-sea vents and its effect on the growth conditions of the microbes inhabiting this habitat are discussed. 


\subsection{The Ecological Milieu of Vent Epsilonproteobacteria:}

Although fluids emitted at deep-sea hydrothermal vents originate from seawater, they become chemically transformed through their interaction with rock at high temperature and pressure. Tivey (2007) discusses this process and how the geological and physical setting affect the composition of a given site's hydrothermal end-member fluid. Such differences may be biologically-relevant, especially where concentrations of "energy-rich" reduced inorganics (i.e. $\mathrm{H}_{2}$ and $\mathrm{CH}_{4}$ ) differ. Despite these potential differences, we can also speak about general characteristics that differentiate hydrothermal fluids found at mid-ocean ridge (basalt-hosted) systems from surrounding seawater. These fluids are anoxic, rich in reduced gases and acidic. By contrast, seawater is cold, basic, oxic and contains almost no reduced compounds. Although markedly different fluids are produced at alkaline hydrothermal vents such as Lost City (Tivey, 2007), they were not investigated in this thesis, and fluids discussed below refer only to basalt-hosted mid-ocean ridge systems. In particular, the first two chapters of this thesis deal with low-temperature vent fluids from the $9^{\circ} 50^{\prime} \mathrm{N}$ East Pacific Rise (EPR) vent field.

Low-temperature hydrothermal fluids form as a result of mixing between end-member fluids and ambient seawater. The mixing of these chemically different fluids creates a redox disequilibrium, providing potential chemical energy for microbial communities below and above the seafloor. Because only two fluids are involved, the chemical environment for microorganisms varies along a mixing line of a two end-member model. Depending on the proportion of hydrothermal fluid, large variations in temperature and redox state exist for low-temperature fluids. Despite these biologically-relevant differences, some general characteristics of low-temperature fluids are similar across a wide range of mixing ratios and distinguish them from the surrounding deep sea.

A comprehensive dataset reported by Von Damm and Lilley (2004) discusses the composition of low-temperature hydrothermal fluids at $9^{\circ} 50^{\prime} \mathrm{N}$ EPR over many years of sampling and clearly demonstrates these similarities. Despite being composed primarily of seawater $(\approx 90 \%)$, the fluids sampled by Von Damm and Lilley (2004) are uniformly rich in sulfide, $\mathrm{CO}_{2}$, methane and hydrogen compared to seawater. As discussed by McCollom and Shock (1997), the chemical composition of fluids puts fundamental constraints on the possible extent of microbial metabolism. This is because sulfide (the primary electron donor) is typ- 


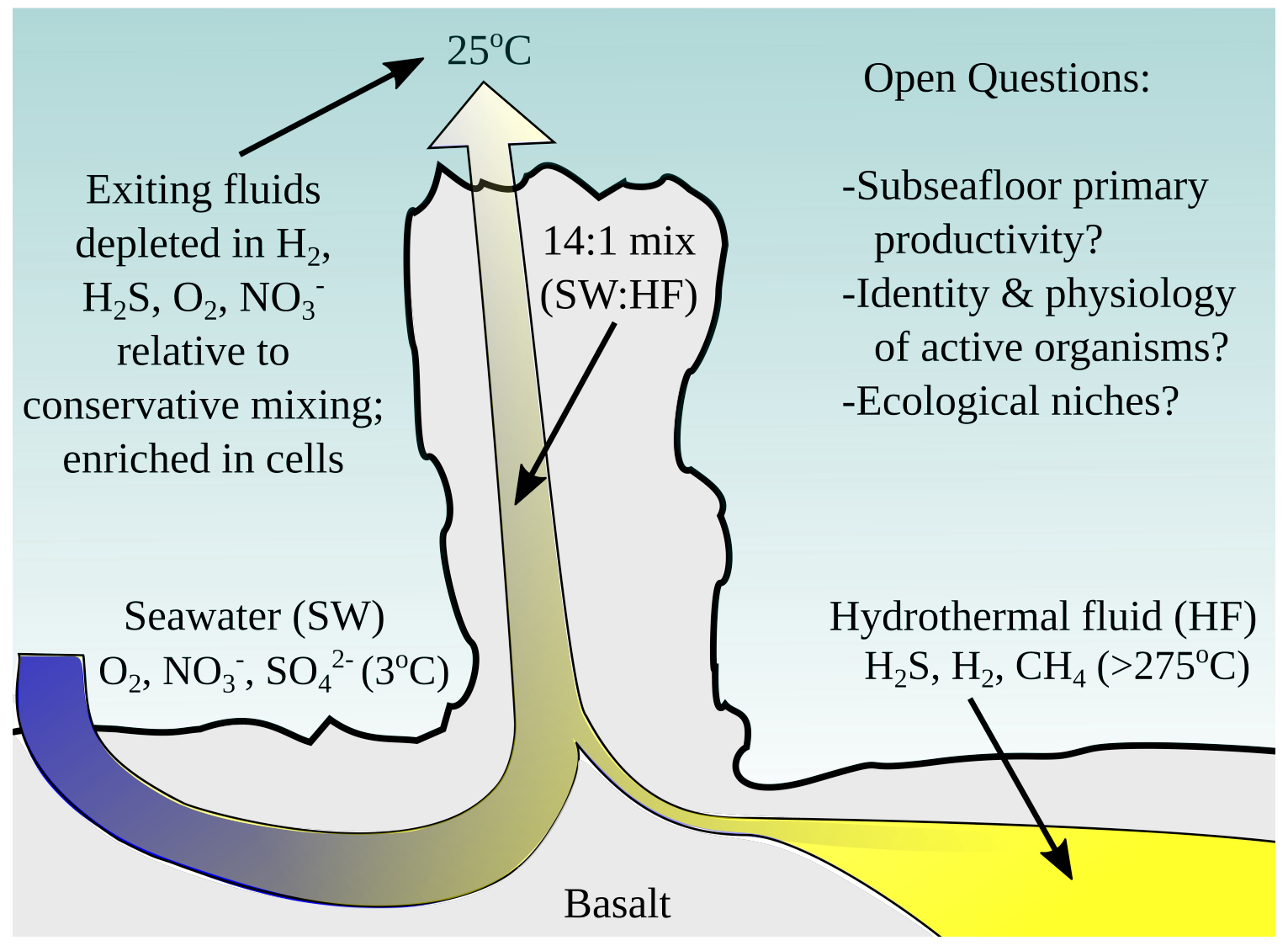

Figure 1-2: Conceptual diagram of fluid mixing at Crab Spa.

ically available in much larger quantities than oxygen derived from seawater (the primary electron acceptor). Therefore, subseafloor organisms are expected to be limited by electron acceptors except when the ratio of seawater to vent fluid is very high (McCollom and Shock, 1997). This remains true even when alternative electron acceptors such as nitrate are present (see Chapter 2). Finally, although hydrogen is much less abundant than sulfide in the subseafloor at $9^{\circ} 50^{\prime} \mathrm{N}$ EPR, it is apparently depleted by microbial metabolism based on end-member mixing models (Von Damm and Lilley, 2004, also see Chapter 2). These observations, combined with the bioenergetic rationale presented in Chapter 4, suggest that hydrogen is an important electron donor for subseafloor communities.

The nature of mixing also affects the environment that subseafloor organisms experience. For example, some low-temperature hydrothermal fluids are the result of very shallow mixing, evidenced by large temperature gradients. At such a site, microbes will be exposed to steep gradients of temperature and redox state on a small spatial scale ( $\mathrm{mm}$ to $\mathrm{cm})$. This can affect microbial population structures, with more thermophilic representatives found in 
such environments (Meier et al., 2016). In contrast, the Crab Spa vent site discussed in Chapters 2 and 3 appears to be the result of a deep and relatively constant mixing process, as evidenced by similar temperature and chemical composition since measurements began in 2007 (Sievert, Seewald, Le Bris and Luther, unpublished; see Fig 1-2). At Crab Spa, bacteria related to mesophilic Epsilonproteobacteria are dominant, suggesting the existence of a large subseafloor habitat with temperatures that are not significantly higher than venting fluids $\left(\approx 24^{\circ} \mathrm{C}\right)$. Together, these data suggest that conditions at Crab Spa are relatively constant compared to environments characterized by shallow mixing. From a practical standpoint, this made it possible to collect samples repeatedly from the same study site that were representative of the subseafloor community, in turn permitting the biological replication for experiments discussed below and in Chapters 2 and 3.

\subsection{The Physiology of Autotrophic Epsilonproteobacteria:}

As discussed above, many sulfidic habitats host large populations of free-living autotrophic Epsilonproteobacteria (Engel et al., 2004; Nakagawa et al., 2005b; Moussard et al., 2006). By contrast, autotrophic Gammaproteobacteria tend to inhabit less sulfidic niches where oxygen concentrations are higher (Macalady et al., 2008; Yamamoto and Takai, 2011; Hügler and Sievert, 2011). Ultimately, specific enzymes and pathways optimally adapted to the chemical environment likely underlie this observed niche differentiation. In recent years, genomic information and physiological characterization of isolates has revealed interesting clues into what allows Epsilonproteobacteria to thrive in their specific environmental niche. In the subsequent paragraphs, I consider how core pathways of energy generation and carbon fixation are phylogenetically distributed and related to environmental conditions.

To date, all characterized Epsilonproteobacteria are obligate respiratory organisms. Although capable in some cases of respiring fumarate, which has been considered by some as fermentation (Finster et al., 1997), this process is still catalyzed by a transmembrane protein and linked to quinol oxidation (Kröger et al., 2002). Therefore, fumarate reduction is essentially indistinguishable from other respiratory pathways found in Epsilonproteobacteria. For some Epsilonproteobacteria, electron acceptors are very diverse; Sulfurospirillum multivorans uses up to 13 distinct compounds as electron acceptors including chlorinated organics (Goris et al., 2014). Thus far, vent organisms have been shown to be less versatile. 
With the exception of Nautilia nitratireducens that can use selenate (Perez-Rodriguez et al., 2010), all other isolates can only use nitrate, oxygen and sulfur (in various intermediate redox states) as electron acceptors (Sievert and Vetriani, 2012).

For nitrate-respiring Epsilonproteobacteria, both denitrification to $\mathrm{N}_{2}$ and dissimilatory nitrate reduction to ammonium have been reported. Despite differences in the end product, the initial step $-\mathrm{NO}_{3}{ }^{-}$reduction to $\mathrm{NO}_{2}{ }^{-}$- is catalyzed by the periplasmic nitrate reductase (Nap), an enzyme complex that is evolutionarily conserved in both pathways (Vetriani et al., 2014). Although this complex conserves no energy because of its periplasmic location (Simon et al., 2008), its presence might permit organisms to more effectively compete in nitrate-limited environments (Potter et al., 1999). As discussed above, typical vent fluid chemistry ensures electron acceptor limitation; therefore, this high-affinity nitrate respiration complex is likely an important factor underlying the success of Epsilonproteobacteria in vent environments (Vetriani et al., 2014).

Electron donors for Epsilonproteobacteria can be either organic or inorganic compounds. Many heterotrophic isolates are known, including the well-studied pathogens Helicobacter and Campylobacter (Campbell et al., 2006). However, most vent isolates to date appear to be obligate autotrophs, with the exception of the thermophilic genus Nautilia. For N. profundicola and N. lithotrophica, formate is used as an electron donor, N. nitratireducens can use complex organics (Sievert and Vetriani, 2012, and references therein), and natural populations of Nautilia can assimilate acetate (Winkel et al., 2014). Mesophilic, heterotrophic Epsilonproteobacteria have not yet been isolated from vent systems. The reason for this remains unclear, but could be a cultivation bias as isolation media commonly used (Campbell et al., 2001; Takai et al., 2003) are selective for chemoautotrophs. However, genomic evidence from in situ populations of Epsilonproteobacteria thus far suggests that organic carbon utilization is restricted to small organics (Stokke et al., 2015). Furthermore, the fact that acetate amendments of natural fluids enrich for Epsilonproteobacteria (Nautilia) at $50^{\circ} \mathrm{C}$, but heterotrophic Gammaproteobacteria at $37^{\circ} \mathrm{C}$ suggests a limited heterotrophic potential within natural populations of mesophilic Epsilonproteobacteria at deep-sea hydrothermal vents (Winkel et al., 2014).

Despite different strategies for energy generation, all autotrophic Epsilonproteobacteria are united by a common carbon fixation pathway. Environmental gene surveys suggest the reductive tricarboxylic acid (rTCA) cycle is used by Epsilonproteobacteria 
at deep-sea vents (Campbell and Cary, 2004), and has since been biochemically confirmed in pure cultures (Hügler et al., 2005; Takai et al., 2005). Compared to the Calvin cycle, this pathway uses fewer ATP and so requires a smaller energy investment per $\mathrm{CO}_{2}$ fixed. However, two essential rTCA enzymes depend on reduced ferredoxin as an electron donor. As a result, iron-sulfur clusters associated with these proteins are exposed to the bulk solution of the cytoplasm in order to interact with ferredoxin as an electron donor. Since oxygen can directly inactivate such clusters (Imlay, 2006), it renders the entire pathway sensitive to oxygen and may underlie the oxygen-sensitive phenotype that distinguishes Epsilonproteobacteria from related chemoautotrophs (Yamamoto and Takai, 2011). Interestingly, despite the potential disadvantage to these organisms, these oxygen-sensitive enzymes appear evolutionarily conserved even in heterotrophic lineages where they operate in the oxidative direction (Tomb et al., 1997; Weerakoon and Olson, 2008).

Another important and poorly understood phenomenon is the capnophilic $\left(\mathrm{CO}_{2}\right.$-loving $)$ nature of Epsilonproteobacteria. As mentioned above, heterotrophic Campylobacter strains require high levels of $\mathrm{CO}_{2}(\approx 10 \% \mathrm{v} / \mathrm{v}$ in headspace gas $)$, which may be related to the activity of carbonic anhydrases that convert $\mathrm{CO}_{2}$ to bicarbonate for carboxylation reactions (Al-Haideri et al., 2016). Previous studies have shown that oxygen tolerance may be enhanced when $\mathrm{CO}_{2}$ above atmospheric levels is provided (Bolton and Coates, 1983). However, similar improvements in oxygen tolerance have been observed with humid air (Fraser et al., 1992), so it is not clear whether $\mathrm{CO}_{2}$ is responsible for this behavior. Although the precise physiological mechanisms underlying this capnophilic phenotype are not yet known, high $\mathrm{CO}_{2}$ concentrations are typical of the deep-sea vent subseafloor environment and have proven essential for the isolation of vent organisms (Campbell et al., 2001; Takai et al., 2003). This $\mathrm{CO}_{2}$-requiring phenotype is in stark contrast to the deep-sea vent gammaproteobacterium Thiomicrospira crunogena, which can tolerate low environmental $\mathrm{CO}_{2}$ concentrations by using a carbon-concentrating mechanism (e.g. Dobrinski et al., 2010).

Despite these general similarities, some essential metabolic enzymes vary across broad phylogenetic groupings of Epsilonproteobacteria. Although not officially recognized taxonomically, for the purposes of this section I separate autotrophic Epsilonproteobacteria into two broad groupings. These are the Nautiliales (Miroshnichenko et al., 2004) and the Campylobacterales (Garrity et al., 2015). Within the lineage Epsilonproteobacteria, the Nautiliales can be thought of as deep-branching, typically thermophilic organisms, whereas the 
Campylobacterales are shallower-branching mesophiles. Here, I define the order Nautiliales as in the SILVA taxonomy of Quast et al. (2013), i.e., including the genera Caminibacter, Cetia, Lebetimonas, Nautilia, Nitratifractor, Nitratiruptor and Thioreductor (Sievert and Vetriani, 2012; Grosche et al., 2015). For unknown reasons, Hydrogenimonas (Takai et al., 2004) appears as a separate group in the SILVA taxonomy, but here I treat it as part of the Nautiliales. All isolates belonging to the Nautiliales are strict anaerobes or microaerophiles also capable of respiring nitrate and sulfur. $\mathrm{H}_{2}$ is normally required as an electron donor, although formate and in some cases more complex organic matter can serve this role. In general, these organisms are thermophilic with temperature optima for growth $>40^{\circ} \mathrm{C}$, although Thioreductor (Nakagawa et al., 2005a) and Nitratifractor (Nakagawa et al., 2005c) are notable exceptions.

The autotrophic Campylobacterales include the genera Arcobacter, Sulfurovum, Sulfuricurvum, Sulfurimonas and Thiovulum. In general terms, their temperature optima are $\leq$ $33^{\circ} \mathrm{C}$, though higher temperatures can be tolerated in some cases (up to $40^{\circ} \mathrm{C}$ for Sulfurimonas autotrophica). While an Arcobacter has also been enriched from coastal sediments (Wirsen et al., 2002), no autotrophic deep-sea vent isolate has yet been brought into pure culture; the same is true for Thiovulum, found at sulfide-oxygen interfaces in sediments (Wirsen and Jannasch, 1978). In contrast to the Nautiliales, nearly all Campylobacterales isolates reported thus far have the potential to use oxygen, and in some cases tolerate levels approaching atmospheric concentrations (Inagaki et al., 2003; Takai et al., 2006). Even for those reported to be strict anaerobes, genome sequences contain a $\mathrm{cbb}_{3}$-type high-affinity cytochrome c oxidase (Grote et al., 2012; Cai et al., 2014), suggesting the ability to use oxygen as an electron acceptor is typical of autotrophic Campylobacterales. The use of nitrate and sulfur as electron acceptors is also common in this lineage but obligate aerobes do exist (Inagaki et al., 2003). Some Campylobacterales strains are reminiscent of the Nautiliales in terms of an obligate requirement for hydrogen (Mino et al., 2014), others are facultative hydrogen oxidizers (Takai et al., 2006), and yet others thrive with only reduced sulfur as an electron donor (Inagaki et al., 2003).

Significant differences also exist between these groups in terms of core energy-conserving protein complexes. In genomes from the deepest-branching Nautiliales such as Nautilia, Caminibacter and Lebetimonas (Campbell et al., 2009; Giovannelli et al., 2011; Meyer and Huber, 2014), no homologs of complex III are found. This energy-conserving complex trans- 
fers electrons from the membrane quinol pool to soluble cytochrome c, which is subsequently oxidized by complexes involved in aerobic respiration (e.g. cytochrome c oxidases) and denitrification (e.g. nitrogen oxide reductases other than nitrate reductases). Consistent with the absence of complex III, these deeper-branching organisms belonging to the Nautiliales are apparently incapable of reduction of nitrate to $\mathrm{N}_{2}$. Instead, they rely on a novel pathway for dissimilatory nitrate reduction to ammonium (DNRA) (Hanson et al., 2013). This suggests that autotrophic members of the Nautiliales have short, two-component respiratory chains that conserve energy via redox loops (Simon et al., 2008). The exceptions to this rule are Hydrogenimonas, Nitratifractor and Nitratiruptor, which are similar to the Campylobacterales in that they possess complex III, and can therefore carry out three-component pathways that require soluble cytochrome c.

In addition to complex III, the architecture of complex I (NADH:ubiquinone oxidoreductase; Nuo) varies markedly between these two lineages. In the aforementioned deepbranching Nautiliales genomes, complex I is consistently missing the NuoEFG subunits. Since these are the subunits that interact with electron donors in the canonical enzyme (Nicholls and Ferguson, 2013), the function of this truncated enzyme is unknown. For Campylobacterales genomes, they typically possess NuoEFG subunits in at least one copy of the Nuo complex. However, the NuoEF subunits are non-homologous to canonical complex I and may interact with ferredoxin/flavodoxin instead of NADH (Weerakoon and Olson, 2008). Another important feature in Nautiliales genomes is the presence of diverse hydrogenases (Meyer and Huber, 2014). The presence of so-called energy-converting hydrogenases (not yet found in any of the Campylobacterales) may be of particular importance to their autotrophic lifestyle since this complex is thought to produce ferredoxin during hydrogen oxidation at the expense of the proton motive force (Hedderich, 2004).

These data merely scratch the surface of metabolic pathways in autotrophic Epsilonproteobacteria; the precise biochemical mechanisms and physiological significance remain largely unexplored. Nonetheless, these studies have revealed that some central metabolic pathways are shared, possibly reflecting the warm, sulfide- and hydrogen-rich fluids in which they evolved. On the other hand, major differences between deep and shallow-branching lineages may be related to the evolution and diversification of Epsilonproteobacteria into more oxic environments (Campbell et al., 2006). 


\subsection{Open Questions:}

At this point, it is clear that Epsilonproteobacteria are dominant members of the microbial communities in habitats such as the subseafloor biosphere at deep-sea vents, and a general outline of their metabolism is known. However, three key uncertainties remain.

Firstly, a rigorous quantification of primary productivity at deep-sea vents is lacking, which is particularly true for the subseafloor biosphere. Without such an estimate, it is difficult to constrain the cycling of sulfur, hydrogen, nitrogen and oxygen used in chemosynthetic reactions (Sievert and Vetriani, 2012). Although studies have measured rates of carbon fixation in subseafloor-derived fluids (Tuttle et al., 1983; Wirsen et al., 1986; Perner et al., 2013), using these data to estimate in situ productivity relies on the assumption that experimentally-measured rates are reflective of rates in natural populations. However, carbon fixation rates in sulfidic environments can be underestimated during incubations by not measuring rates on a short enough timescale (Mandernack and Tebo, 1999), or possibly overestimated if chemical concentrations in incubations are higher than in situ. Therefore, directly applying rates to estimate primary productivity into the natural environment may not be a realistic approach.

Secondly, factors that control the composition of natural communities of Epsilonproteobacteria remain mostly unexplored. While temperature clearly differentiates deeperbranching Epsilonproteobacteria from shallow-branching strains as discussed above, the fact that putatively mesophilic genera co-exist at the same temperature in natural samples (Sievert, unpublished) suggests that other factors may play a role. Even within genera, there is considerable diversity in sequence composition (Huber et al., 2007), suggesting a variety

of niches likely exist in the natural environment. What factors define these niches? Does taxonomy correspond to physiology such that different lineages are optimally adapted to particular niches?

Finally, the fundamental physiology of autotrophic Epsilonproteobacteria is poorly understood. For example, sulfur-oxidizing chemoautotrophs need to produce cellular reductant for carbon fixation via reverse electron transport. In organisms using the Calvin cycle, the reversal of NADH:ubiquinone oxidoreductase (complex I) can fulfill this role. However, organisms that use the rTCA cycle require a source of reduced ferredoxin in addition to NADH. Mechanisms have yet to be identified to account for the generation of reduced ferredoxin 
in sulfur-oxidizing Epsilonproteobacteria (Marshall et al., 2012) though energy-converting hydrogenases could fulfill this role in hydrogen-dependent thermophiles.

\subsection{Addressing These Questions Experimentally:}

The first two questions relate to the environmental role of chemoautotrophic Epsilonproteobacteria. To make realistic inferences, it is therefore essential that experimental observations be made under conditions that simulate in situ conditions as close as possible. We can consider two general approaches to investigate microbial processes in the natural environment:

\section{Direct measurements in situ}

2. Incubation experiments with natural communities

The direct approach is minimally biased, as in situ measurements are not expected to greatly disrupt natural systems. Where depletions of chemicals in the natural environment are correlated with the presence of a particular microbial community, a circumstantial link can be drawn between these communities and the observed processes. However, such correlations are not definitive and cannot be used to directly infer rates of microbially-catalyzed processes.

The second approach - incubating microbial communities under simulated in situ conditions has a number of advantages. Cell abundances and rates of microbially-catalyzed redox reactions can be measured at high temporal resolution, and it is easier to experimentally address which organisms are active. In addition, incubations can investigate processes that occur in the natural environment, but are undetectable in situ due to the complete exhaustion of reactants by microbial metabolism.

However, the incubation-based approach has many challenges relevant for deep-sea vent research. Firstly, conditions must be maintained as close to in situ as possible to avoid biases; therefore, either incubations in the natural environment or a pressure-retaining sampler should be used. Secondly, since incubations occur in a closed system removed from the natural environment, extrapolations of measurements to the natural environment may introduce uncertainty. Therefore, careful experimental design and thorough data interpretation are necessary to ensure meaningful results. 


\subsection{Linking Microbial Metabolism and Taxonomy in Mixed Communities:}

In addition to appropriate experimental design, an equally important consideration is how to identify active microbes and quantify their metabolism. Although relatively straightforward in pure cultures, this is a challenging task when dealing with mixed communities. It is now trivial to amplify and sequence nucleic acids identified from the natural environment, but such sequences may derive from dead/inactive microbes and should be interpreted with caution (Klein, 2015). More flexible protocols that isolate only cellular DNA/RNA (Lever et al., 2015) or techniques to separate pools of nucleic acids that have incorporated a stableisotope tracer (Whiteley et al., 2006; Fortunato and Huber, 2016) can minimize this bias and provisionally identify active organisms.

However, techniques based solely on nucleic acid sequences are blind to many ecologically relevant parameters. Firstly, processes in the natural environment are rarely due to one organism, and measuring activity in the context of spatial relationships can be critically important (e.g. Orphan et al., 2001). Secondly, visualizing morphology and activity simultaneously can be essential for identifying the role of novel organisms in ecosystems (Marzocchi et al., 2014). Finally, correlating taxonomically-resolved measurements of activity with cell size and abundance can reveal how rare organisms contribute to overall ecological function (Musat et al., 2008).

None of these important questions can be addressed without a way to visually identify phylogenetically distinct cells in mixed populations. Since morphology is not an evolutionarily conserved trait, the hybridization of short nucleotides containing a label (probe) that binds to a taxonomically informative molecule such as rRNA inside the cell has become an essential tool for microbial ecologists to identify organisms in their natural milieu (DeLong et al., 1989; Amann et al., 1990). This process relies on rRNA databases, and is another important outcome of the seminal work of Woese and Fox (1977). The most commonly used label is a fluorescent dye, giving rise to the term fluorescence in-situ hybridization or "FISH". Since the advent of this molecular FISHing, specialized applications have been developed to amplify signals, target functional genes, and to use other labels to allow detection by other means, such as nanoscale secondary ion mass spectrometry (NanoSIMS; Pernthaler et al., 2002; Moraru et al., 2010; Musat et al., 2008; Amann and Moraru, 2012). 
If an appropriately specific probe is available, the next step is to correlate taxonomic identity with activity. To accomplish this, stable isotope or radio-labeled compounds of interest are added to incubations where they are incorporated by active microbes into cell material. Subsequent single-cell visualization may occur with radioactive (Ouverney and Fuhrman, 1999) or stable isotope-specific techniques such as Raman-FISH or NanoSIMS (Huang et al., 2007; Musat et al., 2008). These are the only techniques that can currently make measurements of activity at a single-cell, taxonomically-resolved level. NanoSIMS in particular is unparalleled in its spatial resolution, sensitivity, and quantitative nature of the analysis (Musat et al., 2012) and has been used to confirm predicted metabolic interactions in the natural environment (Thompson et al., 2012).

\subsection{Guiding Questions:}

Three inter-related questions that arise from the above discussion can be summarized as follows:

1. What is the biogeochemical significance of natural populations of autotrophic Epsilonproteobacteria at deep-sea hydrothermal vents?

2. What factors influence their ecology?

3. What putative biochemical mechanisms support their autotrophic growth?

\subsection{Chapters 2 and 3 - Field Research on Natural Hydrother- mal Vent Microbial Communities:}

The first two guiding questions are addressed in a series of experiments that have been separated into two chapters. Chapter 2 of this thesis discusses novel methods for short-term incubations of microbial communities under deep-sea vent temperature and pressure conditions. Aside from realistic incubation conditions, several elements of the experimental design distinguish our study from those previously reported. As observed by others (Von Damm and Lilley, 2004; Wankel et al., 2011; Butterfield et al., 2004) and ourselves, many chemicals are depleted in natural low-temperature hydrothermal vent fluids. This suggests, but does not prove that microbes in the natural environment are responsible for such activity. 
In our experiments, microbial consumption of substrates was confirmed and quantified by providing selected chemicals as amendments. In addition, chemical concentrations and cell abundances were measured in short $(\approx 24 \mathrm{~h}$ ) time-course incubations (sampling every $\approx 6$ h). This allowed inferences to be made about the nature of microbial redox reactions, their potential rates, and to verify predictions based on the geochemistry of natural fluids.

In Chapter 3 of this thesis, the identity and activity of microbial communities from these same incubations is determined and discussed. Autotrophic processes were quantified by measuring ${ }^{13} \mathrm{CO}_{2}$ tracer incorporation and active microbes were identified using CARDFISH and 454 sequencing of rRNA amplicons. Epsilonproteobacterial cells completely dominated CARD-FISH counts and were shown by single-cell tracer incorporation (HISH-SIMS; Musat et al., 2008) to be consistently active across all incubation conditions. Furthermore, community composition was correlated with physicochemical conditions, allowing inferences to be made about the factors affecting niche differentiation in natural communities. Finally, the combination of productivity measurements and chemical consumption data allowed the calculation of the efficiency of carbon fixation. In turn, these efficiency data formed the basis of a model that predicted primary productivity in the natural environment.

\subsection{Chapter 4 - Theoretical Investigations into Chemoau- totrophy in Sulfurimonas denitrificans:}

As discussed above, a rough understanding of the metabolism in autotrophic Epsilonproteobacteria is beginning to emerge. At this point, redox reactions used to generate energy are relatively well constrained from pure-culture investigations (Nakagawa and Takai, 2008; Sievert and Vetriani, 2012). However, the precise biochemical mechanisms whereby redox reactions are coupled to energy-conservation and carbon fixation are either very poorly described or not known at all. For example, it is unknown how reversed electron transport produces reductant needed for the rTCA cycle in the autotrophic Campylobacterales (Marshall et al., 2012). Without such a mechanism, these organisms' most fundamental physiological attribute - the fixation of $\mathrm{CO}_{2}$ via the rTCA cycle coupled to sulfur oxidation - cannot be accounted for.

Therefore, the goal of the research presented in Chapter 4 was to provide hypotheses for such knowledge gaps by reviewing available literature and proposing biochemically- 
plausible mechanisms. The organism Sulfurimonas denitrificans was used as a test case, since a well-annotated genome (Sievert et al., 2008) and physiological data were both available (Timmer-Ten Hoor, 1975, 1981; Han and Perner, 2014). Using these data, as well as biochemical knowledge from related organisms, a quantitative in silico model of core metabolism in Sulfurimonas denitrificans was developed. This model is consistent with physiological growth yields, represents the first quantitative metabolic model of an autotrophic epsilonproteobacterium, and has identified plausible novel biochemical mechanisms underlying $S$. denitrificans' growth. Since the model proposes explicit molecular mechanisms, biochemical assays to test model predictions have been proposed. These tests will ultimately reveal the specific mechanisms autotrophic Epsilonproteobacteria use to convert chemical energy into biomass. In turn, understanding these molecular mechanisms will aid in modeling the biogeochemical impact of microbial communities dominated by Epsilonproteobacteria. Finally, investigating the function of these proteins will also provide insight into factors that allowed the adaptive radiation of Epsilonproteobacteria from hydrothermal vents into oxic niches that became available during the rise of oxygen on the ancient Earth. 


\section{Chapter 2}

\section{Assessing Microbial Processes in}

Deep-Sea Hydrothermal Systems by

\section{Incubation at In Situ Temperature}

\section{and Pressure}

This chapter is a paper currently in press at Deep-Sea Research Pt I with co-authors Sean P. Sylva, François Thomas, Craig D. Taylor, Stefan M. Sievert and Jeffrey S. Seewald. The publisher's version can be found at: http://dx.doi.org/10.1016/j.dsr.2016.06.011. A PDF of the final version of this paper will also be deposited at the WHOI Open Access Server (http://darchive.mblwhoilibrary.org/) at a later time. 


\subsection{Abstract}

At deep-sea hydrothermal vents, a large source of potential chemical energy is created when reducing vent fluid and oxidizing seawater mix. In this environment, chemolithoautotrophic microbes catalyze exergonic redox reactions which in turn provide the energy needed to fuel their growth and the fixation of $\mathrm{CO}_{2}$ into biomass. In addition to producing new organic matter, this process also consumes compounds contained both in vent fluid and entrained seawater (e.g. $\mathrm{H}_{2}, \mathrm{NO}_{3}{ }^{-}$). Despite their biogeochemical importance, such reactions have remained difficult to quantify due to methodological limitations. To address this knowledge gap, this study reports a novel application of isobaric gas-tight fluid samplers for conducting incubations of hydrothermal vent fluids at in situ temperature and pressure. Eighteen $\approx 24 \mathrm{~h}$ incubations were carried out, representing seven distinct conditions that examine amendments consisting of different electron donors and acceptors. Microbial activity was observed in all treatments, and time series chemical measurements showed that activity was limited by electron acceptor supply, confirming predictions based on geochemical data. Also consistent with these predictions, the presence of nitrate increased rates of hydrogen consumption and yielded ammonium as a product of nitrate respiration. The stoichiometry of predicted redox reactions was also determined, revealing that the sulfur and nitrogen cycles are incompletely understood at deep-sea vents, and likely involve unknown intermediate redox species. Finally, the measured rates of redox processes were either equal to or far greater than what has been reported in previous studies where in situ conditions were not maintained. In addition to providing insights into deep-sea hydrothermal vent biogeochemistry, the methods described herein also offer a practical approach for the incubation of any deep-sea pelagic sample under in situ conditions. 


\subsection{Introduction}

At deep-sea hydrothermal vents, entire ecosystems are supported by primary production in the absence of sunlight. This process, known as chemosynthesis or chemolithoautotrophy, can occur due to chemical disequilibria between reducing hydrothermal vent fluids and oxidizing seawater. Chemosynthetic microbes catalyze thermodynamically-favorable redox reactions and couple this chemical energy to $\mathrm{CO}_{2}$ fixation, thereby transforming an inorganic energy source into biomass (Jannasch and Mottl, 1985). In addition to supporting productive ecosystems, this process has significant biogeochemical implications. For example, chemosynthetic microbes not only consume reduced inorganic compounds, but they also remove nitrate and oxygen from seawater that mixes with vent fluid.

In the past two decades, the analysis of nucleic acids obtained directly from natural microbial communities as well as the characterization of newly isolated strains of chemolithoautotrophic microbes has revealed insights into the taxonomy, abundance and metabolic potentials of deep-sea vent chemolithoautotrophs (e.g. Huber et al., 2007; Nakagawa and Takai, 2008; Sievert and Vetriani, 2012, and references therein). Studies conducted thus far have shown that fluid composition can exert important controls on microbial community structure and function (Amend et al., 2011; Flores et al., 2011, 2012; Dahle et al., 2015; Hentscher and Bach, 2012), but the reverse question, i.e. how microbes themselves affect fluid composition has received less attention. Although microbial metabolism can be inferred indirectly in low temperature hydrothermal fluids by measuring deviations from the conservative mixing line between high temperature endmember fluids and seawater (Butterfield et al., 2004; Von Damm and Lilley, 2004; Proskurowski et al., 2008; Wankel et al., 2011), this approach cannot provide unequivocal proof that these signatures are microbial nor determine the rates of processes. Therefore, the lack of direct measurements of microbial metabolism hinders our understanding of the biogeochemical role of chemosynthetic processes within vent ecosystems, including their primary productivity (Sievert and Vetriani, 2012).

In order to identify active metabolic pathways and their rates at deep-sea vents, experiments that simulate the natural environment as closely as possible are needed (Sievert and Vetriani, 2012). A conceptually straightforward approach to accomplish this goal is to directly incubate fluids collected from the environment with their resident microbes. This approach is complicated, however, by both inherent challenges of working with deep-sea 
vent fluids as well as difficulties designing experiments to realistically infer in situ processes. Indeed, previous studies have shown that deep-sea microorganisms are sensitive to a decrease in pressure (Bartlett, 2002), suggesting that decompression can affect biological rate measurements. For example, Bianchi et al. (1999) showed that bacterial production rates are underestimated by approximately one half in decompressed pelagic water samples from depths greater than $850 \mathrm{~m}$.

In addition to pressure, the chemical environment is also fundamentally important since the concentration of chemical species directly influences which microbial assimilatory/dissimilatory redox reactions can occur and the rates at which these reactions proceed. The chemical environment in fluid samples collected at deep sea vents may change dramatically if pressure is not maintained during transport to the sea surface due to the loss of volatile species (i.e. $\mathrm{H}_{2}, \mathrm{H}_{2} \mathrm{~S}, \mathrm{CH}_{4}, \mathrm{CO}_{2}$ ). This results in the removal of potential energy sources and alteration of key parameters such as $\mathrm{pH}$, which in turn may affect the composition and activity of the indigenous microbial communities. Artifacts may also be introduced during fluid collection from the natural environment if ambient seawater is entrained. The presence of exogenous seawater not only alters the chemical environment, but also introduces a compositionally distinct microbial community not representative of the vent system.

In addition to technical challenges associated with obtaining representative samples of vent fluid, deriving accurate information on in situ processes from incubations of this fluid is complicated by the fact that chemolithoautotrophic microorganisms will have already affected the geochemistry of fluids prior to sampling, depleting some chemicals while producing others (Butterfield et al., 2004; Von Damm and Lilley, 2004; Proskurowski et al., 2008; Wankel et al., 2011). The extent of this effect is poorly known, but must be a function of fluid flow rate, absolute concentrations of chemical species, and the abundance of microorganisms and their metabolic rates. Because diffuse-flow hydrothermal environments are characterized by a continuous replenishment of substrate-bearing fluids, microbial communities can be sustained in situ even if relevant metabolic redox couples are present at very low concentrations in sampled fluids. Therefore, in closed-system (batch) growth experiments, low concentrations of reactants in energy-yielding redox reactions is problematic since it will be difficult (if not impossible) to measure some processes, despite their likely occurrence in the natural environment.

To circumvent the aforementioned issues, we used an existing hydrothermal fluid sam- 
pler to conduct microbial incubations under simulated sea-floor conditions. Isobaric gas-tight (IGT) fluid samplers (Seewald et al., 2002) were designed to maintain fluids at seafloor pressure following collection. They are ideal for sampling low-flow diffuse vents because their slow fill rate $\left(\approx 75 \mathrm{~mL} \mathrm{~min}^{-1}\right)$ minimizes the entrainment of ambient seawater and a thermocouple co-located with the sampler inlet snorkel provides real-time temperature information to precisely position the fluid intake in the vent environment. As part of this new application, we developed methods for fluid withdrawals from and additions to the IGT sampler, which allowed both initial chemical amendments and monitoring of substrate concentrations and cell numbers over time, all while maintaining seafloor pressure. Below, we report the results of shipboard experiments designed to improve the understanding of microbial metabolism in deep-sea hydrothermal vent fluids by identifying active chemosynthetic redox reactions, inferring the stoichiometry of these reactions, and quantifying their rates.

\subsection{Materials and Methods}

\subsubsection{Field Site}

Vent fluids used for incubation experiments were collected from Crab Spa $\left(9^{\circ} 50.3981 \mathrm{~N}\right.$, $104^{\circ} 17.4942 \mathrm{~W}$ ), a well-studied diffuse-flow hydrothermal vent located on the East Pacific Rise at a depth of 2,506 m, using the ROV Jason II deployed from the R/V Atlantis during research cruise AT26-10 in January 2014. At this site, warm fluids $\left(\approx 24^{\circ} \mathrm{C}\right)$ containing microbes emanate from a well-defined orifice, and have maintained a temporally stable chemical and temperature composition since 2007 (Reeves et al., 2014, Sievert, Seewald Le Bris and Luther, unpublished data). Prior to the first sampling, the site was allowed to stabilize after megafauna (e.g., Riftia pachyptila, Bathymodiolus sp.) were cleared to directly access the fluids emanating from the subseafloor.

\subsubsection{Experimental Design and Methods for Incubations}

Similar to other low temperature vent fluids, many chemical species at Crab Spa are depleted relative to concentrations expected for mixing of the high temperature endmember source fluid with seawater. Therefore, we chose to conduct replicated amendments of these natural vent fluids with electron acceptors and donors (Table 2.1). In particular, dissolved nitrate and oxygen were added to test if their presence would stimulate sulfide oxidation, and 
dissolved hydrogen was added to test whether microbes were capable of hydrogen oxidation, with or without added nitrate. These conditions were compared to controls (no amendments) to confirm that electron donor oxidation was limited by availability of electron acceptors. Since our goal was to mimic the natural environment, most incubations were carried out at $24^{\circ} \mathrm{C}$ in the shipboard laboratory, nearly identical to the temperature at which Crab Spa fluids exit the seafloor. Two additional incubations were carried out at $50{ }^{\circ} \mathrm{C}$ to examine biogeochemical processes at higher temperatures that likely characterize deeper subseafloor environments.

Table 2.1: Summary of conditions during high pressure incubations using IGT fluid samplers

\begin{tabular}{cccccc}
\hline Amendment* & $\begin{array}{c}\text { Concentration } \\
(\mu \mathrm{MM})\end{array}$ & $\begin{array}{c}\text { Temperature } \\
\left({ }^{\circ} \mathrm{C}\right)\end{array}$ & R Replicates & Time from seafloor & Figure \\
Control & $\mathrm{NA}$ & 24 & 3 & $4,7,7$ & $2-2 \mathrm{a}$ \\
$\mathrm{H}_{2}$ & 150 & 24 & 3 & $7,16,16$ & $2-2 \mathrm{~b}$ \\
$\mathrm{NO}_{3}^{-}$ & 100 & 24 & 3 & $5,7,4$ & $2-2 \mathrm{c}$ \\
$\mathrm{O}_{2}$ low & 80 & 24 & 2 & 3,3 & $2-2 \mathrm{~d}$ \\
$\mathrm{O}_{2}$ high & 110 & 24 & 2 & 5,5 & $2-2 \mathrm{~d}$ \\
$\mathrm{NO}_{3}{ }^{-} / \mathrm{H}_{2}$ & $100 / 150$ & 24 & 3 & $7,3,4$ & $2-2 \mathrm{e}$ \\
$\mathrm{NO}_{3}{ }^{-} / \mathrm{H}_{2}$ & $100 / 150$ & 50 & 2 & 4,3 & $2-2 \mathrm{f}$ \\
\hline * in addition to & $13 \mathrm{HCO}_{3}^{-}$ & & & &
\end{tabular}

Prior to deployment at the seafloor, the IGT samplers were washed with dilute $\mathrm{HCl}(\mathrm{pH}$ 3) to remove any residual ${ }^{13} \mathrm{C}$-labeled dissolved inorganic carbonate (DIC) from previous incubations, followed by a $70 \%$ ethanol rinse to sterilize the interior, and acetone to dry the ethanol. A Teflon O-ring was added to the IGT sample chamber prior to deployment to allow fluid stirring following chemical additions and prior to time series sampling during the incubations (Fig 2-1). The snorkel and sample valve dead volume $(\approx 4 \mathrm{ml})$ and sample chamber on the back side of the sample piston were filled with filtered bottom seawater while the accumulator chamber on the backside of the accumulator piston was filled with compressed nitrogen to $\approx 10 \%$ of seafloor pressure at the sampling site (Fig 2-1). During sampling, the sampler inlet valve was opened and fluid entering the sampler forced the sample 
and accumulator pistons to the opposite ends of their respective chambers. Retraction of the accumulator piston compresses nitrogen in the accumulator chamber allowing it to act as a spring that buffers internal pressure fluctuations in the sample chamber caused by external temperature changes during transport from the seafloor to the seasurface. Based on $2^{\circ} \mathrm{C}$ bottom temperature and $25^{\circ} \mathrm{C}$ laboratory temperature, internal pressure increases due to sampler warming are $<15 \%$.

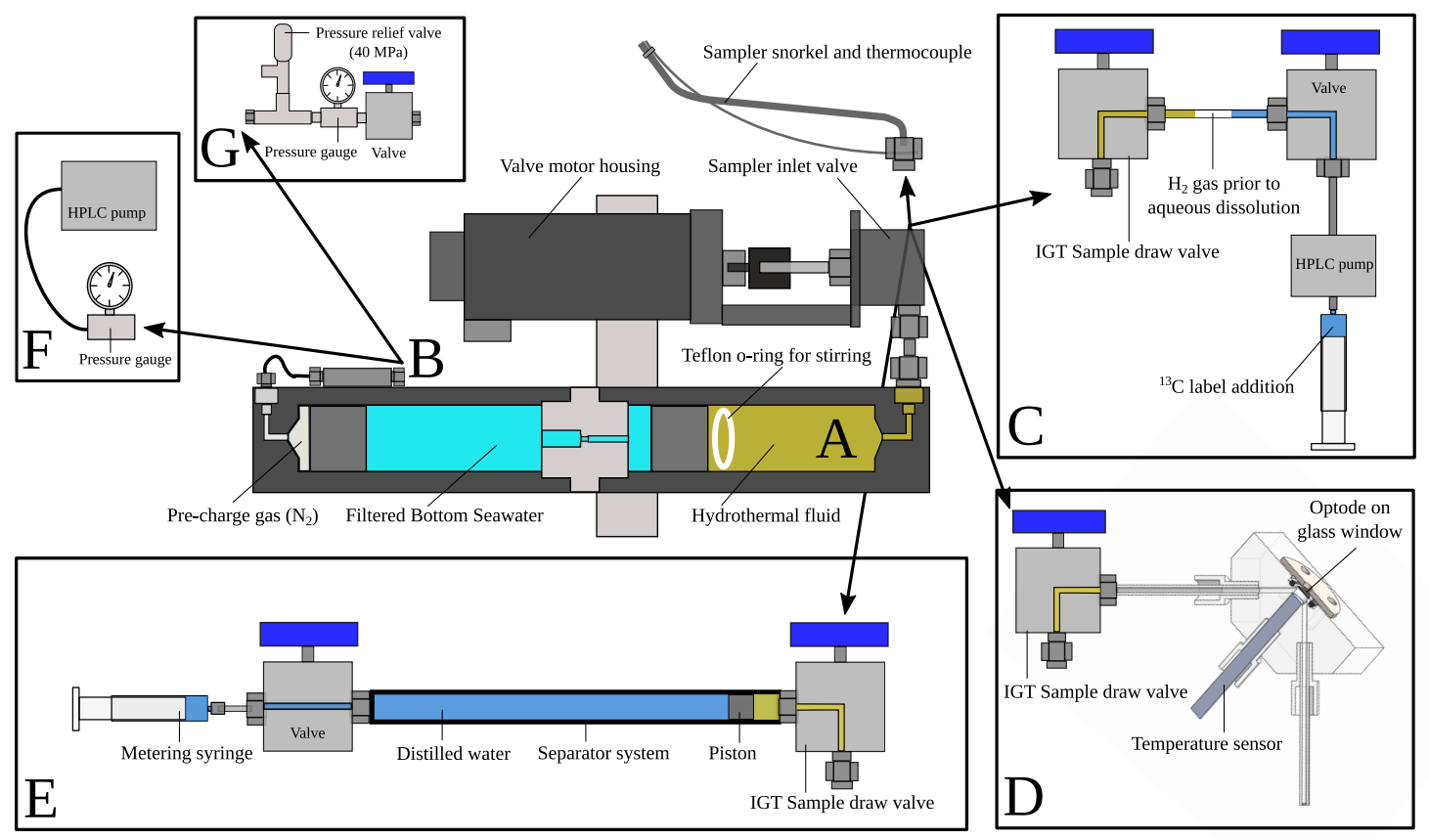

Figure 2-1: Isobaric gas-tight (IGT) sampler configurations used to amend Crab Spa hydrothermal fluids with inorganic substrates, monitor chemical consumption, and track microbial growth. For each incubation, the sample draw valve (shown in panel C, D and E) is attached to the sampler inlet valve and is used to regulate fluid flow during both additions of dissolved chemical species and withdrawals for chemical consumption/cell count measurements. Pressure in the IGT is maintained at seafloor values by means of the pre-charge valve (B) which can be opened to bleed off pressure during amendment addition or maintain pressure by adding fluid using an HPLC pump during sample removal (F). In the case of the addition of dissolved hydrogen gas, the configuration in (C) shows how this gaseous substrate was supplied to the sampler. To make instantaneous measurements of dissolved oxygen from IGT fluids, a fiber-optic sensor was held against the optode window in the flowthrough cell shown in (D). The separator system (E) used for cell counts allows withdrawals of microbial cells from the sampler without exposure to shear stress. (G) shows the safety setup used for high temperature incubations. The operation of all of these components are explained in greater detail in the text.

Vent fluid sampling was performed by positioning the IGT snorkel/thermocouple into the orifice of Crab Spa until temperature readings reached a stable maximum of at least 
$23-24^{\circ} \mathrm{C}$, at which point the sampler inlet valve was opened. The sampler was allowed to fill for $2 \mathrm{~min}$, and then the sampler inlet valve was closed to retain pressure during transit back to the surface. After retrieval of the IGT samplers on board the ship, a limited number of fluid aliquots ( $\approx 10 \mathrm{~mL}$ total volume) were removed for analysis of selected chemical species. Dissolved methane was used as a conservative tracer for the amount of endmember hydrothermal fluid in a given sample. Fluids were not used for incubations if the methane concentration was $<4 \mu \mathrm{M}$, an indication that substantial inadvertent seawater entrainment had occurred during fluid collection. Most shipboard incubations were started within seven hours from sampling at the seafloor, although two IGT samplers used for hydrogen-amended incubations were stored at room temperature for approximately sixteen hours prior to the beginning of the experiment (Table 2.1).

To compensate for pressure loss during fluid withdrawals from the sample chamber, a high performance liquid chromatography (HPLC) pump (Fig 2-1F) was connected to the pre-charge valve (an on/off high-pressure needle valve; Fig 2-1B). Distilled water was then pumped into the accumulator chamber until the desired pressure was reached. For fluid additions to the sample chamber, the HPLC pump was connected to the sampler inlet valve to pump in amendments. To relieve excess pressure caused by fluid additions, the pre-charge valve (Fig. 2-1B) was opened briefly as necessary. In both cases, pressure was monitored using a high-pressure gauge connected to the HPLC pump.

Incubations were initiated by sequentially injecting solutions of known composition. For all additions, the total volume of fluid injected was $\approx 10 \mathrm{~mL}$, which represents $6-7 \%$ of the total volume of the IGT sampler $(150 \mathrm{~mL})$. The first fluid to be injected was $2 \mathrm{~mL}$ of a $\mathrm{NaH}^{13} \mathrm{CO}_{3}\left(99 \%{ }^{13} \mathrm{C}\right.$, Cambridge Isotope laboratories) solution of known concentration made up in $\mathrm{N}_{2}$-purged filtered bottom seawater. This was immediately followed by $8 \mathrm{~mL}$ of filtered bottom seawater containing chemical species for each of the incubations indicated in Table 2.1, at a concentration that would yield the desired abundances after mixing with the vent fluid already in the IGT sample chamber. For all treatments, the final concentration of ${ }^{13} \mathrm{C}$ after injection was $\approx 10 \%$ of the total DIC (supplemental spreadsheet). Immediately after injection, the IGT samplers were rotated $180^{\circ}$ several times to allow the Teflon O-ring to fall through the sample chamber, thereby mixing the fluid contents. Following addition of substrates, fluid chemistry and cell abundance were monitored over an $18-24 \mathrm{~h}$ period by extracting fluid aliquots from the IGT sample chamber after selected time intervals. 
Stock solutions for injection of nitrate, oxygen, and hydrogen were prepared just prior to addition to the IGT sampler. The ${ }^{13} \mathrm{C}$-bicarbonate solution was used within three days of preparation and refrigerated when not in use. Solutions were prepared anoxically in Hungate tubes or Pyrex bottles, and all glassware was muffled at $480^{\circ} \mathrm{C}$ for $4 \mathrm{~h}$ prior to use to remove organic contaminants.

Dissolved oxygen was added as a solution of filtered bottom seawater saturated with ultra-high purity $\mathrm{O}_{2}$ at atmospheric partial pressure $(0.103 \mathrm{MPa})$ for the lower oxygen concentration incubations and in a solution saturated at $0.206 \mathrm{MPa}_{2}$ partial pressure for the higher concentration incubations. Nitrate solutions were prepared from reagent grade $\mathrm{NaNO}_{3}$ dissolved in $\mathrm{N}_{2}$-purged filtered bottom seawater.

Due to the low solubility of hydrogen gas in aqueous solution, a modified approach involving the HPLC pump was used to amend the incubations with dissolved hydrogen (Fig $2-1 \mathrm{C})$. A length of $1 / 8$ stainless steel tubing with an internal volume of $2 \mathrm{~mL}$ was prefilled with hydrogen gas at atmospheric pressure and connected to the IGT sample inlet valve at one end and the HPLC pump outlet at the other. One $\mathrm{mL}$ of the filtered bottom seawater $/{ }^{13} \mathrm{C}$ label solution was then pumped into the length of hydrogen-filled stainless steel tubing before opening the IGT sample valve, which allowed vent fluid into the sample loop, raising the pressure to $25 \mathrm{MPa}$. This solution was allowed to equilibrate for $30 \mathrm{~min}$ so that the compressed hydrogen gas could dissolve into the filtered bottom seawater/vent fluid mixture. Once dissolved, the contents of the $1 / 8$ stainless tube were injected into the sample chamber using the HPLC pump.

Incubations were conducted in the IGT samplers either at in situ vent temperature $\left(\approx 24^{\circ} \mathrm{C}\right.$ in the ship's laboratory) or at $50^{\circ} \mathrm{C}$ by placing them in an oven. Safety considerations necessitated the addition of a $40 \mathrm{MPa}$ pressure-relief connected to the precharge valve to relieve pressure in the event of runaway temperature within the oven (Fig $2-1 \mathrm{G})$. Temperature inside the oven was continuously logged at 5 min intervals for all treatments with iButton temperature recorders (Maxim Integrated, San Jose, CA).

In addition to the unamended controls described above, killed controls were conducted to examine abiotic processes. Abiotic sulfide oxidation with oxygen was tested at $24^{\circ} \mathrm{C}$ and $50^{\circ} \mathrm{C}$ in duplicate experiments by injecting formaldehyde into the IGT sampler to attain a final concentration of $2 \%$, followed by the addition of dissolved oxygen $(>80 \mu \mathrm{M})$ and monitoring the sulfide concentration over time. To test if $\mathrm{H}_{2}$ diffusion or leakage from the 
IGT sampler was occurring, $\mathrm{H}_{2}$ was added to a sampler filled with oxic deionized water and the concentration monitored over time at $24^{\circ} \mathrm{C}$. Both controls were conducted on the same timescale as the actual experiments $(\approx 24 \mathrm{~h})$.

\subsubsection{Analytical Methods:}

Fluid Chemistry: Total dissolved sulfide $\left(\Sigma \mathrm{H}_{2} \mathrm{~S}=\mathrm{H}_{2} \mathrm{~S}+\mathrm{HS}^{-}+\mathrm{S}^{2-}\right)$ was determined potentiometrically using a sulfide-selective electrode. The electrode was calibrated daily with a serial dilution of a standard sodium sulfide solution. $\mathrm{pH}\left(25^{\circ} \mathrm{C}\right)$ was measured in fluids withdrawn from the IGT sampler with a $\mathrm{Ag} / \mathrm{AgCl}$ combination reference electrode calibrated daily. Dissolved methane and hydrogen concentrations were determined shipboard using a gas chromatograph equipped with a $5 \AA$ molecular sieve packed column and serially connected thermal conductivity and flame ionization detectors following quantitative headspace extraction. DIC concentrations (DIC $=\mathrm{CO}_{3}{ }^{2-}+\mathrm{HCO}_{3}{ }^{-}+\mathrm{H}_{2} \mathrm{CO}_{3}{ }^{*}$ ) were determined after fluid acidification with 25 wt. \% phosphoric acid by injecting aliquots of headspace gas directly into a gas chromatograph equipped with a Porapak-Q packed column and a thermal conductivity detector. Dissolved $\mathrm{Mg}, \mathrm{Cl}$ and $\mathrm{SO}_{4}{ }^{2-}$ concentrations were analysed by ion chromatography with suppressed conductivity detection. Dissolved oxygen concentrations in IGT samplers were determined by passing hydrothermal fluid through a custom designed flow-through cell fitted with a commercially available oxygen optode and associated temperature probe (Pts3, Presens, Germany; see Fig 2-1D). The oxygen optode spot was calibrated with oxygen-free water (treated with sodium dithionite) and air-saturated water. Prior to measurements, the optode flow-through cell was flushed with $\mathrm{N}_{2}$-purged filtered bottom seawater or deionized water to remove air bubbles. Oxygen levels were determined in situ by placing an oxygen optode system directly into hydrothermal fluid flow (Model 4330F; Aanderaa Data Instruments, Fall River, MA), which was calibrated by the manufacturer prior to deployment.

For determination of dissolved nitrate and ammonium concentrations, fluids were filtered shipboard through a $0.2 \mu \mathrm{m}$ GTTP membrane (Millipore) and stored frozen at $-20{ }^{\circ} \mathrm{C}$. The concentration of total nitrate + nitrite was determined on shore by conversion to NO and chemiluminescent detection using a NoxBox instrument (Teledyne, San Diego CA, USA) following the original protocol (Garside, 1982). The concentration of total dissolved ammonia $\left(\Sigma \mathrm{NH}_{3}=\mathrm{NH}_{4}{ }^{+}+\mathrm{NH}_{3}\right)$ was determined on filtered samples on board the ship by flow 
injection analysis as previously described (Hall and Aller, 1992).

Fluid aliquots for total cells counts were removed from the IGT samplers using a separator piston system (Fig 2-1E), which permitted withdrawal of fluid from the sample chamber with the sample inlet valve completely open. This strategy prevented the shearing and rupture of cells, which would otherwise have occurred by throttling the sample through the small opening in the sample valve stem that would have been required to maintain a high pressure drop across the valve. Fluids are removed by throttling deionized water on the backside of the separator piston into a metering syringe through an additional high pressure valve attached to the separator tube. Pressure inside the IGT sampler moves the separator piston allowing it to fill with fluid from an incubation, which is then recovered by closing the IGT sample inlet valve, disconnecting the separator and forcing the hydrothermal fluid out with the metering syringe.

Cells were preserved by mixing $1.5 \mathrm{~mL}$ of fluid with $40 \mu \mathrm{L}$ of borate-buffered formalin, stained with $200 \mu \mathrm{L}$ of $0.1 \%$ acridine orange solution (Fisher Scientific), filtered under gentle vacuum onto black $0.2 \mu \mathrm{m}$ polycarbonate filters (GE Healthcare), and enumerated shipboard by fluorescence microscopy (Axioskop 40, Zeiss, Germany). Ten grids were counted per sample and the result extrapolated to the total filtration area to determine the cell concentration. All counts were done in analytical duplicates.

Catalyzed reporter deposition fluorescence in situ hybridization (CARD-FISH): Aliquots of approximately $10 \mathrm{~mL}$ of culture were preserved at $\approx 16$ and $\approx 24 \mathrm{~h}$ after the addition of labels/amendments with paraformaldehyde $(1 \%)$ and kept for $1 \mathrm{~h}$ at room temperature to fix cells. Cells were then filtered under moderate vacuum onto $\mathrm{Au} / \mathrm{Pd}$-sputtered $0.2 \mu \mathrm{m}$ polycarbonate filters (Millipore), washed 2x with $10 \mathrm{~mL} \mathrm{1x}$ PBS, air-dried and stored at $-20^{\circ} \mathrm{C}$ prior to further analysis. Filters were subsequently sectioned, embedded in lowmelting point agarose, and endogenous peroxidases were inactivated by immersion in $3 \%$ $\mathrm{H}_{2} \mathrm{O}_{2}$ for $10 \mathrm{~min}$. Horseradish-peroxidase labeled probes (Biomers) were hybridized at $46^{\circ} \mathrm{C}$ for 3 h. Tyramide amplification with Oregon Green 488-X (Molecular Probes, Inc.) was conducted for $20 \mathrm{~min}$ at $46^{\circ} \mathrm{C}$. Both oligonucleotide probe combinations EPSI549/EPSI914 (Lin et al., 2006; Greuter et al., 2016) and EUBI-III (Daims et al., 1999) were hybridized at $35 \%$ formamide. 


\subsection{Results}

\subsubsection{Crab Spa Microbial Community Composition and Fluid Chemistry:}

All of the Crab Spa fluid samples used for this study had similar chemical concentrations that were consistently different from background seawater (Table 2.2). CARD-FISH counts demonstrated that the microbial communities in the natural fluids were dominated by $E p$ silonproteobacteria ( $\approx 80 \%$ of total cells, Table 2.3 ). Fluids contained elevated levels of methane $(\approx 6 \mu \mathrm{M})$, sulfide $(\approx 190 \mu \mathrm{M})$ and ammonium $(\approx 11.5 \mu \mathrm{M})$, and were slightly acidic $(\mathrm{pH}=5.6)$, as well as being depleted in oxygen and nitrate relative to seawater $(\approx 22$ and $6.8 \mu \mathrm{M}$, respectively). Oxygen concentrations measured in situ were lower than those measured in the IGT samplers $(3.6 \mu \mathrm{M}$ vs $\geq 15 \mu \mathrm{M})$. This excess oxygen can be attributed to air-saturated seawater which was used to fill the sampler dead volume. Small air bubbles entrained in the snorkel tips during vehicle deployment may account for occasional high values observed without oxygen amendments (up to $45 \mu \mathrm{M} \mathrm{O} \mathrm{O}_{2}$ ).

The formation of low temperature vent fluids at oceanic spreading centers occurs as a result of the mixing of high temperature seawater-derived hydrothermal fluids with cold seawater in subseafloor environments (Butterfield et al., 2004; Von Damm and Lilley, 2004). At Crab Spa, a likely candidate for the high temperature endmember is the Tica vent (located $\approx 14 \mathrm{~m} \mathrm{NE}$ of $\mathrm{Crab} S p a$ ) which was venting at $194{ }^{\circ} \mathrm{C}$ during the course of this study. Based on a two-component mixing model for the formation of the Crab Spa fluids involving the endmember Tica vent fluid and seawater, concentrations of dissolved sulfide,

hydrogen, nitrate and oxygen are substantially depleted indicating consumption following mixing in subsurface environments (Table 2.2). 
Table 2.2: Measured and predicted concentrations ${ }^{\mathrm{a}}$ of selected aqueous species at the Crab Spa vent. The endmember concentrations for seawater and the high temperature Tica vent were used to predict the Crab Spa abundances.

\begin{tabular}{|c|c|c|c|c|c|c|c|c|c|c|c|}
\hline & $\begin{array}{c}\mathrm{pH} \\
25^{\circ} \mathrm{C} \\
\end{array}$ & $\begin{array}{l}\mathrm{Mg} \\
\mathrm{mm}\end{array}$ & $\begin{array}{r}\mathrm{H}_{2} \\
\mu \mathrm{M}\end{array}$ & $\begin{array}{l}\mathrm{H}_{2} \mathrm{~S} \\
\mu \mathrm{M}\end{array}$ & $\begin{array}{c}\mathrm{SO}_{4}{ }^{2-} \\
\mathrm{mm}\end{array}$ & $\begin{array}{l}\mathrm{O}_{2} \\
\mu \mathrm{m}\end{array}$ & $\begin{array}{c}\mathrm{NO}_{3}^{-} \\
\mu \mathrm{M}\end{array}$ & $\begin{array}{c}\mathrm{NH}_{4}{ }^{+} \\
\mu \mathrm{m}\end{array}$ & $\begin{array}{c}\mathrm{CH}_{4} \\
\mu \mathrm{M}\end{array}$ & $\begin{array}{l}\text { DIC } \\
\mathrm{mm}\end{array}$ & $\begin{array}{r}\mathrm{Cl} \\
\mathrm{mm}\end{array}$ \\
\hline Tica Endmember & $4.3^{\mathrm{b}}$ & $0^{\mathrm{c}}$ & 410 & 7710 & $<0.5$ & $0^{c}$ & $0^{\mathrm{c}}$ & $2.6^{\mathrm{d}}$ & 113 & 85 & 358 \\
\hline Crab Spa Vent Fluid ${ }^{\mathrm{e}}$ & 5.6 & 49.2 & $<2$ & 184 & 26.5 & $3.6^{\mathrm{f}}$ & 6.3 & 11.9 & 6.3 & 8.2 & 546 \\
\hline Crab Spa Predicted & - & 49.2 & 29 & 552 & 25.8 & 107 & 32 & 0.2 & 8.1 & 8.2 & 526 \\
\hline Bottom Seawater & 7.9 & 53.0 & $<2$ & $<1$ & 27.7 & 115 & 34 & 0 & $<0.002$ & 2.3 & 539 \\
\hline
\end{tabular}

${ }^{\mathrm{a}} \mathrm{mm}=\mathrm{mmol} / \mathrm{kg}$ fluid, $\mu \mathrm{m}=\mu \mathrm{mol} / \mathrm{kg}$ fluid, $\mathrm{mM}-\mathrm{mmol} / \mathrm{L}$ fluid, $\mu \mathrm{M}=\mu \mathrm{mol} / \mathrm{L}$ fluid.

品 bot extrapolated to zero-Mg. Measured in sample containing $12.4 \mathrm{~mm} \mathrm{Mg}$.

${ }^{c}$ Assumed endmember value for high-temperature Tica vent fluids.

${ }^{\mathrm{d}}$ Data from Reeves et al. (2014).

${ }^{\mathrm{e}}$ Corrected for sampler dead volume except for $\mathrm{pH}$

${ }^{\mathrm{f}}$ Measured in situ with oxygen optode system. 
Table 2.3: Percentage of DAPI-stained cells hybridizing to specific CARD-FISH probes in Crab Spa vent fluids and experimental amendments of Crab Spa fluids after 16-24 h of incubation. Values shown are averages from 2-3 biological replicates with the standard deviation $(\mathrm{n}=3)$ or range $(\mathrm{n}=2)$ as errors.

\begin{tabular}{ccc}
\hline Treatment & $\begin{array}{c}\text { EUBI-III-hybridized cells } \\
(\% \mathrm{DAPI})\end{array}$ & $\begin{array}{c}\text { EPSI549/914-hybridized cells } \\
\text { (\% DAPI })\end{array}$ \\
\hline Crab Spa fluid, no incubation $(\mathrm{n}=2)$ & $93.6 \pm 1.0$ & $80.3 \pm 4.2$ \\
Control, $24^{\circ} \mathrm{C}(\mathrm{n}=3)$ & $94.0 \pm 4.2$ & $87.9 \pm 18.4$ \\
$\mathrm{H}_{2}$ amend., $24^{\circ} \mathrm{C}(\mathrm{n}=3)$ & $94.2 \pm 6.4$ & $81.8 \pm 14.8$ \\
$\mathrm{NO}_{3}{ }^{-}$amend., $24^{\circ} \mathrm{C}(\mathrm{n}=3)$ & $100.7 \pm 1.4$ & $97.8 \pm 2.7$ \\
$\mathrm{O}_{2}$ amend. $80 \mu \mathrm{M}, 24^{\circ} \mathrm{C}(\mathrm{n}=2)$ & $96.5 \pm 0.3$ & $100.1 \pm 2.8$ \\
$\mathrm{O}_{2}$ amend. $110 \mu \mathrm{M}, 24^{\circ} \mathrm{C}(\mathrm{n}=2)$ & $96.2 \pm 1.9$ & $99.2 \pm 0.1$ \\
$\mathrm{NO}_{3}{ }^{-} / \mathrm{H}_{2}$ amend., $24^{\circ} \mathrm{C}(\mathrm{n}=3)$ & $99.0 \pm 0.5$ & $100.2 \pm 4.3$ \\
$\mathrm{NO}_{3}{ }^{-} / \mathrm{H}_{2}$ amend., $50^{\circ} \mathrm{C}(\mathrm{n}=2)$ & $95.8 \pm 1.4$ & $97.2 \pm 3.6$ \\
\hline
\end{tabular}

\subsubsection{Incubation results:}

In all cases, the natural microbial community remained dominated by Epsilonproteobacteria, and in some cases, showed an enrichment compared to background samples (Table 2.3). Below, we detail specific observations for each condition.

Control treatment (Fig 2-2a): In this case, the only addition to fluids was a ${ }^{13} \mathrm{C}$ bicarbonate label. Although cell numbers did not change consistently during incubation, a clear and replicable pattern in chemical concentrations was observed. Sulfide dropped by $\approx 100$ $\mu \mathrm{M}$ in all replicates and stabilized at $90-100 \mu \mathrm{M}$ for the remainder of the experiment, while low initial levels of nitrate and oxygen decreased below the detection limits of $\approx 1$ and $\approx 5$ $\mu \mathrm{M}$, respectively. Ammonium concentrations decreased initially by $5-10 \mu \mathrm{M}$ in two of the replicates, which were elevated at the beginning of the incubation for unknown reasons.

Hydrogen additions (Fig 2-2b): The hydrogen-amended incubations were characterized by a pattern of chemical depletion that was similar to the control treatment. In this case, sulfide concentrations decreased by $\approx 100 \mu \mathrm{M}$ initially, and neither hydrogen nor sulfide were exhausted during the incubation period. Hydrogen decreased slowly in all three replicates, with a concomitant increase in sulfide for the last three time points. Cell concentrations 
either did not show a consistent trend or increased gradually. Ammonium increased slightly in two replicates and decreased slightly in one, and nitrate and oxygen were exhausted rapidly as in the control treatment to concentrations below their detection limits. Initial sulfide concentrations were lower for the two IGT sampler incubations that were started after $\mathrm{a} \approx 10$ h delay.

Nitrate additions (Fig 2-2c): Upon the addition of nitrate, sulfide concentrations decreased to below detection after $\approx 12-18 \mathrm{~h}$. Oxygen was fully depleted after the second time point, and nitrate concentrations gradually decreased throughout the incubations. Ammonium increased in two out of three replicates and cell concentrations either increased gradually during incubations or showed no consistent pattern.

Oxygen additions (Fig 2-2d): For the oxygen amended incubations conducted at both 80 and $110 \mu \mathrm{M}$, sulfide and oxygen reached levels below detection after 12-18 h. Cell counts were consistent between replicates with an initial increase at $\approx 80 \mu \mathrm{M}$ after $7 \mathrm{~h}$ and a continuous increase at $\approx 110 \mathrm{\mu M}$ throughout the duration of the incubation. Nitrate was eventually depleted across all treatments, although a transient increase was observed in both $\approx 80 \mu \mathrm{M}$ oxygen replicates. Ammonium decreased in all incubations by $\approx 5 \mu \mathrm{M}$.

Nitrate/hydrogen additions at $24 \not{z} C$ (Fig 2-2e): These incubations were characterized by a complete exhaustion of hydrogen, sulfide, and oxygen and a gradual decrease in nitrate. A consistent and replicable increase in ammonium of around $20 \mu \mathrm{M}$ was observed for all replicates, and cell concentrations increased from the first time point for all incubations, though later decreased in two cases.

Nitrate/hydrogen additions at $50 \check{Z} C$ (Fig 2-2f): In these treatments, a continuous increase in cell numbers was observed, accompanied by complete exhaustion of oxygen, hydrogen, sulfide and a large decrease in nitrate. A large increase in ammonium of $\approx 60 \mu \mathrm{M}$ was observed for both replicates.

Killed controls at 24 and $50^{\circ} \mathrm{C}$ (supplemental spreadsheet): For two killed controls at $24^{\circ} \mathrm{C}$, sulfide decreased between $6-20 \mu \mathrm{M}$ over a $17 \mathrm{~h}$ period, whereas at $50^{\circ} \mathrm{C}$, sulfide either decreased by $\approx 30 \mu \mathrm{M}$ or increased slightly $(18 \mu \mathrm{M})$ over a $12 \mathrm{~h}$ period. Although oxygen concentrations did decrease in some controls, levels never dropped below $94 \mu \mathrm{M}$. 
A - Control, $24^{\circ} \mathrm{C}$
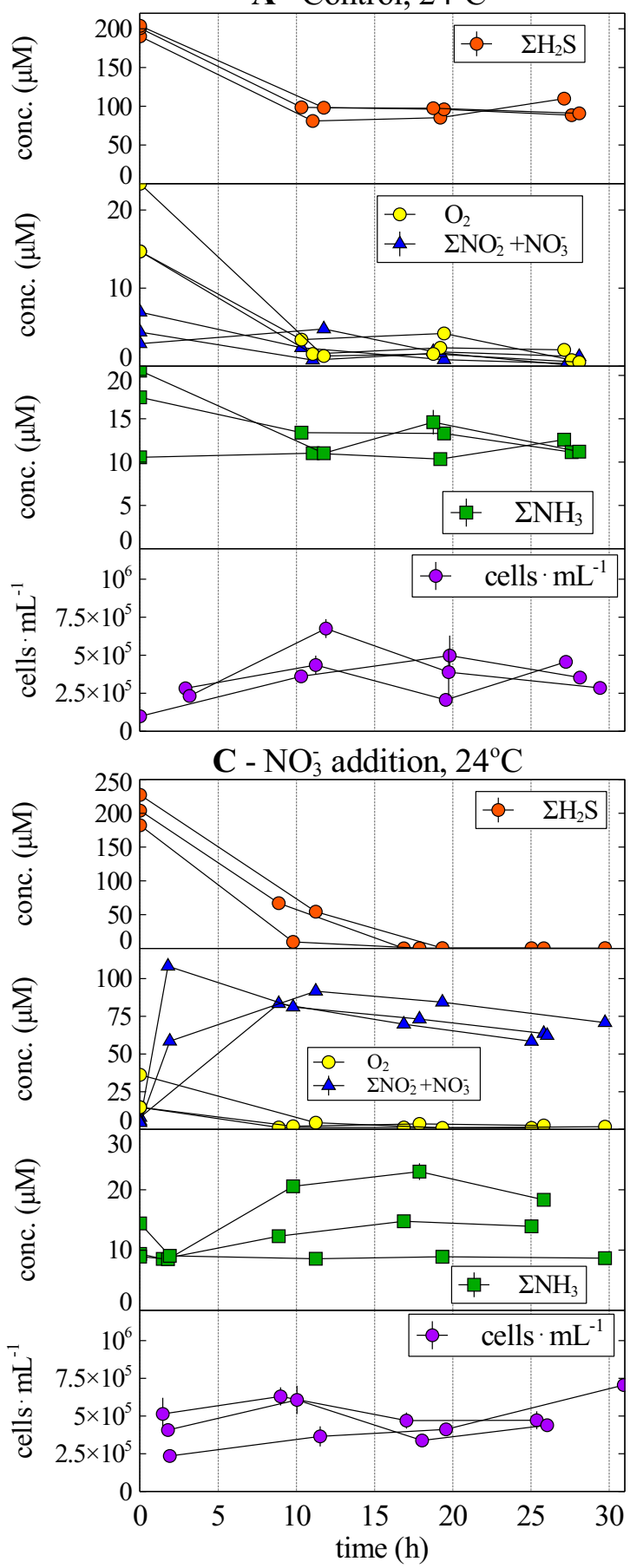

B $-\mathrm{H}_{2}$ addition, $24^{\circ} \mathrm{C}$

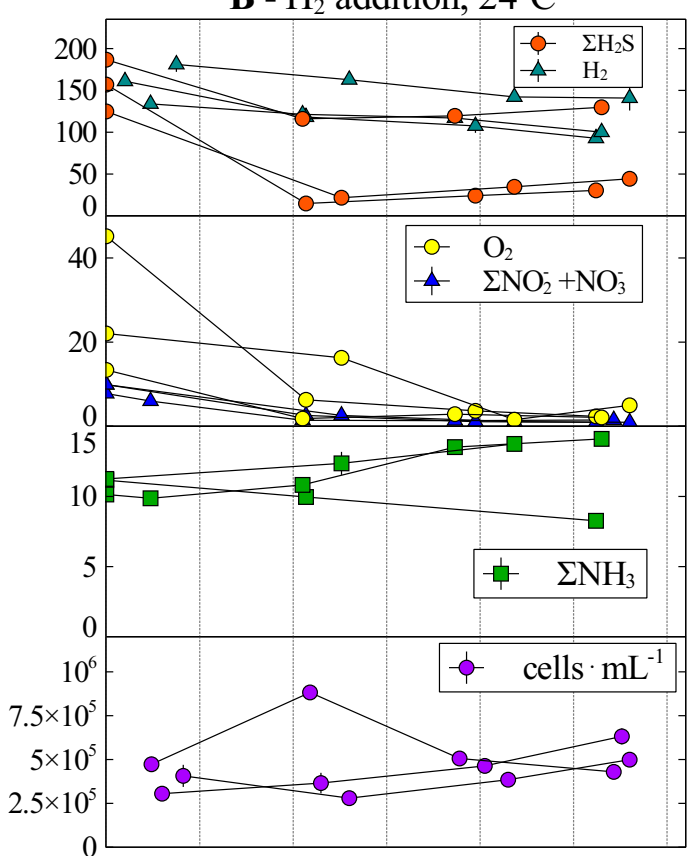

D - $\mathrm{O}_{2}(80 \& 110 \mu \mathrm{M}), 24^{\circ} \mathrm{C}$

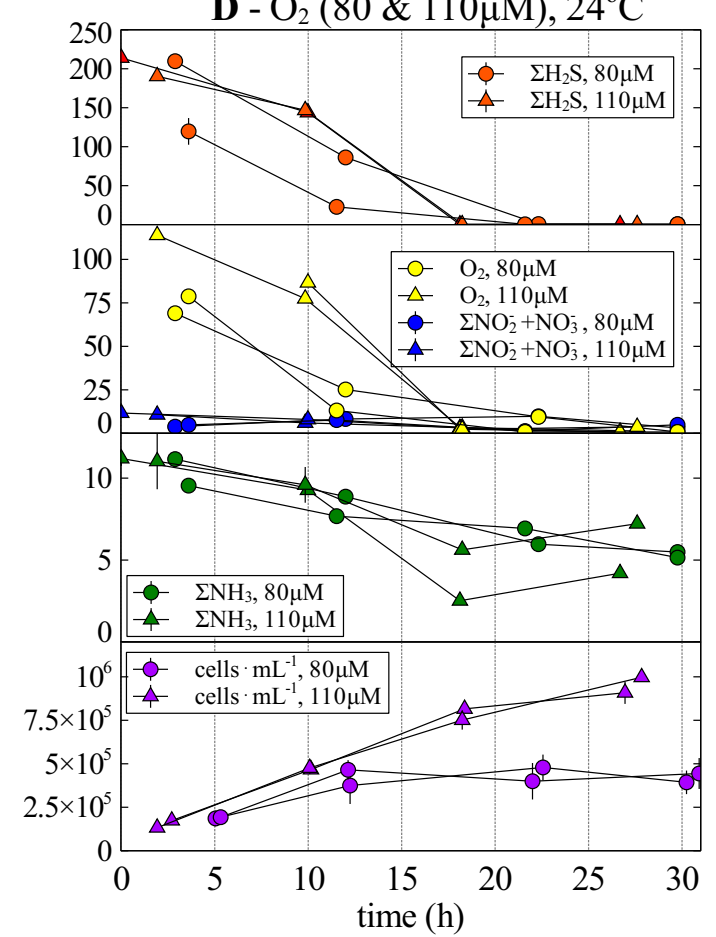



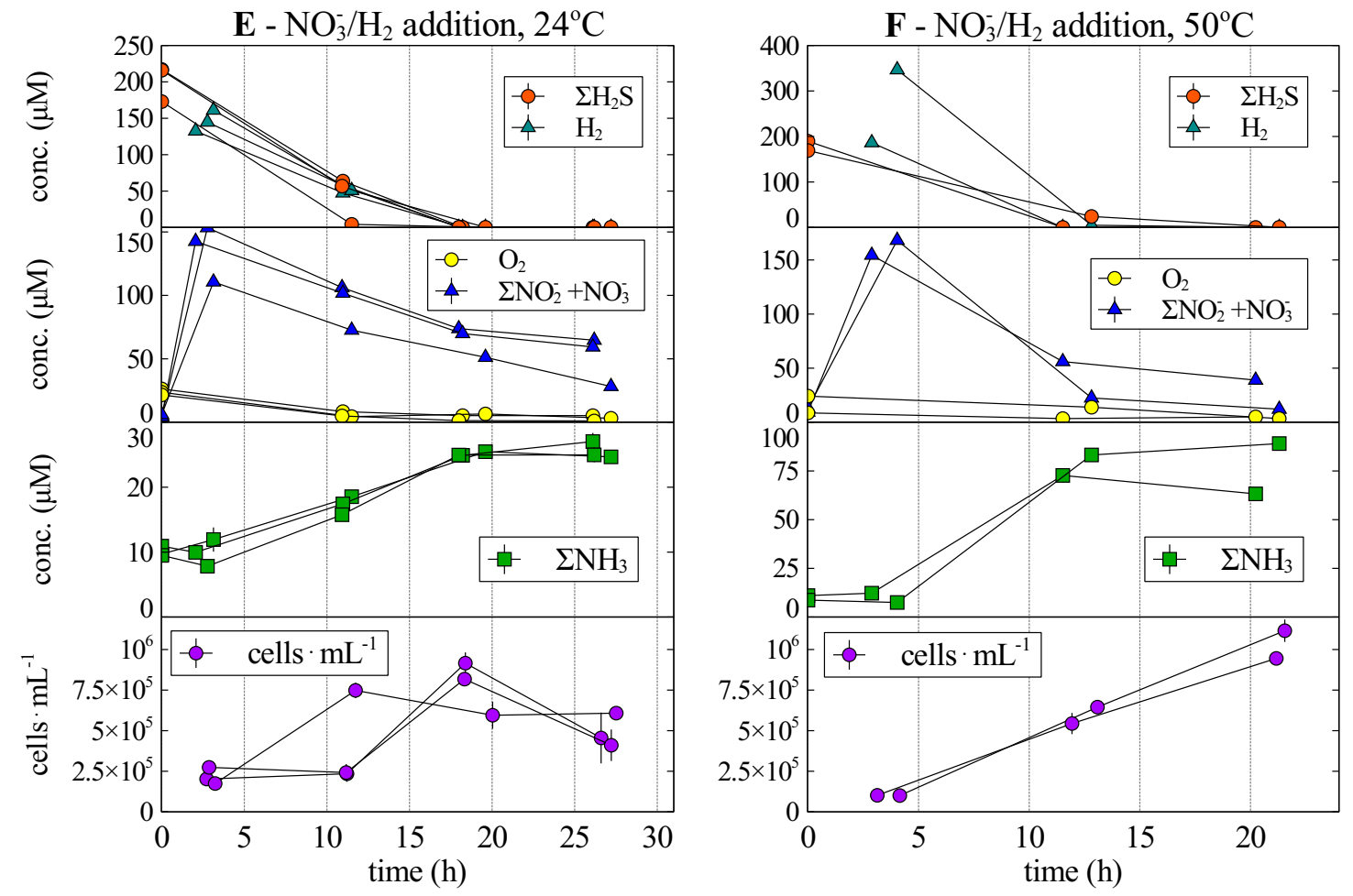

Figure 2-2: Incubation results for 6 different treatment types. With the exception of (f), all incubations were carried out at $24{ }^{\circ} \mathrm{C}$. a) Control, only ${ }^{13} \mathrm{HCO}_{3}{ }^{-}$label in anaerobic filtered bottom seawater, b) Hydrogen additions, c) Nitrate additions, d) Oxygen additions, e) Nitrate/hydrogen additions, f) Nitrate/hydrogen additions $\left(50^{\circ} \mathrm{C}\right)$. For the oxygen additions, triangles indicate the higher oxygen treatment $(110 \mu \mathrm{M})$ and circles denote the lower oxygen treatment $(80 \mu \mathrm{M})$. 


\subsection{Discussion:}

Our study quantified concentrations of redox reactive species and cellular abundance during short-term laboratory incubations of deep-sea hydrothermal vent fluid, allowing biogeochemical processes to be carefully tracked (Fig 2-2). Since these incubations were carried out at deep-sea pressure and temperature with $\mu \mathrm{M}$ concentrations of amendments (Table 2.1), conditions were only slightly altered from the natural environment. Preservation of natural microbial community structure during the incubations (Table 2.3) suggests that our data may accurately reflect processes occurring in the natural environment. Below, we discuss how the incubation results can be used to confirm hypotheses derived from observations of in situ chemistry, infer the stoichiometry of microbially-mediated processes by quantifying redox reactions catalyzed by the community, and determine the rates of microbial processes (Table 2.4).

\subsubsection{Chemosynthesis at Crab Spa}

Models for the formation of low temperature ridge-crest hydrothermal fluids involve twocomponent mixing of a high temperature Mg-free endmember hydrothermal fluid with Mgrich (53 mM Mg) seawater. Within the context of such a model, the $\mathrm{Mg}$ content of low temperature fluids provides a direct constraint on the relative contributions of the hightemperature endmember and seawater. The Mg content of the Crab Spa fluid (49.3 mM) indicates mixing between seawater and hydrothermal end-member fluid at a ratio of approximately 14:1 (Table 2.2). This mixing ratio has remained relatively constant over time at Crab Spa, since it has been observed that fluids have maintained similar temperature, chemistry and microbial composition since 2007 (Reeves et al., 2014, Sievert, Seewald Le Bris and Luther, unpublished data). The fluids also carry a strong imprint of subseafloor microbial activity as evidenced by depletions of redox sensitive chemical species such as oxygen, nitrate, sulfide, and hydrogen relative to values predicted for conservative mixing, and enrichment of other species such as ammonium (Table 2.2). Supporting this presumed microbial activity, both freshly sampled and incubated fluids were dominated by Epsilonproteobacteria (Table 2.3), which are well-known to utilize or produce these compounds during their metabolism (Sievert and Vetriani, 2012; Nakagawa and Takai, 2008). 
Table 2.4: Summary of rates of change in chemical concentrations and cell densities. N.A. $=$ Not applicable, process not observed. N.R. $=$ Data not reported. In these cases data is not presented because the resolution of time series measurements is too low to capture accurate rates. This is due to the low initial concentrations of the analyte in question. Cellspecific rates during incubations were calculated by dividing volumetric rates by the average of initial and maximum cell densities.

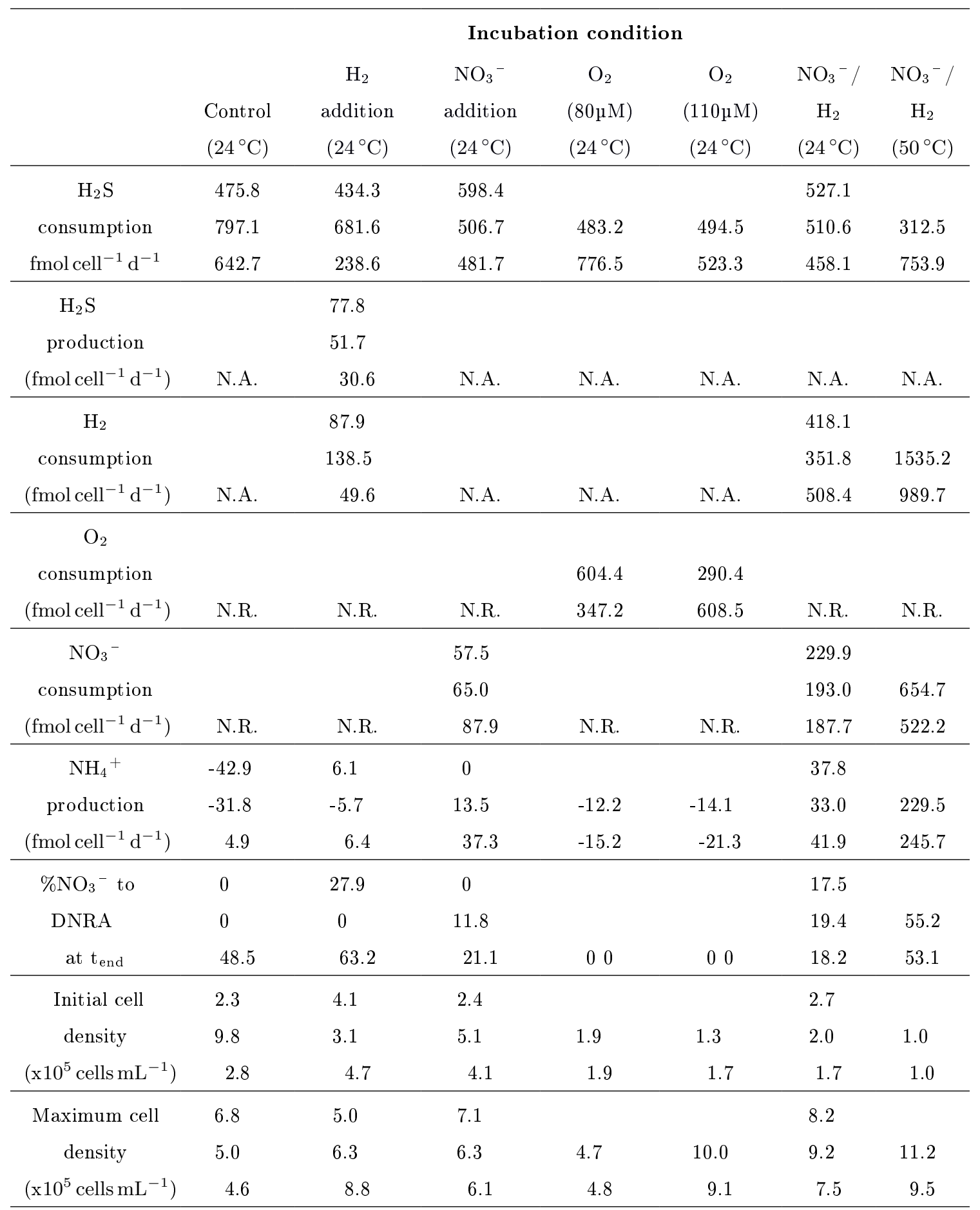


Although sulfate and carbon dioxide are quantitatively the most abundant electron acceptors in these fluids, nitrate, oxygen and intermediate sulfur species (i.e. elemental sulfur, polysulfides) are the only electron acceptors present that are known to be utilized by autotrophic Epsilonproteobacteria to generate energy in combination with sulfide or hydrogen as electron donors (Sievert and Vetriani, 2012)(Fig 2-2). Redox reactions involving these chemical species likely support autotrophic microbes in situ, but evidence is currently lacking for which are preferentially utilized by microbes and which chemicals ultimately limit energy-generating chemosynthetic reactions. Important clues about these questions can be found in the chemistry of Crab Spa fluids, where both oxygen and nitrate are depleted relative to predictions based on conservative mixing, while sulfide remains high $(\approx 190 \mu \mathrm{M})$. This depletion of electron acceptors is most apparent for oxygen, where in situ measurements yielded a value of $3.6 \mu \mathrm{M}, \approx 103 \mu \mathrm{M}$ less than predicted for conservative mixing (Table 2.2).

While oxygen and nitrate concentrations measured in situ are very low, they would still be more than sufficient to support chemosynthesis in the natural environment if they were available to microbes at these levels. However, since oxygen was measured in exiting fluids just outside the vent orifice, turbulent mixing with ambient seawater could have introduced oxygen not present in the subseafloor. In addition, even if $3.6 \mu \mathrm{M}$ is an accurate value for subseafloor fluids, it is likely that there are microenvironments in which these reactants are further depleted, such as in biofilms in the subsurface biosphere. In such an environment, high densities of microbes would likely draw nitrate and oxygen down to much lower levels and ultimately the diffusion of these electron acceptors would limit energy-generating chemosynthetic reactions despite electron donors being present at high concentrations.

Our results support this conjecture by clearly showing that sulfide cannot be completely consumed during experiments (Fig 2-2a, Fig 2-2b) without the addition of either nitrate or oxygen (Fig 2-2c-f). The observed depletions in dissolved sulfide are unlikely to be the result of abiotic oxidation with $\mathrm{O}_{2}$ since killed controls showed no or very low levels of sulfide and oxygen consumption (see supplementary spreadsheet), consistent with other studies assessing the role of biotic and abiotic processes in sulfide oxidation (Luther et al., 2011). In addition, robust growth of Epsilonproteobacteria was associated with sulfide consumption in the presence of oxygen (Fig 2-2d, Table 2.3), strongly suggesting that microbial sulfide oxidation is tied to oxygen in these incubations (see Table 2.5 for likely metabolic 
reactions). In contrast to the oxygen addition experiments, nitrate additions did not result in a consistent pattern of growth, despite rapid and complete consumption of sulfide and enrichment of Epsilonproteobacteria compared to background samples (Table 2.3). This observation, combined with the fact that nitrate was consumed more slowly than oxygen (Table 2.4), suggests that oxygen serves as the preferred electron acceptor in situ, in line with higher growth yields predicted for oxygen relative to nitrate Chen and Strous (2013), a phenomenon that has been observed previously in chemostat cultures of Epsilonproteobacteria Timmer-Ten Hoor (1981).

In addition to sulfide, hydrogen oxidation also has the potential to be an important process supporting chemolithoautotrophic production at vents, since it can support higher growth rates and/or yields than sulfide oxidation in autotrophic Epsilonproteobacteria (Takai et al., 2006; Han and Perner, 2014). Although present in endmember fluids, hydrogen is barely detectable upon sampling at Crab Spa (Table 2.2), suggesting that microbes in the subseafloor are actively consuming it by coupling its oxidation to either oxygen, nitrate or sulfur reduction. Supporting this idea, hydrogen was rapidly and completely consumed in incubations that also received nitrate (Fig 2-2e, f), and consumed more slowly in incubations to which only hydrogen was added (Fig 2-2b). As with sulfide, this oxidation was almost certainly microbially-mediated since abiotic $\mathrm{H}_{2}$ oxidation by oxygen is kinetically inhibited at the incubation temperatures Foustoukos et al. (2011). It is noteworthy that drawdown of hydrogen continued after the depletion of nitrate/oxygen and was accompanied by a steady increase in the concentration of sulfide (Fig 2-2). This increase in sulfide was most likely due to the oxidation of hydrogen coupled to the respiration of oxidized sulfur species. Although dissimilatory sulfate reduction could potentially result in such sulfide production, it is unlikely to have occurred in these incubations since this process has not been reported in Epsilonproteobacteria, which dominated the incubations (Table 2.3). In contrast, there is ample evidence that Epsilonproteobacteria can catalyze the oxidation of hydrogen coupled to the respiration of intermediate sulfur species such as polysulfide, elemental sulfur or thiosulfate (Hedderich et al., 1998; Mino et al., 2014; Sievert and Vetriani, 2012, and references therein). 
Table 2.5: Important predicted redox reactions in incubations of Crab Spa fluids that are most likely microbially catalyzed. These reactions are used in subsequent calculations of redox stoichiometry (see Methods and Discussion).

\begin{tabular}{|c|c|c|c|}
\hline Equation \# & Predicted microbially catalyzed reaction & Description & Evidence for reaction \\
\hline 1 & $2 \mathrm{H}_{2} \mathrm{~S}+\mathrm{O}_{2} \longrightarrow 2 \mathrm{~S}^{0}+2 \mathrm{H}_{2} \mathrm{O}$ & $\begin{array}{l}\text { Sulfide oxidation to sulfur } \\
\text { coupled to oxygen reduction }\end{array}$ & $\mathrm{O}_{2}$ additions \\
\hline 2 & $2.5 \mathrm{H}_{2} \mathrm{~S}+\mathrm{H}^{+}+\mathrm{NO}_{3}^{-} \longrightarrow 2.5 \mathrm{~S}^{0}+3 \mathrm{H}_{2} \mathrm{O}+0.5 \mathrm{~N}_{2}$ & $\begin{array}{l}\text { Sulfide oxidation to sulfur } \\
\text { coupled to denitrification }\end{array}$ & $\mathrm{NO}_{3}{ }^{-}$additions \\
\hline 3 & $4 \mathrm{H}_{2} \mathrm{~S}+4 \mathrm{H}^{+}+\mathrm{NO}_{3}^{-} \longrightarrow 4 \mathrm{~S}^{0}+3 \mathrm{H}_{2} \mathrm{O}+2 \mathrm{H}^{+}+\mathrm{NH}_{4}^{+}$ & $\begin{array}{l}\text { Sulfide oxidation to sulfur } \\
\text { coupled to DNRA* }\end{array}$ & $\mathrm{H}_{2}$ and $\mathrm{NO}_{3}{ }^{-}$additions \\
\hline 4 & $\mathrm{H}_{2}+\mathrm{O}_{2} \longrightarrow 2 \mathrm{H}_{2} \mathrm{O}$ & $\begin{array}{c}\text { Hydrogen oxidation } \\
\text { coupled to oxygen reduction }\end{array}$ & $\mathrm{H}_{2}$ and $\mathrm{H}_{2} / \mathrm{NO}_{3}{ }^{-}$additions \\
\hline 5 & $2.5 \mathrm{H}_{2}+\mathrm{H}^{+}+\mathrm{NO}_{3}{ }^{-} \longrightarrow 3 \mathrm{H}_{2} \mathrm{O}+0.5 \mathrm{~N}_{2}$ & $\begin{array}{c}\text { Hydrogen oxidation } \\
\text { coupled to denitrification }\end{array}$ & $\mathrm{H}_{2}$ and $\mathrm{H}_{2} / \mathrm{NO}_{3}{ }^{-}$additions \\
\hline 6 & $4 \mathrm{H}_{2}+2 \mathrm{H}^{+}+\mathrm{NO}_{3}^{-} \longrightarrow 3 \mathrm{H}_{2} \mathrm{O}+\mathrm{NH}_{4}^{+}$ & $\begin{array}{l}\text { Hydrogen oxidation } \\
\text { coupled to DNRA* }\end{array}$ & $\mathrm{H}_{2}$ and $\mathrm{H}_{2} / \mathrm{NO}_{3}{ }^{-}$additions \\
\hline 7 & $\mathrm{H}_{2}+\mathrm{S}^{0} \longrightarrow \mathrm{H}_{2} \mathrm{~S}$ & $\begin{array}{l}\text { Hydrogen oxidation } \\
\text { coupled to sulfur reduction }\end{array}$ & $\mathrm{H}_{2}$ additions \\
\hline
\end{tabular}

* DNRA $=$ Dissimilatory nitrate reduction to ammonium 
However, it should be noted that sulfur species with intermediate oxidation states are likely quantitatively less important than oxygen or nitrate as electron acceptors in situ, since sulfide production was only observed at hydrogen concentrations 3-4 times the maximum predicted for the natural environment (Table 2.2), and only after oxygen and nitrate were both depleted.

Together, these results provide direct evidence that depletions of redox-active chemicals at Crab Spa are microbially mediated, and that the supply of electron acceptors limits chemosynthesis in situ. Although methane is present at $\approx 6 \mu \mathrm{M}$ in Crab Spa fluids, it was not consumed during incubation experiments even at high oxygen tensions (data not shown). The lack of methane oxidation could be due to the competitive exclusion of methanotrophic communities in situ by fast-respiring Epsilonproteobacteria drawing oxygen and nitrate down to levels below which aerobic or denitrifying methanotrophs could survive. In this case, the sampled fluids in our study would not contain large populations of methanotrophs. This appears to be the case at the Menez Gwen hydrothermal vent field, where metagenomes indicate the absence of known methane-oxidizing genes (Meier et al., 2016), despite the end-member fluids being rich in methane (Charlou et al., 2000).

\subsubsection{Biogeochemistry of Sulfur and Nitrogen:}

While the above discussion paints a general picture of processes underlying chemosynthesis at Crab Spa, incubation results can also be used to determine the stoichiometry of oxidation and reduction reactions catalyzed by microbes, which in turn constrains possible biochemical mechanisms. For example, in all incubations, the amount of nitrate and oxygen consumed was never sufficient to account for the complete oxidation of sulfide to sulfate (supplemental spreadsheet), suggesting that sulfide was oxidized to an intermediate oxidation state sulfur species. Some autotrophic Epsilonproteobacteria can catalyze sulfide oxidation to elemental sulfur $\left(\mathrm{S}^{0}\right)$ (Wirsen et al., 2002; Sievert et al., 2007), and indeed white flocculent material (likely composed of $\mathrm{S}^{0}$ ) continuously emanates from Crab Spa.

In order to test whether this was occurring in our incubations, the overall redox balance between the total amounts of electron donors and acceptors consumed was determined. This was accomplished by using the reactions summarized in Table 2.5 to calculate the ratio of electrons transferred from electron donor to acceptor as indicated by changes in the concentrations of relevant chemical species. A ratio of one indicates a balance between 
the consumption of electron donors and acceptors, and examination of Fig 2-3 reveals that the only treatment where electron donors and acceptors were balanced was for the oxygen additions. In contrast, values greater than one were observed for all of the other conditions, indicating that the amount of electrons predicted to be transferred due to oxidation of electron donors exceeds the amount that can be accounted for by the changes in the concentrations of electron acceptors - even assuming that sulfide was oxidized to the intermediate $\mathrm{S}^{0}$. Although alternative electron acceptors used in microbial respiration could

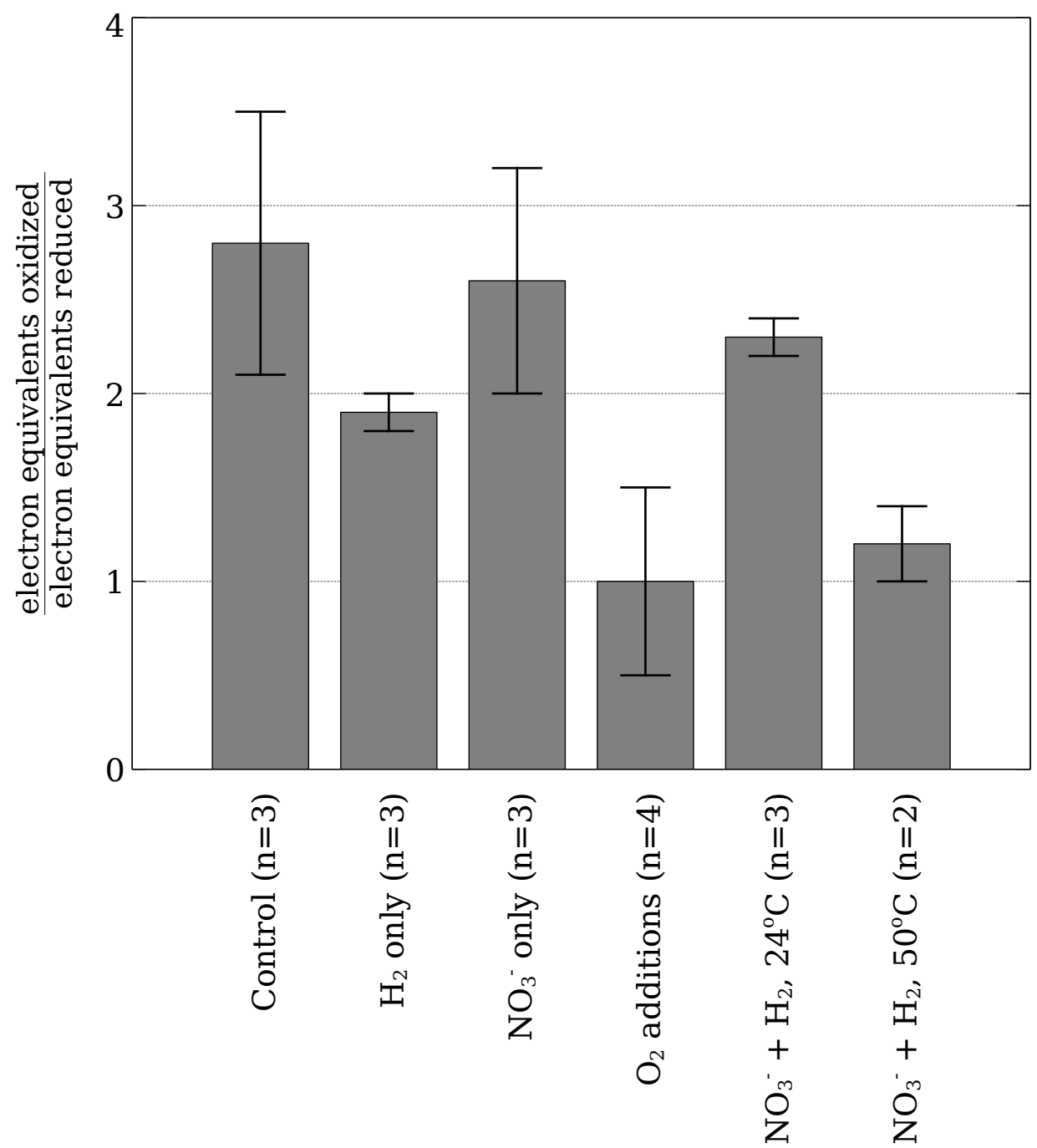

Figure 2-3: Community redox balance for IGT incubations represented as a ratio. A ratio of one indicates balanced use of electron donors and acceptors and a ratio greater than one indicates that more electron donor was consumed than could be accounted for by consumption of electron acceptors (according to the assumptions outlined in the text). Errors are either standard deviations $(n>2)$, or ranges $(n=2)$. 
potentially account for this imbalance, we know of no other candidate electron acceptors at Crab Spa that could support additional sulfide oxidation.

However, an alternative possibility which can consume sulfide without requiring additional microbial respiration is the abiotic production of polysulfide by reaction of sulfide with elemental sulfur. As mentioned above, elemental sulfur is probably present in fluids upon sampling, which would therefore be available to react with sulfide and contribute to the discrepancy observed in Fig 2-3. Because the oxidation state of polysulfide varies as a function of chain length, its presence could explain both the high redox balance ratios and inter-sample variability observed in Fig 2-3. In addition to this completely abiotic mechanism, this process may be partly biologically-mediated. Polysulfide has been shown to be produced during sulfur oxidation by Beggiatoa sp (Berg et al., 2014) and has been implicated in the reaction mechanism of sulfide:quinone oxidoreductase (Griesbeck et al., 2002), which is thought to be used by some Epsilonproteobacteria to oxidize sulfide (Yamamoto and Takai, 2011). In this biologically-mediated process, elemental sulfur produced by microbes during oxidation of sulfide presumably reacts with surrounding sulfide molecules, thereby consuming more sulfide without requiring any extra respiratory electron acceptor to be present.

As discussed above, oxygen is likely the preferred electron acceptor for microbial communities at Crab Spa. As a result, oxygen is highly depleted in situ (Table 2.2), and nitrate may serve as an important alternative electron acceptor. This role for nitrate as a secondary electron acceptor is consistent with its consumption during several incubations to which no oxygen was added (Fig 2-2c, 2e, 2f). Because the method used in this study to quantify nitrate consumption actually measures the sum of nitrate + nitrite, the observed nitrate depletions (Fig 2-2) indicate that nitrate was reduced beyond nitrite to either nitric oxide, nitrous oxide, nitrogen, or ammonium. Since the concentration of ammonium was also determined, our data provide a direct means to assess the relative importance of denitrification versus dissimilatory reduction to ammonium (DNRA), the two major nitrate removal processes expected in this system. Ammonium, which is already present at elevated levels in Crab Spa fluids (11.5 $\mu \mathrm{M}$, Table 2.2), increased in some incubations when either nitrate or hydrogen was added alone and in one control, suggesting slow rates of DNRA in these cases (Table 2.4, Fig 2-2a, b, c). However, the amount of nitrate converted to ammonium was $\leq$ $9.4 \mathrm{\mu M}$ and highly variable, representing $0-63 \%$ of the initial nitrate (Table 2.4 ). 
In contrast, when both nitrate and hydrogen were added to the incubations (Fig 2-2e, f), the total amount of ammonium produced was not only consistent between replicates, but also of a much greater magnitude than with either amendment alone. While ammonium was produced in both incubations at $24^{\circ} \mathrm{C}$ and $50{ }^{\circ} \mathrm{C}$, the increase in cell counts was more consistent and ammonium production more pronounced at $50^{\circ} \mathrm{C}$, supporting previous assertions that DNRA is a more important respiratory pathway at higher temperatures (Sievert and Vetriani, 2012). Despite the more rapid and greater extent of ammonium production at $50{ }^{\circ} \mathrm{C}$, the maximum amount of respired nitrate converted to ammonium was never greater than $55 \%$ (Table 2.4). Although we did observe evidence for ammonium assimilation during aerobic growth (Fig 2-2d), similar levels of assimilation are unlikely to have accounted for more than $15 \mu \mathrm{M}$ of the $\approx 45-65 \mu \mathrm{M}$ discrepancy. This suggests that denitrification to $\mathrm{N}_{2}$ or other $\mathrm{N}$ intermediates was still occurring at a significant level at elevated temperatures. Indeed, denitrification has been reported for the moderate thermophilic epsilonproteobacterium Nitratiruptor tergarcus (Nakagawa et al., 2005c, temperature optimum $=55^{\circ} \mathrm{C}$ ).

Another important factor that may have constrained the proportion of nitrate converted to ammonium in our incubations is the type of electron donor. DNRA is typically supported by the oxidation of $\mathrm{H}_{2}$, whereas denitrification can occur with either hydrogen or reduced sulfur (Sievert and Vetriani, 2012, and references therein). Since we observed both sulfide oxidation and hydrogen oxidation in the $50^{\circ} \mathrm{C}$ incubation, it may be that the ammonium produced was directly coupled to hydrogen oxidation, whereas the sulfide consumption was coupled to denitrification. Indeed, the proportion of nitrate converted to ammonium closely mirrors the hydrogen consumed on an electron-equivalent level, while the remainder of nitrate loss not coupled to ammonium production can be sufficiently accounted for by sulfide consumption. Overall, these data suggest that the elevated ammonium concentrations observed in Crab Spa vent fluids may be the product of bacteria carrying out DNRA in the subseafloor and that hydrogen availability is an important factor determining whether or not ammonium is produced as a product of nitrate respiration in natural fluids.

\subsubsection{Rates of Chemosynthetic Processes at in situ Pressure and Tem- perature:}

Rate measurements provide an important constraint on biological processes at vents. Previous studies incubating vent fluids at both in situ temperature and pressure are, to our 
knowledge, limited to an experiment conducted by Wirsen et al. (1986). Therefore, this study greatly expands the range of data and can be used for comparison of rates to recent studies that were not carried out at in situ pressure (Bourbonnais et al., 2012; Perner et al., 2010, 2011, 2013).

In our study, rates were derived for sulfide consumption/production, hydrogen consumption, nitrate consumption, oxygen consumption and ammonia production where at least two time points showed a consistent and measurable change in chemical concentrations. These data are presented as both cell-specific rates (Table 2.4) and as absolute rates (Supplemental spreadsheet). In general, per-cell sulfide consumption rates were consistent across all measured conditions, whereas nitrate consumption rates were more variable and greatly enhanced by the presence of dissolved hydrogen, particularly at $50^{\circ} \mathrm{C}$. Hydrogen oxidation rates were similarly variable, with the lowest values found where no electron acceptor was added (hydrogen-only amendment) and the highest rates found where nitrate was also added, with incubations at $50^{\circ} \mathrm{C}$ further enhancing rates. Higher rates of ammonium production were correlated with faster rates of hydrogen oxidation, while sulfide production was only seen in the hydrogen-only treatment. Since oxygen was only measured in four cases, it is difficult to make specific conclusions regarding the rate of its consumption. Below, we discuss how these rates compare to other studies conducted on diffuse-flow vent fluids.

Similar to the present study, Perner et al. (2013) performed amendments of deep-sea vent fluids collected at various sites on the Mid-Atlantic Ridge and compared results to unamended samples. There are significant differences in rates between their study and ours, especially for absolute sulfide oxidation rates which were approximately half of those reported here. However, normalization of rates to total cell numbers yielded rates that are comparable. For example, sulfide consumption rates of $239-797 \mathrm{fmol}_{2} \mathrm{~S}$ cell $^{-1}$ day $^{-1}$ in the present study (Table 2.4) are consistent with the range of 1.7-432 $\mathrm{fmol} \mathrm{H}_{2} \mathrm{~S}_{\text {cell }}{ }^{-1}$ day $^{-1}$ reported by Perner et al. (2013) for sulfide addition experiments. Cell count normalized hydrogen oxidation rates in the range of $0.5-2208 \mathrm{fmol} \mathrm{H}_{2}$ cell $^{-1}$ day $^{-1}$ determined by Perner et al. (2013) are similar to values of 49.6-1535 $\mathrm{fmol} \mathrm{H}_{2}$ cell $^{-1}$ day $^{-1}$ determined during this study (Table 2.4), although their slowest rates were approximately 2 orders of magnitude lower than ours. The reason for this discrepancy is difficult to ascertain, but Perner et al. (2011) note large changes in community composition relative to natural communities, which is an indicator that physicochemical conditions during their incubations changed apprecia- 
bly from those found in situ. Such a change could indicate that not all microbes quantified in their incubations were active, possibly explaining lower per-cell rates. In contrast, our study saw only small changes in community composition, with the already-dominant $E p$ silonproteobacteria becoming more abundant as a result of incubations (Table 2.3).

Rates of nitrate consumption have also been determined in a variety of low temperature vent fluids from the Juan de Fuca Ridge by Bourbonnais et al. (2012) using isotope-labeled nitrogen additions to trace nitrate removal processes. In their study, denitrification rates ranged from $\approx 0.002-77 \mathrm{fmol} \mathrm{NO}_{3}^{-}$cell $^{-1}$ day $^{-1}$ (Easter Island and Hermosa vents, respectively) which represents a much larger range than in our study (58-655 $\mathrm{fmol} \mathrm{NO}_{3}^{-} \mathrm{cell}^{-1}$ day $^{-1}$, Table 2.4). While their highest denitrification rates compare well with our nitrate-only additions, a number of their incubations reported orders of magnitude lower denitrification rates compared to our study. It is unclear why there is such a large difference, since the cell densities in the source fluids used by Bourbonnais et al. (2012) are in the same range as the present study and were also mostly dominated by Epsilonproteobacteria. One key difference between studies is the concentration of nitrate amendments; in the present study, we used concentrations approximately ten-fold higher. Since Bourbonnais et al. (2012) show that natural nitrate deficits were negatively correlated with denitrification rates, this could imply that samples with large nitrate deficits may have had lower abundances of denitrifiers or that cells present were not expressing denitrification enzymes. Since our study site has similar nitrate deficits (Table 2.2), yet we observed high per-cell denitrification rates (Table 2.4 ), it could be that higher concentrations of amendments permitted the reactivation of these pathways or the growth of denitrifiers. It is also possible that either decompression or experimental manipulation of fluids (or a combination of these factors) accounts for the large range of values observed in their study. Bourbonnais et al. (2012) report that fluids were purged with helium in some cases prior to incubation, and were allowed to remain at room temperature for $12 \mathrm{~h}$ prior to measurements in other cases - both of which could affect rate measurements. Purging removes volatile redox-active species such as sulfide, hydrogen, and methane, and since our results show that holding samples for $12 \mathrm{~h}$ in sealed vessels will deplete all available electron acceptors, not just oxygen (Fig 2-2a, b), microbes in either of these situations would have been deprived of energy sources for growth. As a result, they may have died or become less active, possibly accounting for some of the lower rates. Moreover, if most autotrophic energy sources were removed due to purging or consump- 
tion, the rates reported by these authors may represent background levels of heterotrophic denitrification instead of autotrophic processes.

Bourbonnais et al. (2012) also showed that denitrification was the dominant nitrate removal process, with rates exceeding those of DNRA by up to 160-fold. In the presence of hydrogen, the present study demonstrates significantly higher rates of DNRA and that the proportion of $\mathrm{NO}_{3}{ }^{-}$respired via DNRA was only 3 to 9 -fold lower than denitrification. Since dissolved hydrogen is thought to be the main electron donor for DNRA (discussion above; Sievert and Vetriani, 2012, and references therein), experimental approaches that provide hydrogen as an amendment may represent a more accurate constraint on potential DNRA rates in the natural environment.

Total nitrogen loss in the subseafloor biosphere was also calculated by Bourbonnais et al. (2012) by multiplying denitrification rates determined in their study with fluid flux and estimated residence time of the fluids. If the rates of denitrification determined in our study are used as input for this calculation, it would make nitrate removal processes by the global deep-sea vent subseafloor microbial community a much more significant sink in the global N-cycle, bringing it into the range of benthic denitrification (Bourbonnais et al., 2012; Gruber, 2004; Codispoti, 2007). While this suggests that denitrification at vents may have been underestimated, there are several important caveats. As discussed above, oxygen was more rapidly consumed than nitrate in our incubations, and yielded a higher number of cells (Fig 2-2). Therefore, it is likely that in natural environments oxygen is the preferred electron acceptor, and that denitrification will be suppressed where oxygen is non-limiting, although there may be a threshold below which so-called "aerobic denitrification" occurs (Gao et al., 2009), Fig 2-2d and supplemental spreadsheet. In addition, the residence time of fluids within a subsurface biosphere and the mixing ratio between seawater and vent fluid should ultimately control oxygen concentrations in low temperature hydrothermal vent fluids, and therefore denitrification rates. Higher proportions of seawater will introduce more oxygen and shorter residence times will reduce the opportunity for resident microbes to completely consume oxygen present. Although the mixing ratio can be well-constrained based on fluid composition, residence time and the extent of "aerobic denitrification" are relatively unknown, demonstrating a need for more research to accurately constrain nitrogen loss in the subseafloor biosphere. 


\subsection{Summary:}

In the present study, novel methods were developed that allowed IGT samplers to be used for incubations of deep-sea hydrothermal vent microbial communities under simulated seafloor conditions. IGT samplers were modified to allow both initial amendments and subsamples during short, $\approx 24 \mathrm{~h}$ incubations, permitting time-series measurements of chemical concentrations. Results from eighteen incubations, representing seven separate treatments in biological replicates, provide the basis for future biogeochemical models and reveal important gaps in our understanding of microbially-catalyzed processes at deep-sea hydrothermal vents. Data generated in this study have confirmed predicted microbial processes, quantified their rates, and revealed insights into the stoichiometry of metabolically relevant redox reactions. In addition, because incubations were short ( $\leq 1$ day) and amendment concentrations low $(\approx 100 \mu \mathrm{M})$, the microbial community composition was minimally altered from background communities. Therefore, the results reported here may be representative of rates in the natural environment and can be further combined with vent fluid chemistry to constrain hydrothermal vent microbial communities' contributions to biogeochemical cycling. Finally, since these methods can be applied to any pelagic deep-sea microbial community, incubations conducted in IGT samplers have great potential to increase our understanding of microbial processes and their quantitative importance in a broad range of deep-sea environments.

\subsection{Acknowledgements:}

We thank the officers, crew, and pilots of the R/V Atlantis and ROV Jason for their expert help at sea and their outstanding efforts acquiring the samples needed for this study. We also thank the scientific party for support, with special thanks going to Kerry McCulloch, Miriam Sollich, and Xi Wei for invaluable help with shipboard lab experiments. Many thanks are also due to Ben van Mooy for lending his lab's oxygen optode system, to Scott Wankel, Carly Buchwald and Zoe Sandwith for help quantifying nitrate/nitrite after the cruise, to Niculina Musat for assistance with CARD-FISH, to Virginia Edgcomb for lending her lab's Aanderaa in situ oxygen optode, and to Jeremy Rich for providing nitrate measurements for background seawater.

This research was funded by a grant of the Dimensions of Biodiversity program of the 
National Science Foundation to S.M.S, J.S.S., and C.D.T (NSF-OCE-1136727). Funding for J.M. was provided by doctoral fellowships from the National Sciences and Engineering Research Council of Canada (NSERC-PGSD3) and the National Aeronautics and Space Administration Earth Systems Science Fellowship (NESSF), an award from the Canadian Meteorological and Oceanographic Society, as well as funding from WHOI academic programs and the aforementioned National Science Foundation grant. 
Chapter 3

Physiologically Diverse

Epsilonproteobacteria Dominate

Subseafloor Autotrophy at a

Deep-Sea Hydrothermal Vent 


\subsection{Abstract:}

Chemoautotrophic microbial communities that inhabit the deep-sea subseafloor biosphere at hydrothermal vents influence oceanic biogeochemistry and provide new carbon to the deep sea, yet constraining their activity in situ remains challenging. Here, we report microbial activity measurements from subsurface-derived hydrothermal fluids incubated with multiple amendments at in situ pressure/temperature. Using taxonomically-resolved single-cell mass spectrometry, we identified Epsilonproteobacteria as the dominant active chemoautotrophs under all conditions. Although Epsilonproteobacteria were consistently dominant, amendments markedly affected community structure, revealing factors that both influence niche differentiation and physiological diversity within subseafloor communities. By normalizing carbon fixed to electron acceptors consumed, we derived community chemosynthetic growth

efficiency. In turn, this key parameter was used to constrain subseafloor carbon fixation and biomass standing stock. Overall, our data constrain in situ primary productivity and show how physicochemistry may influence population structure and function in the subseafloor biosphere at deep-sea vents. 


\subsection{Introduction:}

Almost forty years ago, the discovery of deep-sea hydrothermal vents revealed that microbes support entire ecosystems via chemosynthetic carbon fixation (Karl et al., 1980). While the taxonomy and metabolic potential of such microbes is now better known (Huber et al., 2007; Nakagawa and Takai, 2008), much less is known about their metabolic activity in situ. In the hydrothermal vent subseafloor biosphere, hot reducing hydrothermal fluid and cold oxidizing seawater mix in the ocean crust, creating conditions conducive for chemosynthetic microorganisms. However, this habitat remains very poorly characterized. Although the subseafloor is enriched in biomass (Karl et al., 1980) and hosts active carbon fixation (Wirsen et al., 1986, 1993; Perner et al., 2013), our understanding of subseafloor primary productivity and its contribution to biogeochemical cycles remains poorly constrained. This is for two main reasons. Firstly, experiments carried out at atmospheric pressure (Perner et al., 2013) may be influenced by decompression of fluids and/or microbes. Secondly, even for those few studies conducted under in situ conditions (Wirsen et al., 1986, 1993) only carbon fixation has been quantified - not the specific redox reactions used by microbes to generate energy. Furthermore, which subseafloor microorganisms actively contribute to chemoautotrophy and how efficiently they convert available energy into biomass is unknown. However, without such data, the productivity of the biosphere in areas of hydrothermal activity and its impact on oceanic biogeochemical cycling remain difficult to estimate.

In this study, we addressed these limitations by incubating subseafloor microbial communities with nitrate, oxygen, and hydrogen amendments (which are depleted by microbial activity upon sampling (McNichol et al., 2016)) as well as a ${ }^{13} \mathrm{CO}_{2}$ label (to track carbon fixation). Microbes were maintained at in situ pressure and temperature for $\approx 24 \mathrm{~h}$ using isobaric gas-tight samplers (25MPa; McNichol et al., 2016; Seewald et al., 2002). Most ex-

periments were carried out at in situ vent fluid temperature $\left(24^{\circ} \mathrm{C}\right)$, while an additional two were incubated at higher temperature $\left(50^{\circ} \mathrm{C}\right)$ that likely characterizes deeper subseafloor environments.

\subsection{Materials and Methods:}

Fluid samples for all analyses were collected with the ROV Jason II aboard the $\mathrm{R} / \mathrm{V}$ Atlantis during research cruise AT26-10 in January 2014 from Crab Spa, which is located at a 
depth of $2506 \mathrm{~m}$ at $9^{\circ} 50.3981 \mathrm{~N}, 104^{\circ} 17.4942 \mathrm{~W}$. Shipboard incubations of fluids were carried out at in situ pressure for $\approx 18-24 \mathrm{~h}$ with amendments of $\mathrm{H}_{2}, \mathrm{NO}_{3}{ }^{-}, \mathrm{O}_{2}$ and $\mathrm{H}_{2} / \mathrm{NO}_{3}{ }^{-}$. With the exception of $2 \mathrm{NO}_{3}{ }^{-} / \mathrm{H}_{2}$ incubations carried out at $50{ }^{\circ} \mathrm{C}$, all other incubations were conducted at $24^{\circ} \mathrm{C}$, which is the in situ fluid temperature at Crab Spa. During incubations, cell abundance and concentrations of selected chemical species $\left(\mathrm{H}_{2} \mathrm{~S}, \mathrm{H}_{2}, \mathrm{NO}_{3}{ }^{-}, \mathrm{NH}_{4}{ }^{+}\right.$and $\mathrm{O}_{2}$ ) were measured every $\approx 6 \mathrm{~h}$. Full details of sampling, incubation procedures, chemical measurements, cell counts and rate measurements are described in McNichol et al. (2016).

\subsubsection{DNA Analyses:}

At the end time point of each experiment $(\approx 24 \mathrm{~h})$, the remaining volume $(\approx 8-40 \mathrm{~mL})$ was drawn into a clean, sterile and DNA-free syringe (Norm-Ject), filtered through a $0.2 \mu \mathrm{M}$ Sterivex filter cartridge, dried under filtered nitrogen gas and frozen immediately at $-80^{\circ} \mathrm{C}$. DNA was extracted as previously described (Signori et al., 2014). Briefly, filter pieces containing cells were lysed in a $\mathrm{NaCl}$-sucrose buffer with lysozyme, SDS and proteinase $\mathrm{K}$, and DNA was subsequently precipitated with sodium acetate/ethanol and linear acrylamide as a co-precipitant due to low concentrations of DNA present. DNA extraction success was verified using the bacterial primers 27Fmod and 519Rmodbio, and amplicons were subsequently sequenced using the same primers and 454-pyrosequencing technology (Molecular Research LP, Shallowater TX, USA).

Pyrotags sequences were analyzed using the QIIME pipeline (Caporaso et al., 2010). Initially, sequences were quality filtered with split_libraries.py (-w 50 -r -1 300 -L 1000 -a 0 -H 6 -b 8 -z truncate_only), then denoised from 454 flowgrams (denoise_wrapper.py). After denoising, chimeras were removed using the script "identify_chimeric_seqs.py" with USEARCH (v6.1) as the method. This yielded $3597 \pm 1371$ (standard deviation) sequences per sample. 97\% OTUs were picked de novo using the script pick_otus.py, with USEARCH (v6.1) as the method and classified with the script assign_taxonomy.py using the SILVA v119 database as a reference and BLAST as the method. It was noted that some OTUs affiliated with Sulfurimonas were incorrectly identified as the taxon "BR36"; the taxonomy of these OTUs were manually inspected and changed. Raw sequences in .sff format are deposited at NCBI under accession number SRP077942.

Correlations of community composition with environmental parameters was carried out with a subset of total sequences. Sulfurimonas $97 \%$ OTUs found in $24^{\circ} \mathrm{C}$ incubations 
were first manually normalized within each sample as the percentages of total Sulfurimonas sequences. Next, beta diversity was calculated using beta_diversity.py with UniFrac as the distance metric. A tree of sequences necessary for the UniFrac metric was generated by aligning sequences using MUSCLE (align_seqs.py) and building a tree using default parameters (make_phylogeny.py). The divergence between these different communities of Sulfurimonas was visualized by using the script nmds.py to generate values for a 2-D Nonmetric Multidimensional Scaling plot. Next, the script compare_categories.py was used with the adonis method to investigate the effect of the following variables (at the beginning of incubations) on final Sulfurimonas OTU composition: $\mathrm{pH},\left[\mathrm{H}_{2}\right],\left[\mathrm{H}_{2} \mathrm{~S}\right],\left[\mathrm{NH}_{3}\right],\left[\mathrm{NO}_{3}{ }^{-}\right], \mathrm{pO}_{2},\left[\mathrm{CH}_{4}\right]$, cell density and time from seafloor until the beginning of incubations. Finally, the script observation_metadata_correlation.py with pearson correlations was used to look for the effect of $\mathrm{pO}_{2}$ on individual Sulfurimonas OTUs.

\subsubsection{CARD-FISH $/{ }^{13} \mathrm{C}$ incorporation:}

Aliquots of approximately $10 \mathrm{~mL}$ of culture fluid were preserved at 16 or $24 \mathrm{~h}$ after the addition of labels/amendments with paraformaldehyde (1\%, $1 \mathrm{hr}$ at room temperature). Cells were then filtered under moderate vacuum onto $\mathrm{Au} / \mathrm{Pd}$-sputtered $0.2 \mu \mathrm{M}$ polycarbonate filters, washed $2 \mathrm{x}$ with $10 \mathrm{~mL} 1 \mathrm{x}$ PBS, air-dried and stored at $-20^{\circ} \mathrm{C}$ prior to further analysis.

Filters were embedded in low-melting point agarose, endogenous peroxidases were inactivated by immersion in $3 \% \mathrm{H}_{2} \mathrm{O}_{2}$ for $10 \mathrm{~min}$, and permeabilized for $30 \mathrm{~min}$ at $37^{\circ} \mathrm{C}$ in a $10 \mathrm{mg} \mathrm{mL} \mathrm{m}^{-1}$ solution of lysozyme in TE buffer. Hybridization and tyramide amplification were conducted at $46^{\circ} \mathrm{C}$ for $3 \mathrm{~h}$ and $20 \mathrm{~min}$, respectively. Oregon Green $488-\mathrm{X}$ was used for tyramide amplification, which contains two atoms of fluorine per molecule. All newly designed probes and their formamide concentrations are shown in Table A.2. Newly designed

probes were tested with positive and negative control cultures across a melting curve to determine both the potential for non-specific hybridization and the optimum concentration of formamide (Table A.2). Probes were additionally tested to insure specificity by doing a double hybridization with both the EPSI549-914 combination and the newly designed probes on natural environmental samples (where other organisms aside from Epsilonproteobacteria were present). Since cells hybridized with NAUT921 and SFMN287 were also hybridized with the EPSI549-914 probe, this was additional confirmation that these probes are specific 
to Epsilonproteobacteria.

Once hybridized, $5 \mathrm{~mm}$ diameter circular sections were cut out from each filter, and regions of interest were marked with a laser-dissecting fluorescence microscope with a $63 / \mathrm{X}$ air objective. The remaining portions of filters were used to count the percentage of DAPIstained cells hybridized to each specific probe. Seven grids were analyzed per sample, amounting to 400-700 DAPI-stained cells.

Regions of interest or random grids hybridized with the EPSI549-914 probes were analyzed on NanoSIMS 50L Ionprobe from CAMECA (AMETEK), detecting the following ions: ${ }^{12} \mathrm{C},{ }^{13} \mathrm{C},{ }^{12} \mathrm{C}^{14} \mathrm{~N},{ }^{13} \mathrm{C}^{14} \mathrm{~N},{ }^{19} \mathrm{~F}, \mathrm{Au},{ }^{32} \mathrm{~S}$ and ${ }^{34} \mathrm{P}$. An average of 49.6 target cells were analyzed per IGT incubation for the EPSI probes, with a range of 22-96. A subset of three samples were also analyzed with the NAUT921 probe (between 14-21 cells per sample).

\subsection{3 ${ }^{13} \mathrm{C}$ isotope incorporation into bulk biomass:}

At the last time point during experiments, a known volume of culture fluid $(\approx 20 \mathrm{~mL})$ was filtered onto a pre-combusted GF-75 glass fiber filter ( $0.3 \mu \mathrm{M}$ pore size; Advantec), wrapped in combusted aluminum foil, and stored at $-80^{\circ} \mathrm{C}$ prior to further analysis.

Filters were acidified to remove carbonates by exposure to $\mathrm{HCl}$ vapor for 3 days at 60 - $65 \mathrm{C}$, then dried for 1 day at the same temperature. Immediately prior to combustion, dried filters were wrapped with tin foil (Costech part \# 041073) and folded into pellets. Samples were combusted in a Carlo Erba / Fisons 1107 Elemental Analyzer "EA" (fitted with a Costech "Zero-Blank" carousel). The EA is attached via Finnigan-MAT Conflo-II interface to a DeltaPlus stable isotope ratio mass spectrometer. Data were acquired using the Isodat (version 2.5) software.

Carbon fixation rate determinations: For all incubations, ${ }^{13} \mathrm{C}$ DIC was supplied as a ${ }^{13} \mathrm{HCO}_{3}{ }^{-}$solution dissolved in filtered bottom seawater and added into low-temperature hydrothermal fluid (McNichol et al., 2016). The fraction of total DIC as ${ }^{13} \mathrm{C}$ label was determined using measured [DIC] values for background seawater and vent fluid, and was approximately $10 \%$ in all cases. A conversion factor derived from these label percentages was used in both rate determinations below to derive total $\mathrm{CO}_{2}$ fixation rates.

For rate determinations from bulk isotope incorporation measurements, background

${ }^{13} \mathrm{C}$ from an average of background (unincubated) samples was subtracted from detected ${ }^{13} \mathrm{C}$ and normalized as described above to determine total $\mathrm{CO}_{2}$ fixed. Rates were determined by 
dividing total carbon fixed by the time from label addition to when samples were taken.

For HISH-SIMS derived rates, data was processed with Look@NanoSIMS (Polerecky et al., 2012) to demarcate regions of interest for EPSI549/914-hybridized cells based on the

${ }^{19} \mathrm{~F}$ signal. Cell biovolume was estimated using the area and length:width ratio parameters for each region of interest, which was then combined with cell carbon density previously reported (Lee and Fuhrman, 1987) to estimate carbon content for each cell. The amount of $\mathrm{CO}_{2}$ fixed per cell was then determined by correcting ${ }^{13} \mathrm{C}^{14} \mathrm{~N} /{ }^{12} \mathrm{C}^{14} \mathrm{~N}$ ratios for background ${ }^{13} \mathrm{C}$ and label concentrations in fluids. This value was then multiplied by EPSI-hybridized cells $\mathrm{mL}^{-1}$ and normalized by time to yield total $\mathrm{CO}_{2}$ fixed per volume per time.

\subsubsection{Chemosynthetic growth efficiency (CGE) determinations:}

CGE is the estimated fraction of electron equivalents derived from electron donors that are used to reduce $\mathrm{CO}_{2}$ into biomass, equivalent to the parameter 1-y from (Klatt and Polerecky, 2015). Total carbon fixed from bulk isotope measurements was determined as described above. The consumption of nitrate and oxygen were also measured - likely the only electron acceptors of importance during incubations. The means by which electron equivalents used to reduce these substrates was calculated has been previously described (McNichol et al., 2016). Total electrons oxidized from sulfide and hydrogen were not directly measurable due to incomplete oxidation of sulfide (McNichol et al., 2016), so this value was inferred by taking the sum of electron equivalents to carbon fixation and electron equivalents to electron acceptors. CGE was then derived by dividing total carbon fixed by this sum.

\subsubsection{Extrapolations to natural environment:}

In situ primary productivity at our study site was estimated using two pieces of information. Firstly, depletions in electron acceptors associated with chemosynthesis (nitrate and oxygen) were used to determine the fingerprint of microbial activity in the natural environment (McNichol et al., 2016). Then, given the range of values for CGE, we estimated how much carbon could be fixed by microbes that had consumed these substrates during active growth. This generated values of carbon fixed per volume of mixed low-temperature hydrothermal vent fluid. These values were normalized per volume end-member fluid to compare with values from (McCollom and Shock, 1997). Normalization was accomplished by considering the fraction of end-member fluid in Crab Spa mixed fluids. 
Standing stock was constrained by considering the minimum number of cells needed to account for the observed chemical depletions in situ using per-cell chemical consumption rates determined previously. Converting cell numbers to biomass therefore relied on an estimation of cell carbon density per volume. The conversion factor we used to convert NanoSIMS isotope ratios to per-cell carbon fixation rates (described above) was determined for small, pelagic cells (Lee and Fuhrman, 1987). Since the ratio between NanoSIMS and bulk isotope uptake rate measurements is similar to previously determined values (Musat et al., 2014), it suggests this was an appropriate conversion factor for our experimental incubations. This is despite large differences in predicted biovolume $\left(0.8 \mu^{3}\right.$ vs. $0.04-0.08$ $\mu^{3}$ in (Lee and Fuhrman, 1987)).

For the natural environment, however, microscopic evidence suggests that cells are much larger on average $\left(\approx 2.5 \mathrm{\mu m}^{3}\right)$ and may weigh on the order of $600-700 \mathrm{fg}$ per cell. But since we only have information about the activity of smaller and not larger cells in the natural environment, we chose to use conservative values for cell size $\left(0.8 \mathrm{\mu m}^{3}\right)$ and a biovolume:cell carbon conversion factor more appropriate for larger cells (Loferer-KröSSbacher et al., 1998). This yielded a value of $173 \mathrm{fg} \mathrm{C}$ per cell, which can be considered a conservative minimum value for cell carbon content in the natural environment at Crab Spa. This value was then multiplied by the number of cells responsible for chemical depletions to determine the minimum standing stock. Residence time of biomass was then simply determined by assuming that primary productivity and export production were equivalent and solving for residence time.

The conservative value of $173 \mathrm{fg} \mathrm{C}$ per cell was also used to determine the amount of cell carbon present in natural fluids. Combined with dissolved organic carbon enrichments, these values allowed us to compare primary productivity estimates with enrichments of carbon found in situ.

In order to extrapolate these data to a larger scale, we assumed that volumetric primary productivity and standing stock for other low-temperature subseafloor ecosystems is the same as our study site. Then, by using published estimates of total fluid fluxes through lowtemperature vent systems, we could estimate primary productivity on a local and global scale. 


\subsection{Results and Discussion:}

Despite a diverse range of chemical amendments, our incubations were completely dominated by one group of Bacteria. 16S rRNA gene amplicon analysis (Fig 3-1a) showed Epsilonproteobacteria comprised the vast majority of sequences ( $\bar{x}=97.0 \pm 3.7 \%$ ), almost identical to catalyzed-reporter deposition fluorescence in-situ hybridization (CARD-FISH) counts (Table A.1, $\bar{x}=94 \pm 11 \%$ of total cells; McNichol et al. (2016)). Sequences were related to known chemoautotrophs, suggesting active $\mathrm{CO}_{2}$ fixation. This was confirmed by measuring ${ }^{13} \mathrm{CO}_{2}$ tracer incorporation in single epsilonproteobacterial cells with halogen in situ hybridization nanoscale secondary ion mass spectrometry (HISH-SIMS; Musat et al., 2008). HISH-SIMS demonstrated that amendments, in particular oxygen or a combination of nitrate and hydrogen, increased relative $\mathrm{CO}_{2}$ fixation rates (Fig 3-2a). Given that Epsilonproteobacteria dominate natural communities $(\approx 80 \%$ of total cells) and are the vast majority of microbes found in the incubations (Fig 3-1a; McNichol et al. (2016)), we conclude that Epsilonproteobacteria dominate subseafloor primary production at our study site. 


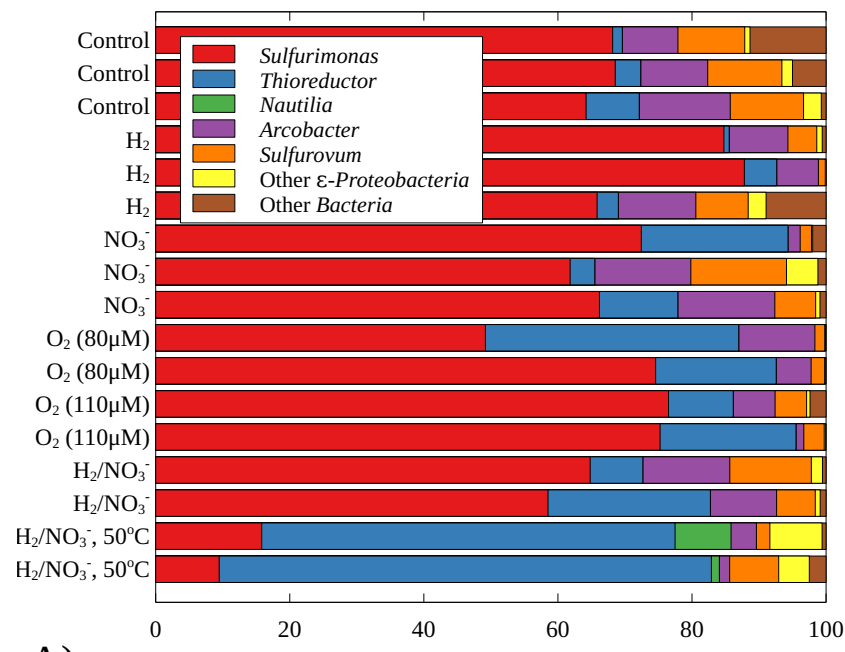

A)
B)

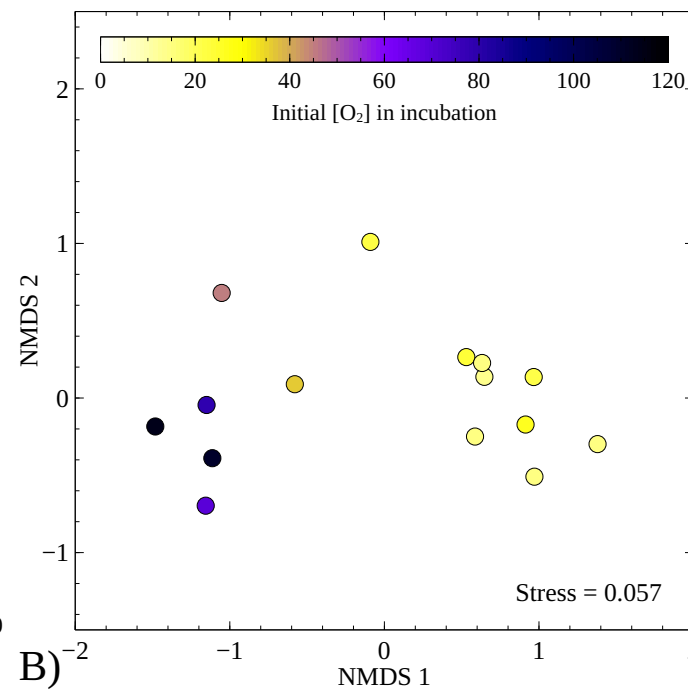

NMDS 1
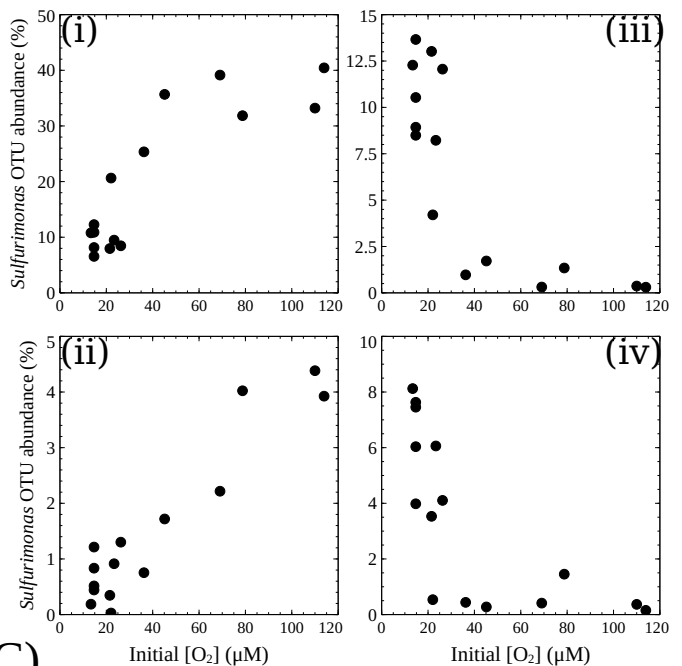

C)

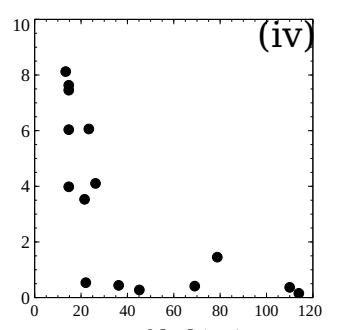

Initial $\left[\mathrm{O}_{2}\right](\mu \mathrm{M})$

Figure 3-1: Bacterial community composition during incubations. (A) Genus-level composition, (B) Nonmetric multidimensional scaling plot showing divergence in Sulfurimonas $97 \%$ operational taxonomic unit (OTU) composition overlaid with initial $\mathrm{pO}_{2}$ for $24{ }^{\circ} \mathrm{C}$ incubations and (C) selected correlations between the abundance of these Sulfurimonas OTUs and initial $\mathrm{pO}_{2}$. All sequences in (A) are Epsilonproteobacteria except for "Other Bacteria". 
We also quantified ${ }^{13} \mathrm{CO}_{2}$ tracer incorporation into total microbial biomass (Musat et al., 2008) which is unbiased by losses of tracer that occur during preparations for HISHSIMS (Musat et al., 2014). Consistent with previous results, these measurements were $\approx 45 \%$ higher than those derived from HISH-SIMS. Therefore, these data are better suited to determine absolute rates of carbon fixation. Rates were consistently high $(\approx 50-200$ $\mu \mathrm{g} \mathrm{C} \mathrm{L} \mathrm{C}^{-1} \mathrm{~d}^{-1}$; Fig A-3), far exceeding most values reported elsewhere $(\approx 2-650 \mathrm{x}$ vs. (Wirsen et al., 1986, 1993); $\approx 900 \mathrm{x}$ on average vs. (Perner et al., 2013)). However, our rates are similar to Mandernack and Tebo (1999) who cite short incubation times ( $\leq$ to our study) as an explanation for higher rates. Therefore, the short timescale of incubations and maintenace of deep-sea pressure and temperature (McNichol et al., 2016) probably explains why carbon fixation rates reported here are consistently higher than most other studies.

Since the amount of carbon fixed during incubations clearly increased as a result of amendments (Fig 3-2a, Fig A-3), we normalized absolute carbon fixed by the amount of nitrate and oxygen consumed during incubations, allowing us to determine the chemosynthetic growth efficiency (CGE). CGE is the fraction of electrons from donors that are used to reduce $\mathrm{CO}_{2}$ :

$$
C G E=\frac{E q_{C F I X}}{\left(E q_{C F I X}+E q_{D I S S}\right)}
$$

Where EqCFIX = electron equivalents to carbon fixation (assuming biomass oxidation state $=0)$ and EqDiss $_{\text {D }}=$ electron equivalents to dissimilatory processes $\left(\mathrm{O}_{2}\right.$ reduction and $\mathrm{NO}_{3}{ }^{-}$reduction by denitrification or DNRA). Note: Denominator contains EqCFIx to infer total electrons oxidized, which was not directly measurable due to uncertainties in the product of sulfide oxidation (McNichol et al., 2016)

CGE provides an empirical measurement of the efficiency at which natural microbial communities convert $\mathrm{CO}_{2}$ into biomass under in situ conditions. In contrast, previous studies have used thermodynamic calculations to estimate total bioavailable energy and used this data to estimate primary productivity in situ. However, these studies have relied on assumptions to either convert energy to ATP and subsequently biomass (McCollom and Shock, 1997) or have used values for cellular maintenance energy to estimate total biomass standing stock (Nakamura and Takai, 2015). However, these assumptions are have not been rigorously tested with hydrothermal vent communities or isolates. Here, we avoid such assumptions by directly measuring CGE under deep-sea conditions. 
Measuring CGE also gives information about how incubation conditions affect the microbial community's overall efficiency of carbon fixation. For example, with nitrate or coupled nitrate/hydrogen amendments at $24^{\circ} \mathrm{C}$, CGE was close to pure cultures of mesophilic sulfur-oxidizing Epsilonproteobacteria (Fig 3-3; Klatt and Polerecky (2015)). However, with oxygen additions at $24^{\circ} \mathrm{C}$ and nitrate/hydrogen additions at $50{ }^{\circ} \mathrm{C}$, CGE decreased by approximately $50 \%$.

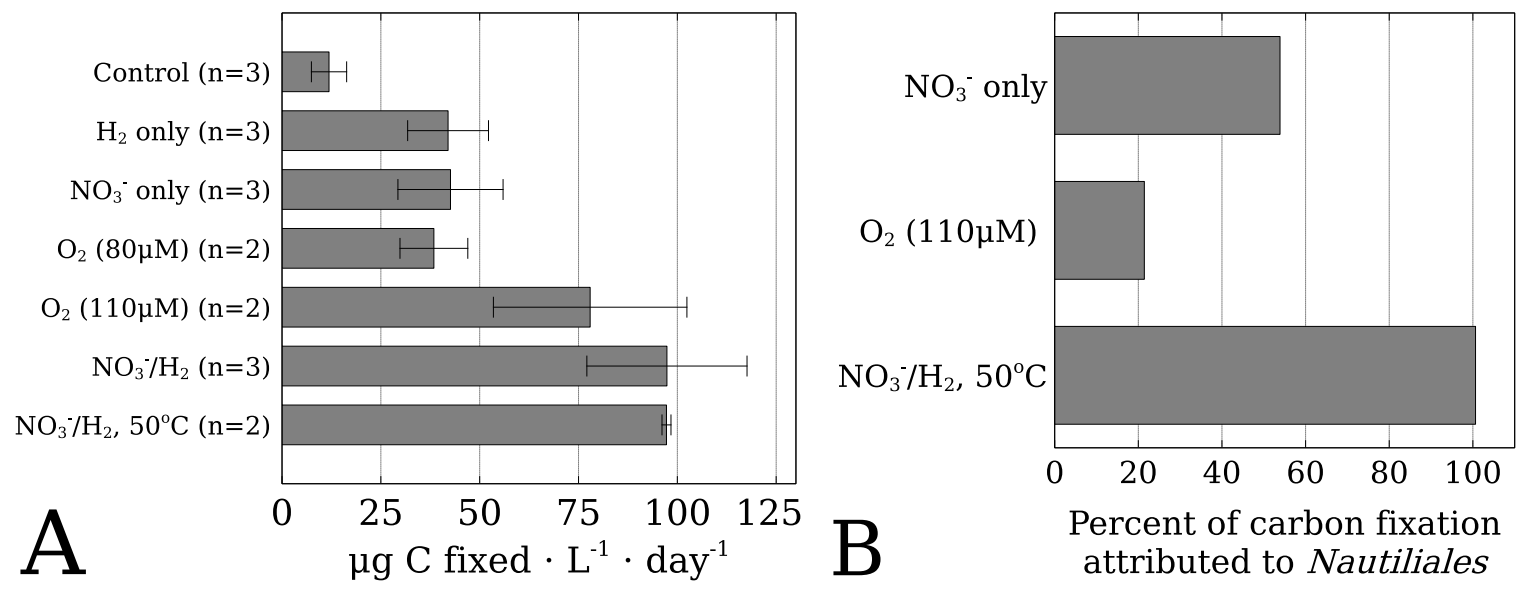

Figure 3-2: Relative estimations of primary productivity in incubations of hydrothermal vent fluids at in situ temperature and pressure determined by halogen in-situ hybridization secondary ion mass spectrometry (HISH-SIMS). (A) Relative volumetric rates of epsilonproteobacterial $\mathrm{CO}_{2}$ assimilation during incubations. (B) Proportion of productivity attributed to the order Nautiliales (Epsilonproteobacteria) in selected treatments. No errors are presented in (B) because percentages are derived from one biological replicate. Errors are standard deviations $(n=3)$, or ranges $(n=2)$. Values in $(A)$ are not corrected for the influence of CARD-FISH procedure (Musat et al., 2014).

Since variable CGE under these different conditions could be due to the selective enrichment of organisms with different physiologies, we looked for correlations between community composition and incubation conditions. Across all incubations at $24^{\circ} \mathrm{C}$, the genus Sulfurimonas dominated both amplicon sequences $(\bar{x}=68 \%$; Fig $3-1 \mathrm{a})$ and CARD-FISH counts $\left(\bar{x}=72 \%\right.$ of total cells; Table A.1). While uniformly dominant at the genus level, $\mathrm{pO}_{2}$ at the beginning of incubations had a statistically-significant influence on final Sulfurimonas OTU composition (adonis test, $\mathrm{R}^{2}=0.52 ; \mathrm{p}=0.001$; other parameters were not statistically significant Table A.3). This divergence in OTU composition (Fig 3-1B) was due to the positive/negative response of many individual Sulfurimonas $97 \%$ OTUs to $\mathrm{pO}_{2}$. Amongst the 24 most abundant Sulfurimonas OTUs (comprising at least $2 \%$ of Sulfurimonas sequences in at least one incubation), 16 possessed a statistically-significant correlation to $\mathrm{pO}_{2}$ (Ta- 


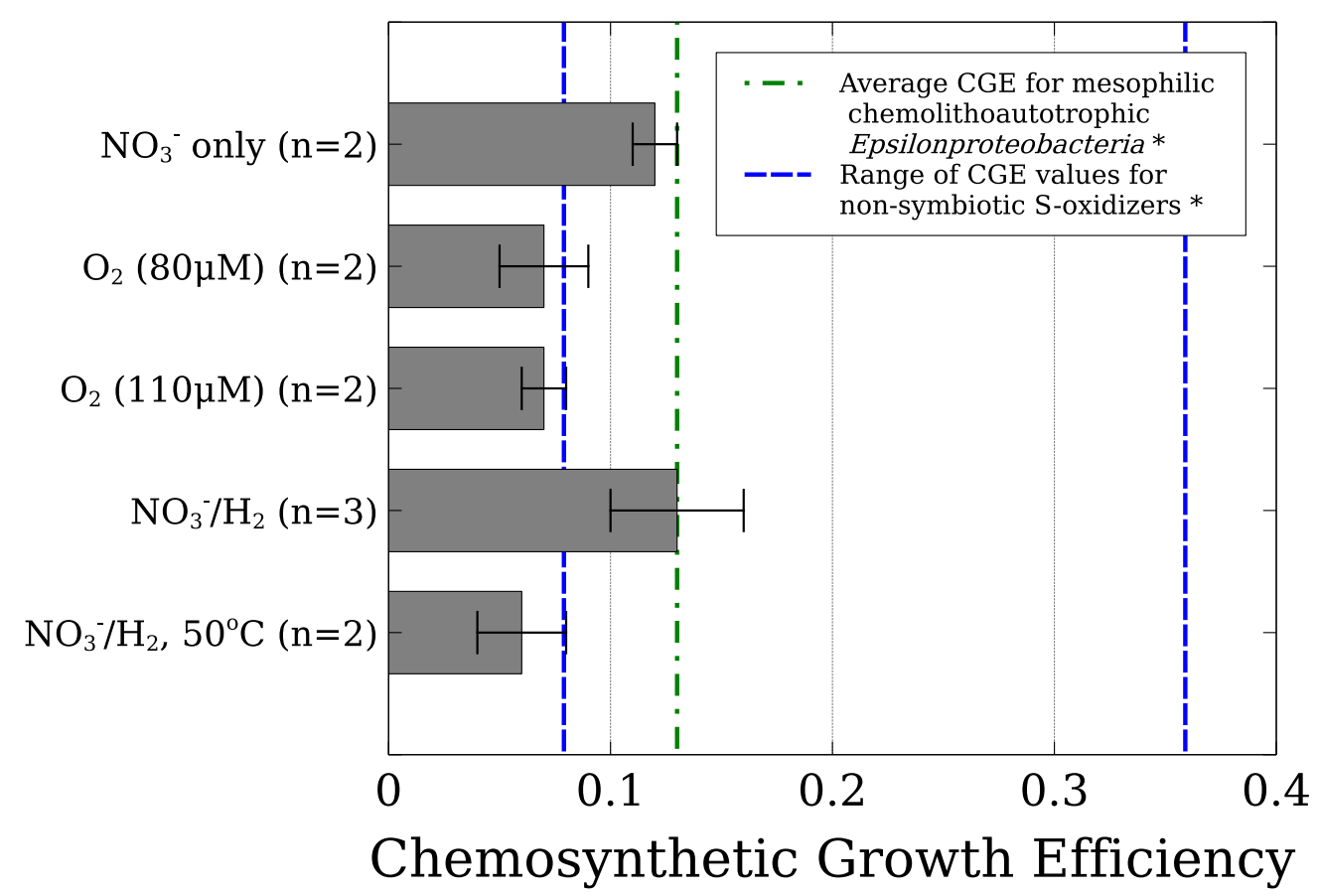

Figure 3-3: Fraction of electrons from electron donors used for carbon fixation (chemosynthetic growth efficiency). Values were determined from concomitant measurements of absolute carbon fixed and nitrate/oxygen consumed in high-pressure incubations under deep sea conditions. Errors are standard deviations $(\mathrm{n}=3)$, or ranges $(\mathrm{n}=2)$. * Values from (Klatt and Polerecky, 2015)

ble A.4). Since these correlations were both positive or negative, it strongly suggests these OTU-based clusters represent physiologically distinct organisms that are either $\mathrm{O}_{2}$-tolerant or -sensitive (Fig 3-1c).

On a community level, the organisms growing at high $\mathrm{pO}_{2}$ fixed smaller amounts of $\mathrm{CO}_{2}$ per unit electron donor relative to treatments without added $\mathrm{O}_{2}$ (Fig 3-3). This is counterintuitive, since bioenergetics predicts higher growth yields with $\mathrm{O}_{2}$ vs. nitrate (Chen and Strous, 2013). While slightly higher growth yields have been measured for one Sulfurimonas strain in aerobic vs. anaerobic conditions (Timmer-Ten Hoor, 1981), oxygen is also toxic to Sulfurimonas at variable and strain-specific levels (Timmer-Ten Hoor, 1981; Takai et al., 2006). This is likely due to $\mathrm{O}_{2}$ directly inactivating ferredoxin-dependent enzymes of the reverse tricarboxylic acid cycle (Imlay, 2006). Therefore, the apparently high $\mathrm{pO}_{2}$-tolerant Sulfurimonas OTUs (e.g. (i) and (ii) in Fig 3-1c) likely have biochemical adaptations to prevent enzyme inactivation. Since such adaptations could reduce growth yields (Berg, 2011), it may explain why organisms in our high $\mathrm{pO}_{2}$ treatments fix less $\mathrm{CO}_{2}$ 
into biomass per unit substrate oxidized.

We also observed lower $\mathrm{CGE}$ at $50^{\circ} \mathrm{C}$ where $16 \mathrm{~S}$ rRNA sequences $(\bar{x}=76 \%$; Fig 3-1a) and CARD-FISH counts ( $\bar{x}=84 \%$ of total cells; Table A.1) were dominated by the order Nautiliales - a deep-branching lineage within the Epsilonproteobacteria. It has been suggested previously that thermophiles have higher maintenance energy requirements compared to mesophiles (Zeikus, 1979).

Interestingly, Nautiliales cells likely belonging to the genus Thioreductor (Fig 3-1a) were also present in a variety of conditions at $24^{\circ} \mathrm{C}$ (up to $38 \%$ of sequences (Fig 3-1a) and $29 \%$ of total cells (Table A.1)). Thioreductor micantisoli - the sole cultivated representative of this genus - is dependent on hydrogen and an obligate anaerobe (Nakagawa et al., 2005a). Therefore, its presence without added hydrogen and with oxygen amendments up to $110 \mu \mathrm{M}$ was unexpected. To confirm these organisms were active, we used HISH-SIMS to measure ${ }^{13} \mathrm{CO}_{2}$ tracer incorporation into Nautiliales cells. At $50{ }^{\circ} \mathrm{C}$ with added hydrogen, Nautiliales cells (likely Thioreductor and Nautilia) were responsible for $\approx 100 \%$ of carbon fixed (Fig $3-2 b)$.

While this was unsurprising, our data also showed Nautiliales cells (likely Thioreductor; Fig 3-1a) were active at $24{ }^{\circ} \mathrm{C}$ with both oxygen and nitrate amendments, accounting for up to $53 \%$ of carbon fixed (Fig 3-2b). In addition, we observed dissimilatory nitrate reduction to ammonium (DNRA) that was correlated to the abundance of Nautiliales cells (Fig A-1). Because DNRA is not known to occur in other taxa identified in the incubations (Fig 3-1, (Nakagawa and Takai, 2008)), it suggests Thioreductor cells produced this ammonium. To date, hydrogen is the only known electron donor for autotrophic DNRA (Nakagawa and Takai, 2008). However, it was not present in all cases where ammonium production was observed during our incubations (McNichol et al., 2016). Therefore, Thioreductor representatives likely used other compounds (possibly reduced sulfur) as electron donors for DNRA, suggesting these uncultivated cells possess novel physiological characteristics that differ from the isolate. Supporting this, Thioreductor-related OTUs at $24^{\circ} \mathrm{C}$ were phylogenetically distinct from those found at $50^{\circ} \mathrm{C}$ that have physiological attributes more similar to the pure culture (Fig A-2).

Together, these data suggest that organisms active in our incubations are physiologicallydiverse and provide evidence for mechanisms contributing to niche differentiation in natural populations. Active organisms were also genetically distinct from isolates (92\% 16S simi- 
larity for Thioreductor and $\approx 93 \%$ for most Sulfurimonas sequences), demonstrating that subseafloor populations contain diverse microbes not well-represented by current cultivars.

Using data on depletions of chemosynthetic substrates in natural fluids that indicate microbial consumption (McNichol et al., 2016), we applied our values for CGE (0.06-0.13) to estimate primary productivity in the subseafloor. At our study site (Crab Spa; a low temperature hydrothermal vent $\approx 306 \mathrm{~cm}^{2}$ (McNichol et al., 2016; Germanovich et al., 2015), we estimate that $104-253 \mu \mathrm{g} \mathrm{C}$ could be produced per L of mixed fluid; this is consistent with enrichments of $\approx 157 \mu \mathrm{g} \mathrm{C} \mathrm{L}^{-1}$ estimated from average cell numbers and total DOC in Crab Spa fluids ${ }^{1}$. Considering that Crab Spa fluid is $\approx 93 \%$ seawater and $\approx$ 7\% hydrothermal end-member fluid (McNichol et al., 2016), $1.43 .5 \mathrm{mg} \mathrm{C} \mathrm{L}^{-1}$ can be fixed per L of end-member fluid. Surprisingly, this is only $\approx 4-10 \%$ of other estimates based on thermodynamic models (380 mg wet weight per L end-member fluid: McCollom and Shock, 1997). Since similar fluid chemistry was used for estimates in McCollom and Shock (1997), this discrepancy is likely due to the assumptions made to convert free energy into carbon fixed assumptions that are completely unnecessary here because of our direct measurement of CGE.

To estimate total primary productivity and maximum carbon export, we use a fluid flux of $1.87 \mathrm{~L} \mathrm{~s}^{-1}$ at Crab Spa (Germanovich et al., 2015) to calculate that $6.1 \times 10^{3}$ to $1.5 \times 10^{4} \mathrm{~g} \mathrm{y}^{-1}$ of carbon is produced at Crab Spa; this is orders of magnitude greater than photosynthetic biomass predicted to reach this depth $\left(0.4-4 \mathrm{~g} \mathrm{C} \mathrm{m}^{-2} \mathrm{y}^{-1}\right.$, (Lampitt and Antia, 1997)). With a total low-temperature fluid flux of the vent field at $9^{\circ} 50^{\prime} \mathrm{N}$ East Pacific Rise of $1170 \mathrm{~kg} \mathrm{~s}^{-1}$ (Lowell et al., 2012), we estimate a total vent field productivity of $3.8 \times 10^{6} 9.3 \times 10^{6} \mathrm{~g} \mathrm{C} \mathrm{y}^{-1}$. If this biomass remained in the vent field $\left(10^{3}-10^{4} \mathrm{~m}^{2}\right.$ (Lowell et al., 2012)), it would represent a very high concentration of labile carbon for the deep sea $\left(3.8 \times 10^{2}\right.$ to $\left.9.3 \times 10^{3} \mathrm{~g} \mathrm{C} \mathrm{m}^{-2} \mathrm{y}^{-1}\right)$. Although exported biomass is likely entrained into buoyant plumes that disperse widely (Lavelle and Wetzler, 1999), this productivity would still equal background (areal) photosynthetic flux to the deep sea if diluted into an area of $\approx 1-25 \mathrm{~km}^{2}$. Therefore, while subseafloor chemosynthesis can vastly increase carbon for consumers locally, it will be ultimately confined to a relatively small area near active venting. A recent study showed heterotrophic bacteria with the potential to degrade diverse organic substrates are clustered around low-temperature vents (Meier et al., 2016), which

\footnotetext{
${ }^{1}$ See methods for details
} 
may indicate that chemosynthetic production is closely coupled to remineralization.

Compared to primary productivity, subseafloor biomass standing stock is even more poorly constrained. We estimated a minimum value for biomass standing stock by first assuming that cell-specific rates of oxygen/nitrate reduction (McNichol et al., 2016) are maximum rates for natural populations. We then determined the minimum number of cells needed to account for depletions of chemosynthetic substrates observed. Finally, using a conservative value of $\approx 173 \mathrm{fg} \mathrm{C}$ cell $^{-1} \quad 2$, we calculated a minimum standing stock of the subseafloor biosphere at Crab Spa of $\approx 17 \mathrm{~g} \mathrm{C}^{2}$.

If we assume our values for primary productivity approximate export production, the average residence time of subseafloor biomass under steady-state conditions would be very short - approximately $10 \mathrm{~h}$. Microbial doubling times would need to be the same on average to maintain subseafloor biomass; indeed, $10 \mathrm{~h}$ is consistent with isolates of chemoautotrophic Epsilonproteobacteria (Nakagawa and Takai, 2008). Although direct measurements of standing stock in the subseafloor biosphere may not be possible, in situ growth rates could be estimated by determining the frequency of dividing cells (Hagström et al., 1979). If this was similar to the residence time derived here, it would suggest our estimates are accurate.

Taking an estimated global low-temperature hydrothermal flux of $2.8-5.6 \times 10^{15} \mathrm{~kg} \mathrm{y}^{-1}$ (Elderfield and Schultz, 1996), we calculated chemosynthetic subseafloor primary production at deep-sea vents to be $2.9 \times 10^{11}-1.4 \times 10^{12} \mathrm{~g} \mathrm{C} \mathrm{y}^{-1}$ - at most $\approx 0.5 \%$ of photosynthetic primary productivity reaching these depths (Lampitt and Antia, 1997). Our global estimate is comparable to McCollom and Shock (1997), despite the aforementioned discrepancy in carbon fixed per L end-member fluid. This is because extrapolations in McCollom and Shock (1997) were based on global high-temperature hydrothermal fluid flux instead of the higher values reported for low-temperature fluid flux used in our study (Elderfield and Schultz, 1996).

We also calculated a minimum global standing stock of $1.42 .7 \times 10^{9} \mathrm{~g} \mathrm{C}$, three orders of magnitude lower than $7.4 \times 10^{12} \mathrm{~g} \mathrm{C}$ estimated from a thermodynamic framework (Nakamura and Takai, 2015). Our standing stock represents a minimum value; if percell rates in situ were slower, or if cell-specific rates were similar but carbon per cell was higher, our estimate would increase. In Nakamura and Takai (2015), the authors assumed a maintenance energy for non-growing cells to calculate the standing stock biomass, which

\footnotetext{
${ }^{2}$ See methods for details
} 
is not realistic based on our results showing microbes are active and grow rapidly.

Naturally, these extrapolations have several caveats. Firstly, CGE may be different for other putative autotrophs such as the SUP05-cluster found at vents (Anderson et al., 2013). Although CGE has not been measured for SUP05, related autotrophic Gammaproteobacteria do not differ considerably from our values (Klatt and Polerecky, 2015), indicating this will likely be a small bias. Similarly, while differing fluid chemistry may also affect microbial productivity, we only observed a 2-fold difference in CGE between diverse amendments (Fig 3-3). By contrast, much larger uncertainties exist in estimating the flux and residence time of fluids in the subseafloor biosphere (Elderfield and Schultz, 1996), rendering estimates of global chemosynthetic primary productivity highly uncertain.

In our study, empirical measurements of growth efficiency allowed us to directly constrain in situ biogeochemical processes. Our study also revealed insights into the physiological diversity of active communities of autotrophic Epsilonproteobacteria, which are dominant players at low-temperature deep-sea hydrothermal vents world-wide and other habitats characterized by sulfide-oxygen interfaces (Huber et al., 2007; Nakagawa and Takai, 2008; Campbell et al., 2006). Our approach is broadly applicable to incubations of deep-sea microbial communities, and could be used to quantify the effect of diverse, uncultivated microbes on global biogeochemical cycles.

\subsection{Acknowledgements:}

We thank the officers, crew, and pilots of the R/V Atlantis and ROV Jason for their expert help at sea and their outstanding efforts acquiring the samples needed for this study. We also thank the scientific party for support, with special thanks going to Kerry McCulloch, Miriam Sollich, and Xi Wei for invaluable help with shipboard lab experiments. Many thanks are also due to Ben van Mooy for lending his lab's oxygen optode system, to Scott Wankel, Carly Buchwald and Zoe Sandwith for help quantifying nitrate/nitrite after the cruise, to Virginia Edgcomb for lending her lab's Aanderaa in situ oxygen optode, and to Jeremy Rich for providing nitrate measurements for background seawater. Thanks are also due to Marc MuSSmann and Stefan Dyksma for help designing and testing CARD-FISH probes, and to the Kujawinski lab for providing measurements of DOC in Crab Spa fluids. Finally, thanks also to Lubos Polerecky for kindly answering questions about how to analyze NanoSIMS 
data.

This research was funded by a grant of the Dimensions of Biodiversity program of the National Science Foundation to S.M.S and J.S.S. (NSF-OCE-1136727). Funding for J.M. was provided by doctoral fellowships from the National Sciences and Engineering Research Council of Canada (NSERC-PGSD3) and the National Aeronautics and Space Administration Earth Systems Science Fellowship (NESSF), an award from the Canadian Meterological and Oceanographic Society, as well as funding from WHOI academic programs and the aforementioned National Science Foundation grant. The author is grateful for use of the analytical facilities of the Centre for Chemical Microscopy (ProVIS) at the Helmholtz Centre for Environmental Research, Leipzig which is supported by European Regional Development Funds (EFRE - Europe funds Saxony) and the Helmholtz Association. 


\section{Chapter 4}

\section{A Physiologically-Reconciled Model of an Epsilonproteobacterial \\ Chemolithoautotroph}

This chapter is a paper currently in preparation with co-authors Jon Steffensen, Ying Zhang and Stefan M. Sievert. 


\subsection{Abstract:}

Chemoautotrophic Epsilonproteobacteria are often dominant in sulfidic, oxygen-depleted environments where they use reduced inorganic chemicals to fuel their growth. Despite their ecological importance, the biochemical mechanisms that support their growth remain poorly understood. Here, we report a theoretical model of biochemical pathways used by Sulfurimonas denitrificans, a sulfur- and hydrogen-oxidizing microbe that can use oxygen and nitrate as terminal electron acceptors. Since several essential metabolic pathways needed to support its growth were missing or incompletely described, we used data from other organisms to posit hypothetical biochemical mechanisms involving complexes found in this organism's genome. Then, by comparing in silico growth yields with chemostat yield data, we inferred the energy conservation efficiency of these complexes such that the model accurately reproduced experimental growth yields. Three key mechanisms are central to our model. Firstly, sulfur oxidation by the SOx enzyme complex donates electrons to the membrane quinone pool, possibly via a sulfide:quinone oxidoreductase-like protein. Secondly, the high-affinity cytochrome oxidase in this organism is predicted to be non-electrogenic, and obtain its chemical protons for the reduction of $\mathrm{O}_{2}$ to $\mathrm{H}_{2} \mathrm{O}$ from the periplasm. Thirdly, reduced ferredoxin for the reverse tricarboxylic acid cycle is produced via reverse electron transport via a non-canonical complex I. If proved correct by experimental tests outlined herein, these mechanisms would help understand the fundamental physiology of autotrophic Epsilonproteobacteria and their role in the natural environment.

\subsection{Introduction:}

At deep-sea hydrothermal vents, chemolithoautotrophic microbes support entire food webs by fixing inorganic carbon into biomass (Jannasch and Mottl, 1985). Bacteria from the subdivision Epsilonproteobacteria often dominate deep-sea vent communities, and are found in many other sulfidic environments (Campbell et al., 2006). Pure culture studies have shown that autotrophic Epsilonproteobacteria fix carbon by coupling the oxidation of hydrogen and reduced sulfur compounds to the reduction of nitrate, oxygen and elemental sulfur (Nakagawa and Takai, 2008; Sievert and Vetriani, 2012). Because of their key role in chemosynthetic ecosystems, a quantitative understanding of their metabolism would help model ecological and biogeochemical processes. 
Genome-scale metabolic modeling (GSMM) is a process whereby computationally predicted enzymatic reactions are combined together to create an in silico model of cellular metabolism (Durot et al., 2009). Construction of a metabolic network requires manual curation, and will inevitably reveal gaps in biochemical knowledge for the organism in question. This process may itself be useful as it can lead to the discovery of novel enzymes or pathways. Once assembled, another important use of the metabolic network is to predict biological growth yields under different conditions. This is accomplished by first defining a biomass function, which is a simplified representation of fundamental components necessary to build a cell. For example, a biomass function includes amino acids, nucleotides, lipids and vitamins but not more complex biological polymers such as proteins or DNA/RNA. Next, the model is grown in silico with the desired medium composition and the biomass function defined as the model objective. This is accomplished by using flux balance analysis (FBA) to search for a solution optimizing biomass yield by treating the network of metabolic reactions as a series of linear equations (Orth et al., 2010). Finally, by comparing growth yields between different medium compositions, data is produced that can be directly compared to physiological studies.

This approach is currently difficult to apply to autotrophic Epsilonproteobacteria however, because the biochemical mechanisms of chemosynthetic enzymes are largely unknown. For example, how reducing power needed for carbon fixation (i.e. NAD(P)H/reduced ferredoxin) is produced from a high redox potential electron donor like thiosulfate remains completely unknown (Marshall et al., 2012). Therefore at present, the application of GSMMFBA to these organisms requires inferences on the putative biochemical mechanisms supporting their growth. Since GSMM-FBA simulates growth yields in silico, it can be compared with in vivo data from pure cultures. If yields are in agreement, it implies predicted biochemical mechanisms are reasonable. If in disagreement, it points to gaps in the current knowledge of these organisms.

For this study, we applied this theoretical modeling approach to the epsilonproteobacterium Sulfurimonas denitrificans (Timmer-Ten Hoor, 1975; Takai et al., 2006). In addition to having a well-annotated genome (Sievert et al., 2008), S. denitrificans is currently the best-characterized species amongst autotrophic Epsilonproteobacteria (Timmer-Ten Hoor, 1975, 1981; Han and Perner, 2014, 2016). Below, we discuss how we derived a best-guess for autotrophic metabolism in S. denitrificans that is consistent with chemostat yield data 
and the biochemistry of related organisms. We further suggest experiments to test these hypotheses about the core metabolism of S. denitrificans. If verified, these mechanisms will have important implications for understanding the bioenergetics and evolution of diverse autotrophic Epsilonprotebacteria.

\subsection{Materials and Methods:}

\subsubsection{Genome-scale metabolic modeling:}

The function of genes in Sulfurimonas denitrificans (Sievert et al., 2008) was first predicted using a consensus annotation approach (Zhang and Sievert, 2014) and subsequently mapped to the modelSEED database (Devoid et al., 2013) to produce equations representing metabolic reactions. These reactions formed the basis of an initial draft model. An objective biomass function was then defined based on the Helicobacter pylori iIT341 model (Thiele et al., 2005). Several additions were made based on pathways predicted by the modelSEED database. Siroheme was added with the same coefficient as haem, as well as pyridoxine phosphate (vitamin B6). For the synthesis of each type of membrane lipid, the modelSEED database predicted several separate pathways producing different lipid chain lengths; in contrast, the iIT341 model biomass function uses only one chain length per lipid type. In our model, separate reactions predicted by modelSEED were retained, and the coefficient provided in iIT341 biomass function for pooled lipid classes was divided by the number of different chain lengths predicted by modelSEED.

At this point, flux balance analysis (FBA) was attempted to determine the objective flux for the biomass function. However, biomass could not be produced, so a custom script was developed to identify reactions that contained dead-end metabolites; these reactions were then manually curated. Following this procedure, biomass flux remained at zero. Therefore, the FBA model objective was set to each biomass constituent to determine which could be produced, and broken biomass constituents were resolved by manual curation. For both the biomass function and dead-end metabolites, curation was accomplished by removing unneeded reactions, adding custom reactions (denoted with the prefix "jm"), or by using already-existing modelSEED reactions. In some cases, cofactors and/or directionality were changed from the modelSEED annotations to allow flux through reactions. Both have been noted in reaction prefixes by changing the case of either the ' $r$ ' or ' $x$ ' for reversibility and 
changes, respectively. For example, RXn00001 would differ from the default modelSEED reaction (rxn00001) in that it is reversed ('R') and one or more cofactor was changed ('X'). At this stage of curation, the model was able to produce biomass with at least some energy sources during FBA.

Table 4.1: Model summary statistics.

\begin{tabular}{cc}
\hline Parameter & Number \\
\hline Original predicted reactions (modelSEED) & 768 \\
Remaining predicted reactions & 503 \\
Custom reactions & 12 \\
Custom transfer reactions & 19 \\
Reactions added from modelSEED database & 32 \\
Custom source and sink reactions & 8 \\
Transfer reactions from modelSEED database & 9 \\
Total number of non-biomass reactions & 583 \\
\hline
\end{tabular}

The model was further curated by constraining the directionality and reversibility of metabolic reactions. Initially, the annotations from the modelSEED database were applied to the model. However, this rendered the model unable to produce biomass. Therefore, directionality of reactions was manually curated, revealing that several irreversible reactions in the modelSEED (rxn00053, rxn00550, rxn00518-00524, rxn00559-00565 and rxn00629) needed to be reversible in order for the model to produce biomass. Reactions were then manually inspected to constrain those for which directionality was not specified in the modelSEED database but are known to be irreversible (e.g. denitrification enzymes). Similar logic was applied to reactions that involving soluble electron carriers such as NAD $(\mathrm{P}) \mathrm{H}$ and ferredoxin or thermodynamically-irreversible reactions involving ATP or other nucleoside phosphates. Additionally, FBA output was inspected for unrealistically high fluxes which indicate thermodynamically-impossible loops producing electron donors or ATP for biosynthesis. Summary statistics describing this curation process are presented in Table 4.1. 
After biomass flux was non-zero and directionality was constrained, more specific curation of core metabolic reactions was carried out in order to reproduce the metabolic potential of this organism (Timmer-Ten Hoor, 1981; Sievert et al., 2008; Han and Perner, 2014). These reactions were based on Fig. 2 from Sievert et al. (2008) with the following modifications. The cytochrome $\mathrm{cbb}_{3}$ uses only oxygen (not nitric oxide), NorBC is the only nitric oxide reductase and NosZ accepts electrons from cytochrome c only. Both thiosulfate and sulfide oxidation are modeled as complete oxidation to sulfate, transferring electrons to menaquinone instead of cytochrome c as previously described for the SOx complex (Dahl et al., 2008). Neither polysulfide reductase or formate oxidation were included, and reverse electron transport was modeled as discussed below.

Since it is uncertain which electron donor other than ferredoxin (i.e. NADH, NADPH or both) are used for biosynthetic reactions in the cell, a reversible interconversion reaction between NADPH and NADH was introduced (rxn00083) to reflect this uncertainty.

Energy-generating metabolic reactions of $S$. denitrificans likely take place in the periplasm (Sievert et al., 2008); compounds in the model were therefore localized into external (e), periplasmic (p) and cytoplasmic compartments. In order to distinguish between protons produced during redox reactions and those contributing to the proton-motive force (pmf), periplasmic protons were used as a unique tracer of proton translocation. For protons produced/consumed in periplasmic reactions not contributing to the pmf, protons in the external compartment were the source/sink for these reactions. Whether periplasmic or cytoplasmic protons were used for menaquinone reduction or vectorial proton transport was determined according to Simon et al. (2008) or via logic detailed below.

Several functions posited are not supported by direct biochemical evidence. Even for those complexes whose function has been identified in other organisms (e.g. cytochrome $\mathrm{cbb}_{3}$ oxidase), the stoichiometry of of proton translocation in vivo is unknown. For these complexes, several hypothetical mechanisms and/or proton pumping stoichiometry were considered. To identify which was most likely to be correct, in silico growth efficiency was compared with chemostat growth yields of Sulfurimonas denitrificans. If predicted growth efficiency was close to experimental data, the mechanism was considered plausible and viceversa.

Once the model reproduced experimental observations, it was subjected to consistency checks using the PSAMM software (Steffensen et al., 2016) which verified reaction stoichiom- 
etry and identified reactions that have zero flux under all conditions. In order for PSAMM to run the model, the metabolite "no" was changed to "nox" since "no" is interpreted as a boolean "false" by PSAMM. The exact solver QSoptEX was used due to limitations in the CPLEX solver for identifying reactions with very low fluxes (e.g. heme biosynthesis). Of the reactions that can carry no flux (according to the fluxcheck tool), only two were kept in the model, both related to nitrate transport into the cytoplasm, which may provide a source for posited assimilatory nitrate reduction (Sievert et al., 2008). For additional annotation of the Sulfurimonas denitrificans genome, operons were predicted using OperonPredicter (Taboada et al., 2010) and transmembrane domains were predicted using the TMHMM program (Sonnhammer et al., 1998).

\subsubsection{Chemostat cultivation and growth yield measurements:}

A custom-built chemostat was used to grow Sulfurimonas denitrificans. Triplicate temperaturecontrolled vessels with a liquid volume of $200 \mathrm{~mL}$ were fed via a peristaltic pump at an average dilution rate of $0.57 \mathrm{~d}^{-1}$. Medium was kept anoxic by constant flushing with an anoxic gas mixture at a slight overpressure $(27.5 \mathrm{KPa})$. This overpressure also served to remove excess medium via a siphon located in chemostat vessels. Vessels were stirred constantly with magnetic stir bars, and all wettable surfaces were either borosilicate glass or PTFE.

The standard medium recipe was made by first dissolving and autoclaving the following components in MilliQ water (per L final volume): $\mathrm{NaCl} 10 \mathrm{~g} ; \mathrm{NH}_{4} \mathrm{Cl} 1 \mathrm{~g}, \mathrm{MgSO}_{4} \bullet 7 \mathrm{H}_{2} \mathrm{O} 3.5$ g; $\mathrm{CaCl}_{2} \bullet 2 \mathrm{H}_{2} \mathrm{O} 0.42 \mathrm{~g} ; \mathrm{KCl} 0.7 \mathrm{~g} ; \mathrm{KNO}_{3} 2.0 \mathrm{~g}$; Trace element solution SL-6 (DSMZ 465) $1 \mathrm{~mL}$. After autoclaving, separately-sterilized anaerobic solutions of the following components were added (per L final volume): $\mathrm{NaHCO}_{3} 5 \mathrm{~g} ; \mathrm{KH}_{2} \mathrm{PO}_{4} 0.5 \mathrm{~g}$; selenate/tungstate solution (DSMZ 944a) $4 \mathrm{~mL} ; 0.7 \mathrm{~mL}$ of $2 \mathrm{mg} \bullet \mathrm{mL}^{-1} \mathrm{FeCl}_{3}$ in $0.1 \mathrm{~N} \mathrm{H}_{2} \mathrm{SO}_{4} ; \mathrm{Na}_{2} \mathrm{~S}_{2} \mathrm{O}_{3} \bullet 5 \mathrm{H}_{2} \mathrm{O} 5 \mathrm{~g}$. The thiosulfate solution was sterilized by filtering through a $0.2 \mu \mathrm{M}$ membrane and the bicarbonate solution was autoclaved under a $\mathrm{N}_{2} / \mathrm{CO}_{2}$ (80:20) atmosphere.

For cultivation with $\mathrm{H}_{2} / \mathrm{NO}_{3}{ }^{-}$as energy sources, thiosulfate was omitted and $\mathrm{H}_{2} / \mathrm{CO}_{2}$ (80:20) was provided as the headspace gas. For cultivation with $\mathrm{S}_{2} \mathrm{O}_{3}{ }^{2-} / \mathrm{NO}_{3}{ }^{-}$, the headspace gas was $\mathrm{N}_{2} / \mathrm{CO}_{2}$ (80:20). For cultivation with $\mathrm{S}_{2} \mathrm{O}_{3}{ }^{2-} / \mathrm{O}_{2}$, a microaerobic environment was created by mixing $\mathrm{N}_{2} / \mathrm{CO}_{2}$ (80:20) with pure $\mathrm{O}_{2}$ to a final concentration of $\approx 1 \%$ air saturation using a mass-flow controller (Tylan 260 series with RO-28 control box). Oxygen concentrations in chemostat vessels were monitored in real time with Pts3 optodes (Presens, 
Germany).

Cells were enumerated after fixation with borate-buffered formalin, DAPI staining and filtration onto black $0.2 \mu \mathrm{M}$ polycarbonate filters (Sherr et al., 2001). Once numbers stabilized after 6-9 days of cultivation, both source medium and chemostat vessels were sampled for cell carbon (CC) and ash-free dry weight (AFDW). Combusted and pre-weighed GF-75 filters (0.3 $\mathrm{\mu M}$ pore size; Advantec) were used for both measurements. For AFDW, filters were first wetted with $0.5 \mathrm{M}$ ammonium formate (Zhu and Lee, 1997). Next, $20 \mathrm{~mL}$ of medium or cells were filtered, followed by $10 \mathrm{~mL}$ of ammonium formate. Cells were dried overnight at $80^{\circ} \mathrm{C}$, placed in a vacuum desiccator, and weighed when cool. Then, filters were combusted at $480^{\circ} \mathrm{C}$ for $8 \mathrm{~h}$ and weighed as before. Medium blanks were subtracted to determine AFDW. For cell carbon, $20 \mathrm{~mL}$ of both medium and cell culture were filtered using pre-combusted glassware. Filters were stored in pre-combusted shell vials in $-80^{\circ} \mathrm{C}$ prior to analysis. Total cell carbon was determined by acidification and subsequent combustion of biomass.

For comparisons between hydrogen/nitrate and thiosulfate/nitrate, growth yield data were normalized to total nitrate consumed. For comparisons between thiosulfate/nitrate and thiosulfate/oxygen yields were normalized to total sulfate produced. Ratios of growth yields were used to compare to model results.

\subsection{Results:}

\subsubsection{Chemostat cultivation:}

For all incubation conditions, $\mathrm{pH}$ measured daily was $7.19 \pm 0.04$ for $\mathrm{H}_{2} / \mathrm{NO}_{3}{ }^{-}, 7.06 \pm$ 0.01 for $\mathrm{S}_{2} \mathrm{O}_{3}{ }^{2-} / \mathrm{NO}_{3}{ }^{-}$, and $7.02 \pm 0.02$ for $\mathrm{S}_{2} \mathrm{O}_{3}{ }^{2-} / \mathrm{O}_{2}$. Upon sampling, cell numbers had not varied on average by more than $11 \%$ over at least 2 days of cultivation, indicating steady state had been reached. This occurred after 7,8 , and 5 days for $\mathrm{H}_{2} / \mathrm{NO}_{3}{ }^{-}, \mathrm{S}_{2} \mathrm{O}_{3}{ }^{2-} / \mathrm{NO}_{3}{ }^{-}$, and $\mathrm{S}_{2} \mathrm{O}_{3}{ }^{2-} / \mathrm{O}_{2}$ respectively.

\subsubsection{Curation of ModelSEED-predicted reactions:}

While the ModelSEED database is a powerful tool for producing an initial draft model, it must be manually curated. Below, we describe decisions made during this process and justification for these choices. 
Firstly, we observed that there were several reactions that suggested potential heterotrophic metabolism. One gene (Suden_0620) was predicted to be involved in metabolism of dipeptides, potentially abundant in the sediment environment from which S. denitrificans was isolated. However, S. denitrificans has few predicted peptidases $(\approx 40)$ compared to the heterotroph E. coli K12 ( $\approx 600)$ (Rawlings et al., 2010), which could suggest the peptidases present in S. denitrificans are involved in other processes such as cell division.

Similarly, ModelSEED predicted a galactose or glucose transporter from one gene (Suden_2077). However, the Pfam database (Finn et al., 2016) shows that this protein is part of the major facilitator family, which has a wide range of substrates not restricted to sugars (Pao et al., 1998). Given that the goal of this model was to reproduce autotrophic metabolism, and that there are considerable uncertainties surrounding these putative heterotrophic pathways, they were removed from the model.

The curation process also revealed pathways not previously noted for S. denitrificans. Three terminal reactions involved in the biosynthetic pathway of B12 are present, but not the preceding 14 biosynthetic steps. This observation suggests that B12 precursors may be taken up from the environment by $S$. denitrificans, possibly using putative cobalamin transporters identified by ModelSEED (Suden_0115, 1538). Supporting this potential role, both transporter genes are found within operons with TonB-dependent receptors, and the former is adjacent to genes that encode putative cobalamin-modifying functions (e.g. Suden_0112). In addition, the genome contains two different methionine synthases, a B12-independent (Suden_0899) and -dependent version (Suden_2031), which would allow growth without this vitamin, as observed in pure culture (Timmer-Ten Hoor, 1975). However, given the uncertainties associated with this pathway, it was also removed from the model.

Nearly full pathways were present for spermidine and putrescine synthesis from arginine. In order to recycle 5'-S-methyl-5'-thioadenosine produced in spermidine synthesis, rxn01021 was added and rxn00127 was added to produce s-adenosylmethioninamine from the decarboxylation of SAM, required to synthesize spermidine. A nearly complete pathway for biotin synthesis is also present in $S$. denitrificans, but pimelate, a $\mathrm{C}_{7}$ dicarboxylic acid required for biotin synthesis was not produced in the model. Since the biosynthesis of pimelate remains obscure (Lin and Cronan, 2011), it was provided to the model to support biotin synthesis.

In addition to heme, S. denitrificans also contains a gene for siroheme biosynthesis 
(Suden_1977), which is needed for both the synthesis of cytochrome cd $_{1}$-containing nitrite reductase (Zumft, 1997) and assimilatory sulfate reductases (Murphy and Siegel, 1973) and was therefore added into the biomass function with the same coefficient as heme. Siroheme, an essential cofactor in nitrate and sulfur reducing enzymes, is synthesized anaerobically (Spencer et al., 1993), but heme synthesis can occur with either oxygen-requiring or -independent enzymes. Both of these were predicted by modelSEED (Suden_1654, Suden_0606 respectively) and included in the model.

For assimilation of sulfur into biomass, modelSEED predicted S. denitrificans can either incorporate thiosulfate or sulfide into proteins or carry out assimilatory sulfate reduction. However, biotin synthase also requires a reduced sulfur compound, which in vivo is likely provided by an iron sulfur cluster (Lin and Cronan, 2011). In the modelSEED reaction scheme, this is represented as cytoplasmic elemental sulfur, which was not produced in the model. To connect biotin synthesis to pathways of sulfur assimilation, we provided two custom reactions for biotin synthesis that use reduced sulfur compounds present in the model (rXn08180_h2s and rXn08180_h2s2o3). For some thiosulfate assimilation reactions, sulfite or sulfate is produced in the cytoplasm according to the modelSEED or our custom reactions (e.g. rxn05733, rXn08180_h2s2o3). Since these by-products prevented flux through these sulfur assimilation reactions, they were removed by providing sink reactions for sulfate and sulfite.

\subsubsection{Core chemoautotrophic enzymes:}

Once the metabolic network produced biomass, it still did not replicate certain aspects of in vivo activity. For example, in silico growth with thiosulfate and nitrate was not possible - the original condition this organism was isolated under (Timmer-Ten Hoor, 1975). This was because no electron transport pathway linked thiosulfate with the membrane quinone pool - necessary for the initial step of nitrate reduction. In contrast, growth with hydrogen and nitrate was possible because the hydrogenase present donated electrons to the quinone pool.

In the genome description of $S$. denitrificans, the SOx system was suggested to be responsible for thiosulfate oxidation (Sievert et al., 2008). However, the SOx system is currently thought to be a soluble periplasmic membrane complex (Friedrich et al., 2001), and therefore cannot donate electrons to the quinone pool. Although a thiosulfate:quinone 
oxidoreductase (TQR) responsible for partial oxidation of thiosulfate to tetrathionate has been described (Müller et al., 2004), neither this complex nor any other known thiosulfateoxidizing enzymes are present in the genome of S. denitrificans. Therefore, it seems likely that a yet undescribed complex serves to link the SOx system to the membrane quinone pool in S. denitrificans. Considering this, we employed a mechanism whereby the complete eight-electron oxidation of thiosulfate and sulfide to sulfate was linked to the reduction of menaquinone via the SOx system (Fig 4-1a).

To support carbon fixation, $\mathrm{NAD}(\mathrm{P}) \mathrm{H}$ and ferredoxin are both required for the reverse tricarboxylic acid (rTCA) cycle; however, no mechanism for ferredoxin production is known for S. denitrificans (Marshall et al., 2012). However, it has been reported that the heterotrophic epsilonproteobacterium Campylobacter jejuni possesses a non-canonical NADH:ubiquinone oxidoreductase complex (NUO) that oxidizes reduced flavodoxin instead of NADH (Weerakoon and Olson, 2008). Such a complex has the potential to reduce ferredoxin if run in reverse; indeed, S. denitrificans has a non-canonical NUO operon similar to C. jejuni. However, $\mathrm{NAD}(\mathrm{P}) \mathrm{H}$ is also needed, and not produced by such a mechanism. Therefore, for modeling purposes, we included a hypothetical mechanism that reduces both $\mathrm{NAD}(\mathrm{P})+$ and ferredoxin simultaneously via an electron-bifurcation-like mechanism (Fig $4-2)$. 

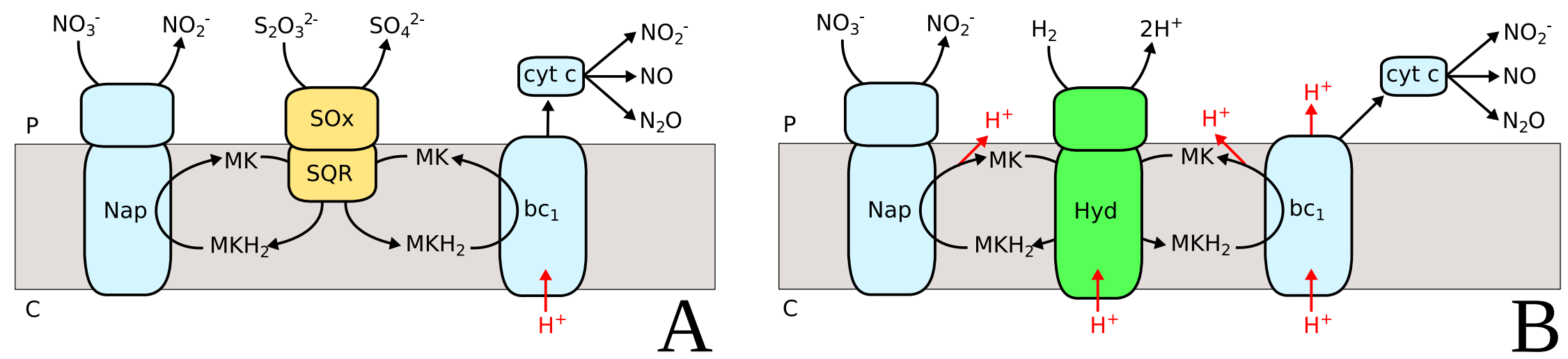

Figure 4-1: Hypothetical mechanisms of electron transport and energy conservation during denitrification in Sulfurimonas denitrificans with thiosulfate (A) or hydrogen (B) as an electron donor. Black arrows indicate electron transport, and red arrows contributions to the proton-motive force. Nap=Nap nitrate reductase complex; $\mathrm{SOx}=\mathrm{SOx}$ sulfur oxidation complex; $\mathrm{SQR}=$ Sulfide:quinone oxidoreductase; $\mathrm{bc}_{1}=$ Complex III; Hyd=Hydrogenase; $\mathrm{MK} / \mathrm{MKH}_{2}=$ Oxidized/reduced menquinone; cyt $\mathrm{c}=$ Soluble cytochrome. $\mathrm{P}=\mathrm{Periplasm}$; $\mathrm{C}=\mathrm{Cyto}-$ plasm. 
Because of this hypothetical reverse electron transport mechanism, a sink was now required for reduced ferredoxin. While $\mathrm{NAD}(\mathrm{P}) \mathrm{H}$ and reduced ferredoxin can be consumed in a 1:1 ratio by the $\mathrm{rTCA}$ cycle, more $\mathrm{NAD}(\mathrm{P}) \mathrm{H}$ is required for biosynthetic reactions (e.g. de novo lipid synthesis) than reduced ferredoxin, which only participates in a few pathways. Therefore, a sink for reduced ferredoxin was required in order for core biosynthetic reactions to proceed. When nitrate was provided, assimilatory reduction of nitrite to ammonium via ferredoxin:nitrite oxidoreductase provided a sink. However, under aerobic conditions the model did not produce biomass; therefore, another sink was needed. A review of the literature revealed that $S$. denitrificans contains a gene highly similar to the previously characterized ferredoxin-dependent glutamate synthase of Hydrogenobacter thermophilus (Kameya et al., 2007) (Suden_1176). The addition of this reaction to the model permitted aerobic growth, and removed flux through the ferredoxin:nitrite reductase under denitrifying conditions.

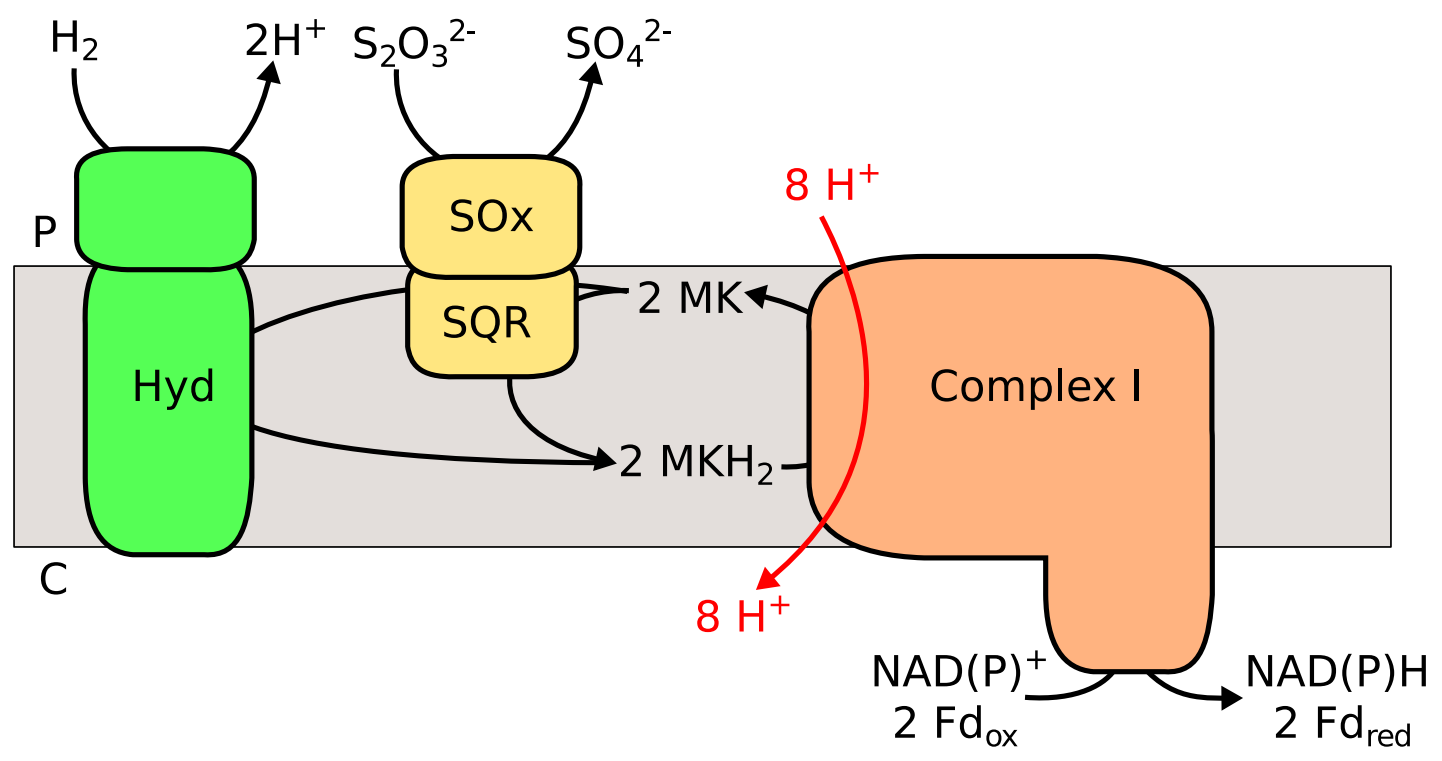

Figure 4-2: Hypothetical mechanism of reverse electron transport in Sulfurimonas denitrificans. Black arrows indicate electron transport, and red arrows protons used to drive endergonic reverse electron transport. Either hydrogen or thiosulfate can donate electrons to complex I in this scheme. Hyd $=$ Hydrogenase; $\mathrm{SOx}=\mathrm{SOx}$ sulfur oxidation complex; $\mathrm{SQR}=$ Sulfide:quinone oxidoreductase $\mathrm{MK} / \mathrm{MKH}_{2}=$ Oxidized/reduced menquinone; $\mathrm{Fd}=$ soluble ferredoxin; $\mathrm{P}=$ Periplasm; $\mathrm{C}=$ Cytoplasm.

This imbalance would also be eliminated if there was a mechanism to interconvert 
ferredoxin and $\operatorname{NAD}(\mathrm{P}) \mathrm{H}$. Maurice et al. (2007) report the activity of a flavodoxin:quinone reductase (FqrB) that reversibly interconverts NADPH and flavodoxin in C. jejuni. A homolog in S. denitrificans (Suden_0165) was detected in a proteome at similar abundances to rTCA enzymes (Götz et al., unpublished), suggesting it fulfills an important function in vivo; therefore, we included this reaction. Since under autotrophic conditions both ferredoxin and $\mathrm{NAD}(\mathrm{P}) \mathrm{H}$ pools are likely predominantly reduced, the FqrB reaction is not likely to be reversible as in C. jejuni (Maurice et al., 2007) and this was reflected in the reaction.

\subsubsection{Energy Conservation Efficiency of Enzymatic Complexes:}

Once the model produced biomass under conditions described previously (Timmer-Ten Hoor, 1981; Sievert et al., 2008; Han and Perner, 2014), FBA was used to compare in silico and in vivo growth yields. At first, several discrepancies were noted. Firstly, growth efficiencies

did not differ between thiosulfate and hydrogen oxidation, in contrast to data previously reported in batch culture (Han and Perner, 2014) and chemostat growth data presented here (Table 4.2). Secondly, the in silico ratio between aerobic and denitrifying growth efficiency was much higher than what has previously been determined in chemostat experiments (Timmer-Ten Hoor, 1981). 
Table 4.2: Growth yields predicted from in silico metabolic modeling compared with growth yields during chemostat cultivation

\begin{tabular}{|c|c|c|c|c|c|c|}
\hline \multirow[b]{2}{*}{ Growth Condition } & \multirow{2}{*}{$\begin{array}{l}\text { In silico growth yield } \\
\text { (Eq to } \mathrm{CO}_{2} \text { fixation / Eq oxidized) }\end{array}$} & \multicolumn{2}{|c|}{$\mathrm{Y}^{*}{ }_{S O 4}$ (this study) } & \multicolumn{3}{|c|}{$\mathrm{Y}^{*}{ }_{S 2 O 3}$ (Timmer Ten-Hoor, 1981) } \\
\hline & & AFDW & $\mathrm{CC}$ & DW & $\mathrm{CC}$ & $\mathrm{CP}$ \\
\hline $\mathrm{S}_{2} \mathrm{O}_{3}{ }^{2-} / \mathrm{NO}_{3}^{-}$ & 0.67 & $0.89 \pm 0.14$ & $0.67 \pm 0.14$ & 5.15 & 2.36 & 1.77 \\
\hline $\mathrm{S}_{2} \mathrm{O}_{3}{ }^{2-} / \mathrm{O}_{2}$ & 1.03 & $1.49 \pm 0.20$ & $0.91 \pm 0.12$ & 7.74 & 4.00 & 3.90 \\
\hline Ratio & 1.54 & 1.67 & 1.36 & 1.50 & 1.70 & 2.20 \\
\hline
\end{tabular}

\begin{tabular}{|c|c|c|c|c|}
\hline Growth Condition & (Eq to $\mathrm{CO}_{2}$ fixation / Eq oxidized) & AFDW & $\mathrm{CC}$ & AFDW $=$ Ash-free dry weight $\left(\mathrm{g} \mathrm{M}^{-1}\right)$ \\
\hline $\mathrm{S}_{2} \mathrm{O}_{3}{ }^{2-} / \mathrm{NO}_{3}{ }^{-}$ & 0.95 & $1.01 \pm 0.22$ & $0.76 \pm 0.21$ & $\mathrm{CC}=$ Cell carbon $\left(\mathrm{g} \mathrm{C} \mathrm{M}^{-1}\right)$ \\
\hline \multirow[t]{2}{*}{$\mathrm{H}_{2} / \mathrm{NO}_{3}^{-}$} & 2.58 & $2.45 \pm 0.73$ & $1.75 \pm 0.31$ & DW $=$ Dry weight $\left(\mathrm{g} \mathrm{M}^{-1}\right)$ \\
\hline & & & & $\mathrm{CP}=$ Cell protein $\left(\mathrm{g}\right.$ protein $\left.\mathrm{M}^{-1}\right)$ \\
\hline Ratio & 2.72 & 2.43 & 2.30 & Units for in silico modeling arbitrary \\
\hline
\end{tabular}

Errors for AFDW and CC are standard deviations from biological triplicates 
The first discrepancy - why modeled growth yields in silico were identical during sulfur and hydrogen oxidation but different in growth experiments - was resolved by considering the inferred structure of enzyme complexes. The hydrogenase in S. denitrificans likely has a transmembrane domain - allowing it to accept protons from the periplasm during quinone reduction (Fig 4-1b) (Gross et al., 1998; Sievert et al., 2008). When coupled to a quinolaccepting reductase, this translocates protons via a redox-loop mechanism (Simon et al., 2008). In contrast, proteins catalyzing sulfur oxidation coupled to quinone reduction have not been demonstrated to translocate protons. This is because they either do not have transmembrane domains (Griesbeck et al., 2002) or the quinone-accepting site faces the cytoplasm (Müller et al., 2004). Since thiosulfate oxidation likely occurs in the periplasm in $S$. denitrificans, we chose to model the SOx system with a periplasmic-facing non-transmembrane anchor similar to sulfide:quinone oxidoreductase (SQR; Fig 4-1a). With this mechanism in place, in silico denitrifying growth efficiency was congruent with experimental data (Table $4.2)$.

The second inconsistency why large differences in growth yields between aerobic and denitrifying respiration were observed in silico but only a small difference observed during experiments - could be reconciled if the energy conservation efficiency of aerobic respiration was either lower or that of denitrification was higher. However, proton translocation is not thought to be catalyzed by denitrification enzymes (Chen and Strous, 2013). Additionally, if denitrification enzymes were capable of energy conservation, the overall growth yield of $S$. denitrificans would be much higher in silico than in vivo (Timmer-Ten Hoor, 1981; Klatt and Polerecky, 2015). For these reasons, we rejected the possibility that denitrification enzymes could conserve energy.

Given that it is unlikely that denitrifying enzymes conserve energy, the efficiency of aerobic respiration must be lower to account for the observations. If the SOx system directly donates electrons to soluble cytochrome c (Dahl et al., 2008), this would reduce the efficiency by bypassing the energy conserving complex III. However, this would require that complex III operates in both the forward and reverse direction. This occurs in the autotroph Acidithiobacillus ferrooxidans, but it possesses one dedicated complex for each direction (Bruscella et al., 2007). Given that S. denitrificans has only one copy of complex III, we rejected this possibility in the model.

The only plausible explanation remaining to explain reduced efficiency in aerobic res- 
piration was therefore during the oxygen reduction step. The complex predicted to catalyze aerobic respiration in S. denitrificans is a cbb $_{3}$-type cytochrome oxidase (Sievert et al., 2008), which has a high affinity for oxygen and may be specialized for low oxygen tensions (Preisig et al., 1996; Morris and Schmidt, 2013). While this complex is capable of proton transport, it cannot pump protons effectively against membrane potentials typically encountered in living cells (Han et al., 2011; Rauhamäki et al., 2012a; Murali et al., 2012; Rauhamäki et al., 2012b; Rauhamäki and Wikström, 2014). Therefore, under physiological conditions, it is possible that $S$. denitrificans' cbb $_{3}$ cytochrome oxidase does not pump protons.

However, even without pumping protons, aerobic growth yields in silico remained much higher than denitrification. This is because protons for oxygen reduction typically come from the cytoplasm, contributing to the proton-motive force (Nicholls and Ferguson, 2013). However, physiological data has consistently shown that aerobic respiration in $S$. denitrificans yields only slightly higher amounts of biomass versus denitrification (TimmerTen Hoor (1981); Table 4.2). Given these data, we posit that the source of protons for oxygen reduction come from the periplasm instead of the cytoplasm. With this hypothetical mechanism in place (Fig 4-3), the model reproduced experimental growth yield data satisfactorily, and it was considered to be complete.

\subsection{Discussion:}

Although redox reactions that support autotrophy in Epsilonproteobacteria are now well known, the underlying biochemistry remains poorly understood. In this study, we used theoretical and experimental techniques to infer likely mechanisms supporting the autotrophic growth of Sulfurimonas denitrificans. A computational approach using the modelSEED database was first employed to predict a core metabolic network. However, because of a lack of knowledge about the pathways associated with autotrophy, this network initially did not reproduce the experimentally determined physiology and growth yields of S. denitrificans.

To identify likely mechanisms and their efficiency of energy conservation, data from related organisms were used to develop hypothetical mechanisms. For each model iteration, we simulated growth yields in silico using flux balance analysis (FBA) which accurately reproduces both overall efficiency as well as the ratio between growth yields on different 


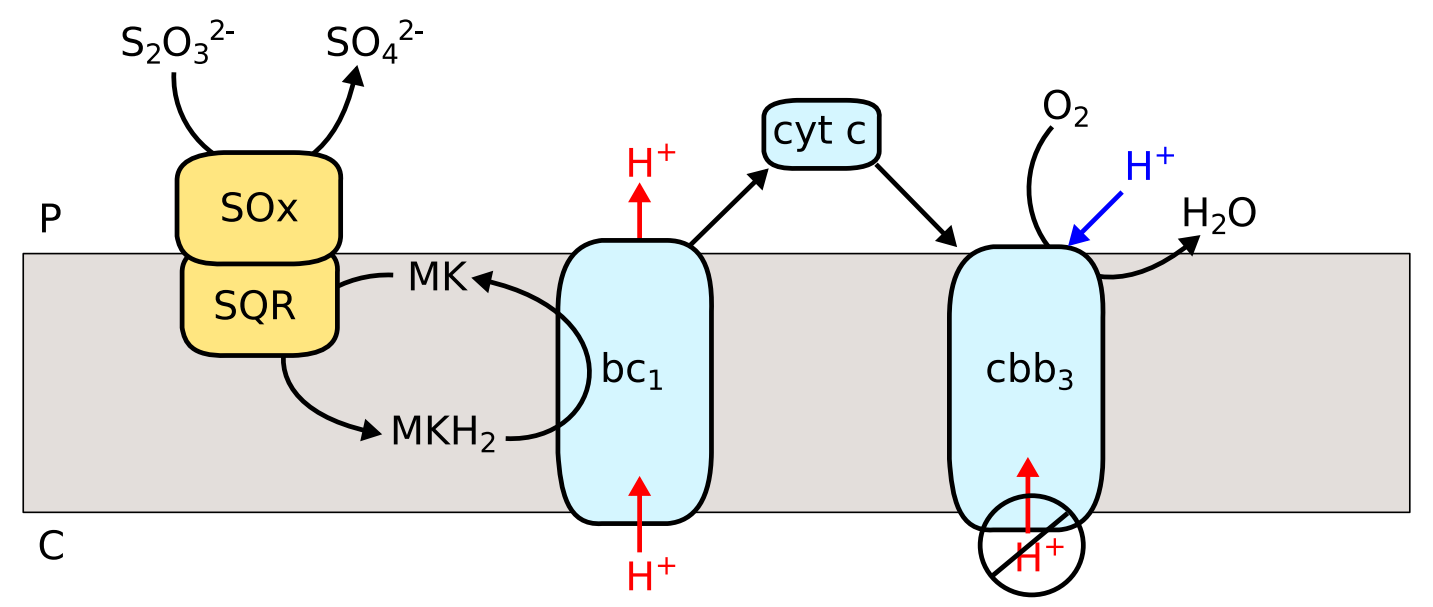

Figure 4-3: Hypothetical model of electron transport and energy conservation during aerobic respiration coupled to sulfur oxidation in Sulfurimonas denitrificans. Black arrows indicate electron transport, red arrows contributions to the proton-motive force and blue arrows are chemical protons used during reduction of $\mathrm{O}_{2}$ to $\mathrm{H}_{2} \mathrm{O}$. No protons are predicted to be pumped by the cbb3 cytochrome oxidase, as indicated in the diagram see text for explanation. $\mathrm{SOx}=\mathrm{SOx}$ sulfur oxidation complex; $\mathrm{SQR}=$ Sulfide:quinone oxidoreductase; $\mathrm{bc}_{1}=$ Complex III; MK $/ \mathrm{MKH}_{2}=$ Oxidized/reduced menquinone; cyt $\mathrm{c}=$ Soluble cytochrome; $\mathrm{cbb}_{3}=$ high-affinity cytochrome $\mathrm{c}$ oxidase. $\mathrm{P}=$ Periplasm; $\mathrm{C}=$ Cytoplasm.

energy sources. Overall efficiency is the proportion of electrons derived from substrate oxidation used to fix $\mathrm{CO}_{2}$, equivalent to (1-y) in Klatt and Polerecky (2015). It is possible to have a model where one, but not both of these parameters is consistent with experimental data. Therefore, if the in silico model reproduces both the correct ratio between different growth substrates and overall growth yields in vivo, this strongly indicates it is realistic.

Using this logic, we produced a best-guess for the core metabolism of S. denitrificans that reproduces observed the physiology and growth yield under different growth conditions. Below, we discuss remaining uncertainties, biochemical experiments to test hypotheses, and the potential implications for autotrophic metabolism in Epsilonproteobacteria.

\subsubsection{Thiosulfate- and Hydrogen-Dependent Denitrification:}

S. denitrificans was isolated with thiosulfate and nitrate (Timmer-Ten Hoor, 1975). However, in silico growth was initially not possible with these substrates. This was because the mechanism assumed for the SOx sulfur oxidation system in S. denitrificans donates electrons to soluble cytochrome c (Friedrich et al., 2001; Dahl et al., 2008). Therefore, electrons 
from sulfur oxidation could not be (exergonically) transferred to the quinone-dependent Nap nitrate reductase complex. Subsequent denitrification enzymes accept electrons from cytochrome c and could therefore be directly linked to the canonical SOx system. However, since these complexes are non-electrogenic (Chen and Strous, 2013), thiosulfate-dependent denitrification could not generate bioavailable energy unless thiosulfate oxidation donated electrons at the level of the membrane quinone pool. Therefore, we incorporated such a hypothetical mechanism in this model (Fig 4-1a).

Biochemical data from the related genus Sulfurovum are consistent with a membranebound SOx complex (Yamamoto et al., 2010). In Sulfurovum NBC37-1, both membranebound and soluble protein fractions are necessary for nitrate-dependent thiosulfate oxidation. Similar data have been reported in the more distantly related alphaproteobacterium Starkeya novella (Oh and Suzuki, 1977a,b; Kappler et al., 2001). Since such a process requires a membrane anchor, what protein(s) serve this role in S. denitrificans is an important unanswered question. Yamamoto et al. (2010) identified several proteins with thiosulfateoxidizing activity that have putative membrane-binding motifs. These included a SoxH protein, a cytochrome c, and a sulfide:quinone oxidoreductase (SQR)-like protein. SQR proteins are membrane-bound, and capable of sulfide-dependent quinone reduction in $S$. denitrificans and other organisms (Griesbeck et al., 2002; Han and Perner, 2016). Consistent with SQR's possible role in thiosulfate oxidation (Yamamoto et al., 2010), RT-qPCR shows that one putative SQR in S. denitrificans (Suden_0619) is highly expressed in the absence of sulfide (McNichol and Sievert, unpublished).

In addition to circumstantial support from the literature, this mechanism closely reproduces experimental growth yields of $S$. denitrificans (Table 4.2) and explains the large difference between hydrogen and thiosulfate oxidation. As has been observed by ourselves and others (Table 4.2) (Han and Perner, 2014), growth yields per mole nitrate are much higher with hydrogen as an electron donor - consistent with a more negative redox midpoint potential for hydrogen vs. sulfur oxidation (Canfield et al., 2005). A membrane-spanning hydrogenase similar to that found in Wolinella succinogenes is found in S. denitrificans (Gross et al., 1998; Sievert et al., 2008) and because of its transmembrane subunits and cytoplasmic quinone binding site, this hydrogenase abstracts protons from the cytoplasm during hydrogen-dependent reduction of quinone. When the quinol thus produced is reoxidized (e.g. by Nap or cytochrome bc 1 ), protons are released into the periplasm (Fig 4-1b), 
generating a proton-motive force via a redox-loop mechanism (Simon et al., 2008).

In contrast, periplasmic sulfur oxidizing enzymes likely do not span the membrane (Marcia et al., 2009; Yamamoto et al., 2010). Therefore, thiosulfate-dependent quinone reduction is probably electroneutral, although a proton-motive force can still be generated during denitrification with thiosulfate via complex III (cytochrome $\left.b_{1}\right)$. This electrogenic complex oxidizes quinol and transfers electrons to soluble cytochrome c, which means electron transport to nitrite, nitric oxide and nitrous oxide is overall an electrogenic process. Complex III will also conserve energy with hydrogen oxidation, but this is in addition to the energy from the aforementioned redox loop. With these mechanisms in place, we found the model closely mirrors physiological growth yields (Table 4.2).

By defining such hypotheses, we can also propose direct experimental tests (Table 4.3). For example, thiosulfate-dependent denitrification should depend wholly on complex III for energy conservation if our model is correct. Therefore, inhibition of complex III should abolish growth in thiosulfate-nitrate medium. While nitrate reduction could still occur, it would not generate energy and denitrification could not proceed beyond nitrite (which may accumulate in the medium). In contrast, inhibiting complex III in hydrogen/nitrate medium would reduce, but not completely inhibit growth, due to the fact that the redoxloop mechanism discussed above is not dependent on complex III. In addition, thiosulfatedependent quinone reduction could also be directly assayed, and gene knockouts used to determine which membrane proteins are essential for this process. 
Table 4.3: Hypothetical biochemical mechanisms and experimental tests.

\begin{tabular}{|c|c|c|}
\hline Hypothesis & Predictions of hypothesis & Experimental test and expected result \\
\hline $\begin{array}{l}\text { SOx complex is attached to } \\
\text { membrane and transfers } \\
\text { electrons to quinone pool }\end{array}$ & 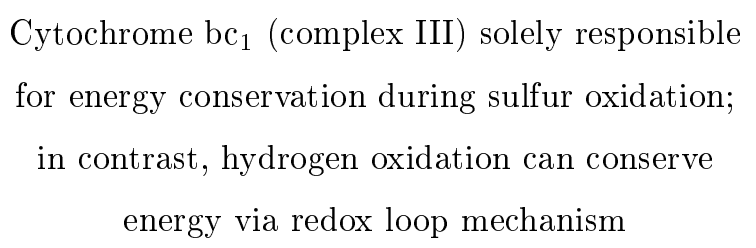 & $\begin{array}{l}\text { Knock out or inhibit complex III: Thiosulfate-nitrate } \\
\text { dependent growth should stop; hydrogen-nitrate dependent } \\
\text { growth should continue with lower growth yield. Nitrite } \\
\text { accumulates in medium }\end{array}$ \\
\hline $\begin{array}{l}\text { cbb }_{3} \text { cytochrome c oxidase } \\
\text { is electroneutral }\end{array}$ & $\begin{array}{l}\text { Energy conservation is solely dependent on } \\
\text { complex III; protons for oxygen reduction are } \\
\text { abstracted from periplasm }\end{array}$ & $\begin{array}{l}\text { Inhibit and bypass cytochrome c oxidase: } \mathrm{Use}^{-} \mathrm{CN}^{-} \text {to inhibit } \\
\mathrm{cbb}_{3} \text { and grow cells with artificial electron acceptor for } \\
\text { cytochrome c (ferricyanide). Should give growth yields } \\
\text { identical to that with } \mathrm{O}_{2} \text {; Test which side protons are } \\
\text { abstracted from during oxygen reduction in biochemical assay }\end{array}$ \\
\hline $\begin{array}{l}\text { NADH:ubiquinone } \\
\text { oxidoreductase is } \\
\text { responsible for } \mathrm{NAD}(\mathrm{P})+ \\
\text { and ferredoxin reduction }\end{array}$ & $\begin{array}{l}\text { Complex donates electrons to both } \mathrm{NAD}(\mathrm{P})+ \\
\text { and ferredoxin during reverse electron transport }\end{array}$ & $\begin{array}{l}\text { Use inverted vesicles with complex I and ATPase: Reduction of } \\
\text { ferredoxin and } \mathrm{NAD}(\mathrm{P})+\text { should be possible with ATP present } \\
\text { to generate pmf }\end{array}$ \\
\hline
\end{tabular}




\subsubsection{Aerobic Respiration vs. Denitrification:}

The ability of $S$. denitrificans to grow microaerobically was established shortly after its isolation (Timmer-Ten Hoor, 1975, 1981). While S. denitrificans can tolerate only low levels of oxygen, microaerobic respiration with thiosulfate yields $\approx 60 \%$ more biomass than with thiosulfate/nitrate (Table 4.2; Timmer-Ten Hoor (1981)). In our model, we initially considered that the high-affinity cytochrome $\mathrm{c}$ oxidase $\left(\mathrm{cbb}_{3}\right.$; complex IV) operates with a stoichiometry typical of low-affinity forms of this enzyme (i.e. aa 3 ). Since the low-affinity complex IV produces a proton motive force via both proton pumping and consumption of cytoplasmic protons during oxygen reduction to water, it translocates more protons than complex III $(2 \mathrm{H}+/ \mathrm{e}-$ vs. $1 \mathrm{H}+/ \mathrm{e}-$ for complex III) (Nicholls and Ferguson, 2013). This presented a problem - both overall growth efficiency and the ratio of growth yield between aerobic and denitrifying conditions were far higher in the model than observed by Timmer-Ten Hoor (1981) and ourselves (Table 4.2). While the periplasmic-facing Nap nitrate reductase complex might conserve energy via a mechanism similar to complex III (Kern and Simon, 2008), other evidence argues against this (Brondijk et al., 2004). In addition, if denitrification enzymes were able to conserve energy, it would raise the overall growth yield to levels higher than observed (0.17 modeled vs. 0.10 observed (Klatt and Polerecky, 2015)).

To reconcile our model with experimental data, the most parsimonious explanation is that complex IV in S. denitrificans is completely non-electrogenic. This not only predicts that no protons are pumped by complex IV, but also that protons used in oxygen reduction (so-called chemical protons) are abstracted from the periplasm. While complex IV is nonelectrogenic in our model, aerobic respiration still yields more energy than denitrification. This is because all electrons transferred to oxygen must pass through the electrogenic complex III. In contrast, only $3 / 5$ electrons flow through complex III during denitrification; the remainder are transferred to the non-electrogenic Nap complex.

Supporting the above scheme, experiments show $\mathrm{cbb}_{3}$-type cytochrome c oxidases cannot sustain proton pumping at physiologically-relevant membrane potentials (Han et al., 2011; Rauhamäki et al., 2012a; Murali et al., 2012; Rauhamäki et al., 2012b). The reason behind this surprising observation was discussed by Rauhamäki and Wikström (2014), who noted that high-affinity cytochrome c oxidases such as cbb3 use more free energy in binding their substrate, resulting in less free energy being available to pump protons against a 
membrane gradient (Rauhamäki and Wikström, 2014).

The latter prediction - i.e. that chemical protons for oxygen reduction are abstracted from the periplasm - has not yet been demonstrated for any cytochrome c oxidase. However, it is known that NO reductases obtain the protons for NO reduction from the periplasm, and that they may be the evolutionary precursor of cytochrome c oxidases (Ducluzeau et al., 2009). If this scenario is true, it follows that there may be some extant cytochrome c oxidases that obtain their chemical protons from the periplasm. Indeed, such a discovery would serve as a missing link between $\mathrm{NO}$ and $\mathrm{O}_{2}$ reduction that would reinforce this evolutionary scenario (Mårten Wikström, pers. comm.). As Sievert et al. (2008) noted, S. denitrificans

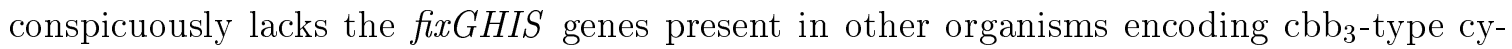
tochrome oxidases, possibly suggesting a different function in these Epsilonproteobacteria. Ultimately, this hypothesis must be tested with biochemical assays (Table 4.3), which would unambiguously establish the source of the protons during oxygen reduction.

A simpler, though less conclusive test would be to compare growth yields of $S$. denitrificans and Sulfurimonas autotrophica (Inagaki et al., 2003). Since the genome of $S$. autotrophica (Sikorski et al., 2010) appears to possess a canonical low-affinity cytochrome oxidase, the entire respiratory electron transport chain should pump 3 protons per electron reduced $(3 \mathrm{H}+/ \mathrm{e}-)$ under high oxygen tensions. In contrast, we predict that the obligately microaerobic S. denitrificans can only pump $1 \mathrm{H}+/ \mathrm{e}-$ in total during thiosulfate oxidation with oxygen as an electron acceptor. Therefore, the yield of S. autotrophica under aerobic conditions should be $\approx 3 \mathrm{x}$ greater than $S$. denitrificans grown under microaerobic conditions.

\subsubsection{Reverse Electron Transport:}

Another pathway essential to the growth of $S$. denitrificans is the autotrophic fixation of $\mathrm{CO}_{2}$ via the rTCA cycle (Hügler et al., 2005). Reduced ferredoxin is required for reductive carboxylation of succinyl-CoA to alpha-ketoglutarate and acetyl-CoA to pyruvate in the rTCA cycle (Evans et al., 1966). However, how reduced ferredoxin is produced in autotrophic Epsilonproteobacteria is unknown (Marshall et al., 2012). Sievert et al. (2008) suggested that a hydrogen-dependent electron bifurcating complex could generate both $\mathrm{NAD}(\mathrm{P}) \mathrm{H}$ and reduced ferredoxin. However, since this mechanism depends on hydrogen, it cannot reduce ferredoxin with reduced sulfur compounds as an electron donor. No known electron- 
bifurcating complexes are present in the S. denitrificans genome (Marshall et al., 2012), nor is there evidence for complexes coupling endergonic ferredoxin reduction to the proton motive force (e.g. Tremblay et al., 2013).

A biochemical study of the heterotrophic epsilonproteobacterium Campylobacter jejuni has revealed a potential mechanism for ferredoxin production in autotrophic Epsilonproteobacteria. Weerakoon and Olson (2008) report the surprising observation that NADH: ubiquinone oxidoreductase (complex I) in C. jejuni does not accept electrons from NADH; rather, reduced flavodoxin serves as the physiological electron donor. Since reduced flavodoxin can have a similar redox midpoint potential to reduced ferredoxin, such a non-canonical complex I run in reverse may produce reduced ferredoxin for the rTCA cycle. Weerakoon and Olson (2008) also showed that this unusual mechanism is due to differences in the NuoE/F subunits that interact with NADH in canonical complex I. Interestingly, they note that $S$. denitrificans and other autotrophic Epsilonproteobacteria also possess non-canonical NuoEF subunits. So far, this different NuoEF composition has also been observed in all genomes of Sulfurimonas and Sulfurovum sequenced to date (Nakagawa et al., 2007; Sievert et al., 2008; Sikorski et al., 2010; Grote et al., 2012; Cai et al., 2014)(NZ_CP011308).

Considering these striking and evolutionarily conserved difference in complex I in Sulfurimonas/Sulfurovum, it appears possible that the S. denitrificans complex I is capable of ferredoxin reduction during reverse electron transport. However, $\mathrm{NAD}(\mathrm{P}) \mathrm{H}$ is also needed as a reductant for the rTCA cycle. In Hydrogenobacter thermophilus, a plant-like ferredoxin-NADP ${ }^{+}$reductase transfers electrons from ferredoxin to NADPH (Ikeda et al., 2009), whereas in Nitrospina gracilis, genomic evidence suggests two distinct complexes may each be specialized for either $\mathrm{NAD}(\mathrm{P}) \mathrm{H}$ or ferredoxin reduction (Lücker et al., 2013). However, there is no evidence for either of these mechanisms in S. denitrificans.

It was therefore proposed that both $\mathrm{NAD}(\mathrm{P}) \mathrm{H}$ and ferredoxin are produced simultaneously via complex I. In this hypothetical reaction, 4 electrons derived from the oxidation of two menaquinol molecules reduce one molecule of $\mathrm{NAD}(\mathrm{P})^{+}$and two molecules of ferredoxin via an electron-bifurcation mechanism (Fig 4-2). This endergonic reaction is driven by the transport of eight protons from the periplasm into the cytoplasm. A similar mechanism has been proposed in anammox bacteria to produce both NADH and formate simultaneously (Kartal et al., 2012).

Several observations provide circumstantial evidence that this hypothesis may be cor- 
rect. Firstly, as mentioned above, the unusual NuoF protein described above is highly conserved in all Sulfurimonas and Sulfurovum genomes $(\geq 66 \%$ protein identity and complete coverage to $S$. denitrificans NuoF). Secondly, this protein is larger than its counterpart in $C$. jejuni (677 vs. 230 aa) or E. coli K-12 NuoF (445 aa). Based on the Pfam database (Finn et al., 2016), the NuoF in $S$. denitrificans is predicted to have both a complete canonical NuoF domain (PF07992.11) plus two additional domains. The first (PF10589.6) is annotated as NAD+-binding domain, whereas the second (PF14691.3) is annotated as a $4 \mathrm{Fe}-4 \mathrm{~S}$ binding domain which could conceivably bind a soluble ferredoxin. In addition, the NuoG protein, the next step in electron transfer from NADH to quinone after NuoEF (Nicholls and Ferguson, 2013), is duplicated in S. denitrificans. This duplication, which is also present all of the genomes discussed above, is also consistent with this mechanism since it could provide two protein wires for electron bifurcation from menaquinol.

It was also observed that NuoF in $S$. denitrificans is homologous to $\mathrm{NfnB}$ sequences from several organisms (Clostridium kluyveri, Thermotoga maritima, Moorela thermoacetica). As demonstrated biochemically, the $\mathrm{NfnB}$ protein is part of a heterodimeric complex that accepts electrons from NADH and ferredoxin during electron bifurcation that produces NADPH (Demmer et al., 2015). Since our mechanism is essentially the reverse of the NfnAB reaction, sequence homology provides circumstantial support for an analogous function.

The ability to use Complex I for reverse electron transport would have several advantages for S. denitrificans. Firstly, since it can operate with any electron donor, growth is possible with both hydrogen and thiosulfate. Secondly, since it is dependent on the proton motive force, it is directly linked to (and should be regulated by) energy generating processes. Finally, it provides both reductants in a 1:1 ratio, meaning their production will always be linked and balanced. This 1:1 ratio may be important, since the rTCA cycle uses ferredoxin and $\mathrm{NAD}(\mathrm{P}) \mathrm{H}$ in a 1:1 ratio if electrons for fumarate reduction come from the membrane quinol pool (Sievert et al., 2008).

While 1:1 production of $\mathrm{NAD}(\mathrm{P}) \mathrm{H}$ and ferredoxin would be advantageous for the rTCA cycle, it could also pose problems to $S$. denitrificans. For example, NAD $(\mathrm{P}) \mathrm{H}$ is used for many anabolic processes (i.e. lipid synthesis), but in the model reduced ferredoxin was not initially needed for reactions outside the rTCA cycle. Indeed, growth was at first not possible without inclusion of a reaction for a ferredoxin-dependent glutamate synthase in the model (Kameya et al. (2007); Suden_1176). By providing a sink for ferredoxin in an essential 
nitrogen assimilation step, several biosynthetic pathways (i.e. amino acid synthesis and nucleotide synthesis) were ultimately dependent on ferredoxin as a reductant, and growth could occur.

However, a sink for "excess" ferredoxin would not be needed if interconversion between ferredoxin and $\mathrm{NAD}(\mathrm{P}) \mathrm{H}$ was possible. Indeed, Maurice et al. (2007) describe an enzyme with such properties (FqrB) in Campylobacter jejuni. If the homologous protein (Suden_0165) has the same function in S. denitrificans, it could serve a redox-balancing role during growth. This opens the possibility that complex I in S. denitrificans functions only as a ferredoxin:menaquinone reductase with $\mathrm{NAD}(\mathrm{P}) \mathrm{H}$ for biosynthesis produced via FqrB. However, flavodoxin oxidation occurs in C. jejuni with a much smaller NuoF subunit and without a duplicated NuoG. Therefore, it appears unlikely that the large NuoF subunit in $S$. denitrificans only reduces ferredoxin; however, only biochemical experiments will be able to resolve this uncertainty.

Several experiments are possible. Testing whether or not ferredoxin:NADP + reductase activity is present in $S$. denitrificans would indicate whether or not ferredoxin reduction by complex I would be sufficient to account for growth. However, a more direct test would be to determine the biochemical potential of complex I in S. denitrificans. For example, inside-out vesicles should couple ATP hydrolysis to the reduction of ferredoxin and NAD $(\mathrm{P}) \mathrm{H}$ if this model is correct (Table 4.3). Such experiments would also clarify whether both substrates are produced simultaneously and whether the stoichiometry predicted here is accurate.

\subsection{Conclusions:}

Our model used a combination of theoretical modeling and growth experiments to infer necessary biochemical mechanisms that support autotrophy in Sulfurimonas denitrificans. Not only are mechanisms parsimonious and consistent with experimental data, they provide a theoretical framework allowing the design of specific biochemical experiments to test hypothetical mechanisms (Table 4.3). Such direct biochemical data will provide fundamental information regarding the core metabolism of autotrophic Epsilonproteobacteria and represent a first step towards quantitative modeling of their productivity in the natural environment. In addition, studying core energy-converting enzymes may help understand the evolution of autotrophy in Epsilonproteobacteria. For example, deep-branching autotrophic 
Epsilonproteobacteria such as Caminibacter mediatlanticus and Nautilia profundicola possess a complex I that completely lacks NuoEFG subunits (Weerakoon and Olson, 2008; Campbell et al., 2009). Such a truncated complex I resembles so-called energy-converting hydrogenases (Ech) that are thought to be the evolutionary precursors of complex I (Hedderich, 2004). Since this form of complex I is only found in hydrogen-dependent and oxygen-sensitive taxa, it may represent an ancestral form of this core bioenergetic complex. In contrast, in $S$. denitrificans and related Epsilonproteobacteria that are not hydrogen-dependent and tolerant to oxygen, complex I is larger and contains additional subunits. Therefore, the apparent evolutionary changes in complex I in autotrophic Epsilonproteobacteria may reflect their adaptive radiation into oxic, hydrogen-poor niches that became available during the rise of atmospheric oxygen on ancient Earth.

\subsection{Acknowledgements:}

Many thanks are due to Jörg Simon, Mårten Wikström and Martin Klötz for helpful discussions regarding bioenergetics. Thanks also to Florian Götz and Dali Smolsky for help with chemostat cultivation and to Mak Saito for lending his lab's mass-flow controller. 


\section{Chapter 5}

\section{Conclusions and Future Work:}

\subsection{Hydrothermal Fluid Incubations:}

Shortly after the discovery of deep-sea hydrothermal vents, Karl, Wirsen and Jannasch (1980) opined that:

"In view of the complexity of the entire vent system and the limited amount of sampling possible, a useful quantification of deep-sea primary production is quite out of reach at this time."

Although written nearly forty years ago, their words still ring true despite major advances in understanding free-living microbial communities at deep-sea hydrothermal vents. As discussed above, the microbial communities' taxonomic identity was first revealed in the mid to late 1990s (Haddad et al., 1995; Polz and Cavanaugh, 1995) and isolates were obtained shortly thereafter (Campbell et al., 2001; Takai et al., 2003). In turn, novel sequencing technologies have revealed complex population structures (Huber et al., 2007) and more advanced technologies can now identify active metabolic pathways in situ (Urich et al., 2014; Fortunato and Huber, 2016).

These studies, without a doubt, have transformed how we view hydrothermal vent microbial communities. Nevertheless, they cannot quantitatively constrain the influence of these organisms' metabolism on biogeochemical cycles; at best, they identify active organisms and their potential metabolic pathways. Therefore, the importance of the biogeochemical processes catalyzed by these natural microbial communities remains fundamentally 
uncertain (Sievert and Vetriani, 2012).

The experiments discussed in Chapters 2 and 3 make several contributions towards understanding the activity of hydrothermal vent microbial communities in situ. Firstly, they provide compelling experimental evidence that autotrophic Epsilonproteobacteria dominate subseafloor biogeochemical processes and offer important insights into their physiological diversity. Secondly, by combining primary productivity data with electron acceptor consumption, the growth efficiency of natural communities was determined. Using these data, it was shown that $\mathrm{pO}_{2}$ and high temperatures reduced the overall community growth efficiency. High temperatures and $\mathrm{pO}_{2}$ also markedly influenced fine-scale population structure of Sulfurimonas and Thioreductor OTUs. Another important result of determining growth efficiency was that it allowed in situ primary productivity, biomass standing stock, and residence time to be determined - all parameters that were previously poorly constrained due to a lack of experimental data. These data also provide insights into the poorly described dynamics of the subseafloor ecosystem, indicating that subseafloor microbial biomass turns over on a similar or faster timescale compared to surface ocean phototrophs (Falkowski and Raven, 2013). In summary, these incubations have provided what some might consider "...a useful quantification of deep-sea primary productivity" (Karl et al., 1980), and constitute an important first step to assess the role of deep-sea vent ecosystems in global biogeochemical cycles.

In the future, primary productivity at other hydrothermal vents could be estimated using the theoretical framework presented here. Since vent microbial communities are often dominated by Epsilonproteobacteria (Engel et al., 2004; Nakagawa et al., 2005b; Moussard et al., 2006), growth efficiencies presented in Chapter 3 may be broadly applicable. Therefore, only fluid flux, end-member and low-temperature fluid chemistry would be necessary to reconstruct the potential productivity for a given ecosystem. It would also be interesting to apply the incubation techniques discussed in Chapters 2 and 3 to different environments. For example, preliminary evidence presented in Chapter 3 suggests that microbial communities at $\mathrm{Crab} S p a$ have lower growth efficiencies at $50^{\circ} \mathrm{C}$. However, this may be because the resident microbial community is not adapted to higher temperatures. Would different results be obtained from a hydrothermal vent with a $50^{\circ} \mathrm{C}$ exit temperature? The incubation approach could also be applied to investigate biological rates and growth efficiency of microbial communities present in hydrothermal vent plumes (Anantharaman et al., 2016). 
The chemosynthetic growth efficiency (CGE) parameter that underlies primary productivity estimates could also be investigated further. CGE should differ from organism to organism depending on the properties of their energy-conserving protein complexes. For example, the strain Sulfurimonas autotrophica that tolerates higher levels of $\mathrm{O}_{2}$ appears to have an inherently higher growth efficiency compared to microaerophiles such as S. denitrificans (see preceding discussion in Chapter 4). While maximum growth efficiencies observed during incubations are consistent with values from microaerophiles such as $S$. denitrificans (Klatt and Polerecky, 2015), higher levels of oxygen reduced efficiencies substantially. This is counter-intuitive, considering the observed potential for higher growth efficiencies under oxic conditions in isolates such as $S$. autotrophica. The observation of such low CGE was explained by considering that some carbon-fixing rTCA enzymes are inherently sensitive to oxygen (Imlay, 2006). While this suggests an explanation for lower CGE under oxic conditions, it does not address the inverse question: i.e. why did we not observe higher CGE under oxic conditions as in pure cultures of $S$. autotrophica? Since CGE could be up to 3-fold higher for such an organism, their presence in situ would increase the overall productivity of the subseafloor biosphere substantially if they were abundant. Were these organisms present in incubations? If so, why did the community growth efficiency remain so low under oxic conditions? Was their more efficient metabolism counter-balanced by the inefficient metabolism of microaerophiles struggling to cope with high $\mathrm{O}_{2}$ levels? Similarly, Epsilonproteobacteria that could use $\mathrm{H}_{2}$ as an electron donor coupled to respiration of $\mathrm{O}_{2}$ with a low-affinity cytochrome c oxidase would also have much higher growth efficiencies. Do such "knallgas" Epsilonproteobacteria exist? How abundant are they in natural communities?

In addition to CGE, other parameters warrant further investigation. In Chapter 3, subseafloor biomass standing stock was also estimated using two essential pieces of information: per-cell rates of nitrate and oxygen reduction, and carbon content per cell. Although per-cell rates were well-constrained across a variety of conditions in Chapter 2, it seems likely that rates represent an upper limit; natural communities that are substrate-limited probably have slower per-cell rates. Likewise, the carbon content of individual cells in the natural environment is very uncertain. In Chapter 3, a conservative estimate of $173 \mathrm{fg}$ carbon per cell was used for subseafloor standing stock determinations based on an average cell size of $0.8 \mathrm{um}^{3}$ derived from cells measured during NanoSIMS analysis. However, natural environmental samples appear to contain cells with much larger biovolumes than those 
found at the end of incubations. If the carbon density of these larger cells were similar to smaller cells, it would increase the subseafloor standing stock estimate. Investigating the cell carbon density of natural populations would be straightforward - large quantities of biomass from the subseafloor could be filtered, weighed and divided by cell abundances to derive a robust estimate of per-cell carbon content. However, estimating per-cell rates of natural communities without "bottle effects" is considerably more challenging. Although Chapter 2 discusses methods that permit incubations under in situ conditions, there were clear biases. For example, in the natural community, sequences of the genera Arcobacter, Sulfurovum and Sulfurimonas are all abundant (Sievert, unpublished). However, Sulfurimonas was dominant under all incubation conditions at $24^{\circ} \mathrm{C}$. Similarly, Thioreductor form only a small portion of the background community ( $\approx 2 \%$ of sequences), yet became abundant in some incubations. Did this community shift affect rate measurements? Put another way, would rates have been different if organisms such as Arcobacter and Sulfurovum were more active during incubations? Perhaps even shorter in situ incubations could be carried out that would not bias community structure though measuring rates in biofilm communities (likely the most abundant form of microbial biomass in the subseafloor) is especially challenging and would require new methods development.

Changes in community structure during incubations are also interesting from an ecological perspective. What factors allowed Sulfurimonas and Thioreductor to apparently outcompete other organisms such as Sulfurovum and Arcobacter during incubations? Purely physical/chemical factors could be responsible. For example, organisms such as Arcobacter may be adapted specifically to $\mathrm{O}_{2}-\mathrm{H}_{2} \mathrm{~S}$ interfaces (Wirsen et al., 2002). Since such sharp concentration gradients were not likely present in IGT samplers, this particular ecological niche may not have been available during incubations. In a more homogenous environment, perhaps Arcobacter strains were not able to compete effectively for resources with Sulfurimonas and Thioreductor that may have higher affinity enzymes for limiting electron acceptors such as $\mathrm{NO}_{3}{ }^{-}$and $\mathrm{O}_{2}$. This hypothesis could be directly tested in the future using gradient cultivation techniques.

The differences in community structure between incubations and natural communities could also be related to microbes' lifestyles. As mentioned above, biofilms are likely the dominant growth mode in the natural environment. However, during incubations, regular stirring may have prevented biofilms from forming. If some organisms are preferentially 
found in biofilms, this could have biased the community composition towards non-sessile microorganisms such as Sulfurimonas. This could be one important factor as to why Sulfurovum did not appear active in our incubations, despite being abundant in in situ gene surveys. CARD-FISH probes specific for Sulfurovum were designed during this thesis, which could be used to investigate this hypothesis. Initial observations suggest that Sulfurovum cells derived from natural samples tend to be found as large filaments. Because of their size, it should be relatively straightforward to enrich such organisms through size-selective filtration. Ideally, isolates would be obtained but even metagenomics or single-cell genomics would be useful to investigate the potential genetic differences associated with a sessile lifestyle.

\subsection{Genome Scale Metabolic Modeling:}

In addition to the field component described above, Chapter 4 of this thesis discussed a series of predictions for the biochemical mechanisms that support autotrophic growth in Sulfurimonas denitrificans. Based on physiological data and observations from related organisms, this study provides a theoretical framework to guide future experiments to investigate the core-energy conserving complexes in autotrophic Epsilonproteobacteria. Once the precise biochemical mechanisms are known, it will not only allow for quantitative modeling of these organisms' metabolism but also help understand the evolution and adaptive radiation of Epsilonproteobacteria. As described by Weerakoon and Olson (2008), mesophilic Epsilonproteobacteria have a unique form of complex I in which the NuoEF subunits are non-homologous to other organisms. This observation was used to hypothesize that complex $\mathrm{I}$ is the site of reversed electron transport for producing ferredoxin and NAD $(\mathrm{P}) \mathrm{H}$. Weerakoon and Olson (2008) as well as Campbell et al. (2009) also note that members of the deep-branching Nautiliales encode a yet different form of Complex I that completely lacks the NuoEFG subunits. As discussed in the introduction, genomes of Nautilia profundicola and Caminibacter mediatlanticus (the deepest-branching Epsilonproteobacteria (Zhang and Sievert, 2014)) as well as several Lebetimonas isolates (Meyer and Huber 2013) show that these organisms all possess this incomplete form of complex I as well as "energy-conserving hydrogenases" (Ech; Hedderich, 2004). Ech, which couple hydrogen oxidation to ferredoxin reduction, also lack NuoEFG subunits and are thought to be the evolutionary precursor of 
complex I (Hedderich, 2004). Both these Ech complexes and the incomplete Complex I are absent in Sulfurimonas and related Campylobacterales.

Therefore, it appears that the advent of the NuoEFG subunits and loss of Ech in Sulfurimonas and other related strains was an evolutionarily-significant event. What was the driving force behind this change? Why do Nautilia and Caminibacter encode two subunits with an apparently homologous function in ferredoxin reduction? Since deep-branching Epsilonproteobacteria contain what is thought to be the most ancestral forms of complex I (Esposti, 2016), investigations into the functions of these enzymes in both deep- and shallowbranching Epsilonproteobacteria will shed light on the evolution of this core bioenergetic enzyme complex. As discussed in Chapter 4, this evolutionary change likely has to do with their adaptive radiation from hydrothermal environments into more oxic environments that appeared during the oxygenation of ancient Earth.

As part of these organisms' apparent adaptation to oxic niches, they appear to have gained several functions - the ability to use oxygen, carry out denitrification and oxidize sulfur. These functions all depend on the presence of complex III - a multimeric energyconserving enzyme. Complex III transfers electrons from quinol to soluble cytochrome c, which then donates electrons to cytochrome $\mathrm{c}$ oxidase and most denitrification enzymes. Not only does this allow for the use of more oxidizing substrates as electron donors, its ability to translocate protons is likely essential for conserving energy during denitrification and microaerobic growth. When did this complex emerge in Epsilonproteobacteria? Was it acquired from other organisms or did it evolve indigenously? Similarly, when did sulfur oxidation emerge in these organisms? Currently, there is no evidence that sulfur oxidation occurs in deep-branching lineages of Epsilonproteobacteria; rather, these organisms are dependent on $\mathrm{H}_{2}$ as an electron donor and use sulfur only as an electron acceptor or source for biosynthesis. When and how did sulfur oxidation evolve in these organisms? Some insight into this evolutionary process may be obtained by investigating organisms that appear to have characteristics of 'deep-branching' and 'modern' Epsilonproteobacteria. For example, in the incubations discussed in Chapters 2 and 3, we observed the growth and carbon fixation of Thioreductor-like organisms which are from the deep-branching Nautiliales clade. The only isolate of this genus is an obligate anaerobe dependent on hydrogen as an electron donor, which is consistent with the physiology of deep-branching isolates. Surprisingly, we also observed the growth of such organisms in the absence of hydrogen and in the presence 
of oxygen. This observation suggests that such deep-branching Epsilonproteobacteria may be able to use sulfur as an electron donor. If this inference could be confirmed through isolation of a representative culture, then it would be possible to investigate the responsible pathways and understand how such an organism conserves energy for growth. In turn, these data might point towards a plausible scenario for the emergence of sulfur oxidation within the deep-branching Epsilonproteobacteria.

Although apparently "modern" Epsilonproteobacteria such as Sulfurimonas can use oxygen as a terminal electron acceptor, the modeling approach predicted these organisms'

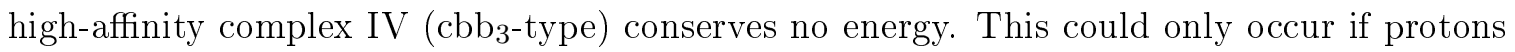
for the reduction of $\mathrm{O}_{2}$ to $\mathrm{H}_{2} \mathrm{O}$ were obtained from the periplasm and if no protons were pumped during $\mathrm{O}_{2}$ reduction. While the latter proposition is reasonable based on current observations (see discussion in Chapter 4), the former would represent a unique discovery. Since the only related proteins that carry out such a process are NO reductases, such a discovery would lend credence to the hypothesis that cytochrome c oxidases evolved from NO reductases. Aside from evolutionary implications, such a non-energy conserving cytochrome c oxidase must also provide some advantage to the autotrophic Epsilonproteobacteria that possess it. Could such a complex be an effective tool to compete for traces of $\mathrm{O}_{2}$ in the natural environment, similar to the Nap complex for nitrate reduction? Put another way, could the disadvantage to an organism in terms of its "inefficiency" be made up for by the higher speed of the enzyme complex? If so, it would provide another example of an adaptation that autotrophic Epsilonproteobacteria use to compete effectively in electron acceptor-limited environments.

\subsection{Ongoing Research:}

The differential response of individual Sulfurimonas OTUs and the activity of Thioreductor-like organisms in the presence of oxygen was a surprising result of the incubations. In order to investigate the genomic basis of this response, I applied to the JGI small-scale microbial/metagenome program and was granted funding to sequence 48 single-cell genomes. This will support the sequencing of six representative single-cell genomes (SAGs) from eight separate groups of Sulfurimonas - those that had a strong positive or negative response to oxygen and/or were very abundant in incubations. By sequencing six closely-related SAGs 
from each group, I am hopeful that a 'consensus genome' can be assembled that represents the metabolic potentials of these different groups. Subsequently, differences in genes/pathways that could account for the observed patterns can be determined.

Environmental sequence data, especially SAGs, may offer important clues towards the putative biochemical underpinnings of these uncultivated strains of autotrophic Epsilonproteobacteria. However, hypothetical mechanisms for any phenomenon and the physiological significance of such differences must ultimately be confirmed experimentally. In my view, combining these modern techniques with classical isolation approaches, physiological experimentation (e.g. in chemostats; (Veldkamp and Jannasch, 1972)), and direct biochemical characterization of putative energy-conserving complexes (e.g. Weerakoon and Olson, 2008; Nicholls and Ferguson, 2013) has great potential to resolve the outstanding uncertainties about the metabolism of autotrophic Epsilonproteobacteria. For example, the use of SAGs can give insight into the metabolic characteristics of uncultivated organisms. Using this information, it may be possible to design a more rational isolation campaign to capture the uncultivated diversity in natural environments. In turn, lab-derived data on mechanisms, physiology, and genetic diversity from such isolates will not only have important implications for understanding natural communities, but also help understand the origin, evolution and diversification of the Epsilonproteobacteria as a whole. 
Appendix A

Supplemental Material for Chapter 3 


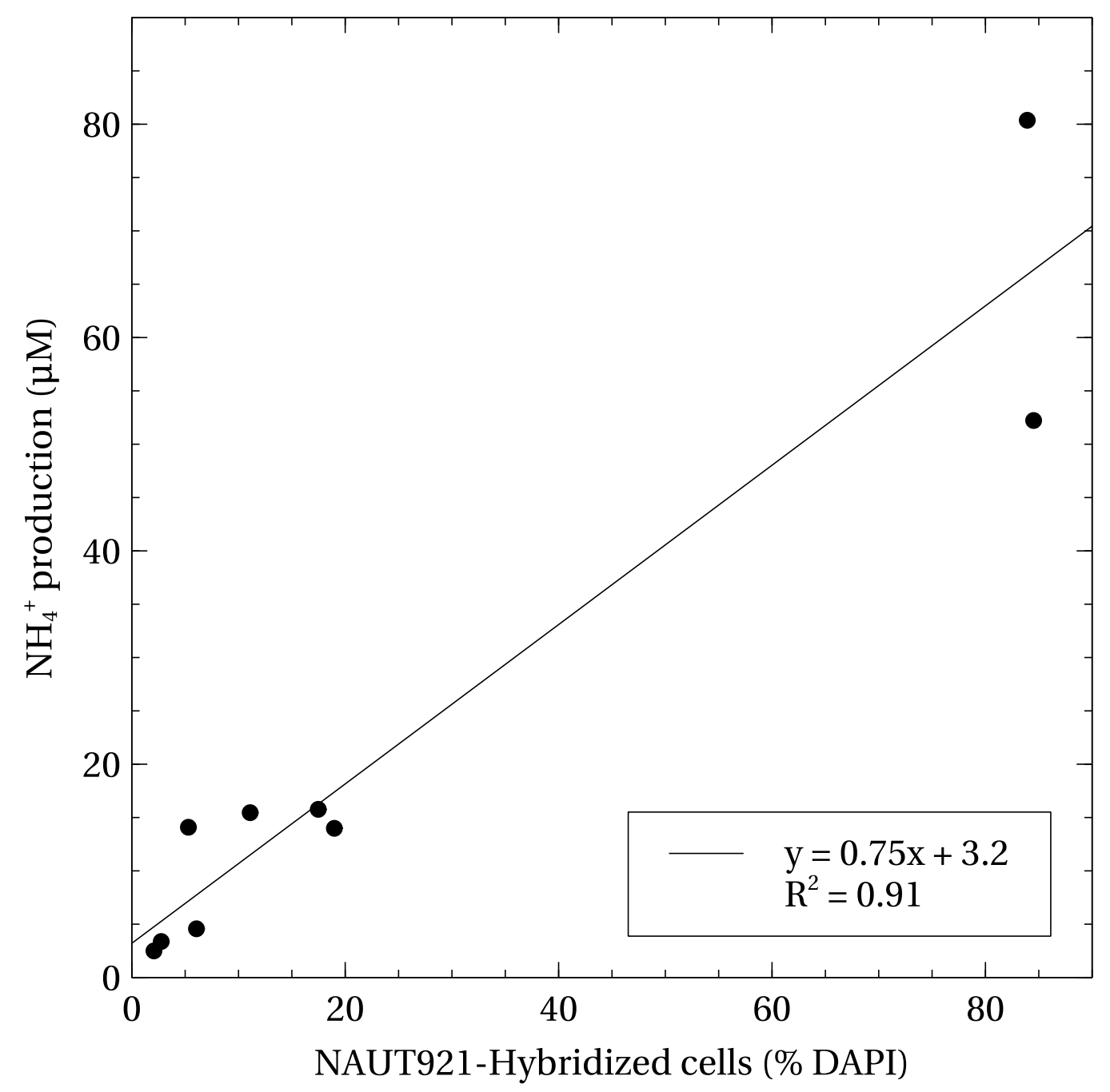

Figure A-1: Correlation between the proportion of cells hybridized to the Nautiliales-specific probe (NAUT921) and ammonium production. Each point represents a separate incubation (biological replicate). 


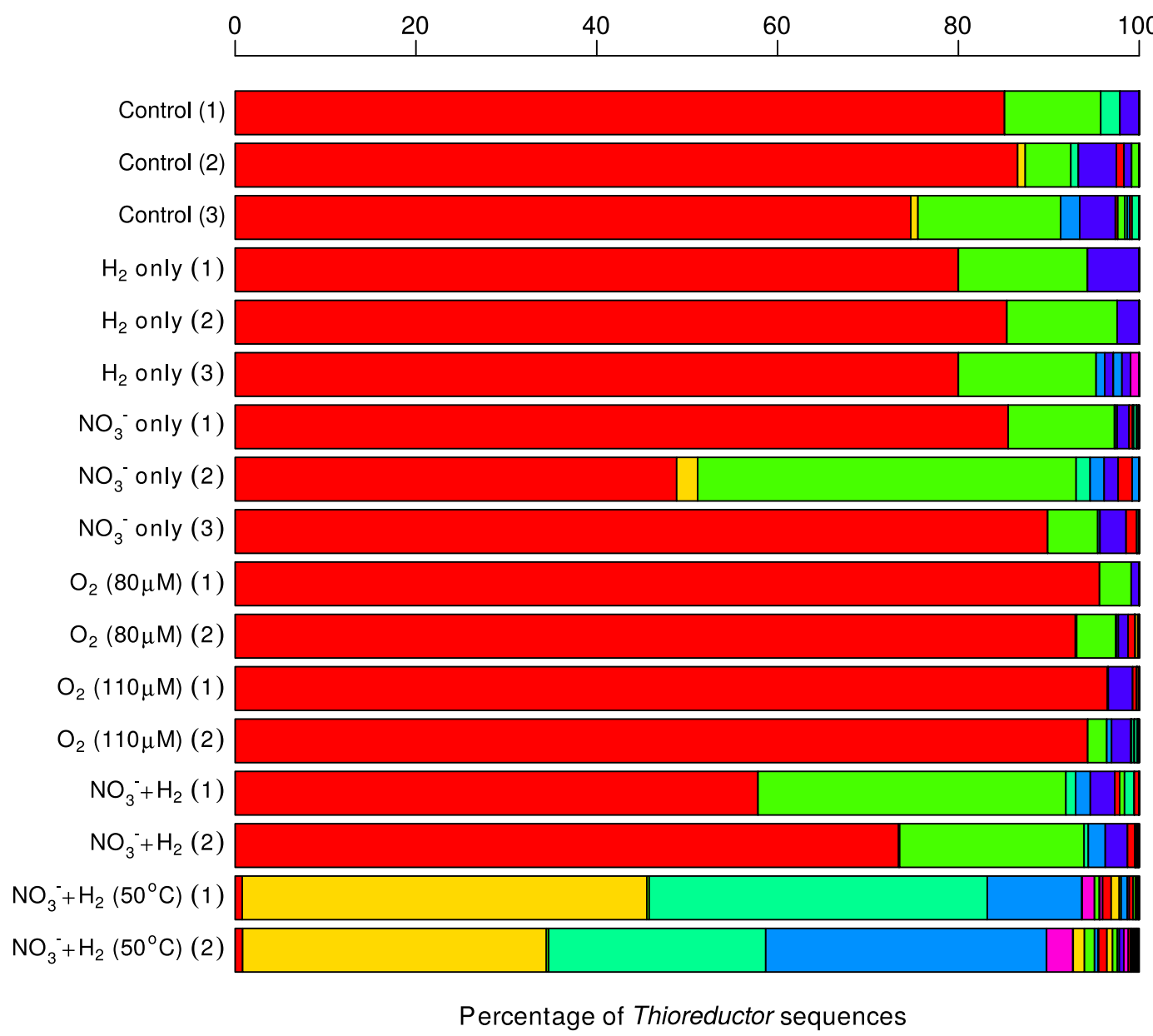

Figure A-2: Thioreductor 97\% OTU composition across different treatment conditions. 


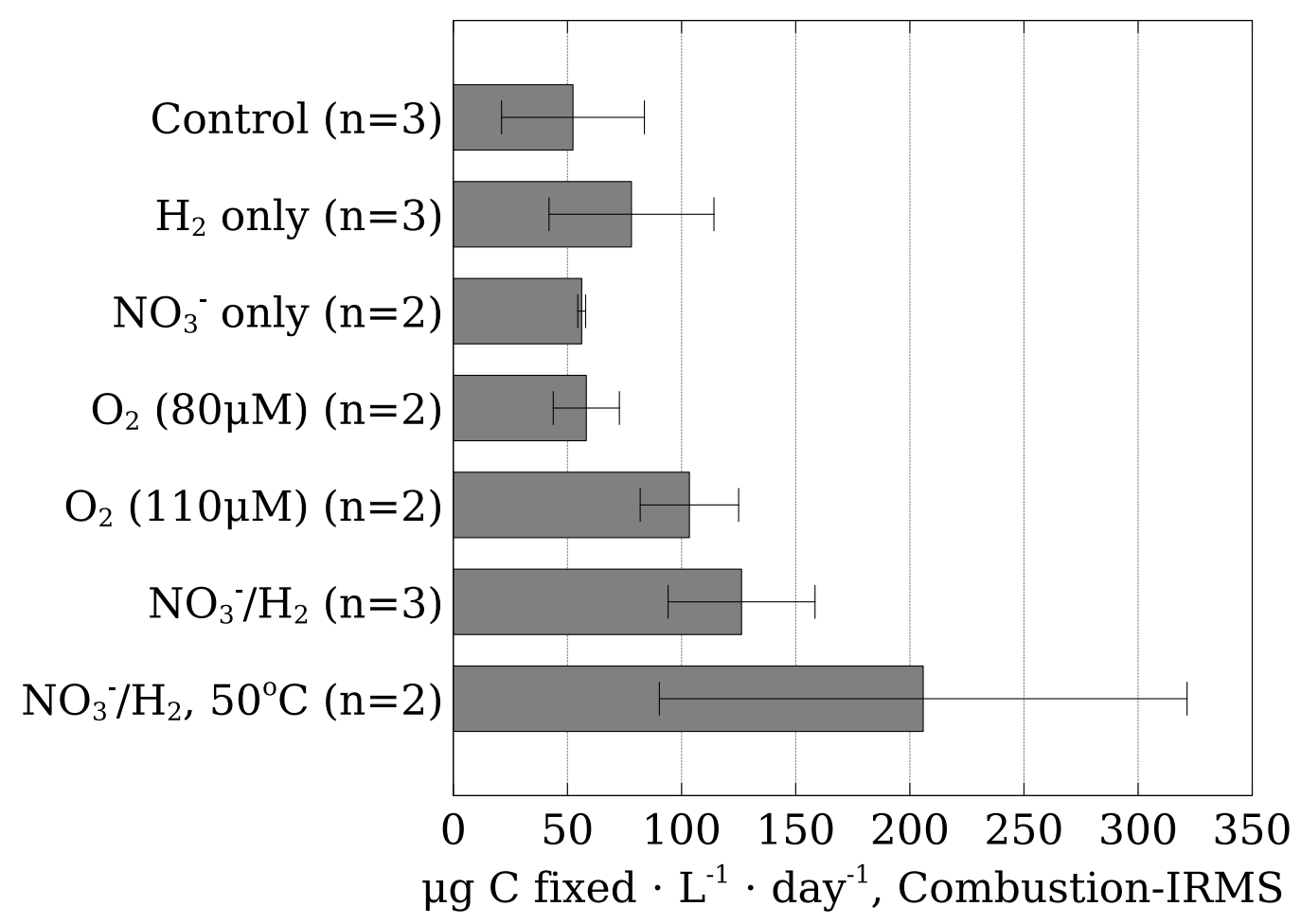

Figure A-3: Absolute carbon fixation rates across different treatment conditions derived by combustion of unfixed bulk microbial biomass. Errors are standard deviations $(n=3)$, or ranges $(n=2)$. 
Table A.1: Proportion of cells affiliated to different taxonomic groups under different incubation conditions as determined by hybridization of specific oligonucleotide probes and signal amplification with catalyzed reporter deposition fluorescence in-situ hybridization (CARDFISH).

\begin{tabular}{|c|c|c|c|c|}
\hline Condition & $\begin{array}{l}\text { Incubation Temperature } \\
\qquad\left({ }^{\circ} \mathrm{C}\right)\end{array}$ & $\begin{array}{l}\text { Sulfurimonas* } \\
\text { (\% DAPI) }\end{array}$ & $\begin{array}{c}\text { Nautiliales }^{* *} \\
\text { (\% DAPI })\end{array}$ & $\begin{array}{c}\text { Gammaproteobacteria }^{* * *} \\
(\% \mathrm{DAPI})\end{array}$ \\
\hline Background (no incubation) & NA & $34.4,30.3$ & $6.8,8.4$ & $4.3,23.8$ \\
\hline Control & 24 & $35.9,68.1,62.2$ & $3.5,4.9,5.8$ & $30.2,13.5,5.2$ \\
\hline $\mathrm{H}_{2}$ addition & 24 & $76.7,74.8,65.8$ & $2.1,3.0,2.8$ & $2.0,7.2,12.8$ \\
\hline $\mathrm{O}_{2}$ addition $(80 \mu \mathrm{M})$ & 24 & $84.1,84.4$ & $5.0,20.0$ & $6.2,2.5$ \\
\hline $\mathrm{O}_{2}$ addition $(110 \mu \mathrm{M})$ & 24 & 74.0 & $16.7,16.7$ & $7.0,1.7$ \\
\hline $\mathrm{H}_{2} / \mathrm{NO}_{3}{ }^{-}$addition & 24 & $85.2,85.9,75.8$ & $11.1,19.0,17.5$ & $2.2,1.8,8.6$ \\
\hline $\mathrm{H}_{2} / \mathrm{NO}_{3}{ }^{-}$addition & 50 & $19.7,14.8$ & $83.9,84.5$ & $11.0,11.3$ \\
\hline
\end{tabular}

* SFMN287 (this study); ** NAUT921 (this study); *** GAM42A (Manz et al., 1992) 
Table A.2: Probe sequences used in this study and predicted coverage based on testprobe (http://www.arb-silva.de/search/testprobe/).

\begin{tabular}{|c|c|c|}
\hline & NAUT921 & SFMN287 \\
\hline Probe sequence & TTGTTCGGGTCCCCGTCT & ATCCTCTCAAACCCGCTA \\
\hline \multirow{3}{*}{ Competitor sequence(s) } & \multirow{3}{*}{ TTGTGCGGGTCCCCGTCT } & ATCCTCTCAAACCCCCTA \\
\hline & & ATCCTCTCAAACCAGCTA \\
\hline & & GTCCTCTCAAACCCGCTA \\
\hline Helper sequence(s) & CCACATGCTCCACCGC & CAGTACCAGTGTGGCGGATC \\
\hline Positive control & Caminibacter mediatlanticus & Sulfurimonas autotrophica \\
\hline 1-mismatch & $\begin{array}{l}\text { Sulfurimonas denitrificans } \\
\text { Sulfurovum lithotrophicum }\end{array}$ & Thiomicrospira halophila \\
\hline Other controls & EPSI549/914) & \\
\hline Formamide (\%) & $30-40$ & 35 \\
\hline Predicted coverage and notes & $\begin{array}{l}\text { Zero coverage for genera Nitratifractor and Nitratiruptor, } \\
\text { otherwise excellent for Nautiliales. Competitor is for } \\
\text { Campylobacterales which are 1-mismatch from probe. }\end{array}$ & $\begin{array}{l}\text { High coverage for taxonomically } \\
\text { diverse Sulfurimonas sequences }\end{array}$ \\
\hline
\end{tabular}

Sequences presented in $5^{\prime}=>3$ ' direction 
Table A.3: Correlations between initial chemical parameters and final Sulfurimonas OTU composition after incubation for $\approx 24 \mathrm{~h}$ at in situ temperature and pressure. $\mathrm{R}^{2}$ and $\mathrm{p}$ values were determined using the script "compare_categories.py" from the QIIME pipeline (Caporaso et al., 2010). Sulfurimonas OTUs from $50{ }^{\circ} \mathrm{C}$ incubations were excluded from this analysis.

\begin{tabular}{|c|c|c|}
\hline Parameter & $\mathrm{R}^{2}$ & $\mathrm{p}$ value \\
\hline Initial $\mathrm{pO}_{2}$ & 0.52 & 0.001 \\
\hline Initial cell density & 0.18 & 0.076 \\
\hline Initial $\left[\mathrm{NO}_{3}{ }^{-}\right]$ & 0.18 & 0.076 \\
\hline Time from bottom & 0.15 & 0.101 \\
\hline Initial $\left[\mathrm{H}_{2}\right]$ & 0.1 & 0.21 \\
\hline Initial $\left[\mathrm{H}_{2} \mathrm{~S}\right]$ & 0.085 & 0.27 \\
\hline Initial $\left[\mathrm{CH}_{4}\right]$ & 0.095 & 0.028 \\
\hline Initial $\mathrm{pH}$ & 0.026 & 0.78 \\
\hline Initial $\left[\mathrm{NH}_{3}\right]$ & 0.02 & 0.88 \\
\hline
\end{tabular}


Table A.4: Correlations between abundance and $\mathrm{pO}_{2}$ for the 24 most abundant Sulfurimonas OTUs ( $\geq 2 \%$ of total) after incubation for $\approx 24 \mathrm{~h}$ at in situ temperature and pressure. Pearson correlation coefficients and p-values were determined using the script "observation_metadata_correlation.py" from the QIIME pipeline (Caporaso et al., 2010). Sulfurimonas OTUs from $50^{\circ} \mathrm{C}$ incubations were excluded from this analysis. ${ }^{*}=$ p-values corrected for multiple hypothesis testing using the Benjamini-Hochberg FDR procedure; ${ }^{* *}=$ p-values corrected for multiple hypothesis testing using the Bonferroni procedure.

\begin{tabular}{|c|c|c|c|c|}
\hline OTU ID & Pearson correlation & $p$-value & $\mathrm{p}$-value $\left(\mathrm{fdr} \mathrm{r}^{*}\right)$ & p-value (bon $\left.{ }^{* *}\right)$ \\
\hline denovo14 & 0.94 & $1.12 \times 10^{-9}$ & $2.7 \times 10^{-8}$ & $2.7 \times 10^{-8}$ \\
\hline denovo0 & 0.85 & $1.1 \times 10^{-5}$ & 0.00014 & 0.00027 \\
\hline denovo16 & -0.84 & $2.4 \times 10^{-5}$ & 0.00019 & 0.00057 \\
\hline denovo 27 & -0.78 & 0.00028 & 0.0015 & 0.00669 \\
\hline denovo5 & -0.78 & 0.00031 & 0.0015 & 0.00748 \\
\hline denovo29 & -0.76 & 0.00058 & 0.0023 & 0.014 \\
\hline denovo34 & 0.75 & 0.00076 & 0.0026 & 0.018 \\
\hline denovo31 & -0.72 & 0.0018 & 0.0055 & 0.044 \\
\hline denovo7 & 0.7 & 0.0024 & 0.0064 & 0.058 \\
\hline denovo9 & -0.7 & 0.0029 & 0.007 & 0.07 \\
\hline denovo45 & -0.67 & 0.0054 & 0.011 & 0.13 \\
\hline denovo32 & 0.66 & 0.0055 & 0.011 & 0.13 \\
\hline denovo8 & -0.66 & 0.0058 & 0.011 & 0.14 \\
\hline denovo39 & 0.66 & 0.0063 & 0.011 & 0.15 \\
\hline denovo36 & -0.64 & 0.0092 & 0.015 & 0.22 \\
\hline denovo11 & -0.63 & 0.011 & 0.016 & 0.26 \\
\hline denovo28 & -0.51 & 0.053 & 0.074 & 1 \\
\hline denovo26 & 0.49 & 0.064 & 0.085 & 1 \\
\hline denovo63 & 0.46 & 0.09 & 0.11 & 1 \\
\hline denovo2 & -0.44 & 0.1 & 0.12 & 1 \\
\hline denovo3 & 0.42 & 0.12 & 0.14 & 1 \\
\hline denovo4 & 0.42 & 0.13 & 0.14 & 1 \\
\hline denovo20 & 0.22 & 0.43 & 0.45 & 1 \\
\hline denovo21 & -0.19 & 0.5 & 0.5 & 1 \\
\hline
\end{tabular}




\section{Appendix B}

\section{Supplemental Material for Chapter 4}

The following is a plaintext table representing the metabolic model of Sulfurimonas denitrificans described in Chapter 4. Semicolons separate cells and line breaks are defined explicitly by " $\backslash n "$. Gene associations for metabolic reactions are reported where genomic evidence is available and represented as either gi \#'s or Suden_\#\#\#\# protein IDs.

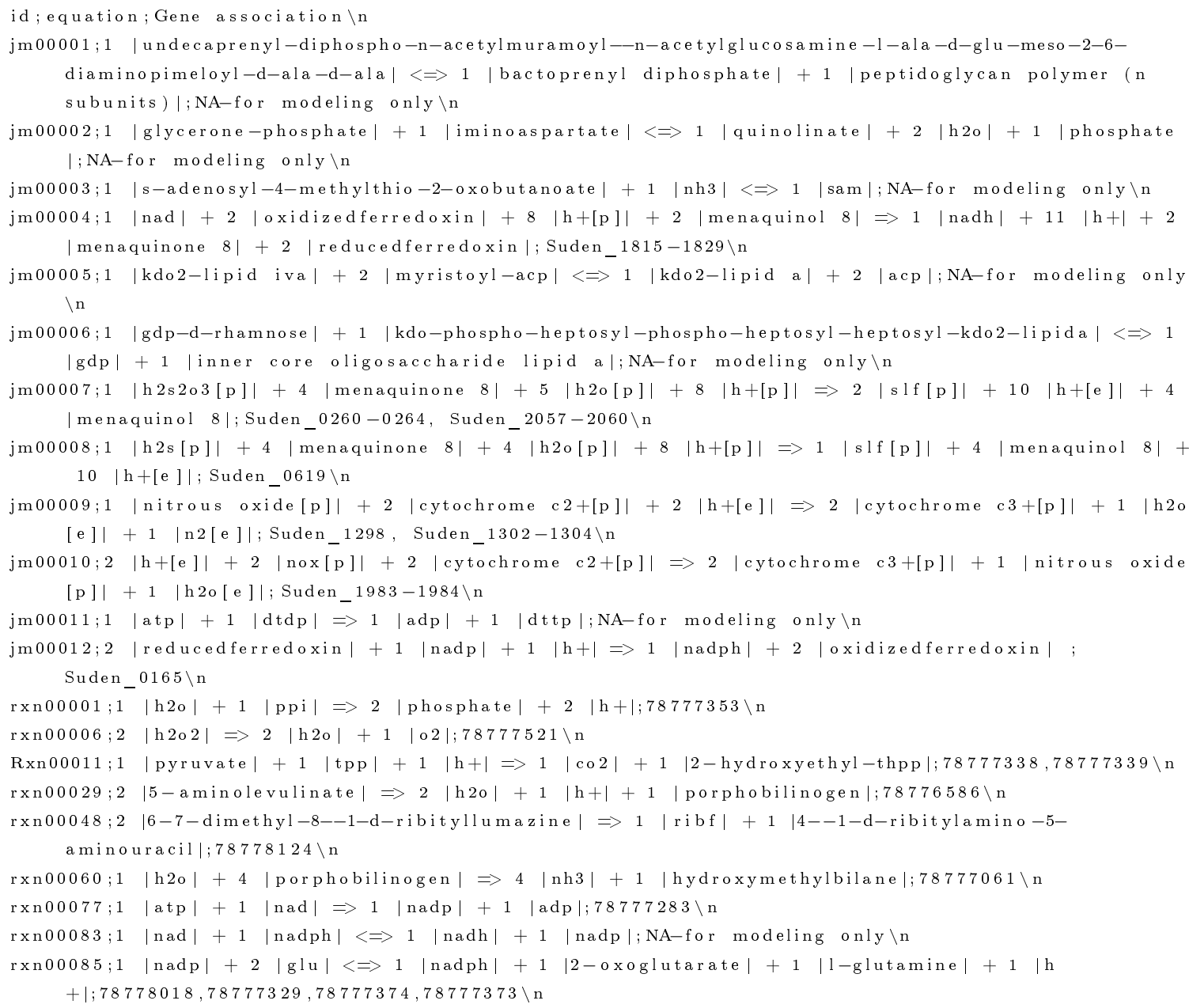




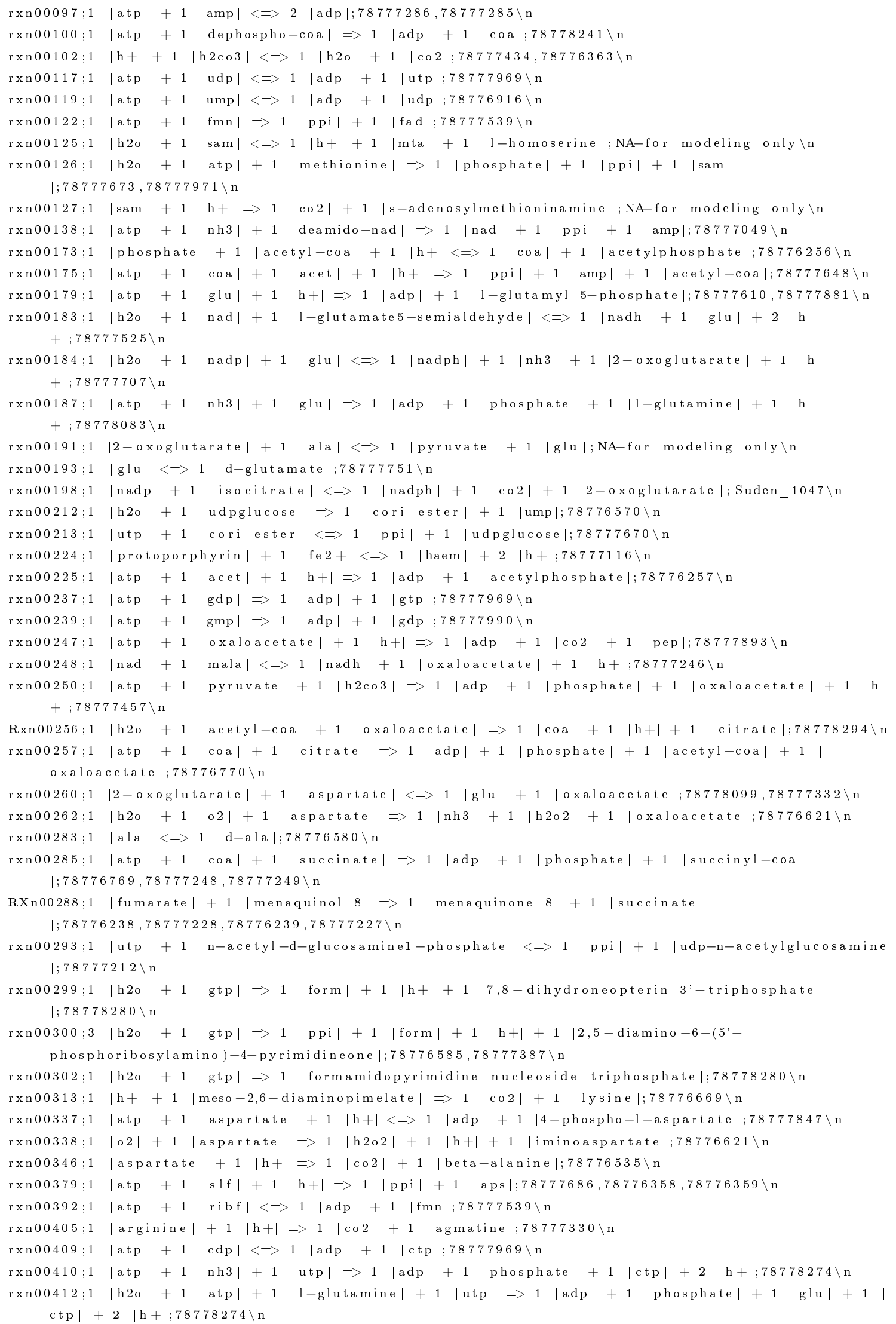


rxn00414;1 |h2o $|+2|$ atp $|+1| 1-$ glutamine $|+1|$ h2co3 $|\Rightarrow 2|$ adp $|+1|$ phosphate $|+1| g l u \mid+1$

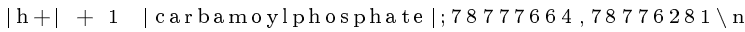

rxn00416;1 |h2o| $+1 \mid$ atp $|+1|$ aspartate $|+1| 1-$ glutamine $|\Rightarrow 1| p p i|+1|$ amp $|+1| g l u|+1| 1$ -asparagine |; $78776364,78776387,78776401 \backslash \mathrm{n}$

rxn00420;1 |h2o| + 1 |phosphoserine $\Rightarrow 1$ |phosphate| + 1 |serine | 1 |h+|;78777419,78777862\n

$\operatorname{rxn00423;1} \mid$ acetyl-coa $|+1|$ serine $\Rightarrow 1 \mid$ coa $\mid+1$ |o-acetyl-1-serine|;78777331\n

$\operatorname{rxn00438;1} \mid$ atp $|+1| \operatorname{tmp}|\Leftrightarrow 1| \operatorname{adp}|+1| \operatorname{tpp} \mid ; 78777229 \backslash \mathrm{n}$

rxn00459;1 $\mid 2$-phospho-d-glycerate $|\Leftrightarrow 1|$ h2o $|+1|$ pep $\mid ; 78778195 \backslash \mathrm{n}$

rxn00461; $\mid$ udp-n-acetylglucosamine $|+1|$ pep $|\Leftrightarrow 1|$ phosphate $|+1| \mathrm{h}+|+1|$ udp-n-

acetylglucosamine enolpyruvate $\mid ; 78776755 \backslash \mathrm{n}$

rxn00474;1 |serine $+1 \mid$ indol| $\Rightarrow 1 \mid$ h2o $\mid+1$ |tryptophan|;78777317,78776941,78777878\n

rxn00493;1 |2-oxoglutarate $|+1| 1-$ phenylalanine $|\Leftrightarrow 1|$ glu $\mid+1$ |phenylpyruvate|;78776672 $\mid$ n

rxn00503;2 |h2o $|+1|$ nad $|+1| 1-$ pyrroline-5-carboxylate $|\Longleftrightarrow 1|$ nadh $|+1|$ glu $|+1| h$

$+\mathrm{I} ; 78777525 \backslash \mathrm{n}$

rxn00527;1 |2-oxoglutarate $|+1|$ tyrosine $|\Leftrightarrow 1|$ glu| + 1 |p-hydroxyphenylpyruvate|;78776672 $\backslash \mathrm{n}$

rxn00545;1 |atp $\mid+1$ |neuberg ester $|\Rightarrow 1|$ adp $|+1|$ d-fructose1-6-bisphosphate|;78776748 $\backslash n$

rxn00549;1 |h2o| + 1 |d-fructose1-6-bisphosphate $|\Rightarrow 1|$ phosphate $|+1|$ h+| $+1 \mid$ neuberg ester $1 ; 78777261 \backslash \mathrm{n}$

rxn00555;1 |l-glutamine $\mid+1$ |neuberg ester $|\Leftrightarrow 1|$ glu| $+1 \mid$ d-glucosamine phosphate|;78777680 $\backslash$ n rxn00558;1 |robison ester $\Leftrightarrow 1$ |neuberg ester|;78777669 $\Rightarrow \mathrm{n}$

rxn00559;1 |d-mannose6-phosphate $\mid \Leftrightarrow 1$ |neuberg ester $\mid$;NA-for modeling only $\backslash n$

Rxn00567;2 |h+[e]| $+1 \mid$ nitrite [p] $|+1|$ cytochrome c $2+[p]|\Rightarrow 1|$ h2o[e]| $+1|c y t o c h r o m e c 3+[p]|$ $+1|\operatorname{nox}[\mathrm{p}]| ; 78778179 \backslash \mathrm{n}$

Rxn00611;1 |nadh $|+1| \mathrm{h}+|+1|$ glycerone-phosphate $|\Rightarrow 1|$ nad $|+1|$ glyc-3-p|;78777528 $>\mathrm{n}$

Rxn00612;1 |nadph $|+1| \mathrm{h}+|+1|$ glycerone-phosphate $|\Rightarrow 1|$ nadp $|+1|$ glyc-3-p|;78777528 $\backslash \mathrm{n}$

$\operatorname{rxn00615;1}|\operatorname{atp}|+1|\operatorname{glyc}| \Leftrightarrow 1|\operatorname{adp}|+1|\operatorname{glyc}-3-\mathrm{p}| ;$ NA-for modeling only $\backslash \mathrm{n}$

rxn00640;1 |gdp| $+1 \mid \mathrm{d}-$ mannose1-phosphate $|\Longleftrightarrow 1|$ phosphate $|+1| \mathrm{h}+|+1|$ gdp-mannose $\mid ; 78777928 \backslash$

$\mathrm{n}$

rxn00641;1 |gtp $|+1| \mathrm{d}-$ mannose1-phosphate $|\Leftrightarrow 1| \mathrm{ppi}|+1| \mathrm{gdp}-\mathrm{mannose} \mid ; 78776376 \backslash \mathrm{n}$

rxn00642;1 |gdp-mannose $\Rightarrow 1 \mid$ h2o $\Rightarrow+1 \mid$ gdp-4-dehydro-d-rhamnose|;78777900 \n

rxn00649;1 |h2s $|+1|$ o-acetyl-1-serine $\Rightarrow 1 \mid$ acet $|+1| \mathrm{h}+|+1| 1-$ cysteine|;78776355,78776634 $\backslash \mathrm{n}$

$\operatorname{rxn00686;1}|\operatorname{nadp}|+1|\mathrm{thf}| \Leftrightarrow 1 \mid$ nadph $|+1| \mathrm{h}+|+1|$ dihydrofolate|;78777844 $\mid \mathrm{n}$

$\operatorname{rxn00688;1}|\mathrm{h} 2 \mathrm{o}|+1|\operatorname{nadp}|+1 \mid 10-$ formyl-thf $|\Leftrightarrow 1| \operatorname{nadph}|+1| \operatorname{co} 2|+1| \mathrm{h}+|+1| \mathrm{thf} \mid ; \mathrm{NA}-\mathrm{for}$ modeling only $\backslash \mathrm{n}$

$\operatorname{rxn00691;1}|\mathrm{h} 2 \mathrm{o}|+1 \mid 10-$ formyl-thf $\Rightarrow 1 \mid$ form $|+1| \mathrm{h}+|+1| \mathrm{thf} \mid ; 78777296 \backslash \mathrm{n}$

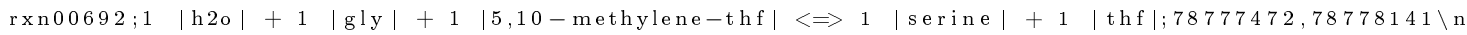

rxn00693;1 |homocysteine $|+1| 5$-methyltetrahydrofolate $|\Longleftrightarrow 1|$ methionine $|+1|$ thf $\mid ; 78778225 \backslash \mathrm{n}$

rxn00704;1 |cori ester| $\Rightarrow 1$ |robison ester|;78776822\n

rxn00710;1 |h+l + 1 orotidylic acid $\Rightarrow 1|\operatorname{co} 2|+1|u m p| ; 78777644 \backslash \mathrm{n}$

rxn00726;1 |nh3| + 1 |chorismate $\Rightarrow 1 \mid$ h2o $|+1|$ pyruvate $|+1| h+|+1|$ vitamin 11 I; $78777475,78776767 \backslash \mathrm{n}$

rxn00727;1 |l-glutamine $\mid+1$ |chorismate $\mid \Rightarrow 1$ |pyruvate $|+1|$ glu| $+1|\mathrm{~h}+|+1 \mid$ vitamin 11 |; $78777475,78776767 \backslash \mathrm{n}$

rxn00737;1 |threonine $\Rightarrow 1|\mathrm{nh} 3|+1 \mid 2$-oxobutyrate|;78777341 $\backslash \mathrm{n}$

rxn00740;1 |h2o| + 1 |o-succinyl-1-homoserine $|\Rightarrow 1| n h 3|+1|$ succinate $|+1| h+|+1| 2-$ oxobutyrate|; $78776660,78776964 \backslash \mathrm{n}$

rxn00747;1 |glyceraldehyde3-phosphate $\Leftrightarrow$ | $\Leftrightarrow$ glycerone-phosphate|;78777946 $\backslash \mathrm{n}$

rxn00770;1 |atp| + 1 |ribose-5-phosphate $\Leftrightarrow 1|\operatorname{amp}|+1$ |prpp|;78777833\n

rxn00777;1 |ribose-5-phosphate| $\Leftrightarrow 1$ |d-ribulose5-phosphate|;78777314 $\backslash \mathrm{n}$

rxn00781; $\mid$ nad $|+1|$ phosphate $|+1|$ glyceraldehyde 3 -phosphate $|<\Rightarrow 1|$ nadh $|+1| 1,3-$ bisphosphod-glyceratel; $78777948 \backslash \mathrm{n}$

rxn00785;1 |neuberg ester $\mid+1$ |glyceraldehyde3-phosphate $|\Leftrightarrow 1| d-x y l u l o s e 5-p h o s p h a t e|+1| d-$ erythrose 4 -phosphatel; $78776382,78776539,78776381 \backslash \mathrm{n}$

rxn00786;1 |d-fructose1-6-bisphosphate $\mid \Leftrightarrow 1$ |glycerone-phosphate | 1 |glyceraldehyde3-phosphate |; $78776709 \backslash \mathrm{n}$

Rxn00789;1 |atp $|+1| \operatorname{prpp}|\Rightarrow 1|$ ppi $|+1|$ phosphoribosyl-atp|;78776275,78777087 $>\mathrm{n}$

Rxn00790;1 |h2o| + $1 \mid 1$-glutamine $|+1| \operatorname{prpp}|\Rightarrow 1|$ ppi $|+1|$ glu| 1 |5-phosphoribosylamine I; $78778065 \backslash \mathrm{n}$

Rxn00791;1 |vitamin $11|+1| \operatorname{prpp}|\Rightarrow 1|$ ppi| + $1 \mid$ n-5-phosphoribosyl-anthranilate $\mid ; 78777349 \backslash$ n

rxn00799;1 |mala $|\Leftrightarrow 1|$ h2o $|+1|$ fumarate|; $78777247 \backslash \mathrm{n}$

rxn00800;1 |adenylosuccinate $|\Leftrightarrow 1| \operatorname{amp}|+1 \quad|$ fumarate|;78778289 $\backslash \mathrm{n}$ 
rxn00802;1 |1-argininosuccinate $|\Leftrightarrow 1|$ arginine $|+1 \quad|$ fumarate $\mid ; 78777064 \backslash \mathrm{n}$

rxn00806;1 |2-oxoglutarate $|+1| 1-1$ eucine $|\Leftrightarrow 1| g l u|+1| 4 \operatorname{mop} \mid ; 78778071 \backslash \mathrm{n}$

$\operatorname{rxn00832;1}|\mathrm{h} 2 \mathrm{o}|+1|\mathrm{imp}| \Leftrightarrow 1$ |faicar $\mid ; 78776749 \backslash \mathrm{n}$

$\operatorname{rxn00834;1}|\mathrm{h} 2 \mathrm{o}|+1|\operatorname{nad}|+1|\mathrm{imp}| \Leftrightarrow 1|\operatorname{nadh}|+1|\mathrm{~h}+|+1|\operatorname{xmp}| ; 78776969 \backslash \mathrm{n}$

rxn00838;1 |gtp| $+1 \mid$ aspartate $|+1|$ imp $|\Rightarrow 1|$ phosphate $|+1| \operatorname{gdp}|+2| \mathrm{h}+|+1|$

adenylosuccinate |; $78776276 \backslash \mathrm{n}$

$\operatorname{rxn00839;1}|\operatorname{atp}|+1|\operatorname{dadp}| \Rightarrow 1|\operatorname{adp}|+1|\operatorname{datp}| ; 78777969 \backslash \mathrm{n}$

$\operatorname{rxn00851;1} \mid$ atp $|+2| \mathrm{d}-\mathrm{ala}|\Rightarrow 1| \operatorname{adp}|+1|$ phosphate $|+1| \mathrm{h}+|+1 \quad|$ ala-ala|;78777406\n

rxn00853;1 |h2o| $+2|\mathrm{~h}+|+1 \mid \mathrm{n}-$ carbamoylputrescine $|\Rightarrow 1| \operatorname{co} 2|+1| \mathrm{nh} 3|+1| \mathrm{putr}$

।; $78776215,78776801,78778038 \backslash \mathrm{n}$

rxn00863; $\mid$ h2o $|+1|$ nad $|+1| 1$-histidinal $|\Rightarrow 1|$ nadh $|+2| h+|+1| 1-$ histidine $\mid ; 78776697 \backslash n$

rxn00898;1 $\mid 2,3$-dihydroxy-isovalerate $\Rightarrow 1 \mid$ h2o $|+1| 3 \operatorname{mob} \mid ; 78776266 \backslash \mathrm{n}$

Rxn00902;1 |h2o|+1|acetyl-coa $|+1| 3 \mathrm{mob}|\Rightarrow 1|$ coa $|+1| \mathrm{h}+|+1| 2-$ isopropylmalate $\mid$ $; 78778084 \backslash \mathrm{n}$

rxn00903;1 |2-oxoglutarate $|+1| 1-$ valine $|\Leftrightarrow 1 \quad|$ glu $|+1 \quad| 3 \operatorname{mob} \mid ; 78778071 \backslash \mathrm{n}$

rxn00907;1 $\mid$ nadp $|+1| 5,10-$ methylene-thf $|\Leftrightarrow 1|$ nadph $|+1| 5-10-$ methenyltetrahydrofolate |; $78777311 \backslash \mathrm{n}$

rxn00910;1 |nadp $|+1| 5-$ methyltetrahydrofolate $|<\Rightarrow 1|$ nadph $|+1|$ h+| $+1 \mid 5,10-$ methylene-thf

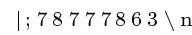

$\operatorname{rxn00912;1}|\mathrm{h} 2 \mathrm{o}|+1|3 \mathrm{mob}|+1 \mid 5,10$ - methylene-thf $|\Leftrightarrow 1| \mathrm{thf}|+1| 2-$ dehydropantoate $\mid ; 78777876 \backslash$ $\mathrm{n}$

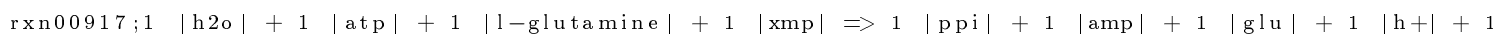
$|\mathrm{gmp}| ; 78776623 \backslash \mathrm{n}$

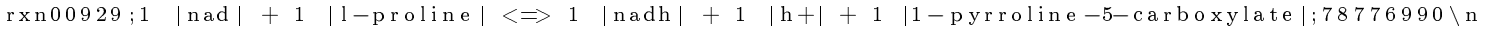

rxn00952;1 |h2s $|+1|$ o-acetyl-1-homoserine $|\Rightarrow 1|$ acet $|+1| \mathrm{h}+\mid+1$ |homocysteine I; $78776660,78776964 \backslash \mathrm{n}$

rxn00973;1 |citrate $\Leftrightarrow 1 \quad$ isocitrate|;78777238\n

rxn01000;1 |h+l + 1 |prephenate $\Rightarrow 1|\mathrm{~h} 2 \mathrm{o}|+1|\cos |+1 \mid$ phenylpyruvate|;78776671 $\backslash \mathrm{n}$

rxn01018;1 |aspartate | + 1 |carbamoylphosphate $\mid \Rightarrow 1$ |phosphate $|+2| h+|+1| n-c a r b a m o y l-1-$ aspartate|; $78776662 \backslash \mathrm{n}$

rxn01019;1 |ornithine | 1 |carbamoylphosphate $|\Rightarrow 1|$ phosphate $|+2|$ h+| $+1 \mid$ citrulline I; $78776587 \backslash \mathrm{n}$

rxn01029;1 |h2o| + 1 |agmatine $\Rightarrow 1|\mathrm{nh} 3|+1 \mid \mathrm{n}$-carbamoylputrescine|;78776579 $>\mathrm{n}$

rxn01069;1 |h2o| + 1 |o-phospho-l-homoserine $|\Rightarrow 1|$ phosphate $|+1|$ h+| $+1 \mid$ threonine|;78776988 $\backslash$ n

rxn01100;1 |atp $|+1| \mathrm{h}+|+1| 3$-phosphoglycerate $|\Leftrightarrow 1|$ adp $|+1| 1,3$-bisphospho-d-glycerate |; $78777947 \backslash \mathrm{n}$

rxn01101;1 |nad $|+1| 3$-phosphoglycerate $|\Leftrightarrow 1|$ nadh $|+1| h+|+1| 3$ - phosphonooxypyruvate 1; $78777073 \backslash \mathrm{n}$

rxn01106;1 $\mid 2$-phospho-d-glycerate $|\Leftrightarrow 1| 3-$ phosphoglycerate $\mid ; 78777811 \backslash \mathrm{n}$

rxn01116;1 |d-ribulose 5-phosphate $|\Leftrightarrow 1 \quad| d-x y l u l o s e 5-p h o s p h a t e \mid ; 78777802 \backslash \mathrm{n}$

rxn01117;1 |d-arabinose5-phosphate $|\Leftrightarrow 1 \quad| d-r i b u l o s e 5-p h o s p h a t e \mid ; 78776414 \backslash n$

rxn01200;1 |glyceraldehyde3-phosphate | 1 |sedoheptulose7-phosphate | $<1 \mid$ ribose-5-phosphate | 1 |d-xylulose 5 -phosphate|;78776382,78776539,78776381\n

$\operatorname{rxn01207;1}|\operatorname{nad}|+1 \mid$ coa $|+1| 4 \operatorname{mop}|\Leftrightarrow 1|$ nadh $|+1| \operatorname{co} 2|+1|$ isovaleryl-coa $\mid$; NA-for modeling on $1 \mathrm{y} \backslash \mathrm{n}$

Rxn01208;1 $|\mathrm{h}+|+1 \mid 2$-isopropyl-3-oxosuccinate $|\Rightarrow 1| \operatorname{co} 2|+1| 4$ mop $\mid ;$ NA-for modeling only $\backslash \mathrm{n}$

rxn01211;1 $|\mathrm{h} 2 \mathrm{o}|+1 \mid 5-10-$ methenyltetrahydrofolate $|<\Leftrightarrow 1| \mathrm{h}+|+1| 10-$ formyl-thf $\mid ; 78777311 \backslash \mathrm{n}$

rxn01255; $\mid 5-0--1-$ carboxyvinyl-3-phosphoshikimate $\mid \Rightarrow 1$ phosphate $|+1| h+|+1|$ chorismate I; $78778217 \backslash \mathrm{n}$

rxn01256;1 |chorismate | $\Rightarrow 1$ |prephenate|;78776671 \n

rxn01257;1 |l-glutamine $\mid+1$ |chorismate $|\Leftrightarrow 1|$ glu $|+1|$ adc|;78776663,78776767\n

rxn01268;1 |nad $|+1|$ prephenate $|\Rightarrow 1|$ nadh $|+1|$ co $2 \mid+1$ |p-hydroxyphenylpyruvate|;78776858\n

rxn01269;1 |nadp $|+1|$ prephenate $|\Rightarrow 1|$ nadph $|+1|$ co2 $\mid+1$ |p-hydroxyphenylpyruvate|;78776858 $\backslash$ n

rxn01300;1 |atp $|+1| 1$-homoserine $|\Leftrightarrow 1|$ adp $\mid+1$ |o-phospho-l-homoserine|;78776873 \n

rxn01301;1 |nad $|+1| 1$-homoserine $|\Leftrightarrow 1|$ nadh $|+1|$ h $+|+1| 1$-aspartate4-semialdehyde I; $78778098 \backslash \mathrm{n}$

rxn01303;1 |acetyl-coa| + 1 |1-homoserine $\Rightarrow 1 \mid$ coa | + 1 |o-acetyl-1-homoserine|;78776963 \n

rxn01329;1 |d-mannose6-phosphate $|\Leftrightarrow 1 \quad| d$-mannose1-phosphate|;78776822 \n

rxn01331; $\mid$ h2o $|+1|$ nad $|+1|$ d-erythrose4-phosphate $|\Rightarrow 1|$ nadh $|+2| h+|+1| 4-$

phosphoerythronate|; $78777948 \backslash \mathrm{n}$ 


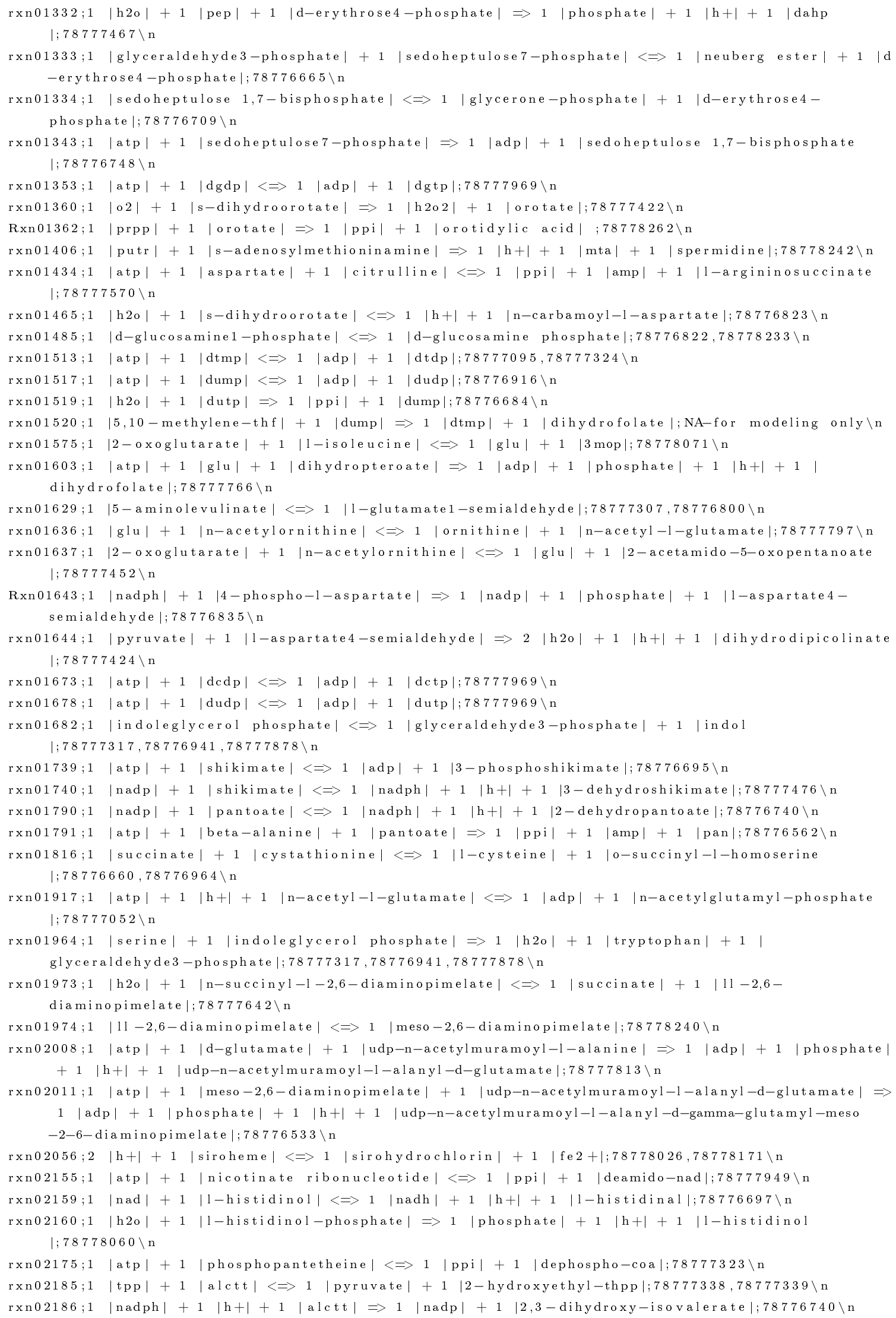


rxn02187;1 |alctt $\Leftrightarrow 1 \mid 2-$ oxo-3-hydroxyisovalerate|; $78776740 \backslash \mathrm{n}$

rxn02200;1 |abee| $+1 \mid 6$-hydroxymethyl dihydropterin $|\Leftrightarrow 1|$ h2o| +1 |dihydropteroate|;78777844 $\Rightarrow$

rxn02201;1 |abee| + 1 |7,8-dihydropterin pyrophosphate $\Rightarrow 1 \mid$ ppi $\mid+1$ |dihydropteroate|;78777844 $\backslash$

$\mathrm{n}$

rxn02212;1 |dahp| $\Rightarrow 1$ |phosphate $|+1| \mathrm{h}+|+1| 5$-dehydroquinate|;78777218\n

rxn02213;1 $\mid 5$-dehydroquinate $|\Rightarrow 1|$ h2o $|+1| 3$-dehydroshikimate|;78776886 \n

rxn02264;1 |hydroxymethylbilane $\Leftrightarrow 1 \mid$ h2o $\Leftrightarrow 1$ |uroporphyrinogeniii|;78776624,78777426\n

$\operatorname{rxn02269;1}|\operatorname{nad}|+1 \mid$ coa $|+1| 3 \mathrm{mop}|\Leftrightarrow 1|$ nadh $|+1| \operatorname{co} 2|+1| 2-$ methylbutyryl-coa $\mid$; NA-for modeling only $\backslash \mathrm{n}$

rxn02277;1 |atp $|+1| \operatorname{co} 2|+1| 7-8$-diaminononanoate $|\Rightarrow 1| \operatorname{adp}|+1|$ phosphate $|+3|$ h+ $|+1|$ dethiobiotin|; $78776661 \backslash \mathrm{n}$

rxn02285;1 |nadp $|+1|$ udp-murnac $|\Leftrightarrow 1|$ nadph $|+1| \mathrm{h}+|+1|$ udp-n-acetylglucosamine enolpyruvate $\mid ; 78778200 \backslash \mathrm{n}$

rxn02286;1 |atp $|+1|$ ala $|+1|$ udp-murnac $|\Rightarrow 1|$ adp $|+1|$ phosphate $|+1| h+|+1| u d p-n-$ acetylmuramoyl-1-alanine |; $78776956 \backslash \mathrm{n}$

rxn02287;2 |sam $|+1|$ uroporphyrinogeniii $|\Leftrightarrow 2| s-a d e n o s y l h o m o c y s t e i n e|+2| h+|+1|$ precorrin $2 \mid ; 78778183,78777645 \backslash \mathrm{n}$

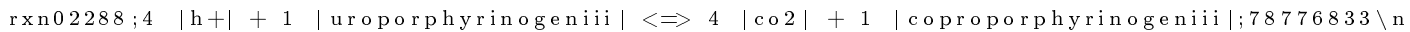

rxn02297; $\mid$ ala $|+1| \mathrm{h}+|+1|$ pimeloyl-coa $|\Rightarrow 1|$ coa $|+1|$ co $|+1| 8$-amino-7-oxononanoate I; $78778088 \backslash \mathrm{n}$

rxn02302;1 |1-cysteine $|+1|$ o-acetyl-1-homoserine $|\Leftrightarrow 1|$ acet $\mid+1$ |cystathionine I; $78776660,78776964 \backslash \mathrm{n}$

rxn02303;1 |o2| + $2|\mathrm{~h}+|+1 \mid$ coproporphyrinogeniii $|\Rightarrow 2| h 20|+2| \cos |+1|$ protoporphyrinogenix |; $78777851 \backslash \mathrm{n}$

rxn02304;3 |o2| + 2 |protoporphyrinogenix $\Rightarrow 6 \mid$ h2o $\mid+2$ |protoporphyrin $\mid$;NA-for modeling only $\backslash$ n rxn02305;1 |h+l + 1 |4-methyl-5--2-phosphoethyl-thiazole | + 1 |4-amino-2-methyl-5diphosphomethylpyrimidine $|\Leftrightarrow 1|$ ppi $\mid+1$ |tmp|;78777371,78777436,78778249\n rxn02312;1 |sam $|+1| 8$-amino-7-oxononanoate $|\Leftrightarrow 1| 7-8$-diaminononanoate $|+1| s-a d e n o s y l-4-$ methylthio-2-oxobutanoatel; $78777488 \backslash \mathrm{n}$

rxn02320;1 |2-oxoglutarate $|+1| 1$-histidinol-phosphate $|\Leftrightarrow 1|$ glu| +1 |imidazole acetolphosphate $\mid ; 78776672 \backslash \mathrm{n}$

Rxn02331;1 |h2o| + 1 |pep $|+1|$ d-arabinose 5-phosphate $|\Rightarrow 1|$ phosphate $|+1| h+|+1| 3-$ deoxy-dmanno-octulosonate 8 - phosphate|; $78776602 \backslash \mathrm{n}$

rxn02341;1 |h+l + $1 \mid(\mathrm{r})-4$ - phosphopantothenoyl-1-cysteine $|\Rightarrow 1|$ co $2|+1|$ phosphopantetheine I;78777196\n

Rxn02373;1 |nadph| + 1 |1-glutamyl 5-phosphate $|\Rightarrow 1|$ nadp $|+1|$ phosphate $|+1| 1-g l u t a m a t e 5-$ semialdehyde $\mid ; 78776991 \backslash \mathrm{n}$

Rxn02402;2 |h+| $+1 \mid$ prpp $|+1|$ quinolinate $|\Rightarrow 1| \operatorname{co} 2|+1|$ ppi $\mid+1$ |nicotinate ribonucleotide ।; $78776867,78777737 \backslash \mathrm{n}$

rxn02404;1 |h2o| + $1 \mid 3$-deoxy-d-manno-octulosonate8-phosphate $|\Rightarrow 1|$ phosphate $|+1| \mathrm{h}+|+1| \mathrm{kdo}$ I; $78777277 \backslash \mathrm{n}$

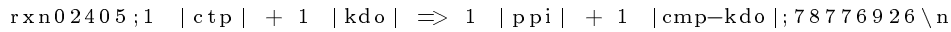

rxn02438;1 |nad $|+1|$ gdp-d-rhamnose $|\Leftrightarrow 1|$ nadh $|+1|$ h $+|+1|$ gdp-4-dehydro-d-rhamnose|;NA-for modeling only $\backslash \mathrm{n}$

Rxn02465;1 |nadph | + 1 |n-acetylglutamyl-phosphate $|\Rightarrow 1|$ nadp $|+1|$ phosphate $|+1| 2-$ acetamido $-5-$ oxopentanoatel; $78777730 \backslash \mathrm{n}$

rxn02473;1 |d-erythro-imidazol-glycerol-phosphate $|\Rightarrow 1|$ h2o $|+1|$ imidazole acetol-phosphate I; $78777278 \backslash \mathrm{n}$

rxn02474;1 |nadp $|+1| 5-$ amino-6--5-phosphoribitylaminouracil $|<\Rightarrow 1|$ nadph $|+1| \mathrm{h}+|+1| 5-$ amino $-6--5-$ phosphoribosylaminouracil|; $78776878 \backslash \mathrm{n}$

rxn02475;1 |h2o| $+1|\mathrm{~h}+|+1 \mid 2,5$-diamino-6-(5'-phosphoribosylamino $)-4-p y r i m i d i n e o n e|\Rightarrow 1|$ nh3 $\mid$ $+1 \mid 5-$ amino-6--5-phosphoribosylaminouracil|; $78776878 \backslash \mathrm{n}$

rxn02476;1 |pep| + $1 \mid 3-$ phosphoshikimate $|\Leftrightarrow 1|$ phosphate $|+1| h+|+1| 5-0--1-c a r b o x y v i n y l-3-$ phosphoshikimate|; $78777069 \backslash \mathrm{n}$

rxn02503;1 |atp $|+1| 6$-hydroxymethyl dihydropterin $|\Leftrightarrow 1|$ amp $|+1| 7,8$-dihydropterin pyrophosphate |; $78776887 \backslash \mathrm{n}$

rxn02504;1 |dihydroneopterin $\Leftrightarrow 1$ |glycolaldehyde $\mid+1$ |6-hydroxymethyl dihydropterin ।; $78776864,78776759 \backslash \mathrm{n}$

rxn02507;1 |h+| + $1 \mid 1-(2$-carboxyphenylamino $)-1$-deoxyribulose 5 -phosphate $|\Rightarrow 1|$ h2o $|+1|$ co $2 \mid+$ 1 | indoleglycerol phosphate|; $78776930 \backslash \mathrm{n}$ 


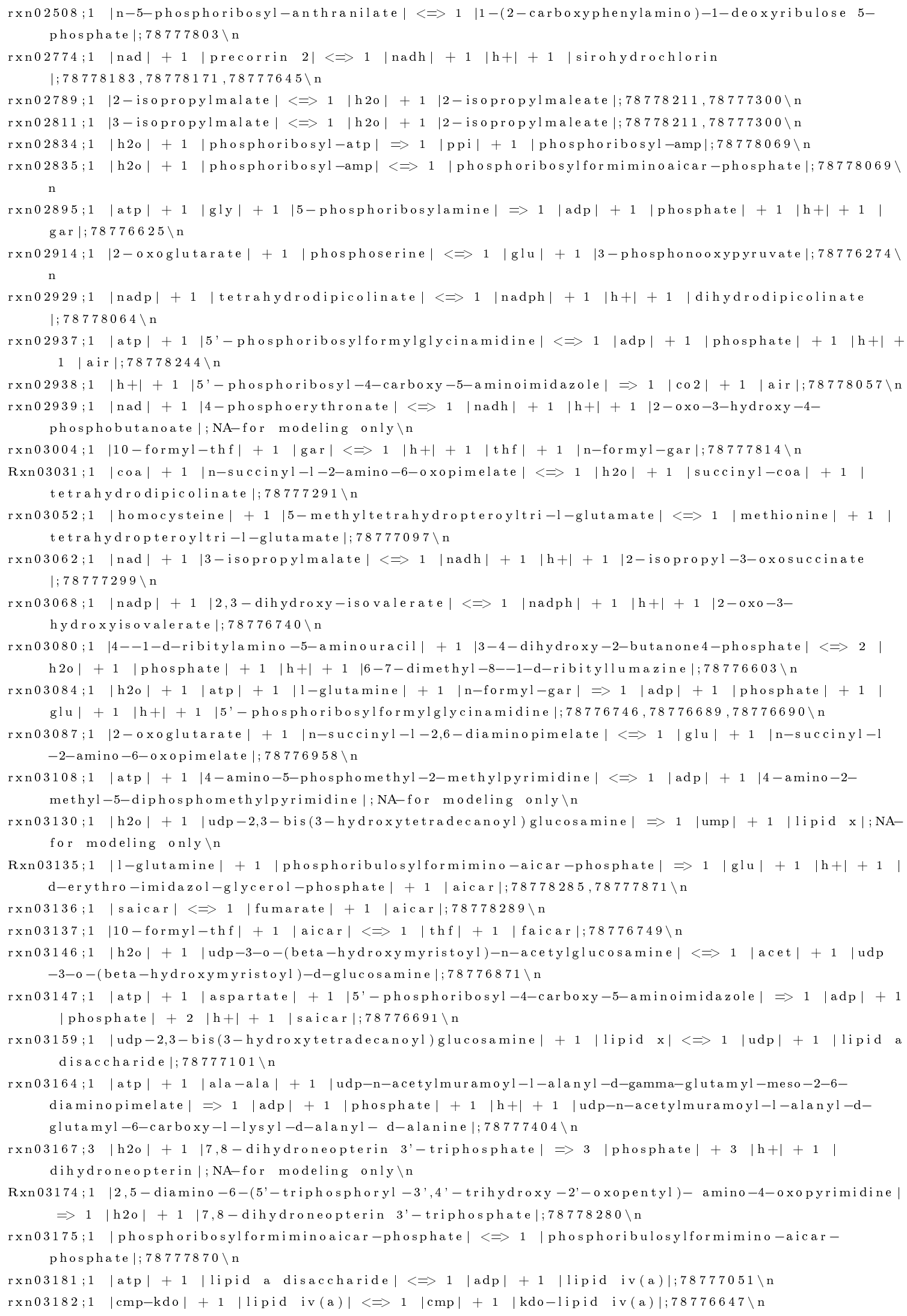


rxn03194;1 |2-oxobutyrate $|+1| 2$-hydroxyethyl-thpp $|\Leftrightarrow 1|$ tpp $|+1| 2$-aceto-2-hydroxybutanoate |; $78777338,78777339 \backslash \mathrm{n}$

rxn03408; | udp-n-acetylglucosamine | + 1 | undecaprenyl-diphospho-n-acetylmuramoyl-1-alanyl-dglutamyl-meso-2-6-diaminopimeloyl-d-alanyl-d-alanine $|\Longleftrightarrow 1| u d p \mid+1$ |undecaprenyl-diphosphon-acetylmuramoyl--n-acetylglucosamine-1-ala-d-glu-meso-2-6-diaminopimeloyl-d-ala-d-ala I; $78778001 \backslash \mathrm{n}$

rxn03419;1 |h2o| + 1 |formamidopyrimidine nucleoside triphosphate $|<\Rightarrow 1|$ form $|+1|$ h $+|+1| 2,5-$ diaminopyrimidine nucleoside triphosphate $\mid ; 78778280 \backslash \mathrm{n}$

rxn03421;1 |2,5-diaminopyrimidine nucleoside triphosphate $|\Longleftrightarrow 1| 2,5$-diamino-6-(5'-triphosphoryl -3 ', 4' - trihydroxy -2 '-oxopentyl) - amino-4-oxopyrimidine $\mid ; 78778280 \backslash \mathrm{n}$

rxn03435;1 |nadp| + $1 \mid 2,3$-dihydroxy-3-methylvalerate $|\Leftrightarrow 1|$ nadph $|+1| \mathrm{h}+|+1|(\mathrm{r})-3-\mathrm{hydroxy}$ -3 -methyl-2-oxopentanoate|; $78776740 \backslash \mathrm{n}$

rxn03436;1 |2-aceto-2-hydroxybutanoate $|\Leftrightarrow 1 \quad|(\mathrm{r})-3-$ hydroxy-3-methyl-2-oxopentanoatel;78776740 $\backslash \mathrm{n}$ rxn03437;1 $\mid 2,3$-dihydroxy-3-methylvalerate $|\Rightarrow 1|$ h2o $|+1| 3 \operatorname{mop} \mid ; 78776266 \backslash \mathrm{n}$

rxn03439;1 |cmp-kdo| $+1 \mid$ kdo-1ipid iv(a) $|\Leftrightarrow 1| \mathrm{cmp}|+1| \mathrm{kdo} 2-1 \mathrm{ipid}$ iva|;78776647\n

rxn03445;1 |2-oxoglutarate $|+1| 4-$ (phosphonooxy)-threonine $|<1|$ glu $|+1| 2-0 x o-3-h y d r o x y-4-$ phosphobutanoate|; $78776274 \backslash \mathrm{n}$

rxn03511;1 |adp-d-glycero-d-manno-heptose $\quad \Longleftrightarrow 1 \quad \mid$ adp-1-glycero-d-manno-heptose|;78776778 $\backslash \mathrm{n}$

rxn03638;1 |acetyl-coa $|+1|$ d-glucosamine1-phosphate $|\Rightarrow 1|$ coa $|+1| h+|+1| n-a c e t y l-d-$ glucosamine $1-$ phosphatel;78777212\n

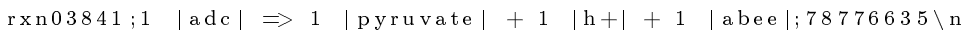

rxn03901; $\mid$ h2o $\mid+1$ |bactoprenyl diphosphate $|\Rightarrow 1|$ phosphate $|+1| h+|+1|$ undecaprenylphosphate |; $78776273 \backslash \mathrm{n}$

rxn03904;1 | undecaprenylphosphate | + 1 |udp-n-acetylmuramoyl-1-alanyl-d-glutamyl-6-carboxy-1-1ysyl $-\mathrm{d}-$ alanyl-d-alanine $|\Leftrightarrow 1| \mathrm{ump} \mid+1$ | undecaprenyl-diphospho-n-acetylmuramoyl-1-alanyl-dglutamyl-meso-2-6-diaminopimeloyl-d-alanyl-d-alanine |; $78777812 \backslash \mathrm{n}$

rxn03909;1 |pyruvate $|+1| \mathrm{h}+\mid+1$ |glyceraldehyde3-phosphate $|\Rightarrow 1| \operatorname{co} 2|+1| 1-$ deoxy-d-xylulose 5 -phosphate|; $78776676 \backslash \mathrm{n}$

rxn03916;1 |atp $|+1|$ d-glycero-d-manno-heptose1-phosphate $|\Longleftrightarrow 1|$ ppi | $+1 \mid$ adp-d-glycero-d-manno -heptose|; $78776779,78776377 \backslash \mathrm{n}$

rxn03917;1 |sedoheptulose7-phosphate | $\Rightarrow 1$ |d-glycero-d-manno-heptose7-phosphate

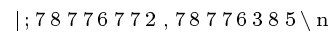

rxn03918;1 |atp $|+1|$ d-glycero-d-manno-heptose7-phosphate $|\Leftrightarrow 1|$ adp $|+1| d-$ glycero-d-manno-

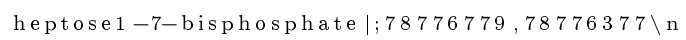

rxn03919;1 |h2o| + $1 \mid$ d-glycero-d-manno-heptose1-7-bisphosphate $|\Rightarrow 1|$ phosphate $|+1|$ h+| $+1 \mid d-$ glycero-d-manno-heptose 1 -phosphate|; $78776773 \backslash \mathrm{n}$

rxn03951;1 |nad $|+1| 4-$ (phosphonooxy)-threonine $|\Rightarrow 1|$ nadh $|+1| h+|+1| 2-a m i n o-3-o x o-4-$ phosphonooxybutyrate|; $78777093 \backslash \mathrm{n}$

rxn04070;1 |1-deoxy-d-xylulose5-phosphatel + $1 \mid 3$-amino-2-oxopropyl phosphate| $\Rightarrow 2 \mid$ hol $+1 \mid$ phosphatel $+2 \mid \mathrm{h}+\mathrm{I}+1$ |pyridoxine phosphatel;78777094 $\backslash \mathrm{n}$

rxn04704;2 |sam $\mid+1$ |coproporphyrinogeniii $\Rightarrow 2|c o 2|+2 \mid$ methionine $\mid+1$ |protoporphyrinogenix $|+2| 5^{\prime}-$ deoxyadenosine $\mid ; 78776805 \backslash \mathrm{n}$

rxn04783;1 |atp $|+1|$ form $|+1|$ gar $|\Rightarrow 1| \operatorname{adp}|+1|$ phosphate $|+1| h+|+1| n-$ formyl-gar

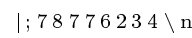

rxn05039; $\mid$ h $20|+1| 5-$ amino-6--5-phosphoribitylaminouracil $|\Rightarrow 1|$ phosphate $|+1| \mathrm{h}+|+1| 4--1-$ d-ribitylamino-5-aminouracil $\mid$; N-for modeling only $\backslash \mathrm{n}$

rxn05040;1 |d-ribulose 5-phosphate $|\Rightarrow 1|$ form $|+1| \mathrm{h}+|+1| 3-4$-dihydroxy-2-butanone4-phosphate I; $78776585,78777387 \backslash \mathrm{n}$

rxn05116;1 |h+| + $1 \mid 2$-amino-3-oxo-4-phosphonooxybutyrate $|\Rightarrow 1|$ co $2|+1| 3-$ amino-2-oxopropy 1 phosphate | NA-for modeling only $\backslash \mathrm{n}$

rxn05229;1 |atp $|+1|$ h2co3 $|+1|$ air $|\Rightarrow 1| \operatorname{adp}|+1|$ phosphate $|+1| h+|+1| 5$ - phosphoribosyl -4-carboxy-5-aminoimidazole|; $78778057 \backslash \mathrm{n}$

$\operatorname{rxn05233;1}|\operatorname{gdp}|+1|\operatorname{trdrd}| \Rightarrow 1|\mathrm{~h} 2 \mathrm{o}|+1|\operatorname{dgdp}|+1|\operatorname{trdox}| ; 78776214,78778290 \backslash \mathrm{n}$

$\operatorname{rxn05234;1}|\operatorname{gtp}|+1|\operatorname{trdrd}| \Rightarrow 1|\mathrm{~h} 2 \mathrm{o}|+1|\operatorname{dgtp}|+1|\operatorname{trdox}| ; 78777437 \backslash \mathrm{n}$

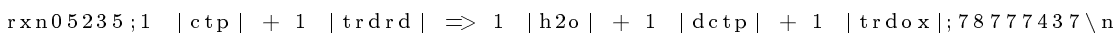

$\operatorname{rxn05236;1} \mid$ utp $|+1| \operatorname{trdrd}|\Rightarrow 1| \mathrm{h} 2 \mathrm{o}|+1| \operatorname{dutp}|+1| \operatorname{trdox} \mid ; 78777437 \backslash \mathrm{n}$

$\operatorname{rxn05256;1}|\operatorname{aps}|+1|\operatorname{trdrd}| \Rightarrow 1|\operatorname{amp}|+1|\mathrm{~h} 2 \mathrm{so} 3|+1|\operatorname{trdox}| ; 78776357 \backslash \mathrm{n}$

$\operatorname{rxn05289;1} \mid$ nadph $|+1| \mathrm{h}+|+1| \operatorname{trdox}|\Rightarrow 1| \operatorname{nadp}|+1| \operatorname{trdrd} \mid ; 78776366,78778063 \backslash \mathrm{n}$

Rxn05322;1 |nadh $|+1| \mathrm{h}+|+1|$ but-2-enoyl-[acyl-carrier protein $]|\Rightarrow 1|$ nad $|+1|$ butyryl-acp |; $78777945 \backslash \mathrm{n}$ 
Rxn05323;1 |nadh $|+1| \mathrm{h}+|+1|$ (2e)-tetradecenoyl-[acp] $\Rightarrow 1 \mid$ nad $|+1|$ myristoyl-acp|;78777945

Rxn05324;1 |nadh $|+1| \mathrm{h}+|+1|(2 \mathrm{e})$-dodecenoyl-[acp $]|\Rightarrow 1|$ nad $\mid+1$ |dodecanoyl-acp|;78777945 $\mid \mathrm{n}$ Rxn05325;1 |nadh $|+1| \mathrm{h}+|+1|(2 \mathrm{e})$-octenoyl-[acp]| $\Rightarrow 1 \mid$ nad $\mid+1$ |octanoyl-acp|;78777945 $\backslash \mathrm{n}$ Rxn05326;1 |nadh $|+1| \mathrm{h}+|+1|(2 \mathrm{e})$-hexenoyl-[acp]| $>1$ nad $\mid+1$ |hexanoyl-acp|;78777945 $\mid \mathrm{n}$ Rxn05327;1 |nadh $|+1| \mathrm{h}+|+1|(2 \mathrm{e})$-decenoyl-[acp]| $\Rightarrow 1 \mid$ nad $|+1|$ decanoyl-acp $\mid ; 78777945 \backslash \mathrm{n}$ Rxn05328;1 |nadh $|+1| \mathrm{h}+|+1|$ (2e)-hexadecenoyl-[acp] $|\Rightarrow 1|$ nad $\mid+1$ |hexadecanoyl-acp I; $78777945 \backslash \mathrm{n}$

rxn05329;1 |( r )-3-hydroxybutanoyl-[acyl-carrier protein ] $\Leftrightarrow 1 \mid$ h2o | $+1 \mid$ but-2-enoyl-[acylcarrier protein]|; $78777659 \backslash \mathrm{n}$

rxn05330;1 |d-3-hydroxyhexanoyl-[acp] $\Leftrightarrow 1 \mid$ h2o $|+1 \quad|(2$ e)-hexenoyl-[acp]|;78777659 $\backslash \mathrm{n}$ rxn05331;1 |d-3-hydroxydodecanoyl-[acp]| $\Leftrightarrow 1 \mid$ h2o $|+1|(2$ e)-dodecenoyl-[acp]|;78777659 $\backslash \mathrm{n}$ rxn05332;1 |r-3-hydroxypalmitoyl-acyl-carrierprotein $-|\Leftrightarrow 1|$ h $20|+1|(2$ e) -hexadecenoyl-[acp ]1; $78777659 \backslash \mathrm{n}$

rxn05333;1 |(r)-3-hydroxydecanoyl-[acyl-carrier protein $]|\Longleftrightarrow 1|$ h2o $|+1|(2$ e) - decenoyl-[acp ]|; $78777659 \backslash \mathrm{n}$

rxn05334;1 |( r)-3-hydroxyoctanoyl-[acyl-carrier protein ] $\Leftrightarrow 1 \mid$ h2o | +1 (2e)-octenoyl-[acp $11 ; 78777659 \backslash \mathrm{n}$

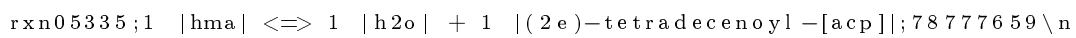

Rxn05336;1 |nadph $|+1| \mathrm{h}+|+1| 3$-oxohexadecanoyl-acp $|\Leftrightarrow 1|$ nadp $|+1| \mathrm{r}-3-\mathrm{hydroxypalmitoyl-}$ acyl-carrierprotein $-1 ; 78777810,78777425 \backslash \mathrm{n}$

Rxn05337;1 |nadph $|+1| \mathrm{h}+|+1| 3$-oxohexanoyl-[acp] $|\Longleftrightarrow 1|$ nadp $\mid+1$ |d-3-hydroxyhexanoyl-[acp ]|; $78777810,78777425 \backslash \mathrm{n}$

Rxn05338;1 |nadph $|+1| \mathrm{h}+|+1| 3$-oxodecanoyl-acp $|\Leftrightarrow 1|$ nadp $|+1|$ (r) -3-hydroxydecanoyl-[acylcarrier protein]|; $78777810,78777425 \backslash \mathrm{n}$

Rxn05339;1 |nadph $|+1| \mathrm{h}+|+1|$ acetoacetyl-acp $|\Leftrightarrow 1|$ nadp $|+1|$ ( $\mathrm{r}$ ) -3-hydroxybutanoyl-[acylcarrier protein]|; $78777810,78777425 \backslash \mathrm{n}$

Rxn05340;1 |nadph $|+1| \mathrm{h}+|+1| 3$-oxododecanoyl-acp $|\Leftrightarrow 1|$ nadp $|+1| d-3-h y d r o x y d o d e c a n o y l-[a c p$ ]|; $78777810,78777425 \backslash \mathrm{n}$

Rxn05341;1 |nadph $|+1| \mathrm{h}+|+1| 3$-oxooctanoyl-acp $|\Leftrightarrow 1|$ nadp $|+1|($ r) $-3-h y d r o x y o c t a n o y l-[a c y l-$ carrier protein]|; $78777810,78777425 \backslash \mathrm{n}$

Rxn05342;1 |nadph $|+1| \mathrm{h}+|+1| 3$-oxotetradecanoyl-acp $|\Longleftrightarrow 1|$ nadp $\mid+1$ |hma|;78777810,78777425 $\mathrm{n}$

rxn05343;1 $|\mathrm{h}+|+1 \mid$ octanoyl-acp $\mid+1$ |malonyl-acyl-carrierprotein $-|\Rightarrow 1|$ co $2|+1| 3-$ oxodecanoyl-acp| + 1 |acp|;78777965,78777806\n

rxn05344;1 $|\mathrm{h}+|+1 \mid$ myristoyl-acp $|+1|$ malonyl-acyl-carrierprotein $-|\Rightarrow 1| \operatorname{co} 2|+1| 3-$ oxohexadecanoyl-acp| + 1 acp|;78777965,78777806\n

rxn05345;1 $|\mathrm{h}+|+1 \mid$ dodecanoyl-acp $|+1|$ malonyl-acyl-carrierprotein $-|\Rightarrow 1| c o 2|+1| 3-$ oxotetradecanoyl-acp| + 1 |acp|;78777965,78777806\n

rxn05346;1 |h+l + 1 |butyryl-acp $|+1|$ malonyl-acyl-carrierprotein $-|\Rightarrow 1| \operatorname{co} 2|+1| 3-o x o h e x a n o y l$ $-[\operatorname{acp}]|+1| \operatorname{acp} \mid ; 78777965,78777806 \backslash \mathrm{n}$

rxn05347;1 |h+l + 1 | malonyl-acyl-carrierprotein $-|+1|$ acetyl-acp $|\Rightarrow 1| \operatorname{co} 2|+1|$ acetoacetylacp| + 1 acp|;78777965,78777806\n

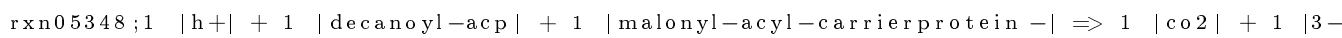
oxododecanoyl-acp| + 1 |acp|;78777965,78777806\n

$\operatorname{rxn05349;1} \mid$ acetyl-coa $|+1| \operatorname{acp}|\Leftrightarrow 1|$ coa $|+1|$ acetyl-acp|;78777965,78777806\n

$\operatorname{rxn} 05350 ; 1|\mathrm{~h}+|+1 \mid$ hexanoyl-acp $|+1|$ malonyl-acyl-carrierprotein $-|\Rightarrow 1| \operatorname{co} 2|+1| 3-$ oxooctanoyl-acp| + 1 |acp|;78777965,78777806\n

rxn05358;1 $\mid 2$-methylbutyryl-coa $|+1|$ acp $|\Leftrightarrow 1|$ coa $|+1| 2$-methylbutyryl-acp|;78777590 $\backslash \mathrm{n}$ rxn05359;1 |h+l + 1 | malonyl-acyl-carrierprotein -| $+1 \mid 2$-methylbutyryl-acp $|\Rightarrow 1| \operatorname{co} 2|+1|$ acp $\mid$ +1 |4-methyl-3-oxo-hexanoyl-acp|; 78777965,78777806\n

rxn05360;1 |nadph $|+1| \mathrm{h}+|+1| 4-$ methyl-3-oxo-hexanoyl-acp $|<\Leftrightarrow 1|$ nadp $|+1| 4-$ methyl-3-hydroxy -hexanoyl-acp|; $78777810,78777425 \backslash \mathrm{n}$

rxn05361; $\mid 4-$ methyl-3-hydroxy-hexanoyl-acp $|\Leftrightarrow 1|$ h2o $|+1| 4-$ methyl-trans-hex-2-enoyl-acp ।;78777659 \n

$\operatorname{rxn05363;1} \mid \mathrm{h}+\mathrm{I}+1$ |malonyl-acyl-carrierprotein $-|+1| 4$-methyl-hexanoyl-acp $|\Rightarrow 1| \operatorname{co} 2|+1|$ acp $|+1| 6$-methyl-3-oxo-octanoyl-acp|;78777965,78777806\n

rxn05364;1 $\mid$ nadph $|+1| \mathrm{h}+|+1| 6$-methyl-3-oxo-octanoyl-acp $|<\Leftrightarrow|$ nadp $|+1| 6-$ methyl-3-hydroxy -octanoyl-acp|; $78777810,78777425 \backslash \mathrm{n}$

rxn05365; $\mid 6$-methyl-3-hydroxy-octanoyl-acp $|\Leftrightarrow 1|$ h2o $|+1| 6$-methyl-trans-oct-2-enoyl-acp 1;78777659\n 
$\operatorname{rxn05367;1}|\mathrm{h}+\mathrm{l}+1|$ malonyl-acyl-carrierprotein $-|+1| 6$-methyl-octanoyl-acp $|\Rightarrow 1| \operatorname{co} 2|+1|$ acp

$|+1| 8$-methyl-3-oxo-decanoyl-acp|;78777965,78777806\n

rxn05368;1 |nadph $|+1| \mathrm{h}+|+1| 8$-methyl-3-oxo-decanoyl-acp $|\Longleftrightarrow 1|$ nadp $|+1| 8-$ methyl-3-hydroxy -decanoyl-acp|; $78777810,78777425 \backslash \mathrm{n}$

rxn05369;1 |8-methyl-3-hydroxy-decanoyl-acp $|\Leftrightarrow 1|$ h2o $|+1| 8-$ methyl-trans-dec-2-enoyl-acp I; $78777659 \backslash \mathrm{n}$

$\operatorname{rxn05371;1}|\mathrm{h}+|+1 \mid$ malonyl-acyl-carrierprotein $-|+1| 8$-methyl-decanoyl-acp $|\Rightarrow 1| \operatorname{co} 2|+1|$ acp

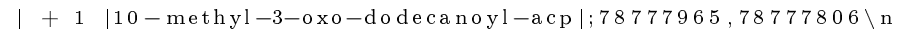

$\operatorname{rxn05372;1} \mid$ nadph $|+1| \mathrm{h}+|+1| 10-$ methyl-3-oxo-dodecanoyl-acp $|<=1|$ nadp $|+1| 10-$ methyl-3hydroxy-dodecanoyl-acp|; $78777810,78777425 \backslash \mathrm{n}$

rxn05373;1 |10-methyl-3-hydroxy-dodecanoyl-acp $|\Leftrightarrow 1|$ h2o $|+1| 10-$ methyl-trans-dodec-2-enoyl-acp $1 ; 78777659 \backslash \mathrm{n}$

$\operatorname{rxn05375;1} \mid \mathrm{h}+\mathrm{|}+1$ | malonyl-acyl-carrierprotein $-|+1| 10$-methyl-dodecanoyl-acp $|\Rightarrow 1| \operatorname{co} 2|+1|$

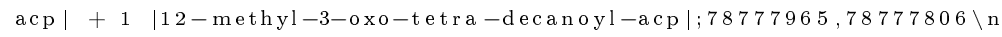

rxn05376;1 |nadph $|+1| \mathrm{h}+|+1| 12$-methyl-3-oxo-tetra-decanoyl-acp $|<1|$ nadp $|+1| 12-$ methyl -3-hydroxy-tetra-decanoyl-acp|; $78777810,78777425 \backslash \mathrm{n}$

rxn05377;1 |12-methyl-3-hydroxy-tetra-decanoyl-acp| $\Leftrightarrow 1||$ h2o $|+1| 12$-methyl-trans-tetra-dec-2enoyl-acp|; $78777659 \backslash \mathrm{n}$

$\operatorname{rxn05379;1}|\mathrm{h}+\mathrm{I}+1|$ malonyl-acyl-carrierprotein $-|+1| 12$-methyl-tetra-decanoyl-acp $|\Rightarrow 1| c o 2 \mid+$

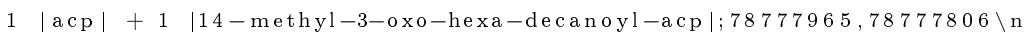

$\operatorname{rxn05380;1} \mid$ nadph $|+1| \mathrm{h}+|+1| 14-$ methyl-3-oxo-hexa-decanoyl-acp $|<\Rightarrow 1|$ nadp $|+1| 14-$ methyl-3hydroxy-hexa-decanoyl-acp|; $78777810,78777425 \backslash \mathrm{n}$

rxn05381; $\mid 14-$ methyl-3-hydroxy-hexa-decanoyl-acp $|\Leftrightarrow 1|$ h2o $|+1| 14-$ methyl-trans-hexa-dec-2enoyl-acp|; $78777659 \backslash \mathrm{n}$

$\operatorname{rxn05383;1} \mid$ isovaleryl-coa $|+1|$ acp $|\Leftrightarrow 1|$ coa $\mid+1$ |isovaleryl-acp|;78777590\n

$\operatorname{rxn05384;1} \mid \mathrm{h}+\mathrm{I}+1$ |malonyl-acyl-carrierprotein $-|+1|$ isovaleryl-acp $|\Rightarrow 1| \operatorname{co} 2|+1|$ acp $\mid+1$

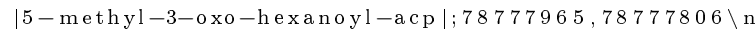

rxn05385; 1 |nadph $|+1| \mathrm{h}+|+1| 5$-methyl-3-oxo-hexanoyl-acp $|<\Rightarrow|$ nadp $|+1| 5-$ methyl-3-hydroxy -hexanoyl-acp|; $78777810,78777425 \backslash \mathrm{n}$

rxn05386;1 |5-methyl-3-hydroxy-hexanoyl-acp $|\Leftrightarrow 1|$ h2o $|+1| 5-$ methyl-trans-hex-2-enoyl-acp I; $78777659 \backslash \mathrm{n}$

$\operatorname{rxn05388;1}|\mathrm{h}+|+1 \mid$ malonyl-acyl-carrierprotein $-|+1| 5$-methyl-hexanoyl-acp $|\Rightarrow 1| \operatorname{co} 2|+1|$ acp $|+1| 7$-methyl-3-oxo-octanoyl-acp|;78777965,78777806\n

rxn05389;1 |nadph $+1|\mathrm{~h}+|+1 \mid 7$-methyl-3-oxo-octanoyl-acp $|<\Rightarrow 1|$ nadp $|+1| 7-$ methyl-3-hydroxy -octanoyl-acp|; $78777810,78777425 \backslash \mathrm{n}$

rxn05390;1 |7-methyl-3-hydroxy-octanoyl-acp $|\Leftrightarrow 1|$ h2o $|+1| 7$-methyl-trans-oct-2-enoyl-acp $1 ; 78777659 \backslash \mathrm{n}$

$\operatorname{rxn05392;1}|\mathrm{h}+|+1 \mid$ malonyl-acyl-carrierprotein $-|+1| 7$-methyl-octanoyl-acp $|\Rightarrow 1| c 02|+1|$ acp | + 1 |9-methyl-3-oxo-decanoyl-acp|;78777965,78777806\n

rxn05393;1 |nadph $|+1| \mathrm{h}+|+1| 9-$ methyl-3-oxo-decanoyl-acp $\mid<\Rightarrow 1$ nadp $|+1| 9-$ methyl-3-hydroxy -decanoyl-acp|; $78777810,78777425 \backslash \mathrm{n}$

rxn05394;1 |9-methyl-3-hydroxy-decanoyl-acp $|\Leftrightarrow 1|$ h2o $|+1| 9-$ methyl-trans-dec-2-enoyl-acp 1; $78777659 \backslash \mathrm{n}$

$\operatorname{rxn05396;1}|\mathrm{h}+|+1 \mid$ malonyl-acyl-carrierprotein $-|+1| 9-$ methyl-decanoyl-acp $|\Rightarrow 1| \operatorname{co} 2|+1|$ acp | + 1 |11-methyl-3-oxo-dodecanoyl-acp|;78777965,78777806\n

rxn05397;1 |nadph $|+1| \mathrm{h}+|+1| 11$-methyl-3-oxo-dodecanoyl-acp $|<\Rightarrow 1|$ nadp $|+1| 11-$ methyl-3hydroxy-dodecanoyl-acp|; $78777810,78777425 \backslash \mathrm{n}$

rxn05398;1 |11-methyl-3-hydroxy-dodecanoyl-acp $|\Leftrightarrow 1|$ h2o $|+1| 11-$ methyl-trans-dodec-2-enoyl-acp $1 ; 78777659 \backslash \mathrm{n}$

rxn05400;1 |h+l + 1 | malonyl-acyl-carrierprotein -| + $1 \mid 11$-methyl-dodecanoyl-acp $\Rightarrow 1|c o 2|+1 \mid$

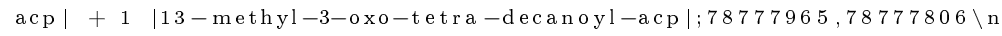

rxn05401;1 |nadph $|+1| \mathrm{h}+|+1| 13$-methyl-3-oxo-tetra-decanoyl-acp $|<1|$ nadp $|+1| 13-$ methyl $-3-$ hydroxy-tetra-decanoyl-acp|; $78777810,78777425 \backslash \mathrm{n}$

rxn05402;1 |13-methyl-3-hydroxy-tetra-decanoyl-acp $|\Leftrightarrow 1| \mid$ h2o $|+1| 13$-methyl-trans-tetra-dec-2enoyl-acp|; $78777659 \backslash \mathrm{n}$

$\operatorname{rxn05404;1}|\mathrm{h}+|+1 \mid$ malonyl-acyl-carrierprotein $-|+1| 13$-methyl-tetra-decanoyl-acp $|\Rightarrow 1| c o 2 \mid+$

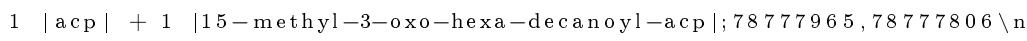

$\operatorname{rxn05405;1} \mid$ nadph $|+1| \mathrm{h}+|+1| 15-$ methyl-3-oxo-hexa-decanoyl-acp $|<\Rightarrow 1|$ nadp $|+1| 15-$ methyl-3hydroxy-hexa-decanoyl-acp|; $78777810,78777425 \backslash \mathrm{n}$

rxn05406;1 |15-methyl-3-hydroxy-hexa-decanoyl-acp $|\Leftrightarrow 1|$ h2o $|+1| 15-$ methyl-trans-hexa-dec-2enoyl-acp|; $78777659 \backslash \mathrm{n}$ 
$\operatorname{rxn05408;1} \mid$ isobutyryl-coa $|+1|$ acp $|\Leftrightarrow 1|$ coa $\mid+1$ |isobutyryl-acp|;78777590 $\Rightarrow \mathrm{n}$

rxn05409;1 |h+| + 1 | malonyl-acyl-carrierprotein $-|+1|$ isobutyryl-acp $|\Rightarrow 1| c o 2|+1| a c p \mid+1$ $\mid 4$-methyl-3-oxo-pentanoyl-acp|;78777965,78777806\n

rxn05410;1 |nadph| $1|\mathrm{~h}+|+1 \mid 4-$ methyl-3-oxo-pentanoyl-acp $|<1|$ nadp $|+1| 4-$ methyl-3hydroxy-pentanoyl-acp|; $78777810,78777425 \backslash \mathrm{n}$

rxn05411;1 $\mid 4-$ methyl-3-hydroxy-pentanoyl-acp $|\Leftrightarrow 1|$ h2o $|+1| 4-$ methyl-trans-pent-2-enoyl-acp I; $78777659 \backslash \mathrm{n}$

rxn05413;1 |h+l + 1 | malonyl-acyl-carrierprotein -| + $1 \mid 4$-methyl-pentanoyl-acp| $\Rightarrow 1|c o 2|+1 \mid$ acp $|+1| 6-$ methyl-3-oxo-heptanoyl-acp|;78777965,78777806\n

rxn05414;1 |nadph| $1|\mathrm{~h}+|+1 \mid 6$-methyl-3-oxo-heptanoyl-acp $|<1|$ nadp $|+1| 6-$ methyl-3hydroxy-heptanoyl-acp|; $78777810,78777425 \backslash \mathrm{n}$

rxn05415;1 |6-methyl-3-hydroxy-heptanoyl-acp $|\Leftrightarrow 1|$ h2o $|+1| 6$-methyl-trans-hept-2-enoyl-acp I; $78777659 \backslash \mathrm{n}$

rxn05417;1 |h+l + 1 | malonyl-acyl-carrierprotein $-|+1| 6$-methyl-heptanoyl-acp $|\Rightarrow 1| \operatorname{co} 2|+1|$ acp | 1 |8-methyl-3-oxo-nonanoyl-acp|;78777965,78777806\n

rxn05418;1 |nadph $|+1| \mathrm{h}+|+1| 8$-methyl-3-oxo-nonanoyl-acp $|<\Rightarrow 1|$ nadp $|+1| 8-$ methyl-3-hydroxy -nonanoyl-acp|; $78777810,78777425 \backslash \mathrm{n}$

rxn05419;1 |8-methyl-3-hydroxy-nonanoyl-acp $|\Leftrightarrow 1|$ h2o $|+1| 8-$ methyl-trans-non-2-enoyl-acp $1 ; 78777659 \backslash \mathrm{n}$

$\operatorname{rxn} 05421 ; 1|\mathrm{~h}+|+1 \mid$ malonyl-acyl-carrierprotein $-|+1| 8$-methyl-nonanoyl-acp $|\Rightarrow 1| \operatorname{co} 2|+1|$ acp | + $1 \mid 10-$ methyl-3-oxo-undecanoyl-acp|;78777965,78777806\n

rxn05422; 1 nadph $|+1| \mathrm{h}+|+1| 10-$ methyl-3-oxo-undecanoyl-acp $|<=1|$ nadp $|+1| 10-$ methyl-3hydroxy-undecanoyl-acp|; $78777810,78777425 \backslash \mathrm{n}$

rxn05423;1 |10-methyl-3-hydroxy-undecanoyl-acp $|\Leftrightarrow 1|$ h2o $|+1| 10-$ methyl-trans-undec-2-enoyl-acp $1 ; 78777659 \backslash \mathrm{n}$

$\operatorname{rxn05425;1}|\mathrm{h}+|+1 \mid$ malonyl-acyl-carrierprotein $-|+1| 10$-methyl-undecanoyl-acp $|\Rightarrow 1| c o 2|+1|$ acp $|+1| 12$-methyl-3-oxo-tridecanoyl-acp|;78777965,78777806\n

$\operatorname{rxn05426;1} \mid$ nadph $|+1| \mathrm{h}+|+1| 12-$ methyl-3-oxo-tridecanoyl-acp $|<=1|$ nadp $|+1| 12-$ methyl-3-

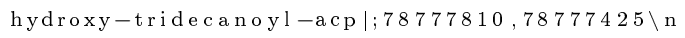

rxn05427;1 |12-methyl-3-hydroxy-tridecanoyl-acp $|<1|$ h2o $|+1| 12-$ methyl-trans-tridec-2-enoylacp |; $78777659 \backslash \mathrm{n}$

rxn05429;1 |h+l + 1 | malonyl-acyl-carrierprotein -| + $1 \mid 12$-methyl-tridecanoyl-acp $|\Rightarrow 1|$ co $2 \mid+1$ $|\operatorname{acp}|+1 \mid 14-$ methyl-3-oxo-pentadecanoyl-acp|;78777965,78777806\n

rxn05430;1 |nadph $+1|\mathrm{~h}+|+1 \mid 14-$ methyl-3-oxo-pentadecanoyl-acp $|<\Rightarrow 1|$ nadp $|+1| 14-$ methyl-3-

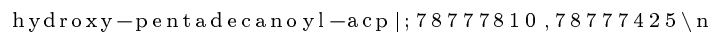

rxn05431;1 |14-methyl-3-hydroxy-pentadecanoyl-acp $|\Leftrightarrow 1|$ h2o $|+1| 14-$ methyl-trans-pentadec-2enoyl-acp|;78777659 \n

rxn05433;1 |nadh| + $1|\mathrm{~h}+|+1 \mid 4-$ methyl-trans-hex-2-enoyl-acp $|\Rightarrow 1|$ nad $|+1| 4-$ methyl-hexanoylacp |; $78777945 \backslash \mathrm{n}$

$\operatorname{rxn05434;1} \mid$ nadh $|+1| \mathrm{h}+|+1| 6$-methyl-trans-oct-2-enoyl-acp $\Rightarrow 1 \mid$ nad $|+1| 6-$ methyl-octanoylacp $\mid ; 78777945 \backslash \mathrm{n}$

rxn05435;1 |nadh $|+1| \mathrm{h}+|+1| 8-$ methyl-trans-dec-2-enoyl-acp $|\Rightarrow 1|$ nad $|+1| 8-$ methyl-decanoylacp|;78777945 \n

$\operatorname{rxn05436;1} \mid$ nadh $|+1| \mathrm{h}+|+1| 10-$ methyl-trans-dodec-2-enoyl-acp $|\Rightarrow 1|$ nad $|+1| 10-$ methyldodecanoyl-acp|;78777945 $\backslash \mathrm{n}$

rxn05437;1 |nadh $|+1| \mathrm{h}+|+1| 12$ - methyl-trans-tetra-dec-2-enoyl-acp $|\Rightarrow 1|$ nad $|+1| 12-$ methyltetra-decanoyl-acp|; $78777945 \backslash \mathrm{n}$

$\operatorname{rxn05438;1} \mid$ nadh $|+1| \mathrm{h}+|+1| 14-$ methyl-trans-hexa-dec-2-enoyl-acp $|\Rightarrow 1|$ nad $|+1| 14-$ methylhexa-decanoyl-acp|;78777945 $\backslash \mathrm{n}$

rxn05439;1 |nadh $|+1| \mathrm{h}+|+1| 5$-methyl-trans-hex-2-enoyl-acp $|\Rightarrow 1|$ nad $|+1| 5-$ methyl-hexanoylacp|; $78777945 \backslash \mathrm{n}$

rxn05440;1 |nadh $|+1| \mathrm{h}+|+1| 7$-methyl-trans-oct-2-enoyl-acp $|\Rightarrow 1|$ nad $|+1| 7-$ methyl-octanoylacp|; $78777945 \backslash \mathrm{n}$

rxn05441;1 |nadh $|+1| \mathrm{h}+|+1| 9-$ methyl-trans-dec-2-enoyl-acp $|\Rightarrow 1|$ nad $|+1| 9-$ methyl-decanoylacp |; $78777945 \backslash \mathrm{n}$

rxn05442;1 |nadh $|+1| \mathrm{h}+|+1| 11-$ methyl-trans-dodec-2-enoyl-acp $|\Rightarrow 1|$ nad $|+1| 11-$ methyldodecanoyl-acp|; $78777945 \backslash \mathrm{n}$

rxn05443;1 |nadh $|+1| \mathrm{h}+|+1| 13$-methyl-trans-tetra-dec-2-enoyl-acp $|\Rightarrow 1|$ nad $|+1| 13-$ methyl-

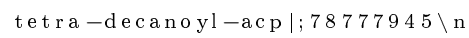

rxn05444;1 |nadh $|+1| \mathrm{h}+|+1| 15-$ methyl-trans-hexa-dec-2-enoyl-acp $|\Rightarrow 1|$ nad $|+1| 15-m e t h y l-$ hexa-decanoyl-acp|;78777945 \n 


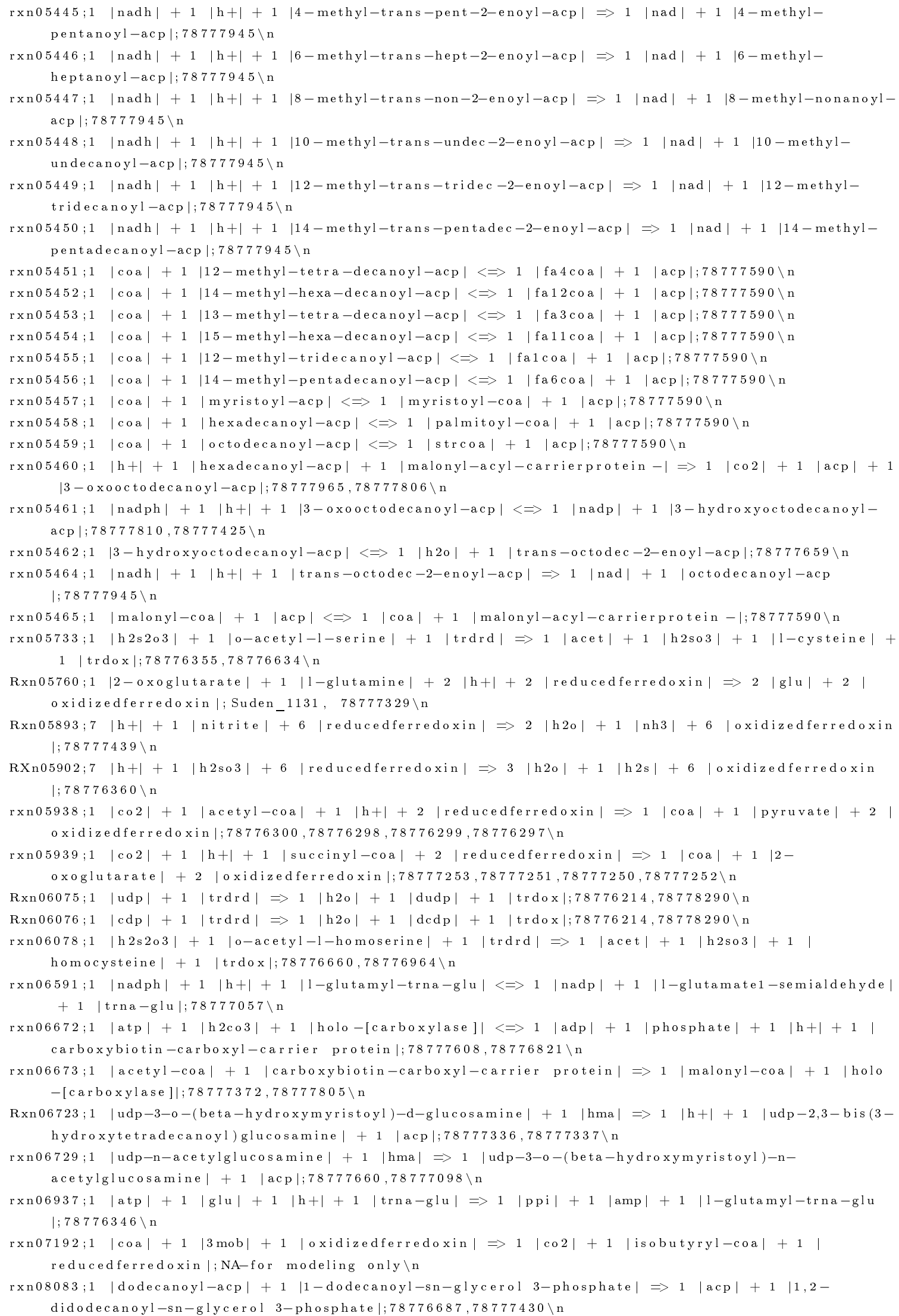




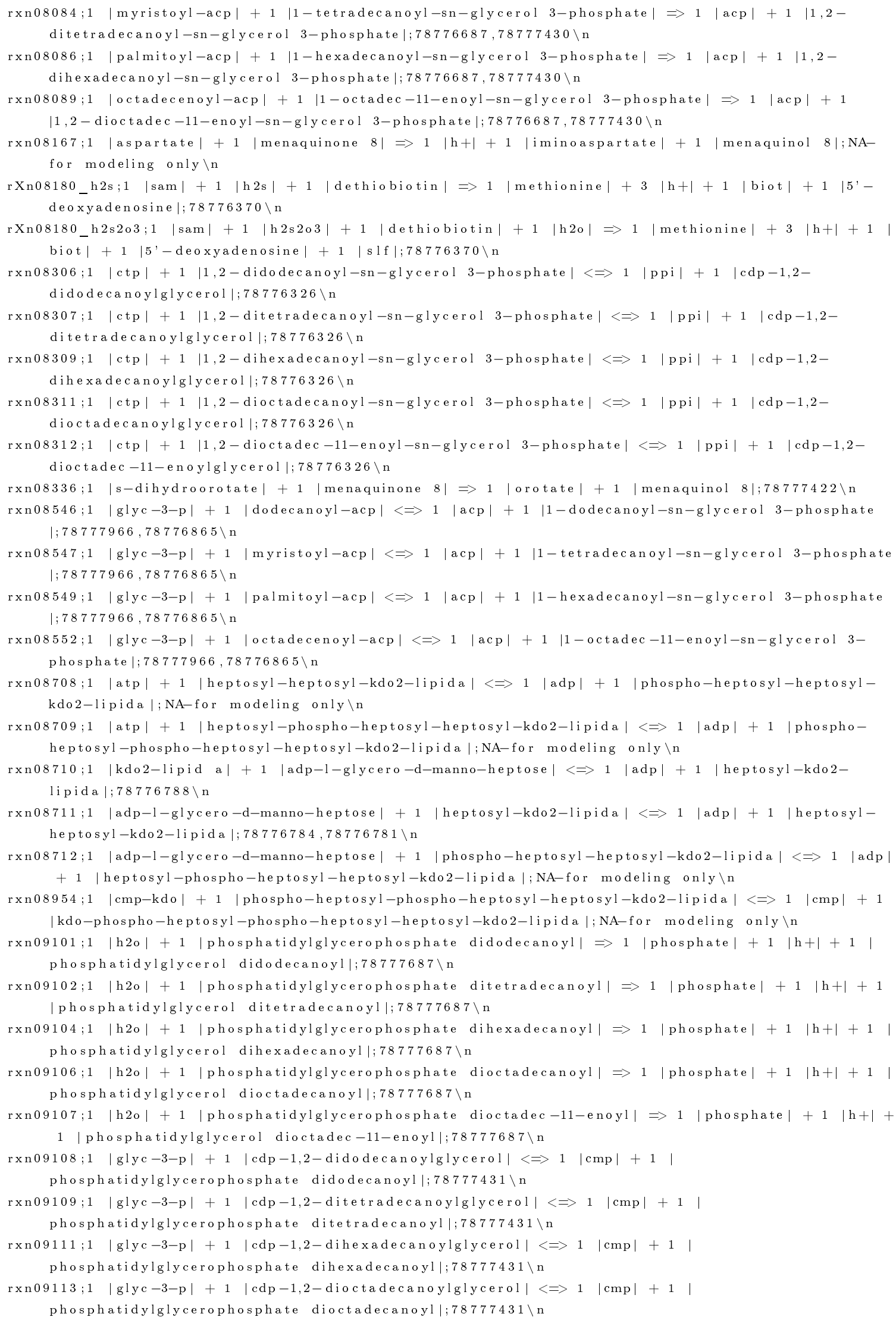




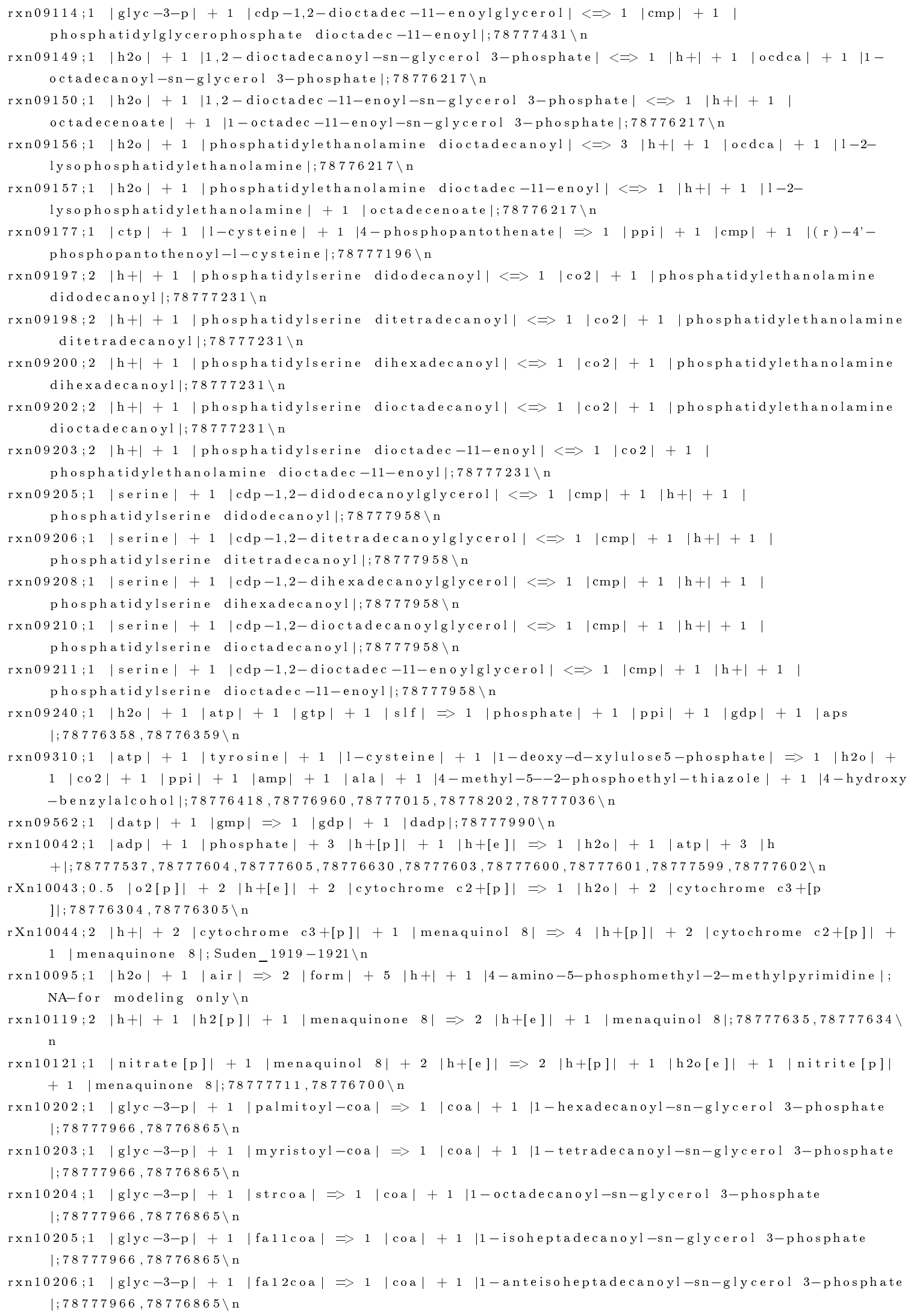




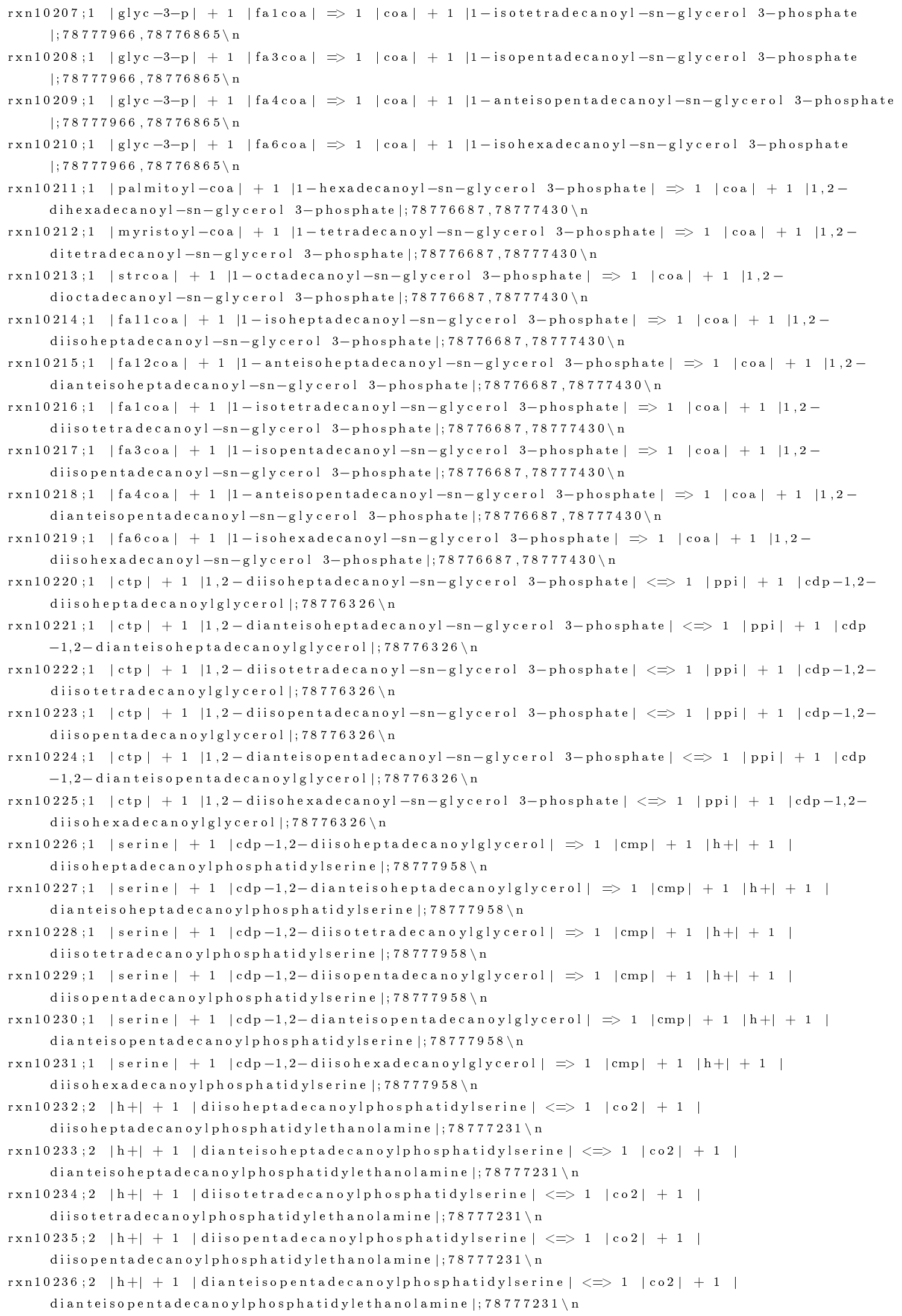




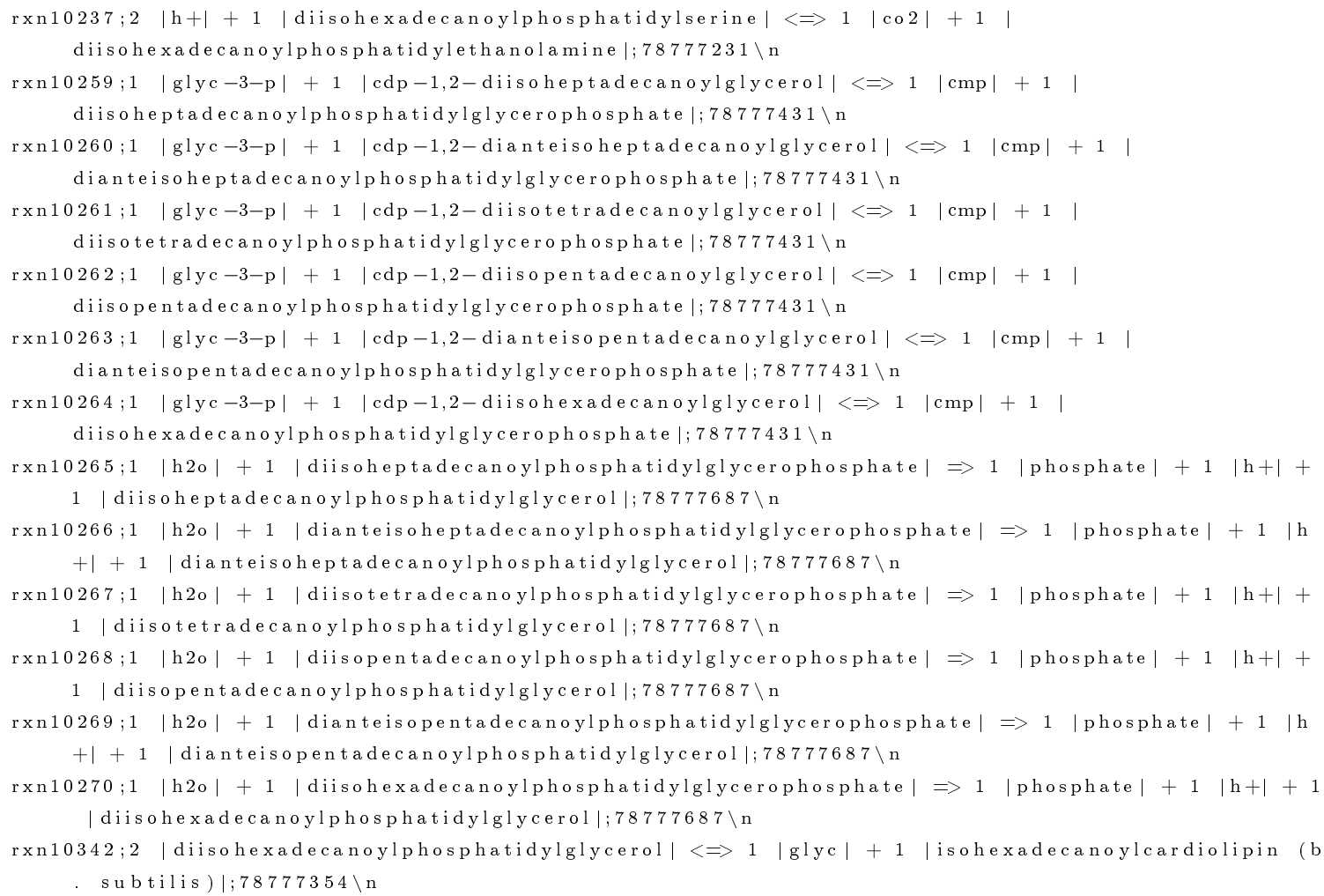




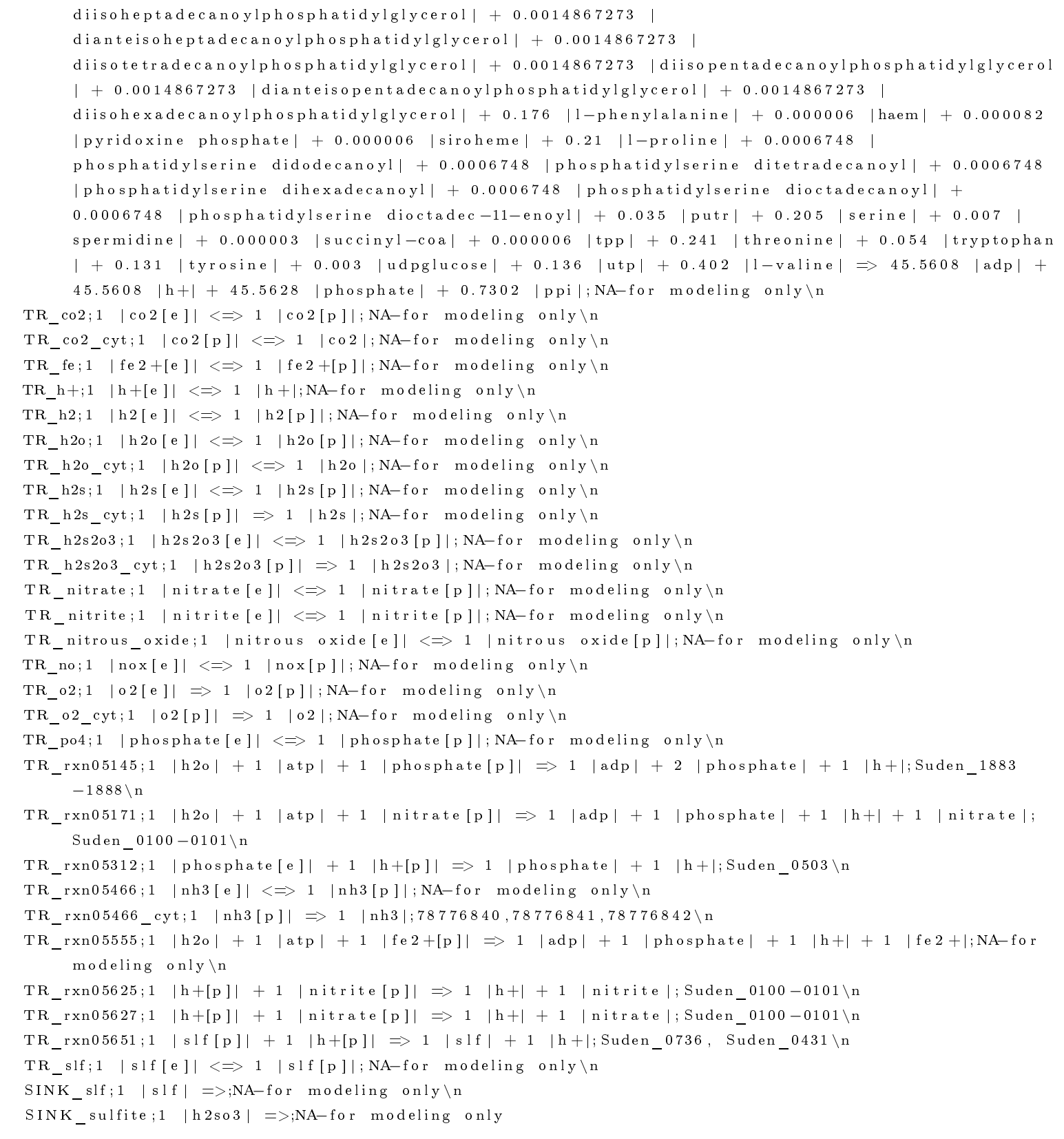




\section{Bibliography}

Al-Haideri, H., White, M. A., and Kelly, D. J. (2016). Major contribution of the type II beta carbonic anhydrase CanB (Cj0237) to the capnophilic growth phenotype of Campylobacter jejuni. Environmental Microbiology, 18(2):721-735.

Amann, R. and Moraru, C. (2012). Two decades of fluorescence in situ hybridization in systematic and applied microbiology. Systematic and Applied Microbiology, 35(8):483-484.

Amann, R. I., Binder, B. J., Olson, R. J., Chisholm, S. W., Devereux, R., and Stahl, D. A. (1990). Combination of 16s rRNA-targeted oligonucleotide probes with flow cytometry for analyzing mixed microbial populations. Applied and Environmental Microbiology, 56(6):1919-1925.

Amend, J. P., McCollom, T. M., Hentscher, M., and Bach, W. (2011). Catabolic and anabolic energy for chemolithoautotrophs in deep-sea hydrothermal systems hosted in different rock types. Geochimica et Cosmochimica Acta, 75(19):5736-5748.

Anantharaman, K., Breier, J. A., and Dick, G. J. (2016). Metagenomic resolution of microbial functions in deep-sea hydrothermal plumes across the Eastern Lau Spreading Center. The ISME Journal, 10(1):225-239.

Anderson, R. E., Torres, B. M., J, H. S., and A, B. J. (2013). Microbial community structure across fluid gradients in the Juan de Fuca Ridge hydrothermal system. FEMS Microbiology Ecology, 83(2):324-339.

Ballard, R. D. (1977). Notes on a major oceanographic find (marine animals near hot-water vents at ocean bottom). Oceanus, 20:35-44.

Bartlett, D. H. (2002). Pressure effects on in vivo microbial processes. Biochimica et Biophysica Acta (BBA) - Protein Structure and Molecular Enzymology, 1595(12):367-381.

Berg, I. A. (2011). Ecological Aspects of the Distribution of Different Autotrophic $\mathrm{CO}_{2}$ Fixation Pathways. Applied and Environmental Microbiology, 77(6):1925-1936.

Berg, J. S., Schwedt, A., Kreutzmann, A.-C., Kuypers, M. M. M., and Milucka, J. (2014). Polysulfides as Intermediates in the Oxidation of Sulfide to Sulfate by Beggiatoa spp. Applied and Environmental Microbiology, 80(2):629-636.

Bianchi, A., Garcin, J., and Tholosan, O. (1999). A high-pressure serial sampler to measure microbial activity in the deep sea. Deep Sea Research Part I: Oceanographic Research Papers, 46(12):2129-2142. 
Bolton, F. J. and Coates, D. (1983). A study of the oxygen and carbon dioxide requirements of thermophilic campylobacters. Journal of Clinical Pathology, 36(7):829-834.

Bourbonnais, A., Juniper, S. K., Butterfield, D. A., Devol, A. H., Kuypers, M. M. M., Lavik, G., Hallam, S. J., Wenk, C. B., Chang, B. X., Murdock, S. A., and Lehmann, M. F. (2012). Activity and abundance of denitrifying bacteria in the subsurface biosphere of diffuse hydrothermal vents of the Juan de Fuca Ridge. Biogeosciences, 9(11):4661-4678.

Brondijk, T., Nilavongse, A., Filenko, N., Richardson, D., and Cole, J. (2004). NapGH components of the periplasmic nitrate reductase of Escherichia coli K-12: location, topology and physiological roles in quinol oxidation and redox balancing. Biochemical Journal, 379(1):47. 00056.

Bruscella, P., Appia-Ayme, C., Levican, G., Ratouchniak, J., Jedlicki, E., Holmes, D. S., and Bonnefoy, V. (2007). Differential expression of two bc1 complexes in the strict acidophilic chemolithoautotrophic bacterium Acidithiobacillus ferrooxidans suggests a model for their respective roles in iron or sulfur oxidation. Microbiology, 153(1):102-110.

Butterfield, D. A., Roe, K. K., Lilley, M. D., Huber, J. A., Baross, J. A., Embley, R. W., and Massoth, G. J. (2004). Mixing, Reaction and Microbial Activity in the Sub-Seafloor Revealed by Temporal and Spatial Variation in Diffuse Flow Vents at Axial Volcano. In Wilcock, W. S. D., Delong, E. F., Kelley, D. S., Baross, J. A., and Cary, S. C., editors, The Subseafloor Biosphere at Mid-Ocean Ridges, pages 269-289. American Geophysical Union.

Cai, L., Shao, M.-F., and Zhang, T. (2014). Non-contiguous finished genome sequence and description of Sulfurimonas hongkongensis sp. nov., a strictly anaerobic denitrifying, hydrogen- and sulfur-oxidizing chemolithoautotroph isolated from marine sediment. Standards in Genomic Sciences, 9(3):1302-1310.

Campbell, B. J. and Cary, S. C. (2004). Abundance of reverse tricarboxylic acid cycle genes in free-living microorganisms at deep-sea hydrothermal vents. Applied and Environmental Microbiology, 70(10):6282-6289.

Campbell, B. J., Engel, A. S., Porter, M. L., and Takai, K. (2006). The versatile Epsilonproteobacteria: key players in sulphidic habitats. Nature Reviews Microbiology, 4(6):458-468.

Campbell, B. J., Jeanthon, C., Kostka, J. E., Luther, G. W., and Cary, S. C. (2001). Growth and Phylogenetic Properties of Novel Bacteria Belonging to the Epsilon Subdivision of the Proteobacteria Enriched from Alvinella pompejana and Deep-Sea Hydrothermal Vents. Applied and Environmental Microbiology, 67(10):4566-4572. Cited by 0101.

Campbell, B. J., Smith, J. L., Hanson, T. E., Klotz, M. G., Stein, L. Y., Lee, C. K., Wu, D., Robinson, J. M., Khouri, H. M., Eisen, J. A., and Cary, S. C. (2009). Adaptations to Submarine Hydrothermal Environments Exemplified by the Genome of Nautilia profundicola. PLoS Genetics, 5(2):e1000362.

Canfield, D. E., Erik Kristensen, and Bo Thamdrup (2005). Thermodynamics and Microbial Metabolism. In Donald E. Canfield, E. K. a. B. T., editor, Advances in Marine Biology, volume Volume 48, pages 65-94. Academic Press. 
Caporaso, J. G., Kuczynski, J., Stombaugh, J., Bittinger, K., Bushman, F. D., Costello, E. K., Fierer, N., Peña, A. G., Goodrich, J. K., Gordon, J. I., Huttley, G. A., Kelley, S. T., Knights, D., Koenig, J. E., Ley, R. E., Lozupone, C. A., McDonald, D., Muegge, B. D., Pirrung, M., Reeder, J., Sevinsky, J. R., Turnbaugh, P. J., Walters, W. A., Widmann, J., Yatsunenko, T., Zaneveld, J., and Knight, R. (2010). QIIME allows analysis of highthroughput community sequencing data. Nature Methods, 7(5):335-336.

Charlou, J. L., Donval, J. P., Douville, E., Jean-Baptiste, P., Radford-Knoery, J., Fouquet, Y., Dapoigny, A., and Stievenard, M. (2000). Compared geochemical signatures and the evolution of Menez Gwen ( $\left.37^{\circ} 50^{\prime} \mathrm{N}\right)$ and Lucky Strike ( $\left.37^{\circ} 17^{\prime} \mathrm{N}\right)$ hydrothermal fluids, south of the Azores Triple Junction on the Mid-Atlantic Ridge. Chemical Geology, 171(12):49-75.

Chen, J. and Strous, M. (2013). Denitrification and aerobic respiration, hybrid electron transport chains and co-evolution. Biochimica et Biophysica Acta (BBA) - Bioenergetics, 1827(2):136-144. Cited by 0000.

Codispoti, L. A. (2007). An oceanic fixed nitrogen sink exceeding $400 \mathrm{Tg} \mathrm{N} \mathrm{a}^{-1}$ vs the concept of homeostasis in the fixed-nitrogen inventory. Biogeosciences, 4(2):233-253.

Corliss, J. B., Dymond, J., Gordon, L. I., Edmond, J. M., von Herzen, R. P., Ballard, R. D., Green, K., Williams, D., Bainbridge, A., Crane, K., and van Andel, T. H. (1979). Submarine thermal sprirngs on the galapagos rift. Science (New York, N.Y.), 203(4385):1073-1083.

Corre, E., Reysenbach, A.-L., and Prieur, D. (2001). $\varepsilon$-Proteobacterial diversity from a deep-sea hydrothermal vent on the Mid-Atlantic Ridge. FEMS Microbiology Letters, 205(2):329-335.

Dahl, C., Friedrich, C., and Kletzin, A. (2008). Sulfur Oxidation in Prokaryotes. In John Wiley \& Sons, Ltd, editor, Encyclopedia of Life Sciences. John Wiley \& Sons, Ltd, Chichester, UK.

Dahle, H., Økland, I., Thorseth, I. H., Pederesen, R. B., and Steen, I. H. (2015). Energy landscapes shape microbial communities in hydrothermal systems on the Arctic MidOcean Ridge. The ISME Journal, 9(7):1593-1606.

Daims, H., Brühl, A., Amann, R., Schleifer, K.-H., and Wagner, M. (1999). The Domainspecific Probe EUB338 is Insufficient for the Detection of all Bacteria: Development and Evaluation of a more Comprehensive Probe Set. Systematic and Applied Microbiology, $22(3): 434-444$.

DeLong, E., Wickham, G., and Pace, N. (1989). Phylogenetic stains: ribosomal RNA-based probes for the identification of single cells. Science, 243(4896):1360 -1363. Cited by 1155 .

Demmer, J. K., Huang, H., Wang, S., Demmer, U., Thauer, R. K., and Ermler, U. (2015). Insights into Flavin-based Electron Bifurcation via the NADH-dependent Reduced Ferredoxin:NADP Oxidoreductase Structure. Journal of Biological Chemistry, 290(36):21985-21995.

Devoid, S., Overbeek, R., DeJongh, M., Vonstein, V., Best, A., and Henry, C. (2013). Automated Genome Annotation and Metabolic Model Reconstruction in the SEED and Model SEED. In Alper, H. S., editor, Systems Metabolic Engineering, number 985 in Methods in Molecular Biology, pages 17-45. Humana Press. DOI: 10.1007/978-1-62703-299-5_2. 
Distel, D. L., Lane, D. J., Olsen, G. J., Giovannoni, S. J., Pace, B., Pace, N. R., Stahl, D. A., and Felbeck, H. (1988). Sulfur-oxidizing bacterial endosymbionts: analysis of phylogeny and specificity by 16s rRNA sequences. Journal of Bacteriology, 170(6):2506-2510.

Dobrinski, K. P., Boller, A. J., and Scott, K. M. (2010). Expression and Function of Four Carbonic Anhydrase Homologs in the Deep-Sea Chemolithoautotroph Thiomicrospira crunogena. Applied and Environmental Microbiology, 76(11):3561-3567.

Ducluzeau, A.-L., van Lis, R., Duval, S., Schoepp-Cothenet, B., Russell, M. J., and Nitschke, W. (2009). Was nitric oxide the first deep electron sink? Trends in Biochemical Sciences, $34(1): 9-15$.

Durot, M., Bourguignon, P.-Y., and Schachter, V. (2009). Genome-scale models of bacterial metabolism: reconstruction and applications. FEMS Microbiology Reviews, 33(1):164-190.

Dworkin, M. (2012). Sergei Winogradsky: a founder of modern microbiology and the first microbial ecologist. FEMS Microbiology Reviews, 36(2):364-379.

Elderfield, H. and Schultz, a. A. (1996). Mid-Ocean Ridge Hydrothermal Fluxes and the Chemical Composition of the Ocean. Annual Review of Earth and Planetary Sciences, 24(1):191-224.

Engel, A. S., Porter, M. L., Stern, L. A., Quinlan, S., and Bennett, P. C. (2004). Bacterial diversity and ecosystem function of filamentous microbial mats from aphotic (cave) sulfidic springs dominated by chemolithoautotrophic Epsilonproteobacteria. FEMS Microbiology Ecology, 51(1):31-53.

Esposti, M. D. (2016). Genome Analysis of StructureFunction Relationships in Respiratory Complex I, an Ancient Bioenergetic Enzyme. Genome Biology and Evolution, $8(1): 126-147$.

Evans, M. C., Buchanan, B. B., and Arnon, D. I. (1966). A new ferredoxin-dependent carbon reduction cycle in a photosynthetic bacterium. Proceedings of the National Academy of Sciences of the United States of America, 55(4):928-934.

Falkowski, P. G. and Raven, J. A. (2013). Aquatic Photosynthesis: (Second Edition). Princeton University Press.

Felbeck, H. (1981). Chemoautotrophic Potential of the Hydrothermal Vent Tube Worm, Riftia pachyptila Jones (Vestimentifera). Science, 213(4505):336-338.

Finn, R. D., Coggill, P., Eberhardt, R. Y., Eddy, S. R., Mistry, J., Mitchell, A. L., Potter, S. C., Punta, M., Qureshi, M., Sangrador-Vegas, A., Salazar, G. A., Tate, J., and Bateman, A. (2016). The Pfam protein families database: towards a more sustainable future. Nucleic Acids Research, 44(D1):D279-D285.

Finster, K., Liesack, W., and Tindall, B. J. (1997). Sulfurospirillum arcachonense sp. nov., a new microaerophilic sulfur-reducing bacterium. International Journal of Systematic Bacteriology, 47(4):1212-1217.

Flores, G. E., Campbell, J. H., Kirshtein, J. D., Meneghin, J., Podar, M., Steinberg, J. I., Seewald, J. S., Tivey, M. K., Voytek, M. A., Yang, Z. K., and Reysenbach, A.-L. (2011). 
Microbial community structure of hydrothermal deposits from geochemically different vent fields along the Mid-Atlantic Ridge: Microbial communities of hydrothermal vent deposits. Environmental Microbiology, 13(8):2158-2171.

Flores, G. E., Wagner, I. D., Liu, Y., and Reysenbach, A.-L. (2012). Distribution, Abundance, and Diversity Patterns of the Thermoacidophilic Deep-Sea Hydrothermal Vent Euryarchaeota 2. Frontiers in Microbiology, 3.

Fortunato, C. S. and Huber, J. A. (2016). Coupled RNA-SIP and metatranscriptomics of active chemolithoautotrophic communities at a deep-sea hydrothermal vent. The ISME Journal.

Foustoukos, D. I., Houghton, J. L., Seyfried Jr., W. E., Sievert, S. M., and Cody, G. D. (2011). Kinetics of $\mathrm{H}_{2} \mathrm{O}_{2} \mathrm{H}_{2} \mathrm{O}$ redox equilibria and formation of metastable $\mathrm{H}_{2} \mathrm{O}_{2}$ under low temperature hydrothermal conditions. Geochimica et Cosmochimica Acta, 75(6):1594-1607.

Fraser, A. D. E., Chandan, V., Yamazaki, H., Brooks, B. W., and Garcia, M. M. (1992). Simple and economical culture of Campylobacter jejuni and Campylobacter coli in $\mathrm{CO}_{2}$ in moist air. International Journal of Food Microbiology, 15(3):377-382.

Friedrich, C. G., Rother, D., Bardischewsky, F., Quentmeier, A., and Fischer, J. (2001). Oxidation of Reduced Inorganic Sulfur Compounds by Bacteria: Emergence of a Common Mechanism? Applied and Environmental Microbiology, 67(7):2873-2882.

Gao, H., Schreiber, F., Collins, G., Jensen, M. M., Kostka, J. E., Lavik, G., de Beer, D., Zhou, H.-y., and Kuypers, M. M. M. (2009). Aerobic denitrification in permeable Wadden Sea sediments. The ISME Journal, 4(3):417-426. Cited by 0039.

Garrity, G. M., Bell, J. A., and Lilburn, T. (2015). Campylobacterales ord. nov. In Bergey's Manual of Systematics of Archaea and Bacteria. John Wiley \& Sons, Ltd.

Garside, C. (1982). A chemiluminescent technique for the determination of nanomolar concentrations of nitrate and nitrite in seawater. Marine Chemistry, 11(2):159-167.

Germanovich, L. N., Hurt, R. S., Smith, J. E., Genc, G., and Lowell, R. P. (2015). Measuring fluid flow and heat output in seafloor hydrothermal environments. Journal of Geophysical Research: Solid Earth, 120(12):8031-8055.

Giovannelli, D., Ferriera, S., Johnson, J., Kravitz, S., Pérez-Rodríguez, I., Ricci, J., OBrien, C., Voordeckers, J. W., Bini, E., and Vetriani, C. (2011). Draft genome sequence of Caminibacter mediatlanticus strain TB-2t, an epsilonproteobacterium isolated from a deep-sea hydrothermal vent. Standards in Genomic Sciences, 5(1):135-143.

Goris, T., Schubert, T., Gadkari, J., Wubet, T., Tarkka, M., Buscot, F., Adrian, L., and Diekert, G. (2014). Insights into organohalide respiration and the versatile catabolism of Sulfurospirillum multivorans gained from comparative genomics and physiological studies. Environmental Microbiology, 16(11):3562-3580.

Greuter, D., Loy, A., Horn, M., and Rattei, T. (2016). probeBasean online resource for rRNA-targeted oligonucleotide probes and primers: new features 2016. Nucleic Acids Research, 44(D1):D586-D589. 
Griesbeck, C., Schütz, M., Schödl, T., Bathe, S., Nausch, L., Mederer, N., Vielreicher, M., and Hauska, G. (2002). Mechanism of Sulfide-Quinone Reductase Investigated Using Site-Directed Mutagenesis and Sulfur Analysis,. Biochemistry, 41(39):11552-11565.

Grosche, A., Sekaran, H., Pérez-Rodríguez, I., Starovoytov, V., and Vetriani, C. (2015). Cetia pacifica gen. nov., sp. nov., a chemolithoautotrophic, thermophilic, nitrateammonifying bacterium from a deep-sea hydrothermal vent. International Journal of Systematic and Evolutionary Microbiology, 65(4):1144-1150.

Gross, R., Simon, J., Theis, F., and Kröger, A. (1998). Two membrane anchors of Wolinella succinogenes hydrogenase and their function in fumarate and polysulfide respiration. Archives of Microbiology, 170(1):50-58.

Grote, J., Jost, G., Labrenz, M., Herndl, G. J., and Jürgens, K. (2008). Epsilonproteobacteria Represent the Major Portion of Chemoautotrophic Bacteria in Sulfidic Waters of Pelagic Redoxclines of the Baltic and Black Seas. Applied and Environmental Microbiology, 74(24):7546-7551.

Grote, J., Schott, T., Bruckner, C. G., Glöckner, F. O., Jost, G., Teeling, H., Labrenz, M., and Jürgens, K. (2012). Genome and physiology of a model Epsilonproteobacterium responsible for sulfide detoxification in marine oxygen depletion zones. Proceedings of the National Academy of Sciences, 109(2):506-510.

Gruber, N. (2004). The Dynamics of the Marine Nitrogen Cycle and its Influence on Atmospheric $\mathrm{CO}_{2}$ Variations. In Follows, M. and Oguz, T., editors, The Ocean Carbon Cycle and Climate, number 40 in NATO Science Series, pages 97-148. Springer Netherlands. DOI: $10.1007 / 978-1-4020-2087-2 \_4$.

Haddad, A., Camacho, F., Durand, P., and Cary, S. C. (1995). Phylogenetic characterization of the epibiotic bacteria associated with the hydrothermal vent polychaete Alvinella pompejana. Applied and Environmental Microbiology, 61(5):1679-1687.

Hagström, Larsson, U., Hörstedt, P., and Normark, S. (1979). Frequency of Dividing Cells, a New Approach to the Determination of Bacterial Growth Rates in Aquatic Environments. Applied and Environmental Microbiology, 37(5):805-812.

Hall, P. and Aller, R. (1992). Rapid, small-volume, flow injection analysis for $\sum \mathrm{CO}_{2}$ and $\mathrm{NH}_{4}{ }^{+}$in marine and fresh-waters. Limnology and Oceanography, 37(5):1113-1119.

Han, H., Hemp, J., Pace, L. A., Ouyang, H., Ganesan, K., Roh, J. H., Daldal, F., Blanke, S. R., and Gennis, R. B. (2011). Adaptation of aerobic respiration to low $\mathrm{O}_{2}$ environments. Proceedings of the National Academy of Sciences, 108(34):14109-14114.

Han, Y. and Perner, M. (2014). The Role of Hydrogen for Sulfurimonas denitrificans' Metabolism. PLoS ONE, 9(8):e106218.

Han, Y. and Perner, M. (2016). Sulfide Consumption in Sulfurimonas denitrificans and Heterologous Expression of Its Three Sulfide-Quinone Reductase Homologs. Journal of Bacteriology, 198(8):1260-1267.

Hanson, T. E., Campbell, B. J., Kalis, K. M., Campbell, M. A., and Klotz, M. G. (2013). Nitrate ammonification by Nautilia profundicola $\mathrm{AmH}$ : experimental evidence consistent 
with a free hydroxylamine intermediate. Frontiers in Evolutionary and Genomic Microbiology, 4:180.

Hedderich, R. (2004). Energy-Converting [NiFe] Hydrogenases from Archaea and Extremophiles: Ancestors of Complex I. Journal of Bioenergetics and Biomembranes, $36(1): 65-75$.

Hedderich, R., Klimmek, O., Kröger, A., Dirmeier, R., Keller, M., and Stetter, K. O. (1998). Anaerobic respiration with elemental sulfur and with disulfides. FEMS Microbiology Reviews, 22(5):353-381.

Hentscher, M. and Bach, W. (2012). Geochemically induced shifts in catabolic energy yields explain past ecological changes of diffuse vents in the East Pacific Rise $9^{\circ} 50^{\prime} \mathrm{N}$ area. Geochemical transactions, 13(1):2.

Huang, W. E., Stoecker, K., Griffiths, R., Newbold, L., Daims, H., Whiteley, A. S., and Wagner, M. (2007). Raman-FISH: combining stable-isotope Raman spectroscopy and fluorescence in situ hybridization for the single cell analysis of identity and function. Environmental Microbiology, 9(8):1878-1889.

Huber, J. A., Mark Welch, D. B., Morrison, H. G., Huse, S. M., Neal, P. R., Butterfield, D. A., and Sogin, M. L. (2007). Microbial Population Structures in the Deep Marine Biosphere. Science, 318(5847):97 -100. Cited by 0470 .

Hügler, M. and Sievert, S. M. (2011). Beyond the Calvin Cycle: Autotrophic Carbon Fixation in the Ocean. Annual Review of Marine Science, 3(1):261-289. Cited by 0055.

Hügler, M., Wirsen, C. O., Fuchs, G., Taylor, C. D., and Sievert, S. M. (2005). Evidence for Autotrophic $\mathrm{CO}_{2}$ Fixation via the Reductive Tricarboxylic Acid Cycle by Members of the $\varepsilon$ Subdivision of Proteobacteria. Journal of Bacteriology, 187(9):3020-3027. Cited by 0093.

Ikeda, T., Nakamura, M., Arai, H., Ishii, M., and Igarashi, Y. (2009). Ferredoxin-NADP+ reductase from the thermophilic hydrogen-oxidizing bacterium, Hydrogenobacter thermophilus TK-6. FEMS Microbiology Letters, 297(1):124-130. Cited by 0004.

Imlay, J. A. (2006). Iron-sulphur clusters and the problem with oxygen. Molecular Microbiology, 59(4):1073-1082.

Inagaki, F., Takai, K., Kobayashi, H., Nealson, K. H., and Horikoshi, K. (2003). Sulfurimonas autotrophica gen. nov., sp. nov., a novel sulfur-oxidizing $\varepsilon$-proteobacterium isolated from hydrothermal sediments in the Mid-Okinawa Trough. International Journal of Systematic and Evolutionary Microbiology, 53(6):1801-1805.

Inagaki, F., Takai, K., Nealson, K. H., and Horikoshi, K. (2004). Sulfurovum lithotrophicum gen. nov., sp. nov., a novel sulfur-oxidizing chemolithoautotroph within the $\varepsilon$ Proteobacteria isolated from Okinawa Trough hydrothermal sediments. International Journal of Systematic and Evolutionary Microbiology, 54(5):1477-1482.

Jannasch, H. W. (1983). Microbial Processes at Deep Sea Hydrothermal Vents. In Rona, P. A., Boström, K., Laubier, L., and Jr, K. L. S., editors, Hydrothermal Processes at Seafloor Spreading Centers, number 12 in NATO Conference Series, pages 677-709. Springer US. DOI: 10.1007/978-1-4899-0402-7_28. 
Jannasch, H. W. and Mottl, M. J. (1985). Geomicrobiology of deep-sea hydrothermal vents. Science, 229(4715):717. Cited by 0412.

Jannasch, H. W., Wirsen, C., Nelson, D., and Robertson, L. (1985). Thiomicrospira crunogena sp. nov., a colorless, sulfur-oxidizing bacterium from a deep-sea hydrothermal vent. International journal of systematic bacteriology, 35(4):422-424.

Kameya, M., Ikeda, T., Nakamura, M., Arai, H., Ishii, M., and Igarashi, Y. (2007). A Novel Ferredoxin-Dependent Glutamate Synthase from the Hydrogen-Oxidizing Chemoautotrophic Bacterium Hydrogenobacter thermophilus TK-6. Journal of Bacteriology, 189(7):2805-2812.

Kappler, U., Friedrich, C. G., Trüper, H. G., and Dahl, C. (2001). Evidence for two pathways of thiosulfate oxidation in Starkeya novella (formerly Thiobacillus novellus). Archives of Microbiology, 175(2):102-111.

Karl, D. M., Wirsen, C. O., and Jannasch, H. W. (1980). Deep-Sea Primary Production at the Galapagos Hydrothermal Vents. Science, 207(4437):1345-1347.

Kartal, B., van Niftrik, L., Keltjens, J. T., Op den Camp, H. J., and Jetten, M. S. (2012). AnammoxGrowth Physiology, Cell Biology, and Metabolism. In Advances in Microbial Physiology, volume 60, pages 211-262. Elsevier.

Kendall, J. J., Barrero-Tobon, A. M., Hendrixson, D. R., and Kelly, D. J. (2014). Hemerythrins in the microaerophilic bacterium Campylobacter jejuni help protect key ironsulphur cluster enzymes from oxidative damage. Environmental Microbiology, 16(4):1105-1121.

Kern, M. and Simon, J. (2008). Characterization of the NapGH quinol dehydrogenase complex involved in Wolinella succinogenes nitrate respiration. Molecular Microbiology, 69(5):1137-1152. 00021.

Klatt, J. M. and Polerecky, L. (2015). Assessment of the stoichiometry and efficiency of $\mathrm{CO}_{2}$ fixation coupled to reduced sulfur oxidation. Aquatic Microbiology, 6:484.

Klein, D. A. (2015). QIIME: Better Described as EMSAP? Microbe Magazine, 10(3):90-91.

Kröger, A., Biel, S., Simon, J., Gross, R., Unden, G., and Lancaster, C. R. D. (2002). Fumarate respiration of Wolinella succinogenes: enzymology, energetics and coupling mechanism. Biochimica et Biophysica Acta (BBA) - Bioenergetics, 1553(12):23-38.

Lampitt, R. S. and Antia, A. N. (1997). Particle flux in deep seas: regional characteristics and temporal variability. Deep Sea Research Part I: Oceanographic Research Papers, 44(8):1377-1403.

Lavelle, J. W. and Wetzler, M. A. (1999). Diffuse venting and background contributions to chemical anomalies in a neutrally buoyant ocean hydrothermal plume. Journal of Geophysical Research: Oceans, 104(C2):3201-3209.

Lee, S. and Fuhrman, J. A. (1987). Relationships between Biovolume and Biomass of Naturally Derived Marine Bacterioplankton. Applied and Environmental Microbiology, 53(6):1298-1303. 
Lever, M. A., Torti, A., Eickenbusch, P., Michaud, A. B., antl Temkiv, T., and Jørgensen, B. B. (2015). A modular method for the extraction of DNA and RNA, and the separation of DNA pools from diverse environmental sample types. Extreme Microbiology, 6:476.

Lin, S. and Cronan, J. E. (2011). Closing in on complete pathways of biotin biosynthesis. Molecular BioSystems, 7(6):1811-1821.

Lin, X., Wakeham, S. G., Putnam, I. F., Astor, Y. M., Scranton, M. I., Chistoserdov, A. Y., and Taylor, G. T. (2006). Comparison of Vertical Distributions of Prokaryotic Assemblages in the Anoxic Cariaco Basin and Black Sea by Use of Fluorescence In Situ Hybridization. Applied and Environmental Microbiology, 72(4):2679-2690. 00098 PMID: 16597973.

Loferer-KröSSbacher, M., Klima, J., and Psenner, R. (1998). Determination of Bacterial Cell Dry Mass by Transmission Electron Microscopy and Densitometric Image Analysis. Applied and Environmental Microbiology, 64(2):688-694.

Lowell, R., Farough, A., Germanovich, L., Hebert, L., and Horne, R. (2012). A Vent-FieldScale Model of the East Pacific Rise $9^{\circ} 50$ 'N Magma-Hydrothermal System. Oceanography, 25(1):158-167.

Luther, G. W., Findlay, A. J., MacDonald, D. J., Owings, S. M., Hanson, T. E., Beinart, R. A., and Girguis, P. R. (2011). Thermodynamics and Kinetics of Sulfide Oxidation by Oxygen: A Look at Inorganically Controlled Reactions and Biologically Mediated Processes in the Environment. Frontiers in Microbiology, 2. 00017.

Lücker, S., Nowka, B., Rattei, T., Spieck, E., and Daims, H. (2013). The genome of Nitrospina gracilis illuminates the metabolism and evolution of the major marine nitrite oxidizer. Evolutionary and Genomic Microbiology, 4:27.

Macalady, J. L., Dattagupta, S., Schaperdoth, I., Jones, D. S., Druschel, G. K., and Eastman, D. (2008). Niche differentiation among sulfur-oxidizing bacterial populations in cave waters. The ISME Journal, 2(6):590-601.

Mandernack, K. W. and Tebo, B. M. (1999). In situ sulfide removal and $\mathrm{CO}_{2}$ fixation rates at deep-sea hydrothermal vents and the oxic/anoxic interface in Framvaren Fjord, Norway. Marine Chemistry, 66(34):201-213.

Manz, W., Amann, R., Ludwig, W., Wagner, M., and Schleifer, K.-H. (1992). Phylogenetic Oligodeoxynucleotide Probes for the Major Subclasses of Proteobacteria: Problems and Solutions. Systematic and Applied Microbiology, 15(4):593-600.

Marcia, M., Ermler, U., Peng, G., and Michel, H. (2009). The structure of Aquifex aeolicus sulfide:quinone oxidoreductase, a basis to understand sulfide detoxification and respiration. Proceedings of the National Academy of Sciences, 106(24):9625-9630.

Marshall, I. P. G., Blainey, P. C., Spormann, A. M., and Quake, S. R. (2012). A single-cell genome for Thiovulum sp. Applied and Environmental Microbiology.

Marzocchi, U., Trojan, D., Larsen, S., Louise Meyer, R., Peter Revsbech, N., Schramm, A., Peter Nielsen, L., and Risgaard-Petersen, N. (2014). Electric coupling between distant nitrate reduction and sulfide oxidation in marine sediment. The ISME Journal. 
Maurice, M. S., Cremades, N., Croxen, M. A., Sisson, G., Sancho, J., and Hoffman, P. S. (2007). Flavodoxin:Quinone Reductase (FqrB): a Redox Partner of Pyruvate:Ferredoxin Oxidoreductase That Reversibly Couples Pyruvate Oxidation to NADPH Production in Helicobacter pylori and Campylobacter jejuni. Journal of Bacteriology, 189(13):4764-4773.

McCollom, T. M. and Shock, E. L. (1997). Geochemical constraints on chemolithoautotrophic metabolism by microorganisms in seafloor hydrothermal systems. Geochimica et Cosmochimica Acta, 61(20):4375-4391.

McNichol, J., Sylva, S. P., Thomas, F., Taylor, C. D., Sievert, S. M., and Seewald, J. S. (2016). Assessing Microbial Processes in Deep-Sea Hydrothermal Systems by Incubation at In Situ Temperature and Pressure. Deep Sea Research Part I: Oceanographic Research Papers.

Meier, D., Bach, W., Girguis, P. R., Gruber-Vodicka, H., Reeves, E. P., Richter, M., Vidoudez, C., Amann, R., and Meyerdierks, A. (2016). Heterotrophic Proteobacteria in the vicinity of diffuse hydrothermal venting. Environmental Microbiology, pages n/a-n/a.

Meyer, J. L. and Huber, J. A. (2014). Strain-level genomic variation in natural populations of Lebetimonas from an erupting deep-sea volcano. The ISME Journal, 8(4):867-880.

Mino, S., Kudo, H., Takayuki, A., Sawabe, T., Takai, K., and Nakagawa, S. (2014). Sulfurovum aggregans sp. nov., a novel hydrogen-oxidizing, thiosulfate-reducing chemolithoautotroph within the Epsilonproteobacteria isolated from a deep-sea hydrothermal vent chimney at the Central Indian Ridge, and an emended description of the genus Sulfurovum. International Journal of Systematic and Evolutionary Microbiology, pages ijs.0.065094-0.

Miroshnichenko, M. L., L'Haridon, S., Schumann, P., Spring, S., Bonch-Osmolovskaya, E. A., Jeanthon, C., and Stackebrandt, E. (2004). Caminibacter profundus sp. nov., a novel thermophile of Nautiliales ord. nov. within the class 'Epsilonproteobacteria', isolated from a deep-sea hydrothermal vent. International Journal of Systematic and Evolutionary Microbiology, 54(1):41-45.

Moraru, C., Lam, P., Fuchs, B. M., Kuypers, M. M. M., and Amann, R. (2010). GeneFISH an in situ technique for linking gene presence and cell identity in environmental microorganisms. Environmental Microbiology, 12(11):3057-3073.

Morris, R. L. and Schmidt, T. M. (2013). Shallow breathing: bacterial life at low $\mathrm{O}_{2}$. Nature Reviews Microbiology, 11(3):205-212.

Moussard, H., Corre, E., Cambon-Bonavita, M.-A., Fouquet, Y., and Jeanthon, C. (2006). Novel uncultured Epsilonproteobacteria dominate a filamentous sulphur mat from the $13^{\circ} \mathrm{N}$ hydrothermal vent field, East Pacific Rise. FEMS Microbiology Ecology, 58(3):449-463. 00027.

Murali, R., Yildiz, G. G., Daldal, F., and Gennis, R. B. (2012). Stoichiometry of proton

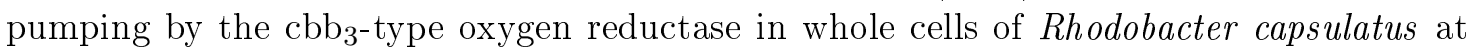
$\mathrm{pH} 7$ is about $0.5 \mathrm{H}+$ per electron. Proceedings of the National Academy of Sciences, 109(32):E2144-E2144. 
Murphy, M. J. and Siegel, L. M. (1973). Siroheme and Sirohydrochlorin the basis for a new type of porphyrin-related prosthetic group common to both assimilatory and dissimilatory sulfite reductases. Journal of Biological Chemistry, 248(19):6911-6919.

Musat, N., Foster, R., Vagner, T., Adam, B., and Kuypers, M. M. M. (2012). Detecting metabolic activities in single cells, with emphasis on nanoSIMS. FEMS Microbiology Reviews, 36(2):486-511. Cited by 0031 .

Musat, N., Halm, H., Winterholler, B., Hoppe, P., Peduzzi, S., Hillion, F., Horreard, F., Amann, R., JU00F8rgensen, B., and Kuypers, M. (2008). A single-cell view on the ecophysiology of anaerobic phototrophic bacteria. Proceedings of the National Academy of Sciences, 105(46):17861. Cited by 0140 .

Musat, N., Stryhanyuk, H., Bombach, P., Adrian, L., Audinot, J.-N., and Richnow, H. H. (2014). The effect of FISH and CARD-FISH on the isotopic composition of ${ }^{13} \mathrm{c}$ - and ${ }^{15}$ n-labeled Pseudomonas putida cells measured by nanoSIMS. Systematic and Applied Microbiology, 37(4):267-276.

Muyzer, G., Teske, A., Wirsen, C. O., and Jannasch, H. W. (1995). Phylogenetic relationships of Thiomicrospira species and their identification in deep-sea hydrothermal vent samples by denaturing gradient gel electrophoresis of $16 \mathrm{~s}$ rDNA fragments. Archives of Microbiology, 164(3):165-172.

Müller, F. H., Bandeiras, T. M., Urich, T., Teixeira, M., Gomes, C. M., and Kletzin, A. (2004). Coupling of the pathway of sulphur oxidation to dioxygen reduction: characterization of a novel membrane-bound thiosulphate:quinone oxidoreductase. Molecular Microbiology, 53(4):1147-1160.

Nakagawa, S., Inagaki, F., Takai, K., Horikoshi, K., and Sako, Y. (2005a). Thioreductor micantisoli gen. nov., sp. nov., a novel mesophilic, sulfur-reducing chemolithoautotroph within the $\varepsilon$-Proteobacteria isolated from hydrothermal sediments in the MidOkinawa Trough. International Journal of Systematic and Evolutionary Microbiology, 55(2):599-605.

Nakagawa, S. and Takai, K. (2008). Deep-sea vent chemoautotrophs: diversity, biochemistry and ecological significance. FEMS microbiology ecology, 65(1):1-14.

Nakagawa, S., Takai, K., Inagaki, F., Hirayama, H., Nunoura, T., Horikoshi, K., and Sako, Y. (2005b). Distribution, phylogenetic diversity and physiological characteristics of $\varepsilon$-Proteobacteria in a deep-sea hydrothermal field. Environmental Microbiology, $7(10): 1619-1632$.

Nakagawa, S., Takai, K., Inagaki, F., Horikoshi, K., and Sako, Y. (2005c). Nitratiruptor tergarcus gen. nov., sp. nov. and Nitratifractor salsuginis gen. nov., sp. nov., nitrate-reducing chemolithoautotrophs of the $\varepsilon$-Proteobacteria isolated from a deep-sea hydrothermal system in the Mid-Okinawa Trough. International Journal of Systematic and Evolutionary Microbiology, 55(2):925-933.

Nakagawa, S., Takaki, Y., Shimamura, S., Reysenbach, A.-L., Takai, K., and Horikoshi, K. (2007). Deep-sea vent $\varepsilon$-proteobacterial genomes provide insights into emergence of pathogens. Proceedings of the National Academy of Sciences, 104(29):12146-12150. 
Nakamura, K. and Takai, K. (2015). Geochemical Constraints on Potential Biomass Sustained by Subseafloor WaterRock Interactions. In Subseafloor Biosphere Linked to Hydrothermal Systems, pages 11-30. Springer.

Nicholls, D. G. and Ferguson, S. J. (2013). Respiratory Chains. In Bioenergetics, pages 91-157. Elsevier.

Oh, J. K. and Suzuki, I. (1977a). Isolation and Characterization of a Membrane-associated Thiosulphate-oxidizing System of Thiobacillus novellus. Journal of General Microbiology, 99(2):397-412.

Oh, J. K. and Suzuki, I. (1977b). Resolution of a Membrane-associated Thiosulphateoxidizing Complex of Thiobacillus novellus. Journal of General Microbiology, 99(2):413-423.

Orphan, V. J., House, C. H., Hinrichs, K.-U., McKeegan, K. D., and DeLong, E. F. (2001). Methane-Consuming Archaea Revealed by Directly Coupled Isotopic and Phylogenetic Analysis. Science, 293(5529):484-487. Cited by 0621.

Orth, J. D., Thiele, I., and Palsson, B. (2010). What is flux balance analysis? Nature Biotechnology, 28(3):245-248.

Ouverney, C. C. and Fuhrman, J. A. (1999). Combined Microautoradiography16s rRNA Probe Technique for Determination of Radioisotope Uptake by Specific Microbial Cell Types In Situ. Applied and Environmental Microbiology, 65(4):1746-1752.

Pace, N. R. (1997). A Molecular View of Microbial Diversity and the Biosphere. Science, 276(5313):734-740.

Pao, S. S., Paulsen, I. T., Saier, M. H., and Jr (1998). Major Facilitator Superfamily. Microbiology and Molecular Biology Reviews, 62(1):1.

Perez-Rodriguez, I., Ricci, J., Voordeckers, J. W., Starovoytov, V., and Vetriani, C. (2010). Nautilia nitratireducens sp. nov., a thermophilic, anaerobic, chemosynthetic, nitrateammonifying bacterium isolated from a deep-sea hydrothermal vent. International Journal of Systematic and Evolutionary Microbiology, 60(5):1182-1186.

Perner, M., Hansen, M., Seifert, R., Strauss, H., Koschinsky, A., and Petersen, S. (2013). Linking geology, fluid chemistry, and microbial activity of basalt- and ultramafic-hosted deep-sea hydrothermal vent environments. Geobiology, 11(4):340-355. 00001.

Perner, M., Hentscher, M., Rychlik, N., Seifert, R., Strauss, H., and Bach, W. (2011). Driving forces behind the biotope structures in two low-temperature hydrothermal venting sites on the southern Mid-Atlantic Ridge. Environmental Microbiology Reports, 3(6):727-737.

Perner, M., Petersen, J. M., Zielinski, F., Gennerich, H.-H., and Seifert, R. (2010). Geochemical constraints on the diversity and activity of $\mathrm{H}_{2}$-oxidizing microorganisms in diffuse hydrothermal fluids from a basalt- and an ultramafic-hosted vent. FEMS Microbiology Ecology, 74(1):55-71.

Pernthaler, A., Pernthaler, J., and Amann, R. (2002). Fluorescence In Situ Hybridization and Catalyzed Reporter Deposition for the Identification of Marine Bacteria. Applied and Environmental Microbiology, 68(6):3094 -3101. 
Polerecky, L., Adam, B., Milucka, J., Musat, N., Vagner, T., and Kuypers, M. M. M. (2012). Look@NanoSIMS - a tool for the analysis of nanoSIMS data in environmental microbiology. Environmental Microbiology, 14(4):1009-1023.

Polz, M. F. and Cavanaugh, C. M. (1995). Dominance of one bacterial phylotype at a MidAtlantic Ridge hydrothermal vent site. Proceedings of the National Academy of Sciences of the United States of America, 92(16):7232.

Ponsard, J., Cambon-Bonavita, M.-A., Zbinden, M., Lepoint, G., Joassin, A., Corbari, L., Shillito, B., Durand, L., Cueff-Gauchard, V., and Compère, P. (2013). Inorganic carbon fixation by chemosynthetic ectosymbionts and nutritional transfers to the hydrothermal vent host-shrimp Rimicaris exoculata. The ISME Journal, 7(1):96-109.

Potter, L., Millington, P., Griffiths, L., Thomas, G., and Cole, J. (1999). Competition between Escherichia coli strains expressing either a periplasmic or a membrane-bound nitrate reductase: does Nap confer a selective advantage during nitrate-limited growth? Biochem. J, 344:77-84. 00095.

Preisig, O., Zufferey, R., Thöny-Meyer, L., Appleby, C. A., and Hennecke, H. (1996). A HighAffinity cbb3-Type Cytochrome Oxidase Terminates the Symbiosis-Specific Respiratory Chain of Bradyrhizobium japonicum. Journal of Bacteriology, 178(6):1532-1538.

Proskurowski, G., Lilley, M. D., and Olson, E. J. (2008). Stable isotopic evidence in support of active microbial methane cycling in low-temperature diffuse flow vents at $9^{\circ} 50^{\prime} \mathrm{N}$ East Pacific Rise. Geochimica et Cosmochimica Acta, 72(8):2005-2023.

Quast, C., Pruesse, E., Yilmaz, P., Gerken, J., Schweer, T., Yarza, P., Peplies, J., and Glöckner, F. O. (2013). The SILVA ribosomal RNA gene database project: improved data processing and web-based tools. Nucleic Acids Research, 41(D1):D590-D596.

Rauhamäki, V., Bloch, D. A., and Wikström, M. (2012a). Mechanistic stoichiometry of proton translocation by cytochrome $\mathrm{cbb}_{3}$. Proceedings of the National Academy of Sciences, 109(19):7286-7291.

Rauhamäki, V., Bloch, D. A., and Wikström, M. (2012b). Reply to Murali et al.: Proton translocation stoichiometry of $\mathrm{cbb}_{3}$-type cytochrome c oxidase. Proceedings of the National Academy of Sciences, 109(32):E2145-E2145.

Rauhamäki, V. and Wikström, M. (2014). The causes of reduced proton-pumping efficiency in type $\mathrm{B}$ and $\mathrm{C}$ respiratory heme-copper oxidases, and in some mutated variants of type A. Biochimica et Biophysica Acta (BBA) - Bioenergetics, 1837(7):999-1003.

Rawlings, N. D., Barrett, A. J., and Bateman, A. (2010). MEROPS: the peptidase database. Nucleic Acids Research, 38(suppl 1):D227-D233.

Reeves, E. P., McDermott, J. M., and Seewald, J. S. (2014). The origin of methanethiol in midocean ridge hydrothermal fluids. Proceedings of the National Academy of Sciences, 111(15):5474-5479.

Seewald, J. S., Doherty, K. W., Hammar, T. R., and Liberatore, S. P. (2002). A new gastight isobaric sampler for hydrothermal fluids. Deep Sea Research Part I: Oceanographic Research Papers, 49(1):189-196. Cited by 0047. 
Sherr, B., Sherr, E., and del Giorgio, P. (2001). Enumeration of total and highly active bacteria. In John H Paul, editor, Methods in Microbiology, volume Volume 30, pages 129-159. Academic Press. Cited by 0056.

Sievert, S. M., Scott, K. M., Klotz, M. G., Chain, P. S. G., Hauser, L. J., Hemp, J., Hugler, M., Land, M., Lapidus, A., Larimer, F. W., Lucas, S., Malfatti, S. A., Meyer, F., Paulsen, I. T., Ren, Q., Simon, J., and the USF Genomics Class (2008). Genome of the Epsilonproteobacterial Chemolithoautotroph Sulfurimonas denitrificans. Applied and Environmental Microbiology, 74(4):1145-1156. Cited by 0000.

Sievert, S. M. and Vetriani, C. (2012). Chemoautotrophy at deep-sea vents: Past, present, and future. Oceanography, 25(1):218-233.

Sievert, S. M., Wieringa, E. B. A., Wirsen, C. O., and Taylor, C. D. (2007). Growth and mechanism of filamentous-sulfur formation by Candidatus Arcobacter sulfidicus in opposing oxygen-sulfide gradients. Environmental Microbiology, 9(1):271-276. Cited by 0027 .

Signori, C. N., Thomas, F., Enrich-Prast, A., Pollery, R. C. G., and Sievert, S. M. (2014). Microbial diversity and community structure across environmental gradients in Bransfield Strait, Western Antarctic Peninsula. Frontiers in Microbiology, 5.

Sikorski, J., Munk, C., Lapidus, A., Djao, O. D. N., Lucas, S., Del Rio, T. G., Nolan, M., Tice, H., Han, C., and Cheng, J.-F. (2010). Complete genome sequence of Sulfurimonas autotrophica type strain (OK10t). Standards in genomic sciences, 3(2):194.

Simon, J., van Spanning, R. J., and Richardson, D. J. (2008). The organisation of proton motive and non-proton motive redox loops in prokaryotic respiratory systems. Biochimica et Biophysica Acta (BBA) - Bioenergetics, 1777(12):1480-1490.

Sonnhammer, E. L., von Heijne, G., and Krogh, A. (1998). A hidden Markov model for predicting transmembrane helices in protein sequences. In Ismb, volume 6, pages 175-182.

Spencer, J. B., Stolowich, N. J., Roessner, C. A., and Scott, A. (1993). The Escherichia coli cysG gene encodes the multifunctional protein, siroheme synthase. FEBS Letters, $335(1): 57-60$.

Stackebrandt, E., Murray, R. G. E., and Trüper, H. G. (1988). Proteobacteria classis nov., a Name for the Phylogenetic Taxon That Includes the Purple Bacteria and Their Relatives. International Journal of Systematic and Evolutionary Microbiology, 38(3):321-325.

Steffensen, J. L., Dufault-Thompson, K., and Zhang, Y. (2016). PSAMM: A Portable System for the Analysis of Metabolic Models. PLoS Comput Biol, 12(2):e1004732.

Stokke, R., Dahle, H., Roalkvam, I., Wissuwa, J., Daae, F. L., Tooming-Klunderud, A., Thorseth, I. H., Pedersen, R. B., and Steen, I. H. (2015). Functional interactions among filamentous Epsilonproteobacteria and Bacteroidetes in a deep-sea hydrothermal vent biofilm. Environmental Microbiology, 17(10):4063-4077.

Taboada, B., Verde, C., and Merino, E. (2010). High accuracy operon prediction method based on STRING database scores. Nucleic Acids Research, page gkq254. 00013 PMID: 20385580 . 
Takai, K., Campbell, B. J., Cary, S. C., Suzuki, M., Oida, H., Nunoura, T., Hirayama, H., Nakagawa, S., Suzuki, Y., Inagaki, F., and Horikoshi, K. (2005). Enzymatic and Genetic Characterization of Carbon and Energy Metabolisms by Deep-Sea Hydrothermal Chemolithoautotrophic Isolates of Epsilonproteobacteria. Applied and Environmental Microbiology, 71(11):7310-7320. Cited by 0080.

Takai, K., Inagaki, F., Nakagawa, S., Hirayama, H., Nunoura, T., Sako, Y., Nealson, K. H., and Horikoshi, K. (2003). Isolation and phylogenetic diversity of members of previously uncultivated $\varepsilon$-Proteobacteria in deep-sea hydrothermal fields. FEMS Microbiology Letters, 218(1):167-174.

Takai, K., Nealson, K. H., and Horikoshi, K. (2004). Hydrogenimonas thermophila gen. nov., sp. nov., a novel thermophilic, hydrogen-oxidizing chemolithoautotroph within the $\varepsilon$-Proteobacteria, isolated from a black smoker in a Central Indian Ridge hydrothermal field. International Journal of Systematic and Evolutionary Microbiology, 54(1):25-32. Cited by 0052 .

Takai, K., Suzuki, M., Nakagawa, S., Miyazaki, M., Suzuki, Y., Inagaki, F., and Horikoshi, K. (2006). Sulfurimonas paralvinellae Sp. Nov., a Novel Mesophilic, Hydrogen- and Sulfur-Oxidizing Chemolithoautotroph Within the Epsilonproteobacteria Isolated from a Deep-Sea Hydrothermal Vent Polychaete Nest, Reclassification of Thiomicrospira denitrificans as Sulfurimonas denitrificans Comb. Nov. and Emended Description of the Genus Sulfurimonas. International Journal of Systematic and Evolutionary Microbiology, 56(8):1725-1733.

Thiele, I., Vo, T. D., Price, N. D., and Palsson, B. (2005). Expanded metabolic reconstruction of Helicobacter pylori (iIT341 GSM/GPR): an in silico genome-scale characterization of single-and double-deletion mutants. Journal of Bacteriology, 187(16):5818-5830.

Thompson, A. W., Foster, R. A., Krupke, A., Carter, B. J., Musat, N., Vaulot, D., Kuypers, M. M. M., and Zehr, J. P. (2012). Unicellular Cyanobacterium Symbiotic with a SingleCelled Eukaryotic Alga. Science, 337(6101):1546-1550.

Timmer-Ten Hoor, A. (1975). A new type of thiosulphate oxidizing, nitrate reducing microorganism: Thiomicrospira denitrificans sp. Nov. Netherlands Journal of Sea Research, $9(34): 344-350$.

Timmer-Ten Hoor, A. (1981). Cell yield and bioenergetics of Thiomicrospira denitrificans compared with Thiobacillus denitrificans. Antonie van Leeuwenhoek, 47(3):231-243.

Tivey, M. K. (2007). Generation of seafloor hydrothermal vent fluids and associated mineral deposits. Oceanography.

Tomb, J.-F., White, O., Kerlavage, A. R., Clayton, R. A., Sutton, G. G., Fleischmann, R. D., Ketchum, K. A., Klenk, H. P., Gill, S., Dougherty, B. A., Nelson, K., Quackenbush, J., Zhou, L., Kirkness, E. F., Peterson, S., Loftus, B., Richardson, D., Dodson, R., Khalak, H. G., Glodek, A., McKenney, K., Fitzegerald, L. M., Lee, N., Adams, M. D., Hickey, E. K., Berg, D. E., Gocayne, J. D., Utterback, T. R., Peterson, J. D., Kelley, J. M., Cotton, M. D., Weidman, J. M., Fujii, C., Bowman, C., Watthey, L., Wallin, E., Hayes, W. S., Borodovsky, M., Karp, P. D., Smith, H. O., Fraser, C. M., and Venter, J. C. (1997). The complete genome sequence of the gastric pathogen Helicobacter pylori. Nature, $388(6642): 539-547$. 
Tremblay, P.-L., Zhang, T., Dar, S. A., Leang, C., and Lovley, D. R. (2013). The Rnf Complex of Clostridium ljungdahlii Is a Proton-Translocating Ferredoxin:NAD+ Oxidoreductase Essential for Autotrophic Growth. mBio, 4(1):e00406-12. Cited by 0003.

Tuttle, J. H., Wirsen, C. O., and Jannasch, H. W. (1983). Microbial activities in the emitted hydrothermal waters of the Galapagos Rift vents. Marine Biology, 73(3):293-299.

Urich, T., Lanzén, A., Stokke, R., Pedersen, R. B., Bayer, C., Thorseth, I. H., Schleper, C., Steen, I. H., and Øvreas, L. (2014). Microbial community structure and functioning in marine sediments associated with diffuse hydrothermal venting assessed by integrated meta-omics. Environmental Microbiology, 16(9):2699-2710.

Veldkamp, H. and Jannasch, H. W. (1972). Mixed culture studies with the chemostat. Journal of Applied Chemistry and Biotechnology, 22(1):105-123.

Vetriani, C., Voordeckers, J. W., Crespo-Medina, M., O’Brien, C. E., Giovannelli, D., and Lutz, R. A. (2014). Deep-sea hydrothermal vent Epsilonproteobacteria encode a conserved and widespread nitrate reduction pathway (Nap). The ISME Journal. Cited by 0000.

Von Damm, K. L. and Lilley, M. D. (2004). Diffuse Flow Hydrothermal Fluids from $9^{\circ}$ 50' N East Pacific Rise: Origin, Evolution and Biogeochemical Controls. In Wilcock, W. S. D., Delong, E. F., Kelley, D. S., Baross, J. A., and Cary, S. C., editors, The Subseafloor Biosphere at Mid-Ocean Ridges, pages 245-268. American Geophysical Union.

Wankel, S. D., Germanovich, L. N., Lilley, M. D., Genc, G., DiPerna, C. J., Bradley, A. S., Olson, E. J., and Girguis, P. R. (2011). Influence of subsurface biosphere on geochemical fluxes from diffuse hydrothermal fluids. Nature Geoscience, 4(7):461-468.

Weerakoon, D. R. and Olson, J. W. (2008). The Campylobacter jejuni NADH:Ubiquinone Oxidoreductase (Complex I) Utilizes Flavodoxin Rather than NADH. Journal of Bacteriology, 190(3):915-925.

Whiteley, A. S., Manefield, M., and Lueders, T. (2006). Unlocking the 'microbial black box'using RNA-based stable isotope probing technologies. Current opinion in biotechnology, 17(1):67-71.

Winkel, M., Pjevac, P., Kleiner, M., Littmann, S., Meyerdierks, A., Amann, R., and MuSSmann, M. (2014). Identification and activity of acetate-assimilating bacteria in diffuse fluids venting from two deep-sea hydrothermal systems. FEMS Microbiology Ecology, 90(3):731-746.

Wirsen, C. O. and Jannasch, H. W. (1978). Physiological and morphological observations on Thiovulum sp. Journal of Bacteriology, 136(2):765.

Wirsen, C. O., Jannasch, H. W., and Molyneaux, S. J. (1993). Chemosynthetic microbial activity at Mid-Atlantic Ridge hydrothermal vent sites. Journal of geophysical research, 98(B6):9693-9703. Cited by 0094.

Wirsen, C. O., Sievert, S. M., Cavanaugh, C. M., Molyneaux, S. J., Ahmad, A., Taylor, L. T., DeLong, E. F., and Taylor, C. D. (2002). Characterization of an Autotrophic Sulfide-Oxidizing Marine Arcobacter sp. That Produces Filamentous Sulfur. Appl. Environ. Microbiol., 68(1):316-325. Cited by 0156. 
Wirsen, C. O., Tuttle, J. H., and Jannasch, H. W. (1986). Activities of sulfur-oxidizing bacteria at the $21 \mathrm{~N}$ East Pacific Rise vent site. Marine Biology, 92(4):449-456.

Woese, C. R. and Fox, G. E. (1977). Phylogenetic structure of the prokaryotic domain: The primary kingdoms. Proceedings of the National Academy of Sciences, 74(11):5088-5090.

Wolin, M. J., Wolin, E. A., and Jacobs, N. J. (1961). Cytochrome-producing anaerobic vibrio, Vibrio succinogenes, sp. n. Journal of Bacteriology, 81(6):911.

Yamamoto, M., Nakagawa, S., Shimamura, S., Takai, K., and Horikoshi, K. (2010). Molecular characterization of inorganic sulfur-compound metabolism in the deep-sea epsilonproteobacterium Sulfurovum sp. NBC37-1. Environmental Microbiology, 12(5):1144-1153.

Yamamoto, M. and Takai, K. (2011). Sulfur Metabolisms in Epsilon- and Gammaproteobacteria in Deep-Sea Hydrothermal Fields. Frontiers in Microbiology, 2.

Zeikus, J. G. (1979). Thermophilic bacteria: Ecology, physiology and technology. Enzyme and Microbial Technology, 1(4):243-252.

Zhang, Y. and Sievert, S. M. (2014). Pan-genome analyses identify lineage- and niche-specific markers of evolution and adaptation in Epsilonproteobacteria. Frontiers in Microbiology, 5.

Zhu, C. J. and Lee, Y. K. (1997). Determination of biomass dry weight of marine microalgae. Journal of Applied Phycology, 9(2):189-194. 00108.

Zumft, W. G. (1997). Cell biology and molecular basis of denitrification. Microbiology and Molecular Biology Reviews, 61(4):533-616. 